\title{
PRECISION MEASUREMENT OF THE PROTON MAGNETIC FORM FACTOR AT HIGH $Q^{2}$
}

\author{
A Dissertation \\ by \\ THIR NARAYAN SHARMA GAUTAM
}

Submitted to the Graduate College of Hampton University in partial fulfillment of the requirements for the degree of

DOCTOR OF PHILOSOPHY

MAY 2019 
This dissertation submitted by Thir Narayan Sharma Gautam in partial fulfillment of the requirements for the degree of Doctor of Philosophy at Hampton University, Hampton, Virginia is hereby approved by the committee under whom the work has been completed.

M. Eric Christy, Ph.D.

Committee Chair

Michael Kohl, Ph.D.

José L. Goity , Ph.D.

David J. Mack, Ph.D.

Michelle Penn-Marshall, Ph.D.

Dean, The Graduate College

Date 


\begin{abstract}
Precision Measurement of the Proton Magnetic Form Factor at High $Q^{2}$

Hampton University(May 2019)

Thir Narayan Sharma Gautam, M.S., The University of Southern Mississippi;

Ph.D., Hampton University

Chair of Advisory Committee: Dr. M. Eric Christy
\end{abstract}

Elastic electromagnetic form factors characterize the distribution of electric charge and magnetization current inside the nucleon and reflect the internal structure determined by Quantum Chromodynamics. Existing data on the proton magnetic form factor at high $Q^{2}$ (the 4-momentum transfer squared) have large statistical and systematic uncertainties. The GMp experiment E12-07-108 was one of the first set of experiments to run in Hall A at Jefferson Lab after the $12 \mathrm{GeV}$ upgrade with the goal of this experiment to precisely measure the electron-proton elastic cross section for $Q^{2}$ up to $17 \mathrm{GeV}^{2}$ with an accuracy of better than $2 \%$ - several times better than existing data in this $Q^{2}$ range. This will allow further tests of form factor scaling predicted by pQCD and, additionally, will be an important benchmark for many other experiments where elastic electron-proton scattering is used for normalization. Since, GMp data were taken at lower $\epsilon$ (virtual photon polarization) than SLAC data, the measured cross sections recieve a smaller contribution from the electric form factor. This dissertation analyzed the GMp experiment data for five different kinematic settings determining the form factor with total uncertainties nearly two times smaller than the existing data at these $Q^{2}$. This dissertation also presents the results of all Fall 2016 and Spring 2016 GMp experiment data. The proton magnetic form factors were extracted using both the Rosenbluth separation method and a parameterization of the form factor ratio $\left(\mu_{p} G_{E}^{p} / G_{M}^{p}\right)$ obtained from a fit to the existing cross section data. At the end of this thesis, the extracted GMp form factor data is compared to the existing data. The results for $\mu_{p} G_{E}^{p} / G_{M}^{p}$ from Rosenbluth separations are found to be consistent with previous extractions, 
but with significantly reduced uncertainties, further highlighting the discrepancy with the polarization transfer technique. This data set can provide significant additional constraints on contributions from two-photon exchange. 
Dedicated to my parents, my wife and my daughters Ishara and Pritisha. 


\section{ACKNOWLEDGEMENTS}

I want to express my appreciation to the people who assisted during my Ph.D. study. First of all, my greatest heartfelt gratitude goes to my supervisor Dr. Eric Christy who offered his endless and energetic advice, encouragement and undertook tremendous responsibility in supervising me to the completion of my research and dissertation. I thank him for the systematic guidance, patience, motivation, enthusiasm, great effort and immense knowledge he put into training me in the scientific field. I could not have imagined having a better advisor and mentor for my study.

I was so fortunate to join the GMp collaboration and closely work with Dr. Bogdan Wojtsekhowski and Dr. Shalev Gilad. I have learned a lot from Bogdan. His guidance and supervision during the experimental run not only enhanced my knowledge in nuclear physics but also improved my analysis and problem-solving abilities. I would like to thank Dr. Dave Mack for valuable suggestions and comments on the data analysis, especially in regards to the beam change measurement, and for serving as one of my committee members. I extend my gratitude to Dr. Michael Kohl and Dr. José L. Goity for their valuable time for academic development and serving on my committee.

I also would like to thank post-docs Dr. Vincent Sulkesly, Dr. Kalyan Alada, Dr. Vardan Khachatryan, Dr. Narbe Kalantarians and Dr. Luke Mayers who shared great patience in teaching me the knowledge of data analysis and earnestly answer every question.

I would like to thank my colleagues and fellow Ph.D. students on the GMp collaboration: Yang Wang, Longwu Ou, Barak Schmooker and Bashar Aljawrneh for their help and cooperation, as the experimental results of this dissertation, is based on their collaborative contributions to the calibrations of the experimental setup and data analysis. I can't forget our friends from the DVCS collaboration: Mongi Dlamini, and Bishnu Karki for their friendship and willingness to calmly entertain all my questions. I would also like to thank the friends I knew during my work at Jefferson Lab: Dr. Dien Nguyen, Shujie Li, Dr. Sheren Al- 
salmi, Jason Bane, Tong Su, Mike Nycz, Debaditya Biswas, Bishnu Panday, Bishoy Dongwi, Dr. Shankar Dayal Adhikari, Dr. Ishara Fernando, Aruni Nadeeshani and, many others. Finally, I take this opportunity to express my profound gratitude to my dear wife Sabita Sapkota, my daughters Ishara and Pritisha, my beloved parents, my brother Dr. Lekh Nath Sharma Gautam and my siblings for their love, encouragement, and continuous support during all these years. 


\section{TABLE OF CONTENTS}

Chapter

Page

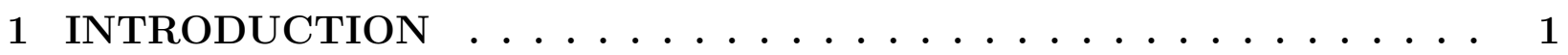

1.1 Form Factors . . . . . . . . . . . . . . . . . . . . 4

1.2 Hadronic Currents . . . . . . . . . . . . . . . . . . . . 5

1.3 Elastic Scattering . . . . . . . . . . . . . . . . . . 8

1.4 Experimental Measurement of Form Factors . . . . . . . . . . . . . . . . 12

1.5 Two-photon Exchange for Unpolarized Scattering . . . . . . . . . . . . . 14

1.6 Global Fit . . . . . . . . . . . . . . . . . . . 19

1.7 Models of Form Factors _. . . . . . . . . . . . . . . . . . . . . . 21

1.7.1 Vector Meson Dominance ... . . . . . . . . . . . . . . 21

1.7.2 Perturbative QCD (pQCD) . . . . . . . . . . . . 24

1.8 Existing Magnetic Form Factor Data . . . . . . . . . . . . . 26

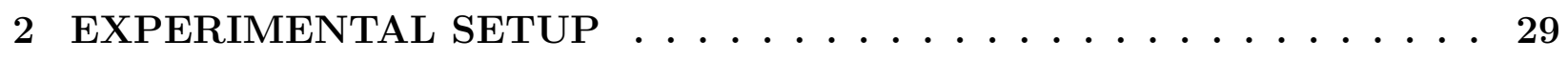

2.1 Overview . . . . . . . . . . . . . . . . . . . . . . . . . . 29

2.2 Thomas Jefferson National Accelerator Facility . . . . . . . . . . . . . . . . . 29

2.3 Hall A Overview . . . . . . . . . . . . . . . . . . . . . . . 31

2.4 Hall A Beamline Instrumentation . . . . . . . . . . . . . . . . . 31

2.4.1 The Hall A Arc and Beam Energy Measurement . . . . . . . . . . . . 31

2.4.2 Beam Position Measurement . . . . . . . . . . . . . . . 34

2.4 .3 Beam Rastering . . . . . . . . . . . . . . . . . 36

2.4.4 Beam Current Measurement . . . . . . . . . . . . . . . . 37

2.5 Scattering Chamber . . . . . . . . . . . . . . . . . 39

2.6 High Resolution Spectrometer . . . . . . . . . . . . . . . . . . . . 42 
2.7 HRS Detector Package . . . . . . . . . . . . . . . . . 43

2.8 Vertical Drift Chamber . . . . . . . . . . . . . . . . . . . 44

2.9 Coordinate Systems . . . . . . . . . . . . . . . . . . 45

2.9.1 Hall Coordinates . . . . . . . . . . . . . . . 46

2.9.2 Target Coordinates . . . . . . . . . . . . . . 46

2.9.3 Detector Coordinate System . . . . . . . . . . . . . . . 47

2.9.4 Transport Coordinate System . . . . . . . . . . . . . 48

2.9.5 Focal Plane Coordinate System . . . . . . . . . . . . . . . . 49

2.10 Straw Chamber . . . . . . . . . . . . . . . . 50

2.11 Scintillator Planes . . . . . . . . . . . . . . . . . . . 51

2.12 Gas Cherenkov . . . . . . . . . . . . . . . . 52

2.13 Lead Glass Calorimeter . . . . . . . . . . . . . . . . . 54

2.14 Data Acquisition and Trigger System . . . . . . . . . . . . . 56

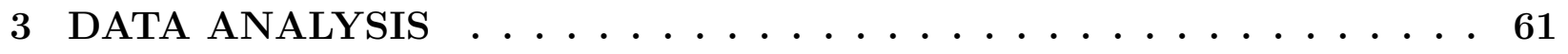

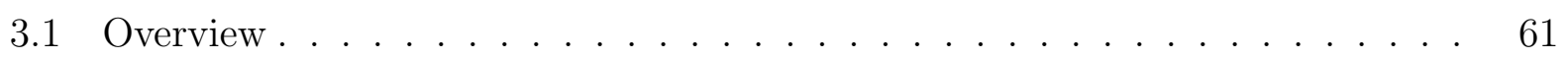

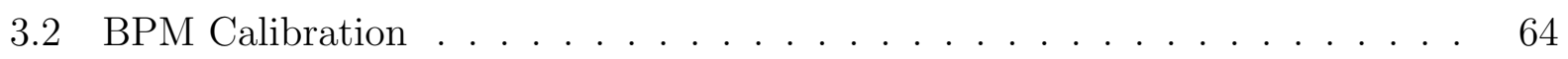

3.3 Beam Charge Calibration . . . . . . . . . . . . . . . 67

3.3.1 Unser Wire Calibration . . . . . . . . . . . . . . . 67

3.3.2 BCM Calibration ........................ 69

3.3.2.1 Stability of Gain and Offsets . . . . . . . . . . . . 72

3.3.2.2 Global BCM Calibration . . . . . . . . . . . 73

3.4 Target Boiling Study . . . . . . . . . . . . . . . . . 78

3.4.1 Selection of Good Events for Yield Analysis . . . . . . . . . . . . . 79

3.4.2 Preliminary Results of Target Boiling Study . . . . . . . . . . . . . 79

3.4.3 Offset in Measured Current . . . . . . . . . . . . . 80 
3.5 Vertical Drift Chamber Tracking Efficiency . . . . . . . . . . . . . . 88

3.6 PID Efficiencies . . . . . . . . . . . . . . . . . 93

3.6.1 Selection of Events . . . . . . . . . . . . . . . . 93

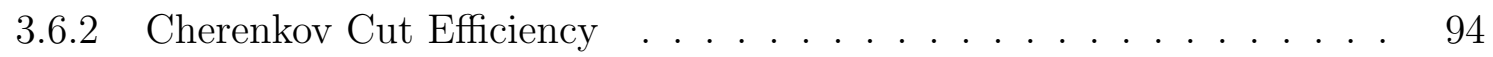

3.6.3 Calorimeter Efficiency _....................... 97

3.7 Computer and Electronic Dead Time . . . . . . . . . . . . . . . . 101

3.8 HRS Optics . . . . . . . . . . . . . . . . . . 102

3.8.1 Optics Optimization Procedure . . . . . . . . . . . 103

3.8.1.1 Target y Calibration ............... 106

3.8.1.2 Calibration of $x_{t a r}^{\prime}$ and $y_{\text {tar }}^{\prime} \ldots \ldots \ldots$. . . . . . . 107

3.8.1.3 Momentum Calibration ............... 108

3.8.2 Check of Optics Across Angular Acceptance . . . . . . . . . . . . . . 110

3.9 Determination of Target and Spectrometer Offsets . . . . . . . . . . . 111

3.10 SIMC Simulation . . . . . . . . . . . . . . . . . . 114

3.11 Radiative Correction . . . . . . . . . . . . . . . . . 115

3.12 Ionization Loss . . . . . . . . . . . . . . . . . . . . 120

3.13 HRS Optics with Saturated Q1 . . . . . . . . . . . . . 121

3.14 MC Uncertainties in Spectrometer Modeling for LHRS Fall 2016 . . . . . . . 131

3.15 Acceptance Studies: Separating Optics from Acceptance . . . . . . . . . . . 132

4 EXTRACTION OF THE ELASTIC CROSS SECTION . . . . . . 135

4.0.1 The Monte Carlo Ratio Method . . . . . . . . . . . . . . . 136

4.0.1.1 Selection of Good Electron Events . . . . . . . . . . . . . . 137

4.0.1.2 Subtraction of Background Events from Target Cell Endcaps 138

4.0.1.3 Cross Section Extraction . . . . . . . . . . . . . . . 139

4.0.1.4 Systematic Uncertainties . . . . . . . . . . . . . 141 
4.0.2 Cross Section Extraction by Acceptance Correction Method . . . . . 146 4.0.2.1 Procedure ..................... 147

4.0.2.2 Acceptance Correction . . . . . . . . . . . . . . . . 148

4.0.2.3 Bin-Centering Correction and Averaging . . . . . . . . . . . 150

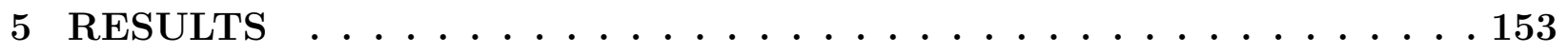

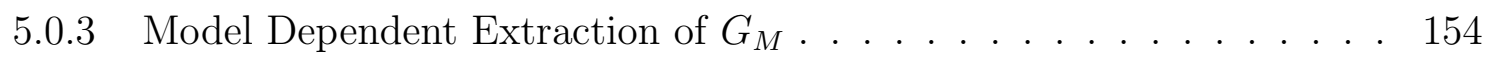

5.0 .4 Rosenbluth Separation . . . . . . . . . . . . . . 155

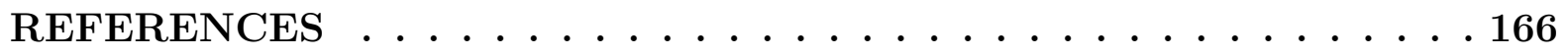

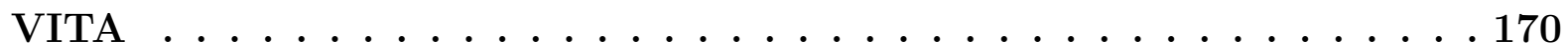




\section{LIST OF TABLES}

Table

Page

1.1

Parameters for the fit to the TPE-uncorrected cross section, using the parameterization for $F_{m}$ and $F_{e} \ldots \ldots \ldots \ldots \ldots \ldots \ldots$

2.1

The characteristics of the Hall A HRSs. . . . . . . . . . . . . . . . . . . . . . . 43

3.1

Unser wire calibration coefficient table from three different run periods. . . . . 67

Gains (in $\times 10^{-6} \mu \mathrm{A} / \mathrm{Hz}$ ) and offsets (in $\times 10^{-6} \mu \mathrm{A}$ ) for $\mathrm{u} 1$, d1 and $\mathrm{d} 3 \mathrm{BCM}$ receivers determined from different calibration runs. . . . . . . . . . . 74

3.3

Gains (in $\times 10^{-6} \mu \mathrm{A} / \mathrm{Hz}$ ) and offsets (in $\times 10^{-6} \mu \mathrm{A}$ ) for $\mathrm{d} 10$, unew and dnew $\mathrm{BCM}$ receivers determined from different calibration runs. Calibrations 1-5 were taken in Spring 2016 and 6-8 were taken in Fall 2016 . . . . . . . . . . . . 75

Comparison table of extracted VDC one-track and one-cluster efficiencies for all GMp Fall 2016 kinematics taken from [1]. One cluster efficiency was used for analysis. . . . . . . . . . . . . . . . . . . . . 90

The table of PID cut efficiencies for all Fall 2016 GMp kinematics. Table is from Ref. [1]. . . . . . . . . . . . . . . . . . . . . . . 99

The relative uncertainties on electron efficiency, pion rejection efficiency, and final pion-to-electron ratio for all Fall 2016 GMp kinematics. An uncertainty of $5 \times 10^{-4}\left(1 \times 10^{-4}\right)$ on the electron (pion) efficiency due to the sample selection and also applied an uncertainty of $1 \times 10^{-4}$ on both the electron and pion efficiency due to the E/p threshold. Table is from Ref. [1] . . . . . . . . . . . 100 
Computer live-times for all GMp Fall 2016 kinematics. The relative uncertainty were calculated assuming binomial errors. Table is taken from Ref. [1]. . . . . 101

Target $y, x$ beam position and spectrometer pointing informations for surveyed GMp Fall 2016 kinematics. . . . . . . . . . . . . . . . . . . 111

Spectrometer pointing offsets for un-surveyed LHRS Fall 2016 kinematics. . 114

Status of the Q1 field for Fall 2016 kinematics where the relative fields are shown in the last column. . . . . . . . . . . . . . . . . . . . . . . . 12

Determination of true $\int \mathrm{Bdl}$ reduction from dummy target z-vertex study. $\quad . \quad 125$

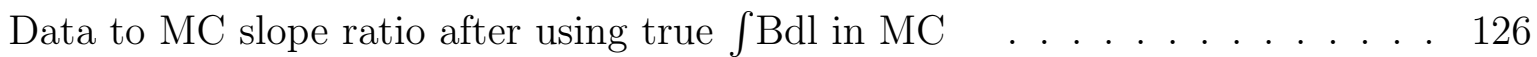

Integral comparisons for nominal, baseline and final tune.

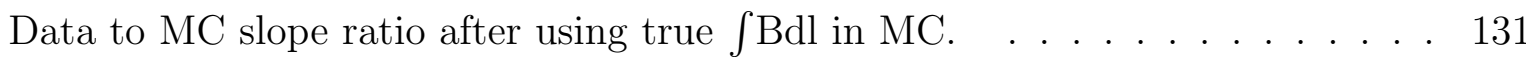

Study of MC uncertainties for kinematics K3-7.

Study of MC uncertainties by shifting the dominant apertures up and down by

$1 \mathrm{~mm}$.

Ratio of cross sections with and without software cuts on the sieve collimater.

4.1

Cuts applied to select good electron events.

Summary of point-to-point and normalization uncertainties of the Fall 2016

LHRS GMp experiment. . . . . . . . . . . . . . . . . . . 144 
4.3

Summary of the GMp Fall 2016 LHRS kinematics, extracted elastic cross sections, point-to-point and statistical uncertainties. . . . . . . . . . . . . 144

4.4

Summary of the GMp Fall 2016 RHRS kinematics, extracted elastic cross sections, point-to-point and statistical uncertainties (uncertainties are not final).

............................... 146

Tabular comparison of extracted cross section from MC ratio and acceptance correction methods. . . . . . . . . . . . . . . . . 152

5.1

Summary of magnetic from factors relative to the dipole form factor extracted utilizing the $G_{E}$ to $G_{M}$ ratio using a parametrization from the fits to the existing data. . . . . . . . . . . . . . . . . . . . 156

5.2

Summary of the Rosenbluth method form factors relative to the dipole form

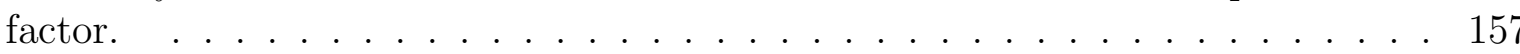




\section{LIST OF FIGURES}

Figure

Page

1.1 Ratio of the proton form factors as a function of $Q^{2}$ according to data obtained by the Rosenbluth method in (red points) and by the polarization-transfer measurements (blue points) in single photon exchange approximation. Plot is from Ref. [2]. . . . . . . . . . . . . . . . . . . . 3

1.2 Feynman diagram for electron proton elastic scattering . . . . . . . . . . 9

1.3 Schematic diagram for the recoil polarization. . . . . . . . . . . . . . . 13

1.4 Feynman diagrams for electron-proton elastic scattering: one-photon exchange (Born approximation) and two-photon exchange scattering. . . . . . . . . . .

1.5 Feynman diagrams for electron-proton elastic scattering: two-photon exchange scattering.

1.6 The ratio of proton electric and magnetic form factors measured using LT separation (red points) and polarization transfer (PT)(blue points). The LT points corrected for TPE are shown with filled square and filled circles.

1.7 A Feynman diagram for a scattering process featuring VMD in which the virtual photon couples to the nucleon (shown as a black circle) through $\rho$ or $\omega$ mesons.

1.8 Plot of $Q^{4} G_{M}^{p} / \mu_{p}$ showing the asymptotic behavior of the proton magnetic form factor. The data are: Bartel et al. is from Ref. [3], Berger et al. is from Ref. [4], Andivahis et al. is from Ref. [5], Kirk et al. Ref. [6], Sill et al. is from Ref. [7].

1.9 Proton magnetic form factor world data normalized by the dipole form factor from unpolarized measurements. The old data are from Refs. [3-7], Jlab $6 \mathrm{GeV}$ data refers to the Christy et al. Ref. [8] and JLab $12 \mathrm{GeV}$ refers to the data this experiment collected. Uncertainties shown do not include the effect of the normalization uncertainty, which is $3 \%$ on the Sill et al. . . . . . . . . .

1.10 Kinematic coverage of GMp data (Red) compared to SLAC data [7] (black) in

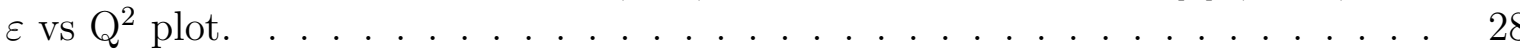

2.1 Layout of the CEBAF accelerator facility. . . . . . . . . . . . . . . 30 
2.2 Schematic view of experimental apparatus consisting two high resolution spectrometers (HRS) on either side of the beam line. The front view of the spectrometer is shown in Fig. 2.11. The scattered electrons are detected in two HRS's: LHRS and RHRS. . . . . . . . . . . . . . . . . . . . 32

2.3 Layout of Hall A Beamline components. . . . . . . . . . . . . . . . . . . . 33

2.4 Hall A arc schematic. . . . . . . . . . . . . . . . . . . . . . 34

2.5 Schematic of beam position measurement. . . . . . . . . . . . . . . . . 35

2.6 BPM coordinates system are shown by dotted lines (left) and a Harp (right) consisting three thin wires. The BPM coordinate system is rotated by $45^{\circ}$ with respect to nominal hall coordinate system. . . . . . . . . . . . . . . 36

2.7 The density of the events vs beam position projected to the target from BPM. 37

2.8 Schematic of HallA beam current measurement. . . . . . . . . . . . . . . . 38

2.9 Hall A target scattering chamber. Cryotarget loops on top and solid targets on the bottom are visible [9]. . . . . . . . . . . . . . . . . . . . 40

2.10 Target lifter GUI (Graphic User Interface) indicating positions of various targets. 41

2.11 Magnet configuration of the Hall A HRS spectrometers showing the sizes and locations of the dipole and the quadrupoles [10]. . . . . . . . . . . . . . . . . 42

2.12 Schematic of HRS Detector stack. . . . . . . . . . . . . . . . . . . . 44

2.13 Schematic diagram of the VDC wire planes showing a side view of the VDC pair (right figure) and the orientation of the wires relative to the central ray (left figure). Figure is taken from [1] . . . . . . . . . . . . . . . . . 45

2.14 Hall Coordinate system (top view) . . . . . . . . . . . . . . . . 46

2.15 Top view of the Target Coordinate system. $\mathrm{L}$ is the distance from the hall center to the sieve plane, while D is miss-pointing.

2.16 Detector coordinate system (top and side views). The intersection point of the wire 184 of the U1 plane and the perpendicular projection of wire 184 of the V1 plane onto the U1 plane is shown in the top view.

2.17 Schematic diagram of three U planes of straw tubes. Here $\mathrm{x}$ is along dispersive direction of HRS and $\mathrm{z}$ is along the HRS central ray. The $\mathrm{V}$ planes are located at the bottom of the straw frame not shown on the graph. . . . . . . . . . . 
2.18 Layout of the $\mathrm{s} 0$ scintillator plane. . . . . . . . . . . . . . . . . . 52

2.19 Layout of the $\mathrm{s} 2 \mathrm{~m}$ scintillator plane. . . . . . . . . . . . . . . . 53

2.20 Layout of the $\mathrm{CO}_{2}$ Gas Cherenkov detector. Figure is taken from [12]. . . . . . 54

2.21 Number of photoelectrons collected in the gas cherenkov detector. Red line represents the cut to select good electrons for Fall 2016 LHRS runs. . . . . . . 55

2.22 HAll A HRS calorimeter detector geometry. Particle enters through the bottom of figure. . . . . . . . . . . . . . . . . . . . 56

2.23 The total energy deposited in the calorimeter per unit momentum. The electron

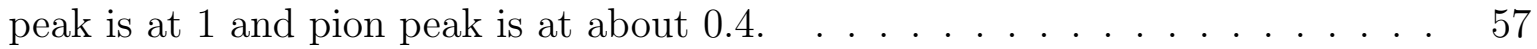

2.24 Schematics of Hall A DAQ system. . . . . . . . . . . . . . . . . . . 58

2.25 Schematics for Hall A trigger system. . . . . . . . . . . . . . . . . 59

3.1 Flow chart describing cross section extraction. . . . . . . . . . . . 63

3.2 Top: Harp wires response versus stepper motor position in mm. The peaks in the output of harp indicate points where the harp wires crossed the beam line. Bottom: Scatter plots of the BPM position in $\mathrm{y}$ vs. $\mathrm{x}$ for runs in the bulls eye scan

3.3 Top two plots are the fit results of LHRS BPM A positions vs corresponding Harp positions after calibration, whereas the lower two plots are the residual vs BPM positions . . . . . . . . . . . . . . . . . . . .

3.4 The bottom panel shows injected current vs entry number and the top panel shows the corresponding Unser current for those entries during Unser calibration. The time interval for each current-off and current-on period was 1 minute.

3.5 Left: The known injected current plotted as a function of offset subtracted Unser frequency. Right: The residual of the Unser current and the injected current source plotted, as a function of injected current. . . . . . . . . . . .

3.6 Figure shows an example plot of BCM u1 rate versus event number during BCM calibration. The time interval for each beam off and beam on period was 1 minute. 70

3.7 Top left panel shows the Unser current plotted against the BCM frequency. Top right shows the residual of the $\mathrm{BCM}$ current as given by $\mathrm{BCM}$ current obtained from the fit and the calculated Unser current plotted as a function 
BCM Current. Bottom left panel shows the percentage residual plotted against Unser current. The bottom right panel shows the residual versus time. . . . . . 71

3.8 Charge accumulated by receiver $\mathrm{u} 1$ divided by charge accumulated by receiver d1 plotted against run number. The blue points are the average currents as indicated by the scale on the right. . . . . . . . . . . . . . . . .

3.9 BCM Gains and offsets versus calibration run number showing the stability during the experiment. Calibration number 1 through 5 are from Spring of 2016, while calibration numbers 6 through 8 are from the Fall of 2016 and the calibration number 8 is the global as noted on the upper left panel.

3.10 Fall 2016 global BCM global calibration results for receiver u1. Top left panel shows the Unser current plotted against the BCM frequency. Top right shows the residual of the BCM current as given by BCM current obtained from the fit and the calculated Unser current plotted as a function BCM Current. Bottom left panel shows the percentage residual plotted against Unser current. The bottom right panel shows the residual versus time.

3.11 The rate for Trigger 1 versus entry number used to select entries when the beam was on. The region between the vertical line of a particular color denote the region of good entries when current was stable and not ramping.

3.12 The number of photoelectrons in Cherenkov as a function of energy deposited in the calorimeter normalized by momentum showing the selection cuts for good electrons. Everything to the right of the vertical red line in $E_{\text {cal }} / p$ and above the horizontal red line in Cherenkov number of photoelectrons represents the good electron events. . . . . . . . . . . . . . . . . . . .

3.13 Preliminary results of boiling studies on carbon (blue) and LH2 (red) targets. The top plot is showing charge normalized yield vs beam current and the bottom plot is showing scaler rate vs beam current prior to being corrected for the measured offset current. . . . . . . . . . . . . . . . . . . . . . . .

3.14 Scaler rate divided by current for T1 trigger as a function of beam current for carbon target. The systematic shape expected for an absolute current offset is fit to the data (given by red line) and yields a current offset of $0.13 \mu \mathrm{A}$. . . .

3.15 Results of boiling studies on carbon (blue) and LH2 (red) targets, after correcting for the offset in beam current. . . . . . . . . . . . . . . . . . . .

3.16 Charge normalized yield (top) and scaler rate normalized to current (bottom) versus beam current and fit to absolute current offset using the fit as discussed in Eq. 3.9 for the carbon target from the final analysis of B. Schmookler [13]. . 
3.17 The final results of boiling study carbon target showing both yield (top) and scaler (bottom) analysis [13] after correcting for the current offset. . . . . . .

3.18 Final results of the boiling study on LH2 target showing both yield (top) and scaler analysis (bottom) after correcting for the current offset from B. Schmookler [13].

3.19 Number of tracks reconstructed by the VDC for good electron events and the green distribution represents one track events.

3.20 The particle beta distribution formed from the scintillator timing information and path-length of the scintillator-based track. The events in the blue distribution shows all trigger one events. The green events the remaining events after the application of the PID cuts. The events between red lines represents good electron events, figure from Ref. [1]. . . . . . . . . . . . . . . .

3.21 Left: VDC single cluster efficiency in X bins in the focal plane transport coordinate system using the non-VDC based track for an inelastic run on the LHRS. Right: VDC single cluster efficiency in $\mathrm{Y}$ bins in the focal plane transport coordinate system. Figure is reproduced from Ref. [1]. . . . . . . . . . . .

3.22 Track $y$ in the focal plane transport coordinate system projected to VDC 1 indicating that more than $99 \%$ of events are in the region between the two red lines.

3.23 Top and bottom left panels shows the electron and pion sample selected in calorimeter to study the gas Cherenkov efficiency, where the events inside the red lines are the electrons and the events inside blue rectangle are the pions. The top left panel shows the number of photo-electrons collected in the gas Cherenkov for the electron(red) selected in top left panel. The last panel plot shows the Cherenkov cut efficiency for the electron (red) of $99.91 \%$ and pion rejection percentage (blue) of $99.96 \%$ selected in top left plot (These are the preliminary results for one of the low $Q^{2}$ Spring 2016 RHRS kinematics presented to outline the procedure. The final results for all Fall 2016 kinematics were performed by B. Schmookler [1] based on this procedure). . . . . . . . . . . . . . . . . . 95

3.24 Electron and pion cherenkov cut efficiencies as a function of ADC sum. . . . . 96

3.25 Event samples selected in Cherenkov to determine calorimeter detection and cut efficiencies

3.26 Grid showing electron cut efficiencies(red) and pion rejection efficiencies(blue) on the total energy deposited in the calorimeter and the energy deposited in layer 1 
3.27 Schematic of multi-foil carbon target and Sieve collimator [14] used for Fall 2106 optics optimization. . . . . . . . . . . . . . . . 104

3.28 Target y distribution after optimization. The blue vertical lines indicate the true foil target positions and Delta is the difference between the data Gaussian fitting center and real position. . . . . . . . . . . . . . . 107

3.29 Optimized reconstructed sieve pattern for five foils of multi-foil carbon target. The crosses indicate the average of front and back hole centers. . . . . . . . . 108

3.30 The position distribution in the horizontal direction with the Gaussian fitting of the one of the rows of sieve holes in the foil target. . . . . . . . . . . 108

3.31 The distribution of relative momentum for delta scan runs after optimization. The red line indicates the expected $\delta$ and $\Delta$ is the difference between reconstructed and expected $\delta \ldots \ldots \ldots$. . . . . . . . . . . . . 109

3.32 Reconstructed invariant mass shift at large $x_{t a r}^{\prime}$ for kinematics K1-1 with optimized optics. . . . . . . . . . . . . . . . . . . 110

3.33 The geometry of the target and beam positions. . . . . . . . . . . . . . 112

3.34 Determination of target $z$ and spectrometer offsets. . . . . . . . . . 113

3.35 Feynman diagrams for the elastic e-p scattering including the Born and lowest order radiative correction graphs $[15] . \ldots \ldots \ldots$

3.36 Schematic of radiative energy loss in elastic electron-proton scattering . . . . . 117

3.37 Feynman diagrams for the elastic e-p scattering including lowest order radiative correction graphs $[15] . \ldots \ldots \ldots \ldots$. . . . . . . . . . . . . . . . . . . . . . . . .

3.38 Data vs MC comparison plots of target quantities for kinematics K3-4 with nominal tune. The red histograms represents MC, black distributions represents background subtracted data and blue histogram represents the background. . . 122

3.39 Data vs MC comparison plots for K3-4 kinematics with optimized forward tunes in red. The black histogram represents background subtracted data, green histogram represents MC for nominal tune and blue histogram represents background. 123

3.40 Relative field per unit momentum versus read back current showing saturation above the set current of 550 A. . . . . . . . . . . . . . . 125

3.41 Target Z-vertex versus $y_{t a r}^{\prime}$ used to estimate the true $\int$ Bdl reduction. $\quad . . .126$ 
3.42 Reconstructed $\mathrm{W}$ and z-vertex resolution comparisons for nominal and saturation corrected optics. . . . . . . . . . . . . . . . . . 128

3.43 Data vs MC comparison for estimated Q1 $\int$ Bdl tune of $0.9315 \mathrm{Tm}$ (baseline tune). . . . . . . . . . . . . . . . . . . . . 129

3.44 Data vs MC comparison for re-tuned $\int \mathrm{Bdl}$ of $0.925 \mathrm{Tm} . \quad$. . . . . . . . . . . 130

3.45 Software cuts on sieve collimater used to study spectrometer acceptance. . . . 133

4.1 Normalized yield $\mathrm{w}$ distribution for LH2 target. The red solid line is the MC normalized yield, black points are the background subtracted data, and the blue solid line is the events from Aluminum windows of the LH2 target. . . . . . . . 138

4.2 Data yield vs MC yield comparison of kinematics K1-1. The red line shows the MC normalized yield, black points are the background subtracted data, and the blue line shows the events from aluminum windows of the LH2 target. . . . . .

4.3 Data yield vs MC yield comparison of the validation kinematics K1-1 after increasing field of Q2 by $0.9 \%$. The red line shows the MC normalized yield, black points are the background subtracted data, and the blue line shows the events from aluminum windows of the LH2 target. . . . . . . . . . . . . . . . . 141

4.4 Data yield vs MC yield of kinematics K3-4 after increasing the field of Q2 by $0.9 \%$. The red line shows the MC normalized yield, black points are the background subtracted data, and the blue line shows the events from aluminum windows of the LH2 target. . . . . . . . . . . . . . . . . . . . . . .

4.5 Data yield vs MC yield comparison of kinematics K3-8 after increasing field of Q2 by $0.9 \%$. The red line shows the MC normalized yield, black points are the background subtracted data, and the blue line shows the events from aluminum windows of the LH2 target. . . . . . . . . . . . . . . . . . . . . . . . . 143

4.6 Data yield vs MC yield comparison of kinematics K4-11 after increasing field of Q2 by $0.9 \%$. The red line shows the MC normalized yield, black points are the background subtracted data, and the blue line shows the events from aluminum windows of the LH2 target. . . . . . . . . . . . . . . . . . . . . . . . . 145

4.7 Left: Liquid hydrogen charge-normalized yield binned on 2-D grid in $\delta$ and $\theta$ bins Right: Dummy charge-normalized yield binned on 2-D grid in $\delta$ and $\theta$ bins. 147

4.8 Left: Generated $\delta$ vs generated $\Delta \theta$. Right: reconstructed $\delta$ vs reconstructed $\Delta \theta$ in $\delta-\Delta \theta$ space. 
4.9 Left: HRS acceptance in $\delta$ - $\Delta \theta$ space. Right: HRS effective solid angle plotted in $\delta-\Delta \theta$ space. . . . . . . . . . . . . . . . . . . . . . . . 149

4.10 Left: Acceptance corrected charge normalized yield $\delta$ - $\Delta \theta$ space. Right: Acceptance corrected charge normalized yield in $\Delta \theta \ldots \ldots \ldots$. . . . . . . 150

4.11 Extracted cross section for each $\Delta \theta \ldots \ldots \ldots \ldots$. . . . . . . . . . 151

4.12 Extracted cross section in each $\Delta \theta$. . . . . . . . . . . . . . . . . . . . 152

5.1 The results of extracted $e-p$ cross sections divided by the cross section calculated in the OPEA assuming a dipole form for both $G_{E}$ and $G_{M}$ as a function of $Q^{2}$. The inner error bar in GMp data are statistical and outer error bars are combined point-to-point systematic and statistical uncertainties. . . . . . . . . 154

5.2 The results of model dependent extraction of GMp as a function of $Q^{2}$. The uncorrelated uncertainties reflect the point-to-point systematic and the statistical uncertainties added in quadrature, while the correlated uncertainty shown as the green histogram at the bottom are calculated assuming a $20 \%$ uncertainty on the $G_{E} / G_{M}$ value used to extract GMp from the cross section data. . . . . 157

5.3 Reduced cross section normalized by $G_{D}^{2}$ versus $\varepsilon$ at $Q^{2}=1.7 \mathrm{GeV}^{2}$. . . . . . 158

5.4 Reduced cross section normalized by $G_{D}^{2}$ versus $\varepsilon$ at $Q^{2}=5.0 \mathrm{GeV}^{2}$. . . . . . 158

5.5 Reduced cross section normalized by $G_{D}^{2}$ versus $\varepsilon$ at $Q^{2}=5.8 \mathrm{GeV}^{2}$. . . . . . 159

5.6 Reduced cross section normalized by $G_{D}^{2}$ versus $\varepsilon$ at $Q^{2}=7.0 \mathrm{GeV}^{2}$. . . . . . 159

5.7 Reduced cross section normalized by $G_{D}^{2}$ versus $\varepsilon$ at $Q^{2}=9.1 \mathrm{GeV}^{2}$. . . . . . 160 


\section{CHAPTER 1 INTRODUCTION}

Since the development of modern physics, scientists have tried to discover the structure of nucleons (protons and neutrons), which are the building blocks of atomic nuclei. The $\sim 2.8$ times bigger proton magnetic moment than the nuclear magneton (expected from a point particle) measured by Stern in 1933 provided the first clue that the proton was not a point-like particle. Almost 20 years later the first measurement by Hofstadter gave hints on the proton's internal structure. Hofstadter [24] combined the Mott cross section (the cross section for point like particle) with an internal charge density to describe the deviation from a point-like particle experimentally. Thus, the nucleons, once thought to be the smallest building blocks of matter, turned out to be composed of even smaller objects, which are now known to be quarks and gluons. Understanding how the nucleon is built in terms of these fundamental particles is one of the most important questions in modern nuclear physics, which can be explained by a theory called quantum chromodynamics (QCD). This is a theory of strong interactions, a fundamental force describing the interaction between quarks and gluons.

The basic reasons why the electromagnetic interaction is an important tool for studying the the picture of nucleon is: Quantum electrodynamics (QED) is a known interaction and for the electro-magnetic coupling constant $\alpha_{e m}=1 / 137$, perturbation theory is valid. By contrast, the strong coupling constant for QCD renormalization scale $\Lambda$

$$
\alpha_{s} \propto \frac{1}{\ln \left(\frac{Q^{2}}{\Lambda^{2}}\right)}
$$


is small only at high energy in the asymptotic freedom. Since the interaction between electron and target is very weak, this makes it easy for an electron to probe the entire target nucleon. In this process, the interaction between the electron and the target can be described by the exchange of a virtual photon which interacts with the charge density and the electromagnetic currents of the target nucleon. In elastic electron-nucleon scattering via photon exchange, the structure of the nucleon is characterized in terms of the electric and magnetic form factors, which are sensitive to the distribution of charge and current. From the measurement of the cross-section for electron scattering at different final electron energies and scattering angles, one can describe the response of the nucleons to the electromagnetic probe.

In order to obtain a deeper understanding of the internal structure of the proton, the data on the electromagnetic form factors play an important role. These data may be useful to establish the details of the interaction of quark and gluons constituting the proton as well as criteria to test the various theoretical models within QCD. Most early form factor data were analyzed by the Rosenbluth separation method where the form factors are extracted by looking at the cross section as a function of $Q^{2}$ and $\varepsilon$. At large $Q^{2}$ the contribution from the electric form factor is small. So this technique has reduced the sensitivity to $G_{E}$, and the ratio of $G_{E}$ to $G_{M}$ changes only slightly and is close to unity.

The recent experiment at Jefferson Lab, utilizing the polarization transfer method reveals most surprising that $G_{E}$ decreases more rapidly than $G_{M}$ at large $Q^{2}$. In this method, the ratio of $G_{E}$ to $G_{M}$ is expressed in the Born approximation in terms of the ratio of transverse and longitudinal components of recoil polarization and strongly depends on $Q^{2}$. These two methods, Rosenbluth and polarization transfer, disagree significantly even in the region where they both provide precise measurements.

It has been assumed that this contradiction can be removed by taking into account the hard part of the contribution of two-photon exchange to the cross section in elastic electronproton scattering (this study is detailed in a later section). Recent theoretical studies of two-photon exchange (TPE) suggested that TPE effects give rise to a significant angular 


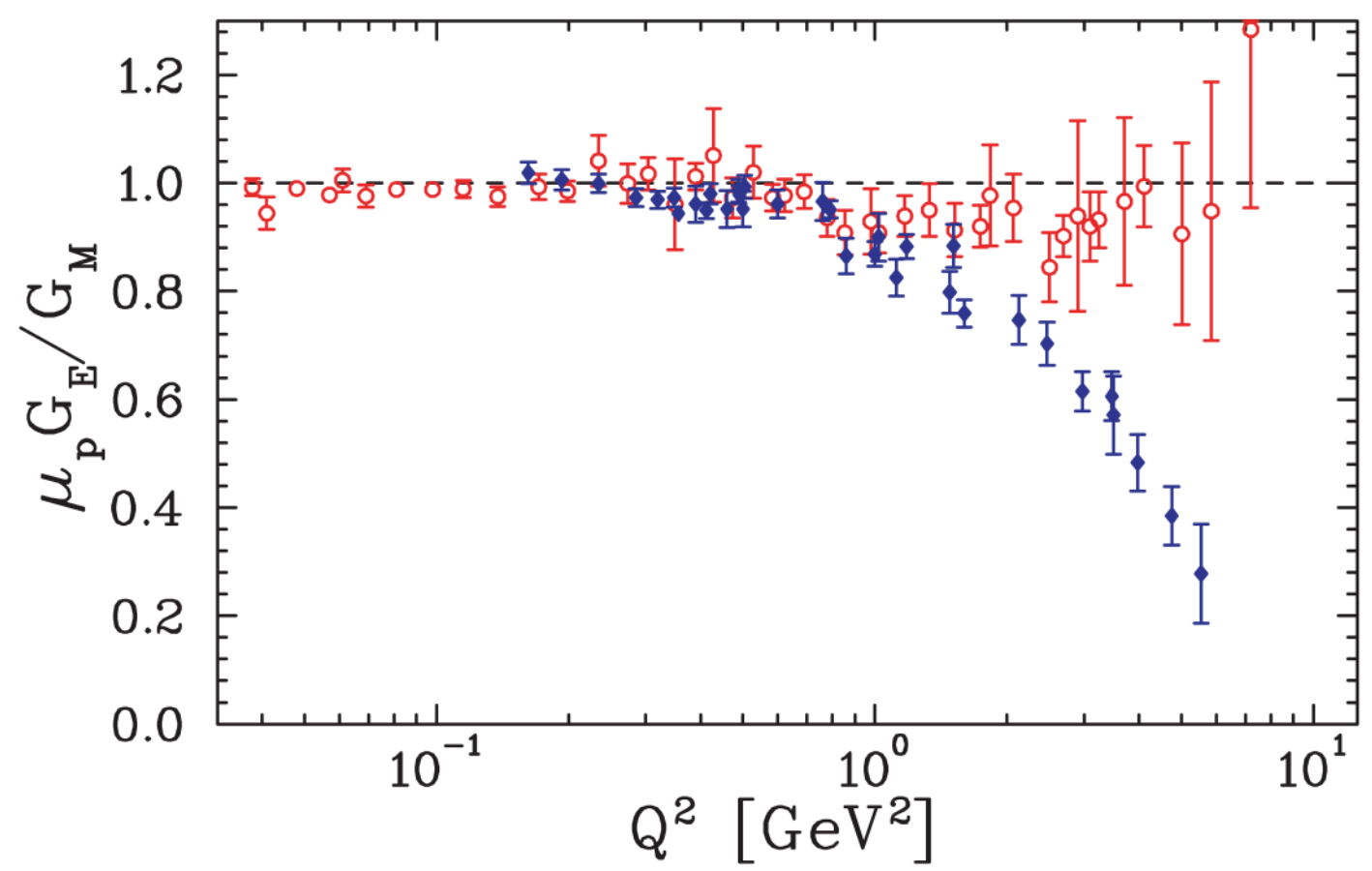

Figure 1.1. Ratio of the proton form factors as a function of $Q^{2}$ according to data obtained by the Rosenbluth method in (red points) and by the polarization-transfer measurements (blue points) in single photon exchange approximation. Plot is from Ref. [2].

dependent correction to the elastic cross section which can lead to a large correction to the extracted ratio $G_{E} / G_{M}$ in the Rosenbluth separation method [2].

The GMp experiment was proposed to perform a high precision measurement of the elastic $e-p$ cross section to extract the proton magnetic form-factor in the $Q^{2}$ range from 1 to $17 \mathrm{GeV}^{2}$ with precision of better than $2 \%$. Existing data on $G_{M}$ at high $Q^{2}$ have large uncertainties, and also have uncertainties related to TPE effects and the contribution from $G_{E}$, limiting the ability to extract information on the $Q^{2}$ dependence. GMp was proposed to significantly improve the statistical and systematic uncertainties on the cross section. As the measurements of this experiment were taken at lower $\varepsilon$ (virtual photon polarization) as shown in Fig. 1.10, the contributions from $G_{E}^{p}$ are significantly smaller than for the large $Q^{2}$ SLAC data where $G_{E}^{p}$ may be negative and large. In addition, the GMp data will provide better constraints on the high $Q^{2}$ behavior of the form factor allowing for higher precision tests of models of the nucleon form factor. Finally, this experiment measured cross sections 
at kinematics where TPE corrections will be very similar to what is seen in other experiments at JLab which will minimizes the assumptions on TPE effects.

\section{$1.1 \quad$ Form Factors}

In the electron-proton scattering process, the final state of the nucleon remains the same, but with a finite recoil. Form factors are the physical observables used to describe the spatial extent of the nucleon. For an extended object like the proton having a matter density $\rho(r)$, the form factor $F(q)$ is

$$
F(\vec{q})=\int d^{3} r e^{i \vec{q} \cdot \vec{r}} \rho(\vec{r})
$$

This implies that for $q=0$

$$
F(0)=1
$$

which gives the total charge. The cross section for an extended object is modified by the form factor as

$$
\frac{d \sigma}{d \Omega}(\text { extended }) \approx \frac{d \sigma}{d \Omega}_{(\text {point-like })}|F(\vec{q})|^{2}
$$

For electron-proton scattering, two form factors are required to describe their structure: the Dirac form factor $F_{1}$ to describe the distribution of the electric charge, and the Pauli form factor $F_{2}$ to describe recoil of the proton. They are distinguished according to their helicity states, which is defined as the projection of spin of the electron along its direction of motion. In the non-relativistic case, the Fourier transform of electric charge distribution of the proton corresponds to the electric form factor $\left(G_{E}^{p}\right)$, whereas if the target has extended magnetic moment distribution, the Fourier transform of the magnetic distribution of the proton is referred as magnetic factor $\left(G_{M}^{p}\right)$. 
In the case of an extended charge distribution $\rho(\vec{r})=\rho_{0} e^{-r / a}$ in Breit frame, the resulting form factor is the dipole form factor $G_{D}\left(Q^{2}=\left(1+a^{2} Q^{2}\right)^{-2}\right.$ where a is the radius of the proton. Experimental observations have shown that both electric and magnetic form factors can be described to a good approximation by the dipole form factor. More generally, if the charge and magnetic distributions are the same then the electric and magnetic form factors in the non-relativistic limit are related by

$$
\begin{aligned}
G_{E}^{p}\left(Q^{2}\right) & =G^{D}\left(Q^{2}\right) \\
& =\frac{G_{M}^{p}\left(Q^{2}\right)}{\mu_{p}}
\end{aligned}
$$

And the term

$$
G_{E}^{p}\left(Q^{2}\right)=\frac{G_{M}^{p}\left(Q^{2}\right)}{\mu_{p}}
$$

is called form factor scaling.

\subsection{Hadronic Currents}

In the Breit frame (also known as infinite-momentum frame), elastically scattered electrons transfer momentum $\vec{q}_{B}$ as a result of which the proton undergoes a change from $-\vec{q}_{B} / 2$ to $+\vec{q}_{B} / 2$. Thus, the initial and final nucleon momenta are equal and opposite in this frame resulting in a simplified expression for the hadronic current. Using the Gordon identity

$$
\bar{u}\left(p^{\prime}\right) \gamma^{\mu} u(p)=\bar{u}\left(p^{\prime}\right)\left[\frac{p^{\prime \mu}+p^{\mu}}{2 M_{p}}+\frac{i \sigma^{\mu u} q_{u}}{2 M_{p}}\right] u(p)
$$


This allows the $p+p^{\prime}$ term to be exchanged for the one involving the term $\sigma^{\mu u} q_{u}$. With this the hadronic current in terms of the Dirac and Pauli form factors will take the form:

$$
\mathcal{J}^{\mu}=\bar{u}\left(p^{\prime}\right)\left[\left(F_{1}+\kappa F_{2}\right) \gamma^{\mu}-\frac{\left(p^{\prime}+p\right)^{\mu}}{2 M_{p}} \kappa F_{2}\right] u(p)
$$

Hence, the time and spatial components of the hadronic currents are given by

$$
\begin{aligned}
\mathcal{J}^{0} & =i e \bar{u}\left(p^{\prime}\right)\left[\left(F_{1}+\kappa F_{2}\right) \gamma^{0}-\frac{E_{p B}}{M_{p}} \kappa F_{2}\right] u(p), \\
\overrightarrow{\mathcal{J}} & =i e\left(F_{1}+\kappa F_{2}\right) \bar{u}\left(p^{\prime}\right) \vec{\gamma} u(p),
\end{aligned}
$$

where, $E_{p B}$ is the photon energy in the Breit frame and $\vec{\gamma}=\left(\gamma^{1}, \gamma^{2}, \gamma^{3}\right)$. As $\bar{u}\left(p^{\prime}\right)=u^{\dagger}\left(p^{\prime}\right) \gamma^{0}$, such that

$$
\mathcal{J}^{0}=i e\left[\left(F_{1}+\kappa F_{2}\right) \gamma^{0} u^{\dagger}\left(p^{\prime}\right) u(p)-\frac{E_{p B}}{M_{p}} \kappa F_{2} u^{\dagger}\left(p^{\prime}\right) \gamma^{0} u(p)\right]
$$

where

$$
u(p)=\left(\begin{array}{l}
\sqrt{(p . \sigma)} \chi \\
\sqrt{(p . \bar{\sigma})} \chi
\end{array}\right)
$$

represents the Dirac four-spinnors and

$$
\gamma^{0}=\left(\begin{array}{ll}
0 & 1 \\
1 & 0
\end{array}\right)
$$


Using $u(p)$ and $\gamma^{0}$ in Eq. 1.10 yields

$$
\begin{aligned}
\mathcal{J}^{0} & =i e\left(F_{1}+\kappa F_{2}\right) \chi^{\prime \dagger}\left(\sqrt{\left(p^{\prime} . \sigma\right)}, \sqrt{\left(p^{\prime} . \bar{\sigma}\right)}\right)(\sqrt{(p . \sigma)} \chi \sqrt{(p . \bar{\sigma})} \chi) \\
& -i e \frac{E_{p B}}{M_{p}} \kappa F_{2} \chi^{\dagger}\left(\sqrt{\left(p^{\prime} \cdot \sigma\right)}, \sqrt{\left(p^{\prime} \cdot \bar{\sigma}\right)}\right)\left(\begin{array}{ll}
0 & 1 \\
1 & 0
\end{array}\right)\left(\begin{array}{c}
\sqrt{(p . \sigma)} \chi \\
\sqrt{(p . \bar{\sigma})} \chi
\end{array}\right) .
\end{aligned}
$$

Using the identities

$$
\begin{aligned}
M_{p} & =\sqrt{p^{\prime} \cdot \sigma} \cdot \sqrt{p^{\prime} \cdot \sigma} \\
& =\sqrt{p^{\prime} \cdot \bar{\sigma}} \cdot \sqrt{p^{\prime} \cdot \bar{\sigma}} \\
E_{p B} & =\frac{\sqrt{p \cdot \sigma} \cdot \sqrt{p^{\prime} \cdot \bar{\sigma}}+\sqrt{p^{\prime} \cdot \bar{\sigma}} \cdot \sqrt{p \cdot \sigma}}{2}
\end{aligned}
$$

with

$$
\tau=\frac{Q^{2}}{4 M_{p}^{2}}=\frac{q_{B}^{2}}{4 M_{p}^{2}}=\frac{E_{p B}^{2}-M_{p}^{2}}{M_{p}^{2}} .
$$

The time component will be,

$$
\begin{aligned}
\mathcal{J}^{0} & =2 i e M_{p} \chi^{\prime} \dagger \chi\left(F_{1}-\tau \kappa F_{2}\right) \\
& =2 i e M_{p} \chi^{\prime \dagger} \chi G_{E}
\end{aligned}
$$

and the vector current $\overrightarrow{\mathcal{J}}$

$$
\begin{aligned}
\overrightarrow{\mathcal{J}} & =-e \chi^{\prime \dagger}\left(\vec{\sigma} \times \overrightarrow{q_{B}}\right) \chi\left(F_{1}+\kappa F_{2}\right) \\
& =-e \chi^{\prime \dagger}\left(\vec{\sigma} \times \overrightarrow{q_{B}}\right) \chi G_{M} .
\end{aligned}
$$


Using these results Sachs [25] form factors can be interpreted in the Breit frame. In this frame $G_{E}$ and $G_{M}$ are expressed as:

$$
\begin{aligned}
G_{E, M}\left(Q^{2}\right) & =\int \rho(\vec{r})_{E, M} e^{i \vec{q} \cdot \vec{r}} d^{3} r \\
& =\int \rho(\vec{r})_{E, M} d^{3} r-\frac{q^{2}}{6} \int \rho(\vec{r})_{E, M} r^{2} d^{3} r .
\end{aligned}
$$

In this expansion the first term gives the total electric charge or magnetic current and the second term provides the corresponding root-mean-squared radius of the nucleon [26]. But, this simplified interpretation is complicated by a Lorentz contraction of the nucleon in the direction of motion which leads to non-spherical charge density distributions that complicate the naive Fourier transform interpretation of these form factors.

\section{$1.3 \quad$ Elastic Scattering}

Electron scattering is an important tool for studying the structure of nucleons. This scattering process is dominated by electromagnetic interaction where a single virtual photon is exchanged. By measuring the cross-section for electron scattering at various final electron energies and scattering angles, one can map out the response of the nucleon to the electromagnetic probe. Let us consider an elastic scattering between a charged electron and proton through the exchange of one photon represented by a Feynman diagram shown in Fig. 1.2. Here the incident and outgoing electrons have 4-momenta $k=(E, \vec{k})$ and $k^{\prime}=\left(E, \vec{k}^{\prime}\right)$, and the initial and final proton 4-momenta are p and p', respectively. The 4-momentum of the exchanged virtual photon is

$$
q=k-k^{\prime}=p-p^{\prime}
$$

The square of the four momentum transfer of photon is $q^{2}=-4 E E^{\prime} \sin ^{2} \theta / 2$, neglecting the electron mass. In the Breit frame, where the energy transfer $q^{0}=\nu=0$ and $q^{2}=-\vec{q}^{2}=Q^{2}$, the elastic scattering condition in the lab frame gives, 


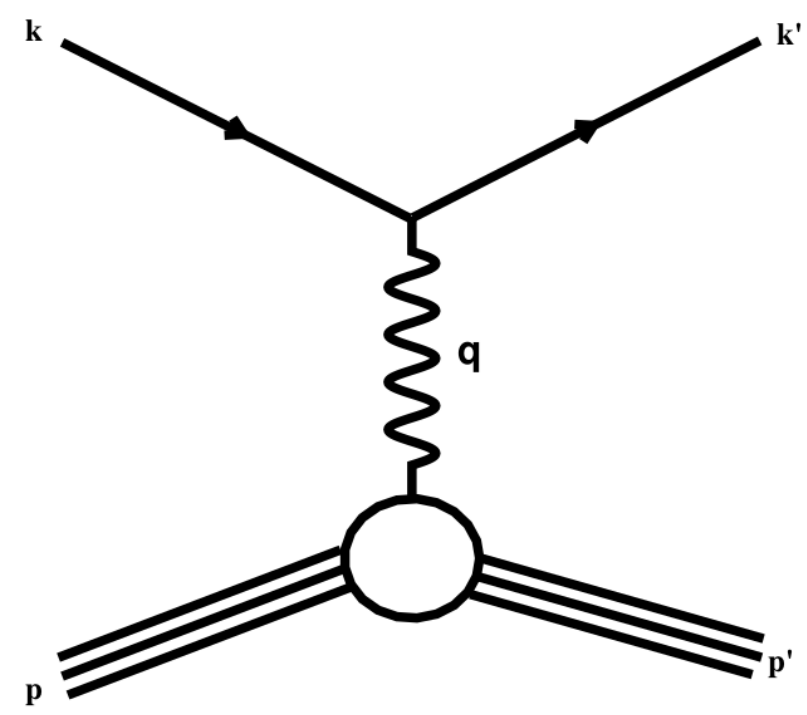

Figure 1.2. Feynman diagram for electron proton elastic scattering

$$
\begin{aligned}
p^{\prime 2} & =(p+q)^{2}=M^{2} \\
& =M^{2}+2 p \cdot q+q^{2} \\
& =M^{2}+2 M \nu+q^{2} .
\end{aligned}
$$

The S-matrix element for electron-photon vertex $-i e \gamma^{\mu}$ is

$$
\begin{aligned}
S & =(2 \pi)^{4} \delta^{4}\left(k+p-p^{\prime}-k^{\prime}\right) \bar{u}\left(k^{\prime}\right)\left(-i e \gamma^{\mu}\right) u(k) \frac{-i}{q^{2}}<p^{\prime}\left|i e J^{\mu}\right| p> \\
& =-i(2 \pi)^{4} \delta^{4}\left(k+p-p^{\prime}-k^{\prime}\right) \mathscr{M} .
\end{aligned}
$$

$\mathscr{M}$ is invariant amplitude

$$
i \mathscr{M}_{f i}=(-i e) \bar{u}\left(k^{\prime}\right) \gamma^{\mu} u(k) \frac{-i}{q^{2}}(i e)<p^{\prime}\left|J^{\mu}\right| p>
$$


The elastic scattering cross section in terms of the invariant amplitude is

$$
d \sigma=\frac{(2 \pi)^{4} \delta^{4}\left(k+p-p^{\prime}-k^{\prime}\right)}{2 k^{0} 2 p^{0}\left|v_{2}-v_{1}\right|}\left|\mathscr{M}_{f i}\right|^{2} \prod_{f} \frac{d^{3} p_{f}}{2 E_{f}(2 \pi)^{3}}
$$

Here, $v_{1}$ is initial proton velocity, $v_{2}$ is the electron velocity, and $2 k^{0} 2 p^{0}\left|v_{2}-v_{1}\right|$ is invariant when boosted along the z-direction. In the lab frame, $k=E, p^{0}=M$, and integration is made over the proton momentum by writing the phase factor as $2 \pi \delta\left(p^{2}-M^{2}\right) d^{4} p^{\prime} /(2 \pi)^{4}$ to get

$$
d \sigma=\frac{|\mathscr{M}|^{2} 2 \pi \delta\left((q+p)^{2}-M^{2}\right)}{2 M E} \frac{d^{3} \vec{k}^{\prime}}{2 E^{\prime}(2 \pi)^{3}} .
$$

Integrating over $E^{\prime}=\left|\vec{k}^{\prime}\right|$, to obtains

$$
d \sigma=\frac{E^{\prime}|\mathscr{M}|^{2}}{2(2 \pi)^{2} E M^{2}\left(1+\frac{2 E}{M} \sin ^{2} \theta / 2\right)} d \Omega .
$$

Even though the proton is massive, its recoil effects cannot be neglected. This factor is relativistic in nature. In terms of electron kinematics this factor can be written as

$$
\frac{E^{\prime}}{E}=\frac{1}{1+\frac{2 E}{M} \sin ^{2} \frac{\theta}{2}}
$$

The invariant amplitude squared is given by

$$
\begin{aligned}
|\mathscr{M}|^{2} & =\frac{e^{4}}{Q^{4}} l^{\mu \nu} W_{\mu \nu} \\
& =\frac{e^{4}}{Q^{4}} l^{\mu \nu}<p\left|J^{\nu}\right| p^{\prime}><p^{\prime}\left|J^{\mu}\right| p>
\end{aligned}
$$

where $l^{\mu \nu}$ is the leptonic tensor such that

$$
l^{\mu \nu}=\bar{u}\left(k^{\prime}\right) \gamma^{\mu} u(k) \bar{u}(k)^{\nu} u\left(k^{\prime}\right)
$$


Average over the initial polarization and sumed over the final polarization states (for an unpolarized electron) yields

$$
l^{\mu \nu}=2\left(k^{\prime \mu} k^{\nu}+k^{\prime} k^{\mu}-g^{\mu \nu} k^{\prime} \cdot k\right) .
$$

The current density between the nucleon states defines two form factors as

$$
<p^{\prime}\left|J^{\mu}(0)\right| p>=\bar{u}\left(p^{\prime}\right)\left[F_{1}\left(Q^{2}\right) \gamma^{\mu}+F_{2}\left(Q^{2}\right) \frac{i \sigma^{\mu \nu} q_{\nu}}{2 M}\right] u(p)
$$

The Sachs [25] electric and magnetic form factors are defined as

$$
\begin{aligned}
& G_{M}\left(Q^{2}\right)=F_{1}\left(Q^{2}\right)+F_{2}\left(Q^{2}\right), \\
& G_{E}\left(Q^{2}\right)=F_{1}\left(Q^{2}\right)-\tau F_{2}\left(Q^{2}\right),
\end{aligned}
$$

where,

$$
\tau=\frac{Q^{2}}{4 M^{2}}
$$

When $Q^{2}=0$ :

$$
\begin{aligned}
& G_{M}(0)=F_{1}(0)+F_{2}(0)=\mu_{p}=2.793 \mu_{N}, \\
& G_{E}(0)=F_{1}(0)=1
\end{aligned}
$$

where, $\mu_{N}$ is the nuclear magneton. The hadronic tensor will be then

$$
W^{\mu \nu}=g^{\mu \nu} q^{2} G_{M}^{2}+p^{\mu} p^{\nu} \frac{G_{E}^{2}+\tau G_{M}^{2}}{1+\tau}
$$


As both tensors are symmetric and conserved, $q^{\mu} l_{\mu \nu}=q^{\mu} W_{\mu \nu}=0$, the elastic cross section in the lab frame in single photon approximation is given by

$$
\frac{d \sigma}{d \Omega}=\sigma_{M o t t}\left[\frac{G_{E}^{2}+\tau G_{M}^{2}}{1+\tau}+2 G_{M}^{2} \tan ^{2} \frac{\theta}{2}\right]
$$

where $\sigma_{\text {Mott }}$ is the cross section for a structure-less Dirac particle which is expressed as

$$
\sigma_{M o t t}=\frac{\alpha^{2} \cos ^{2} \frac{\theta}{2}}{4 E^{2} \sin ^{4} \frac{\theta}{2}} \frac{E^{\prime}}{E} .
$$

In terms of $G_{E}$ and $G_{M}$ the ep elastic cross section can be rewritten as

$$
\frac{d \sigma}{d \Omega}=\frac{\sigma_{M o t t}}{1+\tau}\left[G_{E}^{2}+\frac{\tau}{\varepsilon} G_{M}^{2}\right]
$$

where $\varepsilon^{-1}=1+2(1+\tau) \tan ^{2} \frac{\theta}{2}$ is the virtual photon polarization.

\subsection{Experimental Measurement of Form Factors}

There are two methods of elastic form factor measurement:

- Rosenbluth separation : At small $Q^{2}$ the magnetic form factor $G_{M}^{p}$ of the proton is suppressed and the cross section is dominated by the contribution of the electric form factor $G_{E}^{p}$ except as $\varepsilon$ close to zero. But at large $Q^{2}$ the electric form factor $G_{E}^{p}$ is suppressed and the cross section is dominated by the contribution of the magnetic form factor $G_{M}^{p}$. The fact that the magnetic form factor dominates at large $Q^{2}$, make it difficult to extract $G_{E}^{p}$ with high accuracy from the measured cross section at large $Q^{2}$. Similarly, highly accurate $G_{M}^{p}$ at small $Q^{2}$ is difficult to extract. Rewriting the expression of cross section in terms of the reduced cross section

$$
\sigma_{R}=\frac{d \sigma}{d \Omega} \frac{(1+\tau) \varepsilon}{\tau \sigma_{M o t t}}=G_{M}^{2}+\frac{\varepsilon}{\tau} G_{E}^{2}
$$


By measuring the reduced cross section at several $\varepsilon$ points for a fixed $Q^{2}$, a linear fit of $\sigma_{R}$ to $\varepsilon$ gives $G_{M}^{2}$ as the intercept and $G_{E}^{2} / \tau$ as the slope. This is known as the Rosenbluth separation method. Getting $G_{M}$ and $G_{E}$ at fixed $Q^{2}$, the ratio of electric to magnetic form factors of the proton $\frac{\mu_{p} G_{E}}{G_{M}}$ can be calculated for that $Q^{2}$ point.

- Recoil polarization technique: In the Rosenbluth separation method, $G_{M}$ dominates the cross section at all $\varepsilon$ values at large $Q^{2}$. Therefore, the uncertainty in $G_{E}$ increases with increasing $Q^{2}$. The recoil polarization technique has been introduced recently to get a more reliable measurement of $G_{E}$. In this technique, a longitudinally polarized electron beam is scattered by unpolarized protons resulting the recoil proton with polarization in the scattering plane. The polarization transverse and parallel to the momentum of the electron are given by

$$
\begin{aligned}
& I_{0} P_{t}=-2 \sqrt{(\tau(1+\tau))} G_{E} G_{M} \tan \frac{\theta}{2}, \\
& I_{0} P_{l}=\frac{E+E^{\prime}}{M} \sqrt{(\tau(1+\tau))} G_{M}^{2} \tan ^{2} \frac{\theta}{2},
\end{aligned}
$$

Where, $I_{0}=G_{E}^{2}+\frac{\tau}{\varepsilon} G_{M}^{2}$.

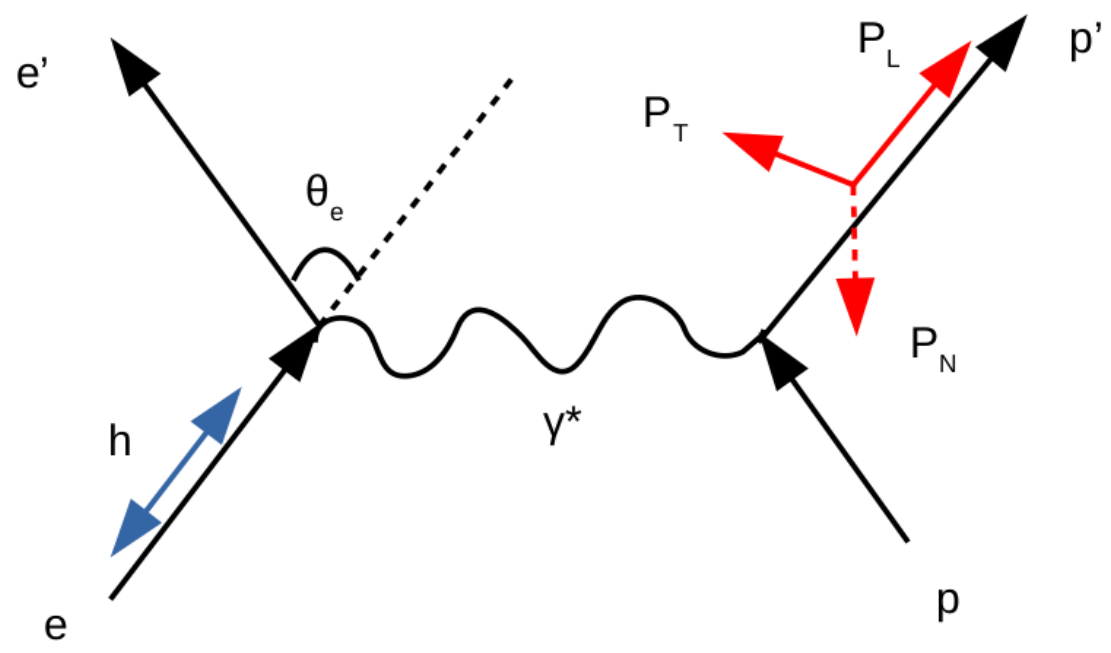

Figure 1.3. Schematic diagram for the recoil polarization. 
In this technique, the transverse and longitudinal polarization, $P_{t}$ and $P_{l}$ respectively, of the scattered proton are measured by a Focal Plane Polarimeter (FPP). Re-scattering the proton from a secondary target inside the FPP and measuring the azimuthal angular distribution gives those components of polarization. By forming the ratio of the transverse and longitudinal polarization, the ratio of $G_{E}$ to $G_{M}$ is given by

$$
\frac{G_{E}}{G_{M}}=-\frac{P_{t}}{P_{l}} \frac{E+E^{\prime}}{2 M} \tan \frac{\theta}{2} .
$$

Unlike the Rosenbluth Separation method which measures the cross-section at different kinematics, this technique gives the ratio $G_{E} / G_{M}$ directly. By measuring $G_{M}$ using the extracted cross-section, $G_{E}$ can be measured with smaller uncertainties.

\subsection{Two-photon Exchange for Unpolarized Scattering}

The first quantitative calculation of the effect of TPE on the ratio $G_{E} / G_{M}$ was done by Blunden [27]. Fig. 1.4 shows the contributions to elastic electron-proton scattering from one photon and two-photon exchanges with particle momenta as indicated. The Born amplitude for one photon exchange in the case of unpolarized scattering is given by

$$
\mathscr{M}_{0}=-i \frac{e^{2}}{q^{2}} \bar{u}\left(p_{3}\right) \gamma_{\mu} u\left(p_{1}\right) \bar{u}\left(p_{4}\right) \Gamma^{\mu}(q) u\left(p_{2}\right)
$$



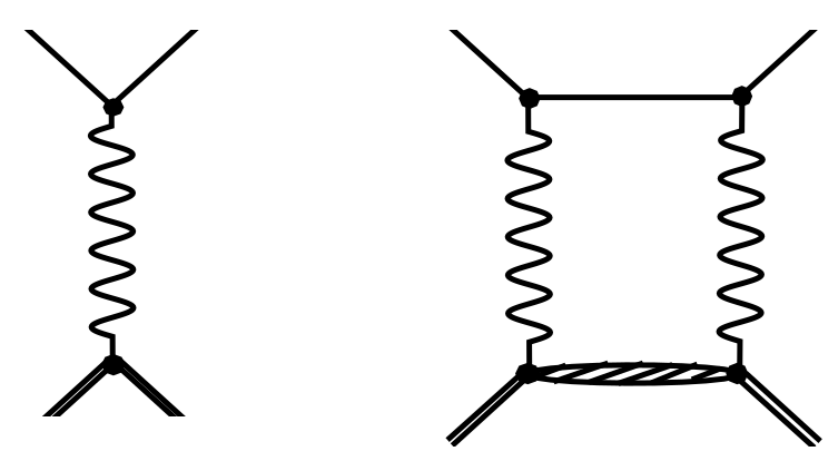

Figure 1.4. Feynman diagrams for electron-proton elastic scattering: one-photon exchange (Born approximation) and two-photon exchange scattering.

The differential scattering cross section in terms amplitude $\mathscr{M}_{0}$ is given by

$$
\frac{d \sigma_{0}}{d \Omega}=\left(\frac{\alpha}{4 M q^{2}}\right)^{2}\left|\mathscr{M}_{0}\right|^{2}
$$

where, $\alpha=e^{2} / 4 \pi$ is the fine structure constant. The elastic scattering cross section defined in Eq. 1.40 including the radiative corrections to order $\alpha$ will become

$$
\frac{d \sigma}{d \Omega} \rightarrow \frac{d \sigma}{d \Omega}\left(1+\delta_{r c}\right)
$$

where, $\delta_{r c}$ is calculated by using the one loop virtual corrections of order $\alpha$ and the inelastic bremsstrahlung cross section for real photon emission. The one loop correction amplitudes $\mathscr{M}_{1}$ can be expressed as the sum of a factorizable term $\left(\overline{\mathscr{M}_{0}}\right)$, and a non-factorizable term $\overline{\mathscr{M}_{1}}$ :

$$
\mathscr{M}_{1}=f\left(Q^{2}, \varepsilon\right) \mathscr{M}_{0}+\overline{\mathscr{M}}_{1}
$$


where, $\mathscr{M}_{o}$ is the born amplitude. The ratio of full cross section to the Born can be expressed as

$$
1+\delta_{r c}=\frac{\left|\mathscr{M}_{0}+\mathscr{M}_{1}\right|^{2}}{\left|\mathscr{M}_{0}\right|^{2}}
$$

Here, $\delta$ is given by

$$
\begin{aligned}
\delta_{r c} & =2 f\left(Q^{2}, \varepsilon\right)+\frac{2 \mathscr{R}\left\{\mathscr{M}_{0}^{\dagger} \mathscr{M}_{1}\right\}}{\left|\mathscr{M}_{0}\right|^{2}}, \\
& =\delta_{\text {soft }}+\delta_{\text {hard }},
\end{aligned}
$$

The soft terms in $\delta_{\text {soft }}$ include the electron vertex correction, vacuum polarization and the infrared divergent (IR) parts of the nucleon vertex and TPE corrections. The main contributions to the function $f\left(Q^{2}, \varepsilon\right)$ from the electron vertex, vacuum polarization, and proton vertex depend only on $Q^{2}$, and hence have no effect for the Rosenbluth separation, other than from an overall normalization. Therefore, only the IR-divergent TPE correction in the factorizable term can contribute to the $\varepsilon$-dependence of the cross section. The nonfactorizable term which depend on the hadronic structure are contained in $\mathscr{M}_{1}$ came from the TPE corrections and proton vertex. As the proton vertex correction has a weak $\varepsilon$ dependence, it will not affect much the Rosenbluth separation process. The cross section is a simple kinematic factor times the Born cross section in soft photon approximation if one of the two exchanged photons is taken to be on-shell. The corrections after the standard $\mathscr{O}(\alpha)$ radiative corrections that can introduce the weak $\varepsilon$ dependence, the non-factorizable term of the TPE contribution, is not taken into account in cross section extractions. The TPE correction $\delta_{2 \gamma}$ is given by the interference of the total TPE and Born amplitudes:

$$
\delta_{2 \gamma}=\frac{2 \mathscr{R}\left\{\mathscr{M}_{0}^{\dagger} \mathscr{M}_{2 \gamma}\right\}}{\left|\mathscr{M}_{0}\right|^{2}}
$$

Where, the TPE amplitude $\mathscr{M}_{2 \gamma}$ includes all possible off-shell intermediate states. 

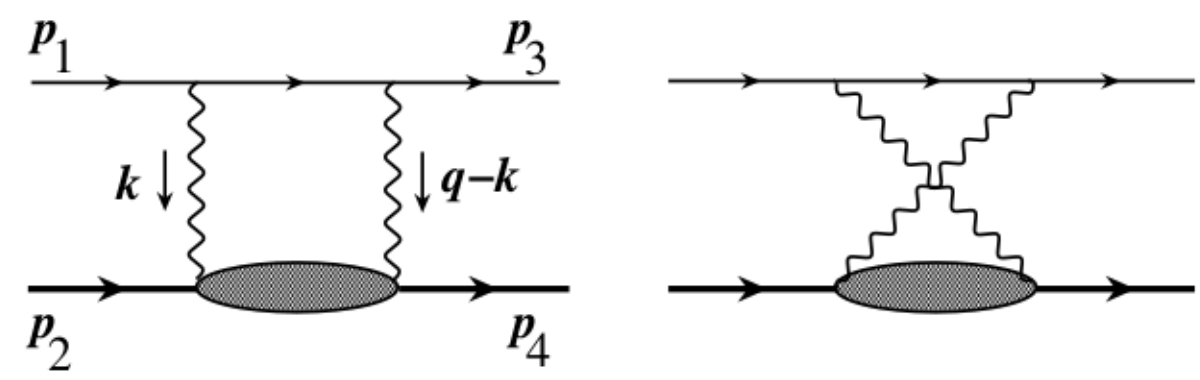

Figure 1.5. Feynman diagrams for electron-proton elastic scattering: two-photon exchange scattering.

The total IR divergent TPE contribution to the cross section in the target rest frame is given by

$$
\delta_{I R}=-\frac{2 \alpha}{\pi} \ln \left(\frac{E_{1}}{E_{3}}\right) \ln \left(\frac{Q^{2}}{\lambda^{2}}\right)
$$

The IR divergent contribution to the cross section introduced by Mo and Tsai [MT] Ref. [28] is given by

$$
\delta_{I R}(M T)=-\frac{2 \alpha}{\pi}\left[k\left(p_{1}, p_{2}\right)-k\left(p_{3}, p_{2}\right)\right]
$$

where, $K\left(p_{i}, p j\right)=p_{i} \cdot p j \int_{0}^{1} d y \ln \left(p_{y}^{2} / \lambda^{2}\right) / p_{y}^{2}$ and $p_{y}=p_{i} y+p_{j}(1-y)$. In order to isolate the effect of the additional TPE contribution on the data, Blunden et al. [27] consider the following difference

$$
\Delta=\delta_{2 \gamma}-\delta_{I R}(M T)
$$

where the IR divergence part is independent of $\lambda$ cancel. The TPE corrections clearly play an important role in resolving the most of the form factor discrepancy. In terms of three complex form factors, $\tilde{F}_{1}, \tilde{F}_{2}$ and $\tilde{F}_{3}$, the generalized amplitude for elastic scattering is 
written as

$$
\mathscr{M}=-i \frac{e^{4}}{q^{2}} \bar{u}\left(p_{3}\right) \gamma_{\mu} u\left(p_{1}\right) \bar{u}\left(p_{4}\right)\left(\tilde{F}_{1} \gamma^{\mu}+\tilde{F}_{2} \frac{i \sigma_{\mu \nu} q_{\nu}}{2 M}+\tilde{F}_{3} \frac{\gamma \cdot K P^{\mu}}{M^{2}}\right) u\left(p_{2}\right)
$$

here $K=\left(p_{1}+p_{3}\right) / 2$ and $P=\left(p_{2}+p_{4}\right) / 2$. The functions $\tilde{F}_{i}$ are the functions of $Q^{2}$ and $\varepsilon$. The functions $\tilde{F_{1,2}}$ approach to the Dirac and Pauli form factors in $1 \gamma$ approximation, whereas the new form factor $\tilde{F}_{3}$ exists only at the $2 \gamma$ level and beyond,

$$
\begin{aligned}
\tilde{F_{1,2}}\left(Q^{2}, \varepsilon\right) & \rightarrow \tilde{F_{1,2}}\left(Q^{2}\right), \\
\tilde{F}_{3}\left(Q^{2}, \varepsilon\right) & \rightarrow 0 .
\end{aligned}
$$

In terms of Sachs form factors

$$
\begin{gathered}
\tilde{G_{E}}=G_{E}+\delta G_{E}, \\
\tilde{G_{M}}=G_{M}+\delta G_{M} .
\end{gathered}
$$

The reduced cross section up to order $\alpha^{2}$ can be written as,

$$
\tilde{\sigma}_{r}=G_{M}^{2}+\frac{\varepsilon}{\tau} G_{E}^{2}+2 G_{M}^{2} \mathscr{R} e\left(\frac{\delta G_{M}}{G_{M}}+\varepsilon Y_{2 \gamma}\right)+\frac{2 \varepsilon}{\tau} G_{E}^{2} R e\left(\frac{\delta G_{E}}{G_{E}}+\frac{G_{M}}{G_{E}} Y_{2 \gamma}\right)
$$

or as noted in [29] the reduce cross section can be written as,

$$
\tilde{\sigma}_{r}=G_{M}^{2}+2 G_{M} \operatorname{Re}\left(\delta \tilde{G_{M}}\right)+\frac{\epsilon}{\tau}\left[G_{E}^{2}+\frac{4 \tau^{2}}{M^{2}} \operatorname{Re}\left(\tilde{F}_{3}\right)\left(G_{M}+\frac{1}{\tau} G_{E}\right)+2 G_{E} \operatorname{Re}\left(\tilde{G_{M}}\right)\right]
$$

where, the $Y_{2 \gamma}$ and $\tilde{F}_{3}$ are related as

$$
Y_{2 \gamma}=\bar{\nu} \frac{\tilde{F}_{3}}{G_{M}}
$$


and

$$
\begin{aligned}
\bar{\nu} & \equiv \frac{K . P}{M^{2}}, \\
& =\sqrt{\tau(1+\tau)(1+\varepsilon) /(1-\varepsilon)} .
\end{aligned}
$$

Fig. 1.6 shows the TPE corrected Rosenbluth separation data in red. This plot indicates that after TPE correction to the Rosenbluth data, the discrepancy in $G_{E}$ to $G_{M}$ ratio between this data and the polarization transfer data has been significantly reduced.

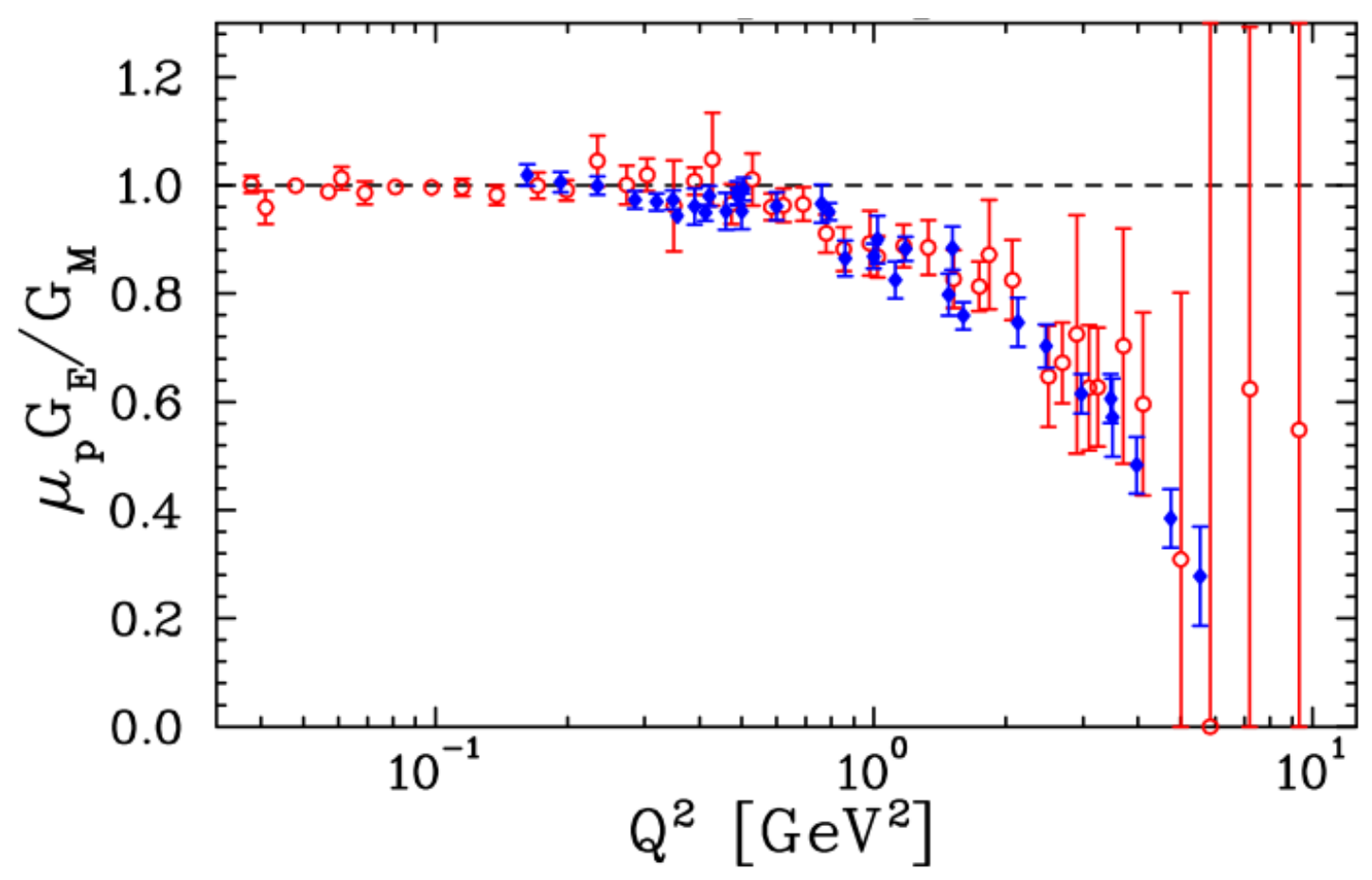

Figure 1.6. The ratio of proton electric and magnetic form factors measured using LT separation (red points) and polarization transfer (PT)(blue points). The LT points corrected for TPE are shown with filled square and filled circles.

\subsection{Global Fit}

As nucleon electromagnetic form factors are required for many calculations in nuclear physics, a simple parametrization that accurately represents the data over a wide range of 
$Q^{2}$ with reasonable behavior for both $Q^{2} \rightarrow 0$ as well as $Q^{2} \rightarrow \infty$ would be useful. To get reasonable behavior at low $Q^{2}$ the power series representation should involve only even powers of $Q$. On the other hand, at high $Q^{2}$ the dimensional scaling rules require $G_{M} \propto Q^{-4}$ . But the most common parametrizations violate both of the conditions. The simplest form of parametrization that fit to all cross section, polarization transfer, and beam-target asymmetry measurement as explained in Ref. [2] takes the following form:

$$
G_{E}, G_{M} / \mu_{p}=\frac{1+\sum_{i=1}^{n} a_{i} \tau^{i}}{1+\sum_{i=1}^{n+2} b_{i} \tau^{i}}
$$

Here, for $n=3$, there are eight fit parameters for each form factor, along with a normalization factor for each independent set of cross section measurements. The fit includes 529 cross section and 54 polarization transfer points and gives a reduced $\chi^{2}$ of 0.77 . The function in Eq. 1.58 is chosen so that it gives reasonable behavior in both low as well as high $Q^{2}$. The parameters $b_{i}$ are constrained to be positive, to avoid fits where both the numerator and denominator pass through zero at the same $Q^{2}$, giving narrow divergence in the fit.

For elastic cross sections, a separate parametrization without direct reference to TPE effects is provided in Ref. [2]. This will give a model-independent parameterization for the cross section without TPE corrections. This will parameterize full elastic reduced cross section including both Born and TPE corrections by:

$$
\sigma_{R}^{B o r n+T P E}=\tau F_{m}^{2}\left(Q^{2}, \varepsilon\right)+\varepsilon F_{e}^{2}\left(Q^{2}, \varepsilon\right)
$$

where, $F_{m}$ and $F_{e}$ are effective magnetic and electric form factors respectively, which consists of the effects of multiple photon exchange. In the Born approximation these two form factors 
are just the usual Sachs form factors:

$$
\begin{gathered}
F_{m}\left(Q^{2}, \varepsilon\right) \rightarrow G_{M}\left(Q^{2}\right), \\
F_{e}\left(Q^{2}, \varepsilon\right) \rightarrow G_{E}\left(Q^{2}\right) .
\end{gathered}
$$

The results of the fit for the cross section in Eq. 1.59 in terms of the effective form factors $F_{m}$ and $F_{e}$ are presented in the table 1.1.

Table 1.1

Parameters for the fit to the TPE-uncorrected cross section, using the parameterization for $F_{m}$ and $F_{e}$.

\begin{tabular}{|c|c|c|}
\hline Parameter & $F_{m} / \mu_{p}$ & $F_{e}$ \\
\hline$a_{1}$ & -2.151 & -1.651 \\
\hline$a_{2}$ & 4.261 & 1.287 \\
\hline$a_{3}$ & 0.159 & -0.185 \\
\hline$b_{1}$ & 8.647 & 9.531 \\
\hline$b_{2}$ & 0.001 & 0.591 \\
\hline$b_{3}$ & 5.245 & 0.000 \\
\hline$b_{4}$ & 82.817 & 0.000 \\
\hline$b_{5}$ & 14.191 & 4.994 \\
\hline
\end{tabular}

\subsection{Models of Form Factors}

\subsubsection{Vector Meson Dominance}

This is the earliest model of form factors introduced by Sakurai [30] to describe the electron-proton interaction through the coupling with vector mesons. The Feynman diagram for the VMD model is shown in Fig. 1.7. The VMD model differs from the other models by the inclusion of the $\rho, w$ and $\phi$ mesons along with higher excited states. This model successfully describes the form factors, photo-production, as well as absorption cross-sections and vector meson exchange for $\pi N$ and $N N$ scattering. This model is accurate for the low $Q^{2}$ domain 
but it fails to predict the masses and number of mesons. The nucleon form factor in terms of the meson-nucleon form factor in this model is expressed as

$$
F\left(Q^{2}\right)=\Sigma_{i} \frac{C_{\gamma V_{i}}}{Q^{2}+M_{V_{i}}^{2}} F_{V_{i}}\left(Q^{2}\right)
$$

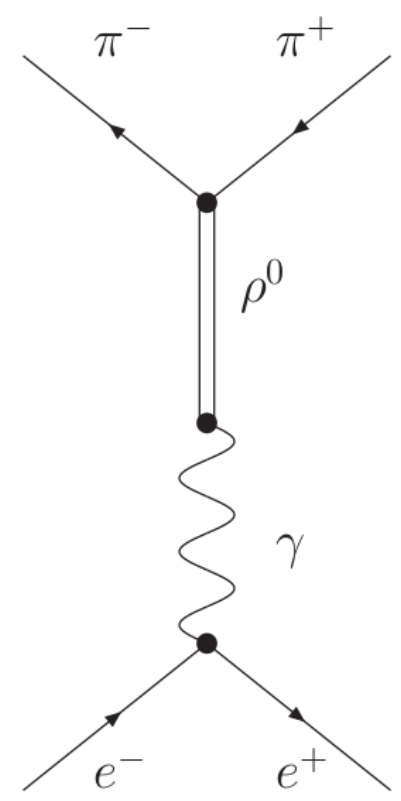

Figure 1.7. A Feynman diagram for a scattering process featuring VMD in which the virtual photon couples to the nucleon (shown as a black circle) through $\rho$ or $\omega$ mesons.

where $1 /\left(Q^{2}+M_{V_{i}}^{2}\right)$ is the meson propagator, $M_{V_{i}}$ are the meson masses, $F_{V_{i}}\left(Q^{2}\right)$ is the meson-nucleon form factor and $C_{\gamma} \mathrm{V}_{i}$ is the photon-meson coupling strength. This VMD model was very successful in describing the early low $Q^{2}$ form factor data. The early VMD fit performed by Iachello et.al [31] in 1973, predicted a linear decrease of the proton $G_{E}^{P} / G_{M}^{P}$ ratio. This was in agreement with the later results from the polarization transfer transfer measurements in JLab Hall A. However, the Iachello et. al. fit disagrees with the asymptotic behavior of the form factors at high $Q^{2}$ as predicted by pQCD.

The first work to merge the low $Q^{2}$ predictions of VMD with the high $Q^{2}$ behavior predicted by pQCD was done by Gari and Krümpelmann (GK) [32], using the extended 
version of VMD model where the photon-nucleon interaction has a purely photonic part in addition to the meson poles. This model assumes a complete decoupling of the nucleon with the OZI (Okubo-Zweig Iizuka) rule [33]. The isovector and isoscalar form factor in the GK model determined by the $\rho$ and $\omega$ mesons are given by

$$
\begin{aligned}
& F_{1}^{V}\left(Q^{2}\right)=F_{1}\left(Q^{2}\right)\left[\frac{g_{\rho}}{f_{\rho}} \frac{m_{\rho}^{2}}{m_{\rho}^{2}+Q^{2}}+\left(1-\frac{g_{\rho}}{f_{\rho}}\right)\right], \\
& F_{2}^{V}\left(Q^{2}\right)=F_{2}\left(Q^{2}\right)\left[\frac{g_{\rho} \kappa_{\rho}}{f_{\rho} \kappa_{V}} \frac{m_{\rho}^{2}}{m_{\rho}^{2}+Q^{2}}+\left(1-\frac{g_{\rho} \kappa_{\rho}}{f_{\rho} \kappa_{V}}\right)\right], \\
& F_{1}^{S}\left(Q^{2}\right)=F_{1}\left(Q^{2}\right)\left[\frac{g_{\omega}}{f_{\omega}} \frac{m_{\omega}^{2}}{m_{\omega}^{2}+Q^{2}}+\left(1-\frac{g_{\omega}}{f_{\omega}}\right)\right], \\
& F_{2}^{S}\left(Q^{2}\right)=F_{2}\left(Q^{2}\right)\left[\frac{g_{\omega} \kappa_{\omega}}{f_{\omega} \kappa_{V}} \frac{m_{\omega}^{2}}{m_{\omega}^{2}+Q^{2}}+\left(\frac{\kappa_{S}}{\kappa_{V}}-\frac{g_{\omega} \kappa_{\omega}}{f_{\omega} \kappa_{V}}\right)\right] .
\end{aligned}
$$

The hadronic form factors parameterized by GK are given by

$$
\begin{aligned}
& F_{1}\left(Q^{2}\right)=\frac{\Lambda_{1}^{2}}{\Lambda_{1}^{2}+\tilde{Q}^{2}} \frac{\Lambda_{2}^{2}}{\Lambda_{2}^{2}+\tilde{Q}^{2}}, \\
& F_{2}\left(Q^{2}\right)=\frac{\Lambda_{1}^{2}}{\Lambda_{1}^{2}+\tilde{Q}^{2}}\left[\frac{\Lambda_{2}^{2}}{\Lambda_{2}^{2}+\tilde{Q}^{2}}\right]^{2},
\end{aligned}
$$

where,

$$
\tilde{Q}^{2}=Q^{2} \frac{\log \left(\frac{\Lambda_{2}^{2}+Q^{2}}{\Lambda_{Q C D}^{2}}\right)}{\log \left(\frac{\Lambda_{2}^{2}}{\Lambda_{Q C D}^{2}}\right)},
$$

an $\Lambda_{1}$ is the meson scale cut off, $\Lambda_{2}$ is the quark-gluon scale cut off and $\Lambda_{Q C D}$ is the QCD scale cut off.

In a later work to fit electromagnetic form factors to the data considering the asymptotic behavior given by pQCD have been made by Lomon [34]. Lomon combines the GK model with the model of Höhler and Pietarinen (HP) [35]. In this model, the width of the $\rho$ meson is accounted for by an analytic approximation to the dispersion integral for the $\rho$ meson. 
Lomon also found that the inclusion of the $\rho$ width through the dispersion relation and the $\rho^{\prime}$ pole gave better results than the GK model. In addition to this, the best fits to $\mu_{p} G_{E} / G_{M}$ data of Bartel [3] and of Jones [17] using dispersion relations showed a slightly better $\chi^{2}$ than the GK model.

\subsubsection{Perturbative QCD (pQCD)}

The VMD model begins to break down for large $Q^{2}$, and because of its superposition of monopoles, it is not able to conform with the asymptotic behavior predicted by pQCD. pQCD is an approach from which the specific $Q^{2}$ dependence of the nucleon form factors can be obtained based on a factorization theorem. This theorem allows scale separation between the short and long distance motions of the quarks or gluons. The short distance motion corresponds to perturbative physics. The helicity conserving form factor of the nucleon in pQCD can be given by [36]

$$
F_{1}\left(Q^{2}\right) \propto \int\left[d x_{i}\right] \int\left[d y_{i}\right] \phi^{\dagger}\left(x_{i}, Q\right) T_{H}\left(x_{i}, y_{i}, Q\right) \phi\left(y_{i}, Q\right)
$$

where

$$
\left[d x_{i}\right]=d x_{1} d x_{2} d x_{3} \delta\left(1-\Sigma_{i} x_{i}\right)
$$

$x_{i}$ is the momentum fraction of the $i_{t h}$ valence quark, $\phi\left(x_{i}, Q\right)$ is the probability amplitude for the distribution of longitudinal distribution of the quarks in the initial state of the nucleon (y corresponds to the final-state quarks) and $T_{H}$ is the hard scattering amplitude. The series expansion of $\phi\left(x_{i}, Q\right)$ is given by

$$
\phi\left(x_{i}, Q\right)=x_{1} x_{2} x_{3} \sum_{n=0}^{\infty} a_{n} \phi_{n}\left(x_{i}\right)\left(\ln \frac{Q^{2}}{\Lambda^{2}}\right)^{-\gamma_{n}}
$$


where $a_{n}$ and $\gamma_{n}$ are some constants. The proton dirac form factor $F_{1}$ based on the Brodsky and Lepage [36] picture takes the following form:

$$
F_{1}\left(Q^{2}\right)=\frac{32 \pi^{2} \alpha_{s}^{2}\left(Q^{2}\right)}{9 Q^{4}} \sum_{n, m} b_{n, m}\left(\ln \frac{Q^{2}}{\Lambda^{2}}\right)^{-\gamma_{n}-\gamma_{m}}\left[1+\mathcal{O}\left(\alpha_{s}\left(Q^{2}\right), m / Q\right)\right]
$$

As the helicity-flip associated with Pauli form factor $F_{2}$ is suppressed at high $Q^{2}$, the leading term of the Eq. 1.72 can be replaced by $G_{M}$ :

$$
G_{M}\left(Q^{2}\right) \propto \frac{1}{Q^{4}}
$$

And the contribution of the Pauli form factor $F_{2}$ to the electric form factor $G_{E}$ is suppressed by a factor of $Q^{2}$ i.e.

$$
\begin{aligned}
G_{E}\left(Q^{2}\right) & \propto \frac{1}{Q^{6}} \\
& \sim \frac{G_{M}}{Q^{2}} .
\end{aligned}
$$

This prediction is a natural consequence of hadron helicity conservation. The hadron helicity conservation arises from the vector coupling nature of the quark-gluon interaction, the quark helicity conservation at high energies, and the neglect of the non-zero quark orbital angular momentum state in the nucleon. The scaling result predicts that the ratio: $G_{E} / G_{M}$ become constant at high $Q^{2}$. This results were confirmed in a short-distance pQCD analysis made by Brodsky and Lepage [36]. Fig. 1.8 shows the magnetic form factor of the proton times $Q^{4}$ plotted as a function of $Q^{2}$. The asymptotic behaviour appears to start at approximately $5(\mathrm{GeV} / \mathrm{C})^{2}$ with slow variations at high $Q^{2}$. 


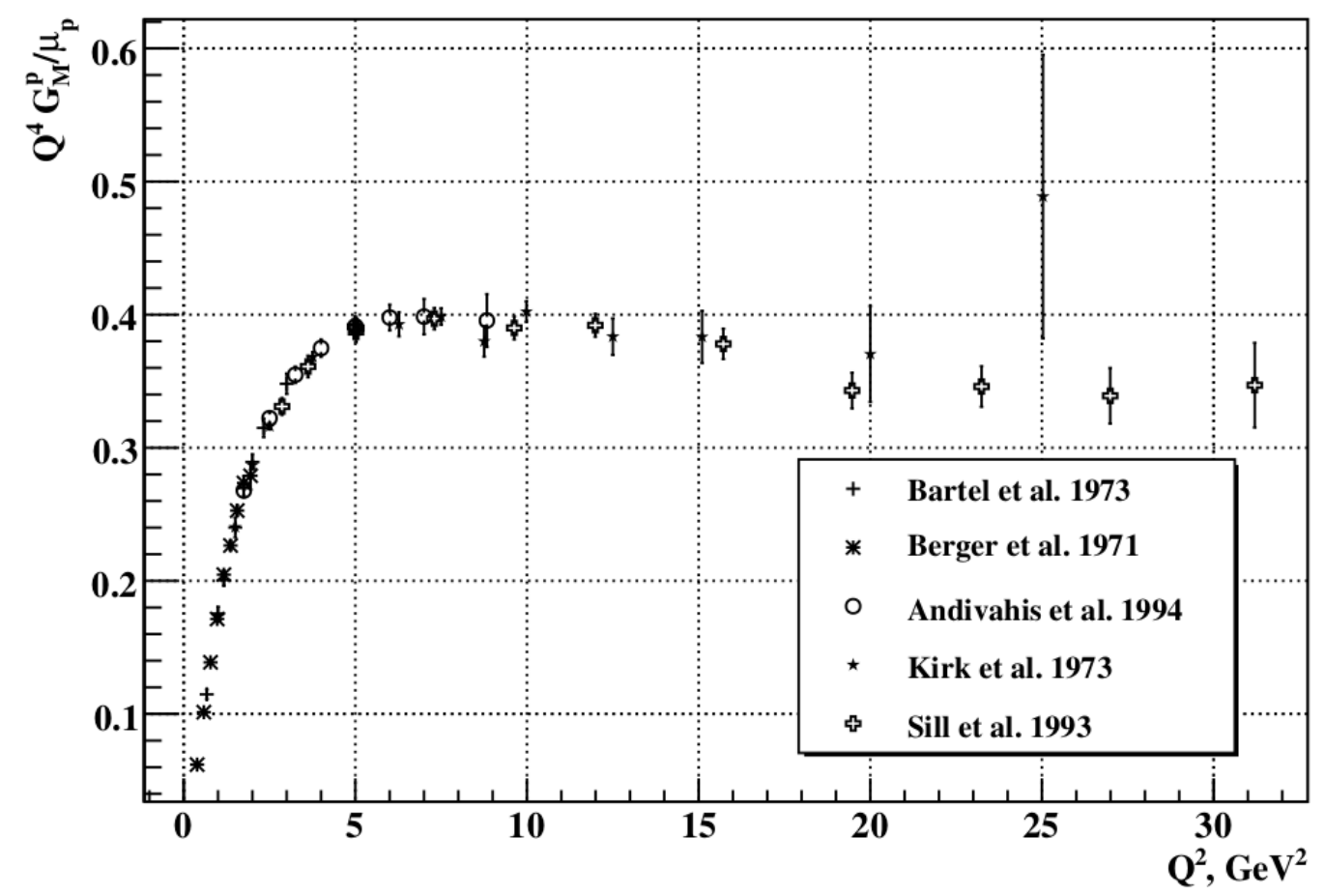

Figure 1.8. Plot of $Q^{4} G_{M}^{p} / \mu_{p}$ showing the asymptotic behavior of the proton magnetic form factor. The data are: Bartel et al. is from Ref. [3], Berger et al. is from Ref. [4], Andivahis et al. is from Ref. [5], Kirk et al. Ref. [6], Sill et al. is from Ref. [7].

\subsection{Existing Magnetic Form Factor Data}

This section will present an overview of previous experimental data on the proton magnetic form factor. Fig. 1.9 shows the data for $G_{M}^{p}$ obtained from the Rosenbluth separation method, where $G_{M}^{P}$ is normalized to the dipole form factor. It is evident from the plot of $G_{M}^{p} / \mu_{P} G_{D}$ vs $Q^{2}$ that most of the data for $G_{M}^{p}$ are for $Q^{2}<8 \mathrm{GeV}^{2}$. The best data set above $8 \mathrm{GeV}^{2}$ is the Sill measurement from SLAC [7] with cross section uncertainties varying from $4 \%$ at $Q^{2}=5 \mathrm{GeV}^{2}$, to $8 \%$ for $Q^{2} \approx 20 \mathrm{GeV}^{2}$, without including an overall normalization uncertainty of $3 \%$. The Sill data extracted $G_{M}^{p}$ assuming the scaling behavior of the form factors i.e. $G_{E}^{p}=G_{E}^{p} / \mu_{p}$ and that $G_{E}^{p}$ contribute $6 \%(2 \%)$ to the cross section at $Q^{2}=5 \mathrm{GeV}^{2}\left(20 \mathrm{GeV}^{2}\right)$. The recent polarization transfer measurements [19] show that $G_{E}^{p}$ is significantly smaller for the $Q^{2}$ where the ratio $G_{E}^{p} / G_{M}^{p}$ has been measured. This method 


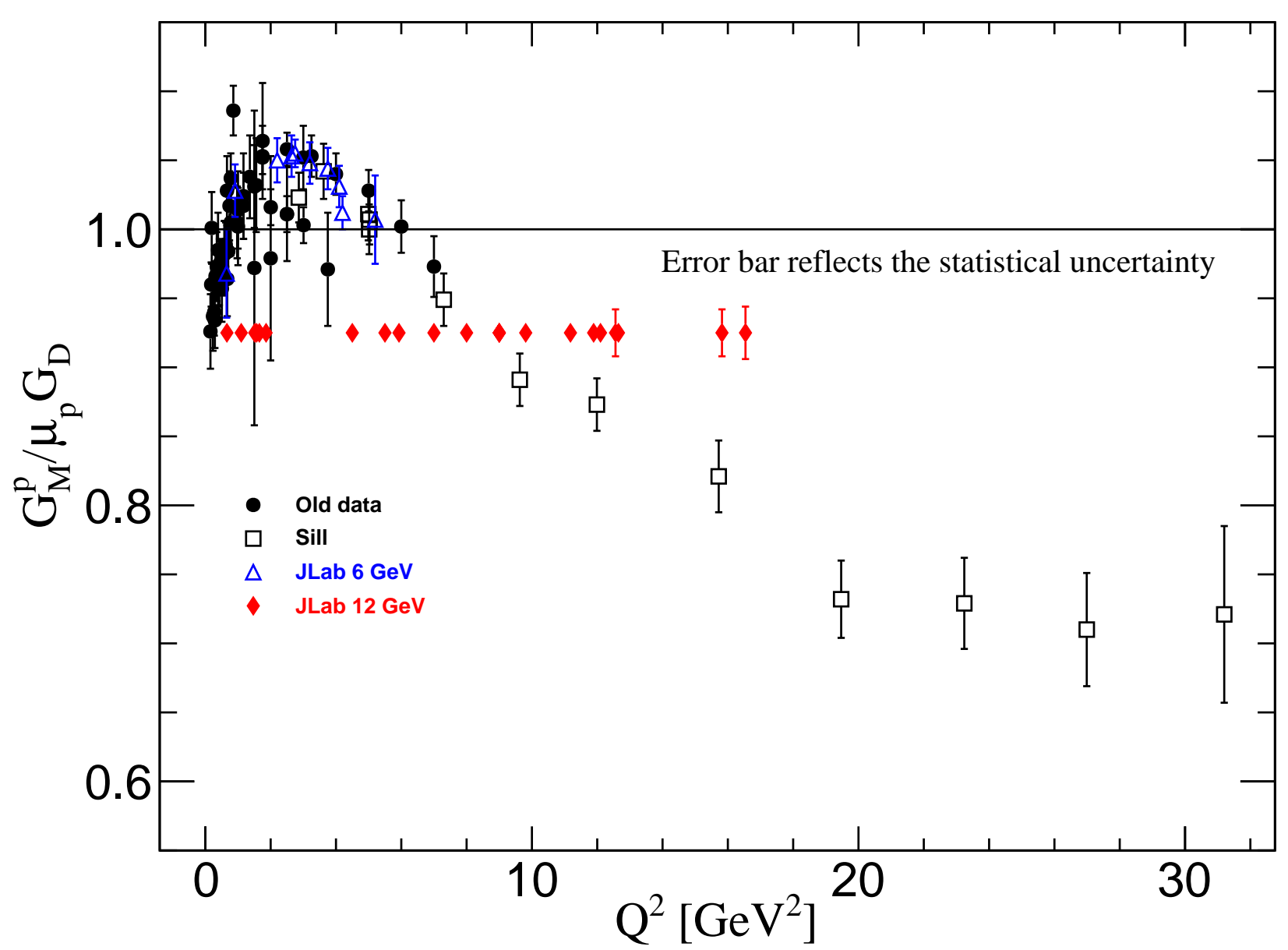

Figure 1.9. Proton magnetic form factor world data normalized by the dipole form factor from unpolarized measurements. The old data are from Refs. [3-7], Jlab $6 \mathrm{GeV}$ data refers to the Christy et al. Ref. [8] and JLab $12 \mathrm{GeV}$ refers to the data this experiment collected. Uncertainties shown do not include the effect of the normalization uncertainty, which is $3 \%$ on the Sill et al.

also shows that the true contribution from $G_{E}^{p}$ will be zero near $Q^{2} \approx 6-7 \mathrm{GeV}^{2}$, where $G_{E}^{p} / G_{M}^{p}$ becomes negative and increases in magnitude with $Q^{2}$. Also, $G_{E}^{p}$ contribution has found to be increase by $4 \%$ between $Q^{2}=5$ and $15 \mathrm{GeV}^{2}$. This showed a significantly modified $Q^{2}$ dependence for $G_{M}^{p}$. Another problem in the analysis of all previous measurements is that they all neglected the contribution of TPE. This effect yields a major correction to the extracted value of $G_{M}^{p}$ as discussed in earlier section. 


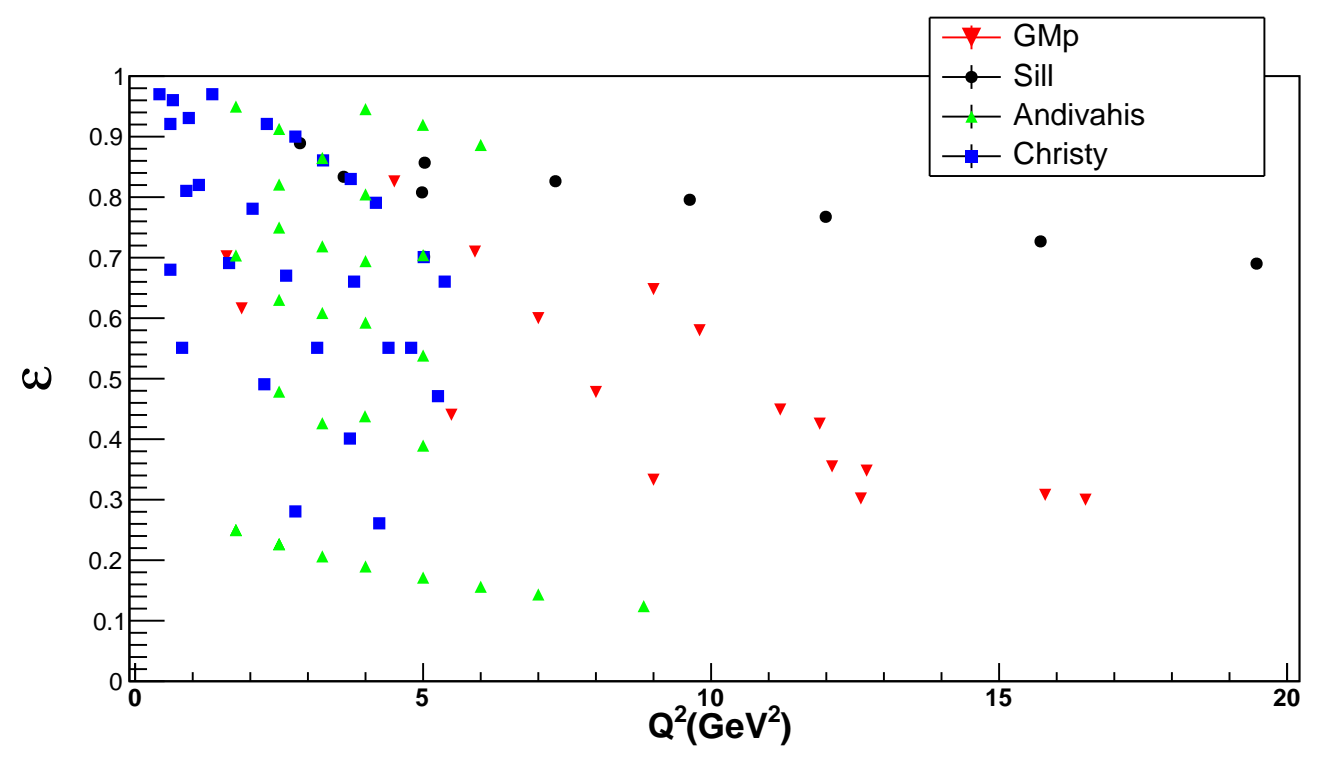

Figure 1.10. Kinematic coverage of GMp data (Red) compared to SLAC data [7] (black) in $\varepsilon$ vs $\mathrm{Q}^{2}$ plot.

Fig. 1.10 shows $\varepsilon$ vs $Q^{2}$ coverage for several ep elastic experiments at $Q^{2} \geq 0.5 \mathrm{GeV}^{2}$. This plot shows that the GMp data are at lower $\varepsilon$ then SLAC data. This implies that the measured cross sections will have a smaller contribution from the electric form factor allowing a higher precision of extraction of the magnetic form factor. 


\section{CHAPTER 2 \\ EXPERIMENTAL SETUP}

\section{$2.1 \quad$ Overview}

Experiment E12-07-108, was conducted in experimental Hall A at the Thomas Jefferson National Accelerator Facility from Spring 2015 to Fall 2016. The purpose of this experiment was to precisely measure the elastic electron-proton cross-section in a wide range of $Q^{2}$ with a total uncertainty better than $2 \%$. The experiment utilized the continuous electron beam of Continuous Electron Beam Accelerator Facility (CEBAF) and a $15 \mathrm{~cm}$ long liquid Hydrogen target. The scattered electrons were detected in the High Resolution Spectrometers (HRS), which are standard equipment in Hall A. The production data were acquired in three separate run periods with beam energies of $2.2,4.5,6.4,8.5$ and $10.6 \mathrm{GeV}$ and electron scattering angles ranging from $17^{\circ}$ to $55.9^{\circ}$. A total of twenty one $Q^{2}$ points were collected during entire experimental run period, with the kinematic coverage is shown in Fig. 1.9. In this chapter, the experimental setup for the experiment will be discussed and will include a brief description of the continuous electron beam accelerator along with the beamline hardware, target and spectrometers in experimental Hall A. This chapter will conclude with an overview of the electronics and data acquisition systems.

\subsection{Thomas Jefferson National Accelerator Facility}

The CEBAF accelerator at Jefferson Lab provides continuous wave (cw) electron beam with currents up to $80 \mu A$ [37] and energies ranging from 2.2 to $10.6 \mathrm{GeV}$ during the running of this experiment. The layout of the CEBAF accelerator is shown in Fig. 2.1. The source of electrons at the injector is a $100 \mathrm{kV}$ photocathode gun, with the electrons originating from the photocathode gun then accelerated to $50 \mathrm{MeV}$ and then injected into the north linac 
where it is accelerated up to $600 \mathrm{MeV}$. At the end of the north linac, $180^{\circ}$ bending arcs with a radius of 80 meters join the north linac to the identical and antiparallel superconducting south linac forming a recirculating beamline. Each linac contains 25 cryomodules. The beam through each arc is focused and steered using quadrupole and dipole magnets located inside each arc.

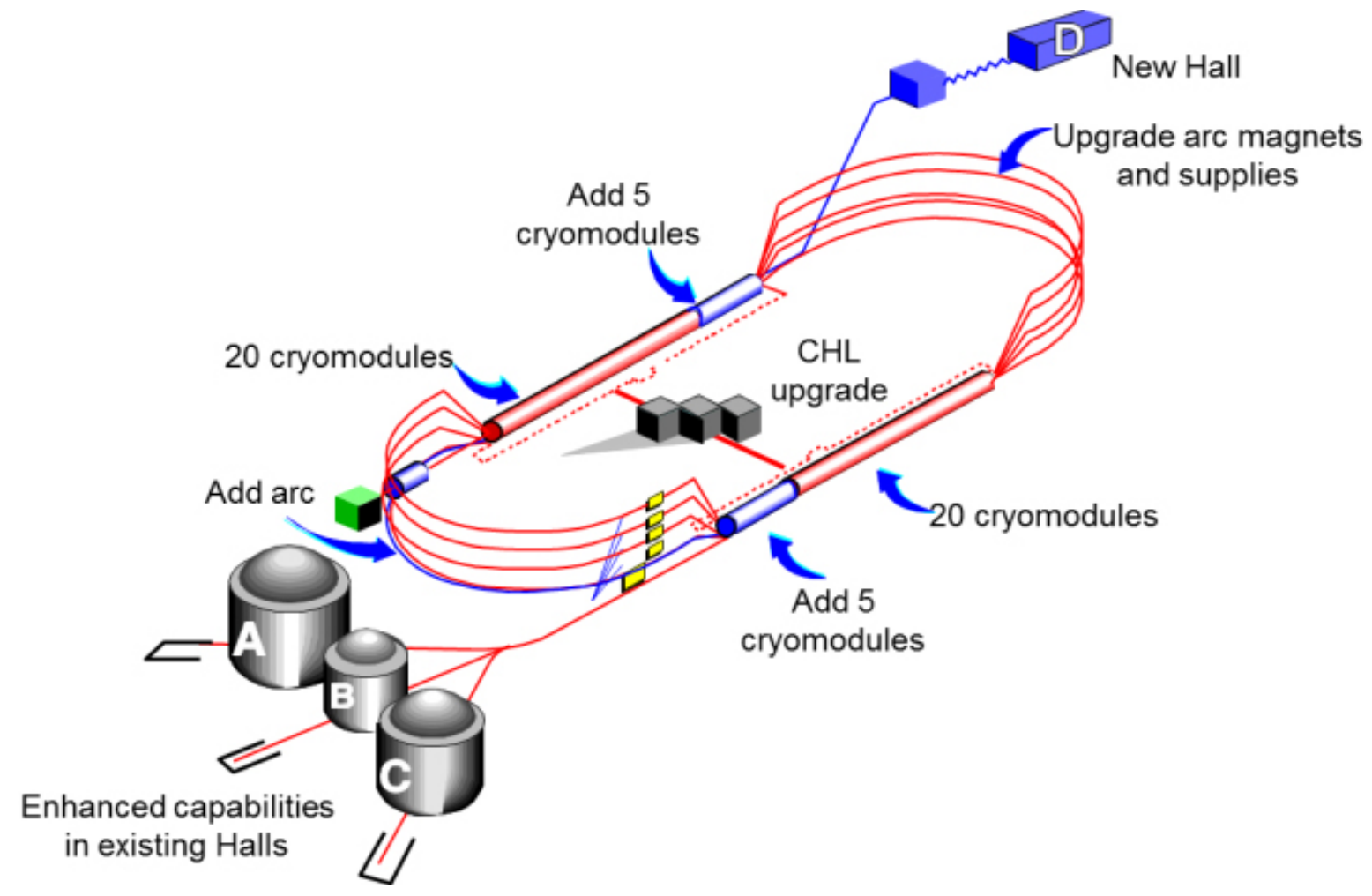

Figure 2.1. Layout of the CEBAF accelerator facility.

At the end of the south linac are three underground experimental halls into which the beam can be diverted and fed at different levels of energy simultaneously through the beam switchyard. The energy of the beam depends on the total number of passes. There are five different arcs for circulation on the east of the machine and four arcs on the west end. 


\subsection{Hall A Overview}

Experimental Hall A is the largest hall among the four Halls. The layout of Hall A is shown in Fig. 2.2. The beam is steered into Hall A through the Hall A arc, which is equipped with a variety of magnets to bend the beam into Hall and provide a beam energy measurement. The Hall A beamline instrumentation as shown in Fig. 2.3 consist of superharps, beam current monitors (BCMs), raster magnets and beam position monitors (BPMs). After passing through the beamline, the beam reaches to the target, where the nuclear interactions of interest take place. Hall A is built underground in a circular shape with a diameter of $53 \mathrm{~m}$ and a height of $17 \mathrm{~m}$ [10]. The standard Hall A apparatus consists of two high resolution spectrometers (HRSs): the left HRS (LHRS) and the right HRS (RHRS). Both were utilized in this experiment. A detailed discussion of the beamline and spectrometers will be given in later in this chapter.

\subsection{Hall A Beamline Instrumentation}

Fig. 2.3 shows the Hall A beamline. The beamline instruments used to determine the properties of the beam, such as beam energy, beam charge, position and direction are shown schematically.

\subsubsection{The Hall A Arc and Beam Energy Measurement}

The Hall A arc contains eight identical dipole magnets and two pairs of wire scanners (super harps) located at the entrance and exit. The wire scanners are used to measure the bend angle. During operation a ninth reference dipole is used, which is both identical and powered in series with the other dipoles. When the dipole string is energized, a mapping device measures the field integral of the ninth dipole, which can be used with the nominal bend angle of the arc to determine the energy of the beam. This method of beam energy

measurement is called the arc energy method. The basic concept of the beam energy mea- 


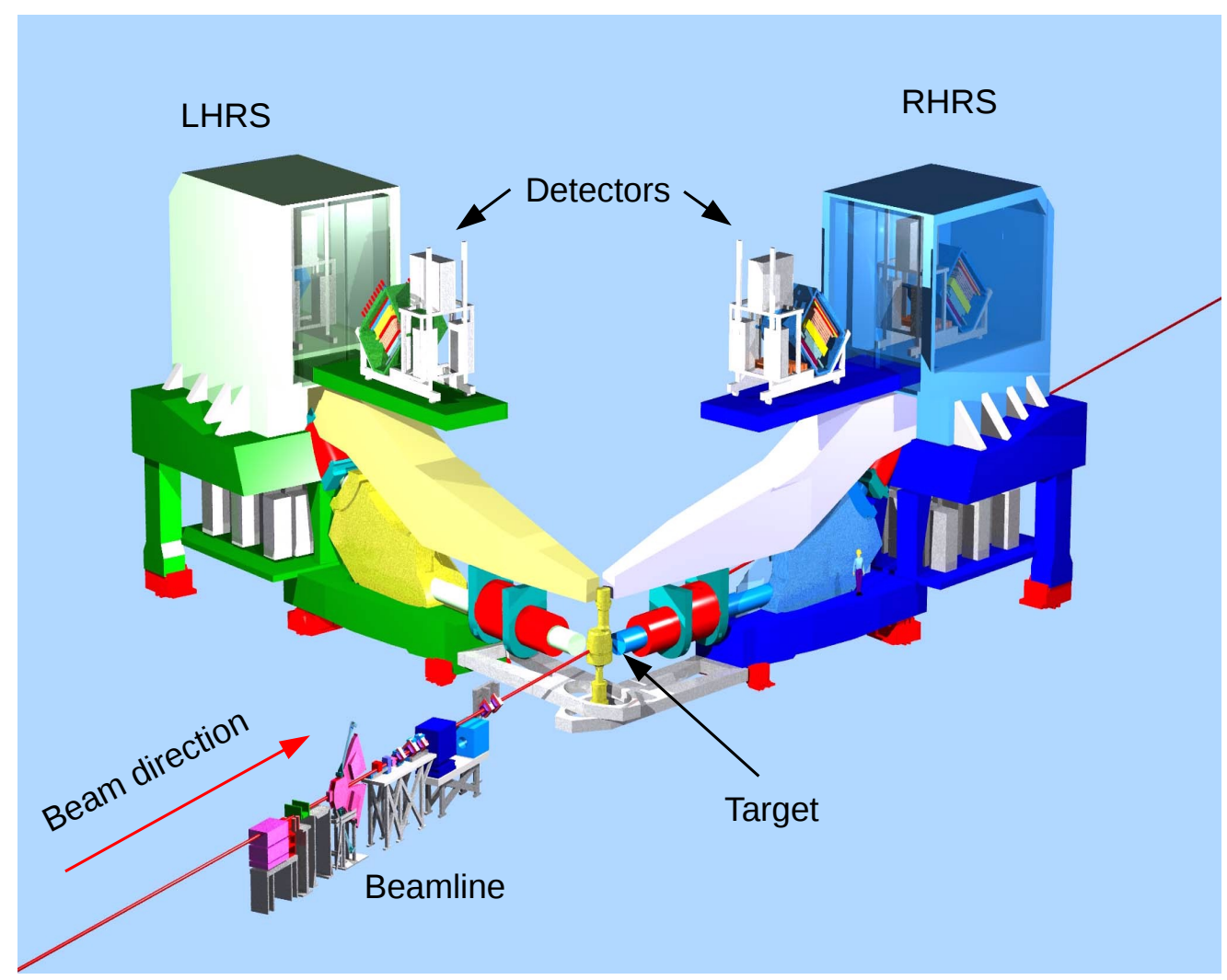

Figure 2.2. Schematic view of experimental apparatus consisting two high resolution spectrometers (HRS) on either side of the beam line. The front view of the spectrometer is shown in Fig. 2.11. The scattered electrons are detected in two HRS's: LHRS and RHRS.

surement is as follows: when an electron moves in a circular trajectory with velocity $\vec{v}$ in the region of field $\vec{B}$, it experiences a force (Lorentz force) and will be deflected by a known angle. The magnetic force on the electron is given by:

$$
\frac{d \vec{p}}{d t}=\frac{e}{c} \vec{v} \times \vec{B}
$$




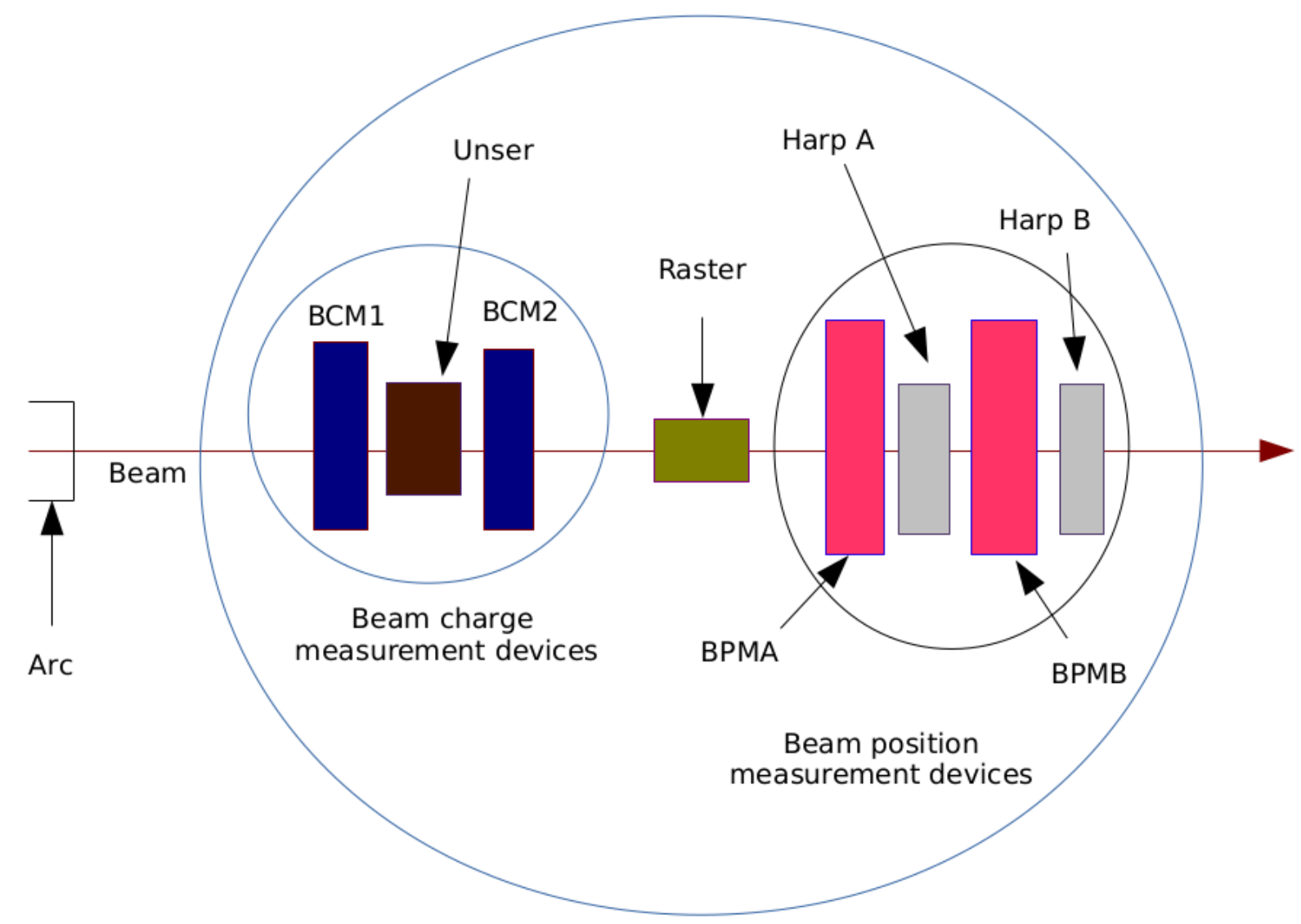

Figure 2.3. Layout of Hall A Beamline components.

So, if the magnetic field and the electron trajectory are known the electron energy can be determined as:

$$
E=\frac{k}{\theta} \int \vec{B} \cdot \overrightarrow{d l}
$$

where, $K=0.299792 \mathrm{GeV} \operatorname{rad} \mathrm{T}^{-1} m^{-1} / c$ and $\mathrm{B}$ is the field strength. Here, the beam energy can be calculated by measuring its bend angle through a well determined magnetic field. The arc energy method provides an absolute measurement to an accuracy of about $5 \times 10^{-4} E$ for the first pass beam for the GMp experiment. The beam energy for this experiment was also monitored by the "Tiefenbach" method, which uses Beam Position Monitors (BPMs) to monitor the bend angle, and therefore, the energy fluctuations. 


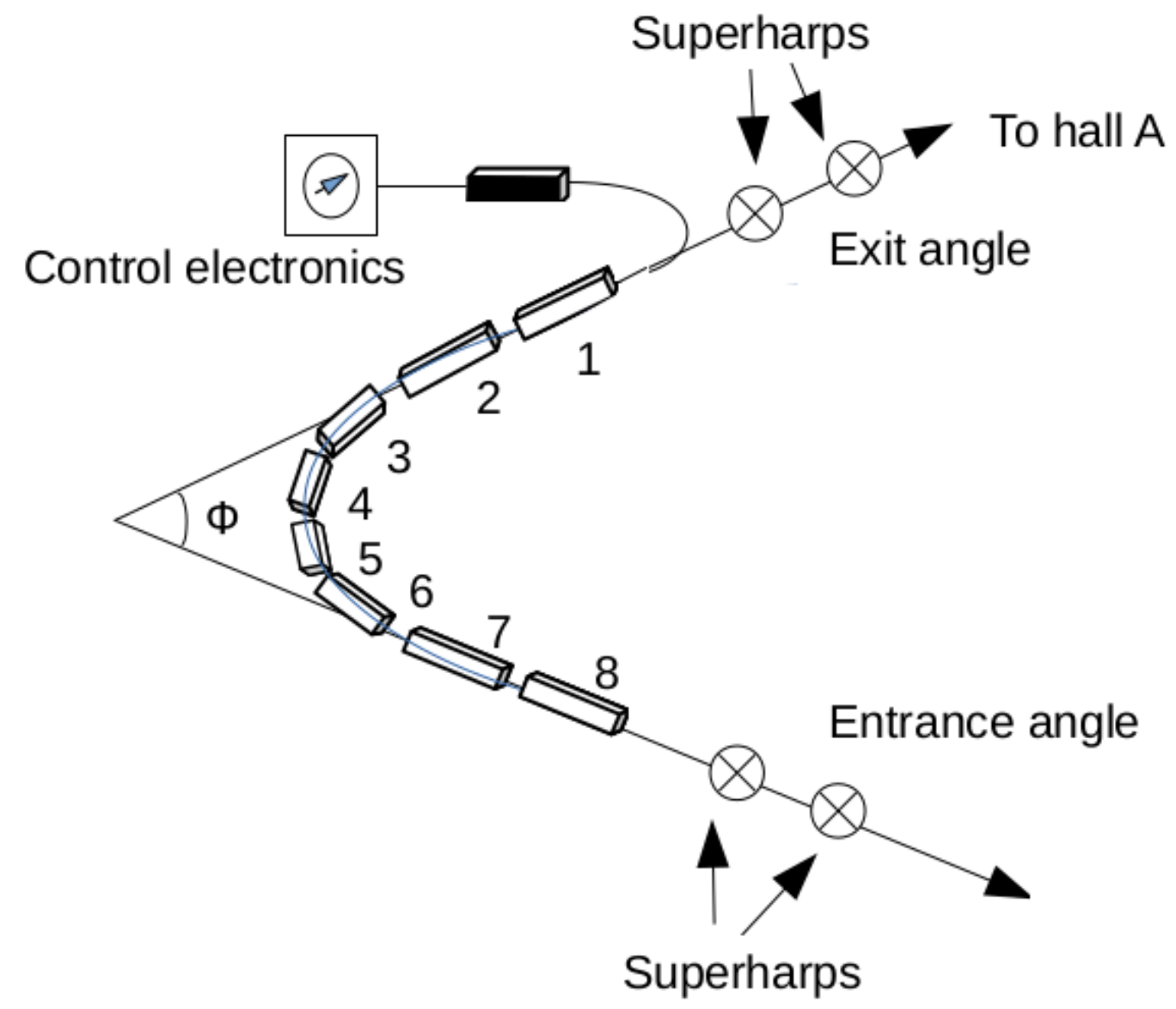

Figure 2.4. Hall A arc schematic.

\subsubsection{Beam Position Measurement}

The position and direction of the beam at the target is determined using two Beam Position Monitors (BPMs) located $7.524 \mathrm{~m}$ (BPMA) and $1.286 \mathrm{~m}$ (BPMB) upstream of the target, providing non-destructive measurement $[38,39]$. Each BPM consists of a cylindrical cavity with a set of four wires $\left(X_{+}, X_{-}, Y_{+}\right.$and $\left.Y_{-}\right)$, aligned parallel to the beam direction. The position measurement method is based on comparing the signals induced by the beam passing between each pair of antennas. By using the recorded signals from two such pairs, beam coordinates at the center of the BPM module can be reconstructed. By combining the information from both modules, a relative position of the beam projected to the target can be 
determined to within $100 \mu \mathrm{m}$ for currents above $1 \mu \mathrm{A}$. The effective offset corrected (pedestal subtracted) relative beam position $X^{\prime}$ and $Y^{\prime}$ along the axis of the wires is calculated by a difference over sum technique:

$$
X^{\prime}=k\left(\frac{X_{+}-X_{-}}{X_{+}+X_{-}}\right), \quad Y^{\prime}=k\left(\frac{Y_{+}-Y_{-}}{Y_{+}+Y_{-}}\right)
$$

The BPMs are calibrated using a set of Harps located adjacent to the BPMs . This in-

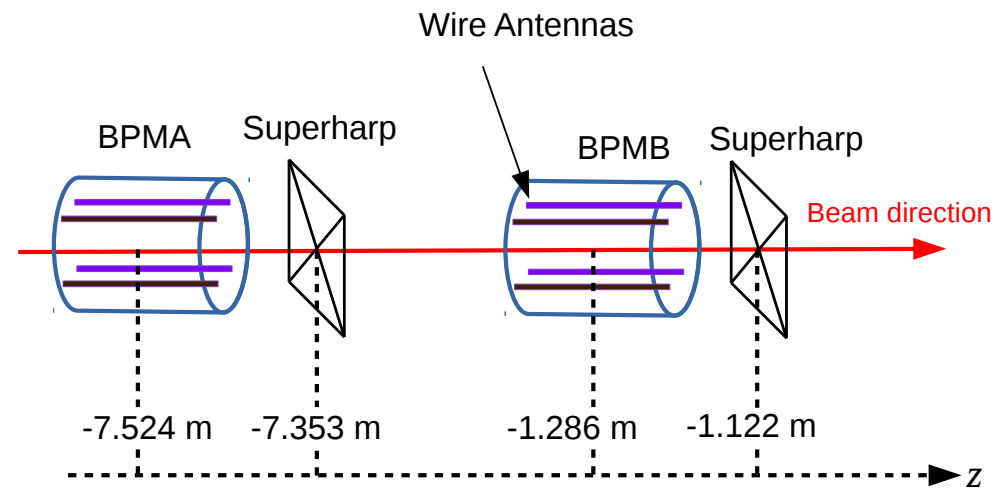

Positions in Hall A coordinate system

Figure 2.5. Schematic of beam position measurement.

vasive calibration procedure is called a "bull's eye scan". The Harps provide an invasive measurement of the beam position and consist of three wires oriented vertically at $\pm 45^{\circ}$. A schematic of the Harps is shown in Fig. 2.6. They operate by moving differently-oriented wires across a low current beam and reading out the induced wire signals. The Harps are routinely surveyed with respect to Hall Coordinate system. In Fig. 2.6, $\mathrm{X}_{\mathrm{H}}$ and $\mathrm{Y}_{\mathrm{H}}$ are the hall coordinates whereas, $\mathrm{X}_{ \pm}$and $\mathrm{Y}_{ \pm}$are the $\mathrm{BPM}$ coordinates, which are rotated by $45^{\circ}$ with respect to hall coordinate system. 

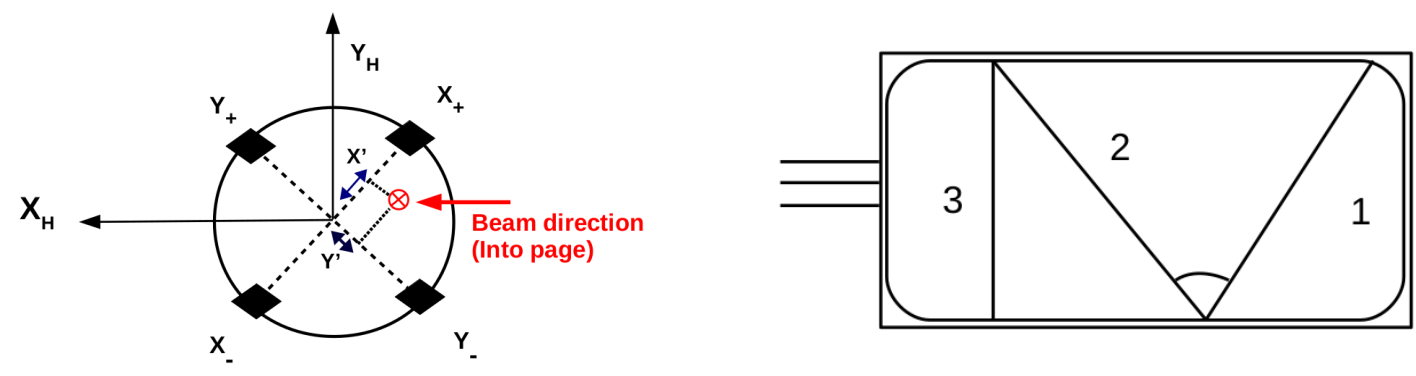

Figure 2.6. BPM coordinates system are shown by dotted lines (left) and a Harp (right) consisting three thin wires. The BPM coordinate system is rotated by $45^{\circ}$ with respect to nominal hall coordinate system.

\subsubsection{Beam Rastering}

The electron beam delivered to Hall A is CW (continuous wave) and with an rms of $\sim 200 \mu \mathrm{m}$. The raster is a pair of horizontal (X) and vertical (Y) air-core dipoles located 23 $m$ upstream of the target. Beam rastering was used during the experiments to reduce the effects of LH2 boiling and possible damage to the target can. The rastering reduces local heating of any component of the target or dump due to the high power density of energy deposition in the target. However, effects of local reduction of the LH2 target density due to the heat deposition of the beam into the LH2 are observed even with the use of the rastering system. To account for this effect, a study of target boiling was performed during both experiments. A correction factor to the effective target length was also applied to account for contraction during cool-down.

If the raster is off, the beam position can be determined using the two BPMs. If the raster is on, there is a phase lag between the real position and the position recorded by BPM. So the raster current that is recorded for each event will need to be used. Fig. 2.7 shows the raster pattern used for this experiment. The BPMs are not fast enough to get the positions event by event. The raster raw current is used to indirectly determine beam position [40]. The calibration of raster is done by comparing the raster raw current with the average beam position from the BPM. The Raster correction is necessary to get rid of any 


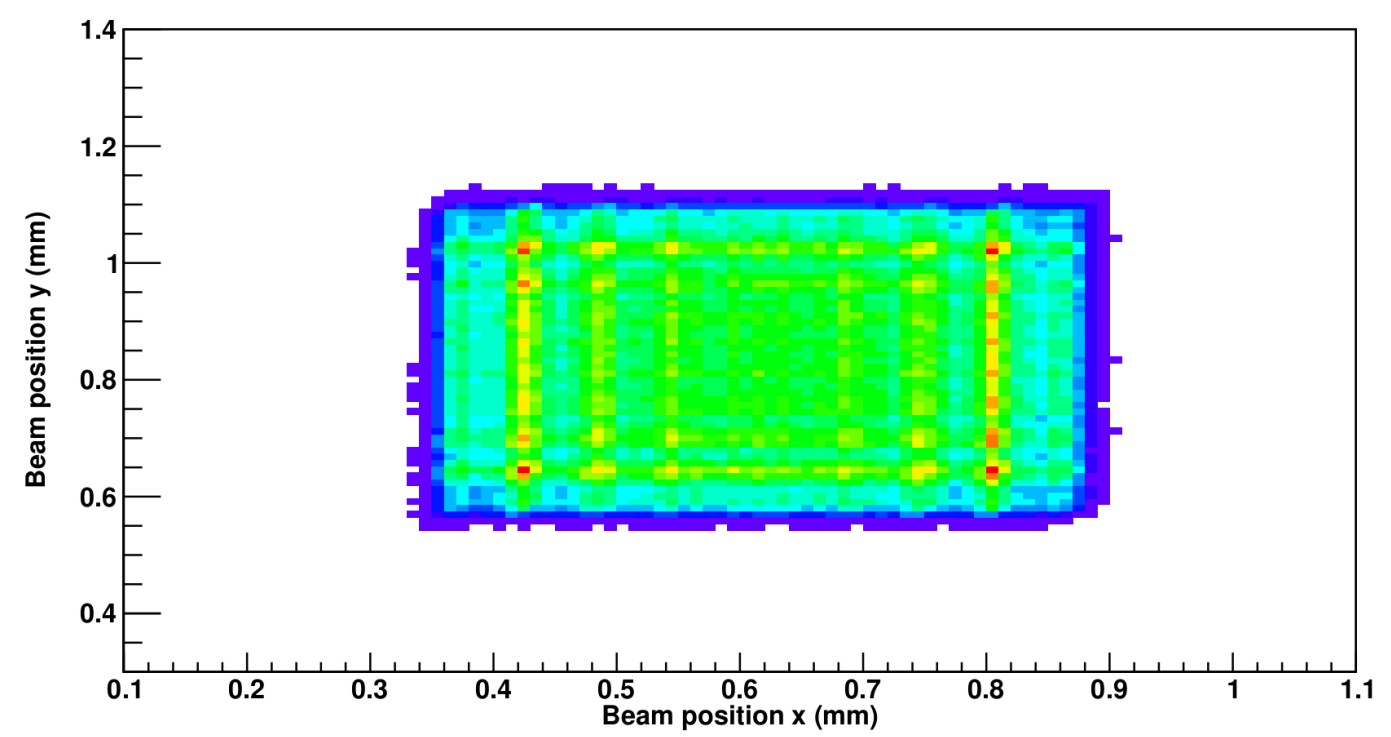

Figure 2.7. The density of the events vs beam position projected to the target from BPM.

deviation in beam position offset. If we do not account for raster correction, the variables at the target will be incorrectly reconstructed. This will impact the reconstructed momentum of the detected particles, as a deviation in the Hall vertical direction $\left(\Delta x_{t g}\right)$ creates a deviation in the focal plane $\left(\Delta x_{f p}\right)$ thereby affecting $\Delta \delta$.

\subsubsection{Beam Current Measurement}

The complete beam current monitoring system consisting two beam current monitors $(\mathrm{BCMs})$ and an Unser monitor is located $25 \mathrm{~m}$ upstream from the target, as illustrated in Fig. 2.8. The BCMs are designed for stable, low noise, non invasive beam current measurements. The Unser monitor is a parametric current transformer (PCT) which is simply a toroidal transformer with the beam acting as a single turn. The Unser monitor is calibrated by passing a precisely known current through an internal wire and provides an absolute reference. However, the Unser offset is noisy and the time average offset drifts in unpredictable ways. By measuring a series of currents over a short period of time, the absolute current can 
be determined by the Unser monitor, then transferred to the BCM's. A BCM consists of a

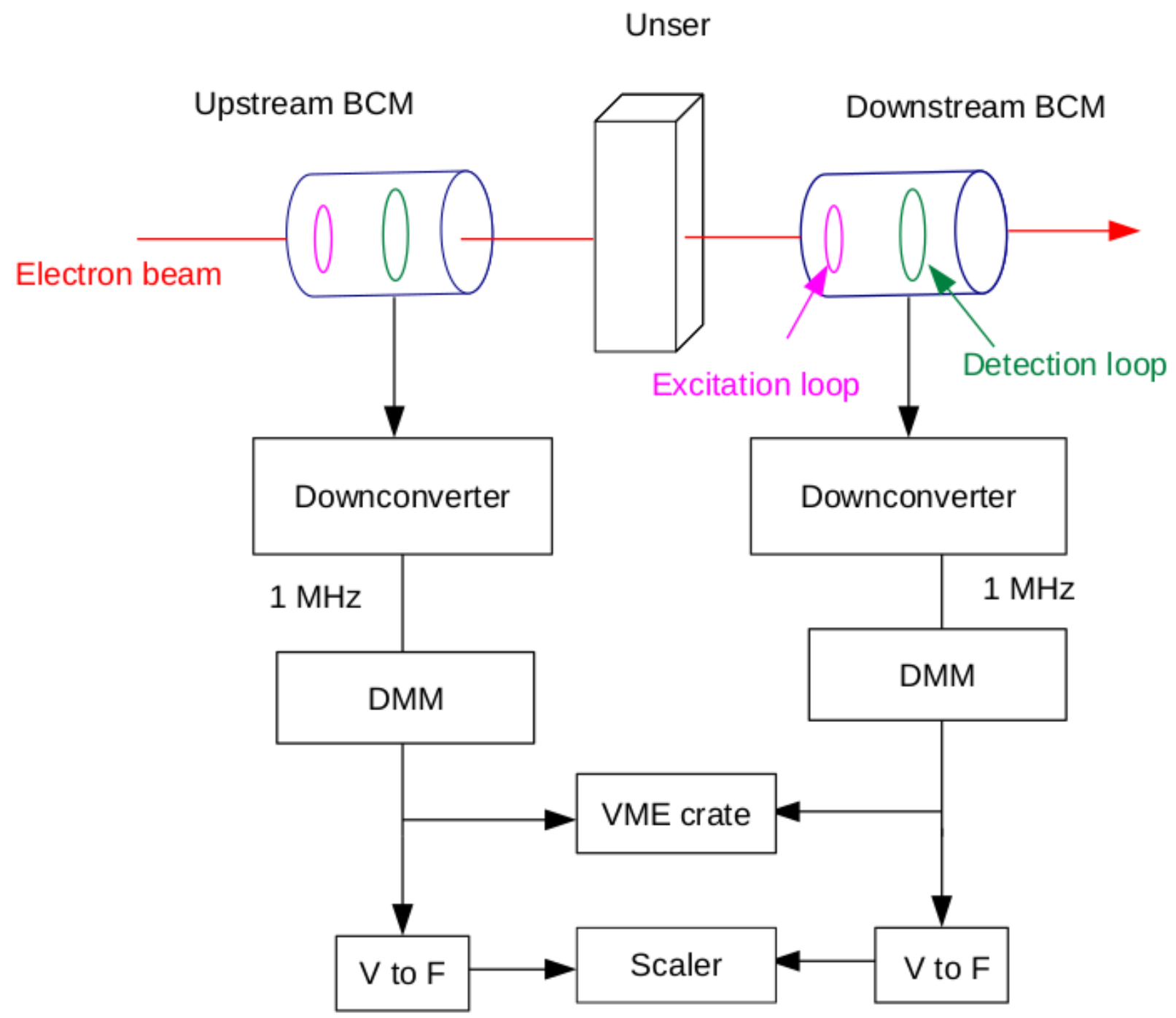

Figure 2.8. Schematic of HallA beam current measurement.

stainless steel cylindrical cavity, $15.48 \mathrm{~cm}$ in diameter and $15.24 \mathrm{~cm}$ in length [10], with axis coinciding with the nominal beam position. Resonant frequencies of the cavities are tuned to the frequency of the beam. Inside each cavity there are two loop antenna, one of which provides an output signal proportional to the beam current. The RF output signal from the cavity is down converted to a $1 \mathrm{MHz}$ signal and fed to an RMS to DC converter board. After this conversion two output signals are produced: 
- Signal 1 is sent to a high precision digital AC converter which produces a digital output as the RMS value of the input signal averaged over a one second period. The conversion of the voltage to current for each cavity has to be determined in a calibration procedure.

- Signal 2 is split into three signals, with each sent to amplifiers with relative gains of $\times 1, \times 3, \times 10$. The $\times 1$ signal are linear for beam currents from $10-200 \mu \mathrm{A}$. The $\times 3$ gain can be used for beam currents up to about $60 \mu \mathrm{A}$. The 10 amplified signal is used to measure the currents upto about $30 \mu \mathrm{A}$. Each amplified signal is then sent to an RMS to DC converter and the resulting DC voltage level is sent to a voltage to frequency (V-to-F) converter, with the output from (V-to-F) is fed to a scaler. The number of counts measured by the scaler is proportional to the integrated charge. The constant of proportionality between the $\mathrm{V}$ to $\mathrm{F}$ frequency and current has to be determined from calibration.

In addition, there are two new digital receivers: unew and dnew for which a DAQ output is first converted to an analog signal and then fed to V-F. However, these receivers were not used in the analysis because their gain changed from one period to another by an order of magnitude.

\subsection{Scattering Chamber}

As shown in Fig. 2.9 the scattering chamber used in this experiment consists of three main sections. The bottom section was fixed on the pivot of the hall and made of stainless steel. This section also contains several viewports through which the targets could be visually inspected and several ports for pulling vacuum. The middle section is an aluminum cylinder located at the beam height of $15.2 \mathrm{~cm}$ with vertical cutouts on each of the beam sides over the full angular range of $12.5^{\circ}<\theta<167.5^{\circ}$. This section has beam entrance and exit ports which are vacuum coupled to the electron beamline in order to prevent the beam from interacting with materials other than the target. The scattered particles exit the 


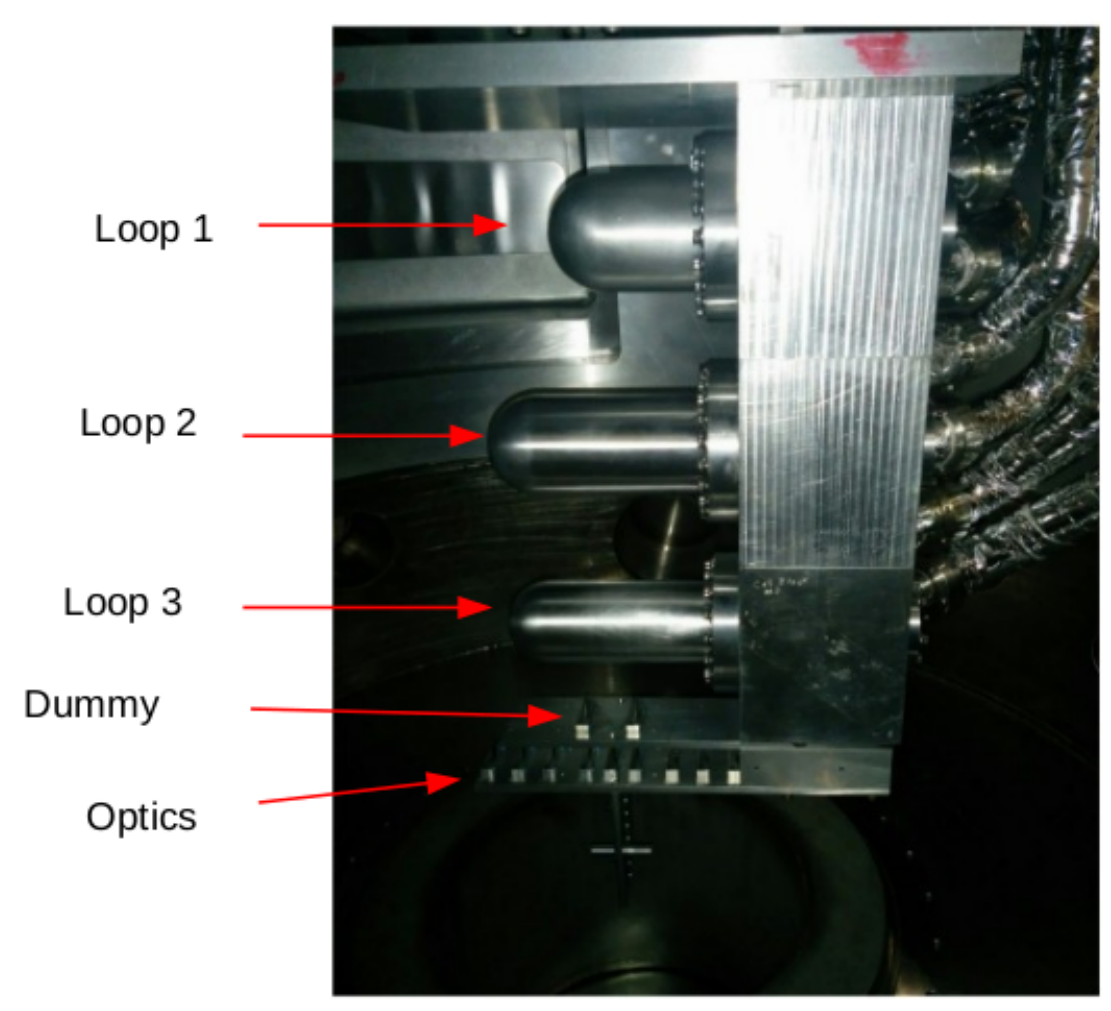

Figure 2.9. Hall A target scattering chamber. Cryotarget loops on top and solid targets on the bottom are visible [9].

chamber to the spectrometer through aluminum exit windows. The third section of the chamber contained space for the cryogenic target plumbing and instrumentation related to its coolant. All three sections of the chamber were maintained under vacuum. More details on the description of the scattering chamber used in this experiment can be found in Ref. [41].

A schematic of the target lifter GUI showing the components on the target ladder used in this experiment is shown in Fig. 2.10. The ladder contains subsystems for cooling, gas handling, temperature and pressure monitoring and target control and motion. There are three cryogenic target loops. The loop 1 target is a $4 \mathrm{~cm}$ long target holding helium gas. We could not use this target as we did not have time to cool the helium gas. Loop 2 is a 15 cm cell containing liquid hydrogen (LH2), and Loop 3 is a $15 \mathrm{~cm}$ cell containing LH2. All 


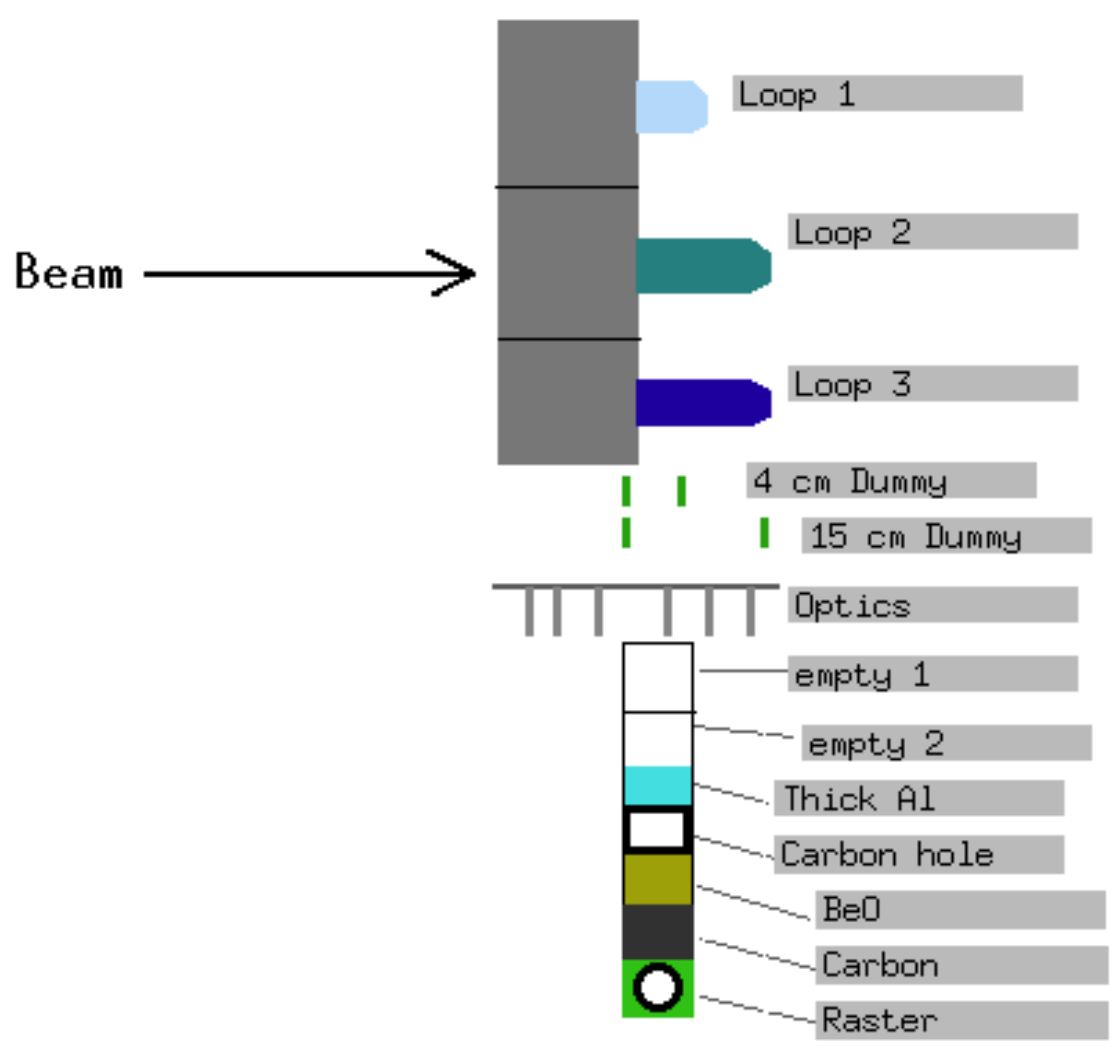

Figure 2.10. Target lifter GUI (Graphic User Interface) indicating positions of various targets.

three loops are cylindrical aluminum cells ending with a hemispherical tip. Fans are used to circulate the liquid or the gas through each loop. The operating temperature and pressure of LH2 target are regulated to $19 \mathrm{~K}$ and $25 \mathrm{psi}$ with density $0.0732 \mathrm{~g} / \mathrm{ml}$. The sidewalls of the aluminum cell are $0.175 \mathrm{~mm}$ thick with entrance window of $0.18 \mathrm{~mm}$ thick and exit window of $0.132 \mathrm{~mm}$ thick. The two dummy target foils shown are made of aluminum having lengths $4 \mathrm{~cm}$ and $15 \mathrm{~cm}$ are used to subtract background events. The dummy targets are mounted on separate frames with foil located at z positions corresponding to the cryotarget exit and entrance window. The optics target which consists of nine carbon foils cut from 
the same sheet of $99.5 \%$ pure carbon is used for event trajectory reconstruction calibration. The ladder also contains other solids targets such as $\mathrm{Be} 0,{ }^{12} \mathrm{C}$ and a carbon hole target. The carbon hole target is used for beam position calibration whereas the solid carbon target is used for acceptance studies.

\subsection{High Resolution Spectrometer}

The scattered electrons were detected in the high-resolution spectrometers (HRS); one which is positioned along the right side of the beam line called right HRS (RHRS) and the other positioned along the left side of the beamline called left HRS (LHRS). Both spectrometers were used to detect electrons in single arm mode during the run of the GMp experiment. Each HRS contains three quadrupoles and a dipole in QQDQ configuration. The first quadrupole Q1, focuses vertically, whereas, the second Q2, and third Q3, focus in the horizontal plane. The dipole has a vertical bend of $45^{\circ}$, which provides the momentum resolution at $10^{-4}$ level. The dipole separates the charged particle with respect to their

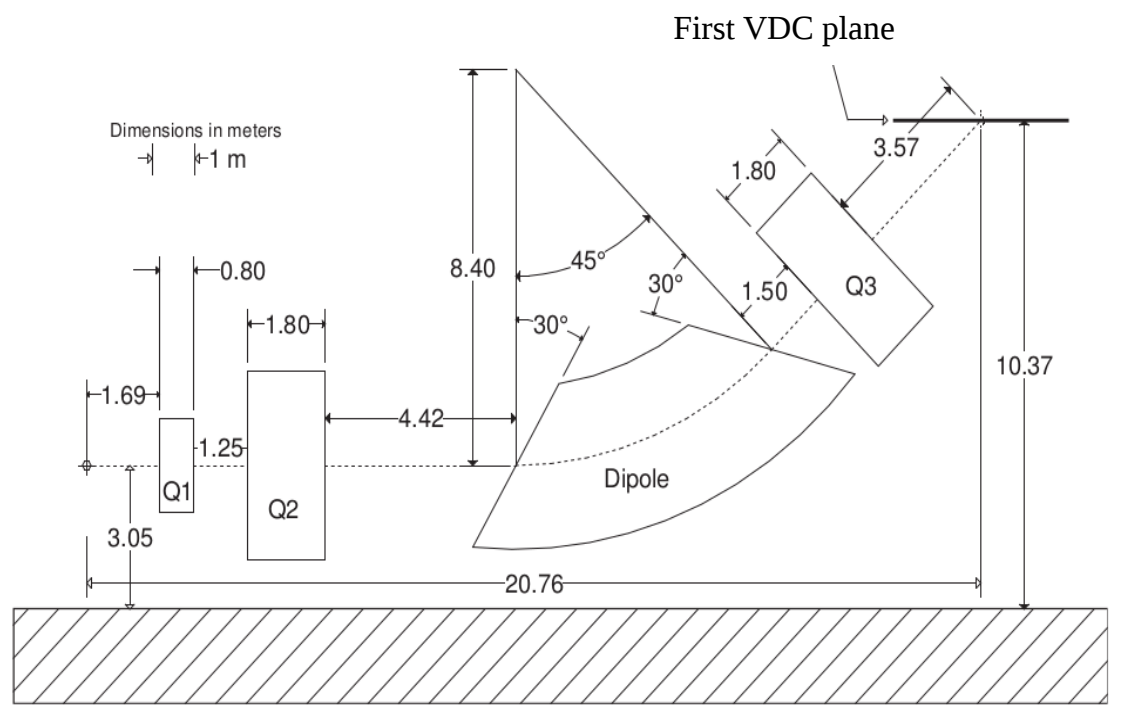

Figure 2.11. Magnet configuration of the Hall A HRS spectrometers showing the sizes and locations of the dipole and the quadrupoles [10].

momenta. The relation between spectrometer central momentum and dipole field strength 
is given by

$$
p_{0}=\sum_{i=1}^{3} B_{0} \gamma_{i}
$$

Where, $\gamma$ is the spectrometer constant and $B_{0}$ is the dipole field. These constants were determined for both HRSs over their full range [42]. The main characteristics of the Hall A HRSs are summarized in the table 2.1 [10].

Table 2.1

The characteristics of the Hall A HRSs.

\begin{tabular}{|l|l|}
\hline configuration & QQDQ \\
\hline Momentum range & $0.3-4.0 \mathrm{GeV} / \mathrm{c}$ \\
\hline Momentum acceptance & $\pm 4.5 \%$ \\
\hline Momentum resolution & $1 \times 10^{-4}$ \\
\hline Optical length & $23.4 \mathrm{~m}$ \\
\hline Bend angle & $45^{\circ}$ \\
\hline Horizontal angular acceptance & $\pm 28 \mathrm{mrad}$ \\
\hline Vertical angular acceptance & $\pm 60 \mathrm{mrad}$ \\
\hline Solid angle & $6 \mathrm{msr}$ \\
\hline Horizontal angular resolution & $0.6 \mathrm{mrad}$ \\
\hline Vertical angular resolution & $2.0 \mathrm{mrad}$ \\
\hline
\end{tabular}

\subsection{HRS Detector Package}

The detector packages for the two spectrometers were almost identical during the GMp experiment. The configuration of each package is shown in Fig. 2.12. Each package has two vertical drift chambers (VDCs) for particle tracking, one straw chamber to resolve the uncertainty in tracking efficiency, and two planes of scintillators for triggering the detector readout. Additionally, each package contained a $\mathrm{CO}_{2}$ gas threshold Cherenkov detector and two arrays of lead glass blocks in a pre-shower and shower configuration for particle identification. 


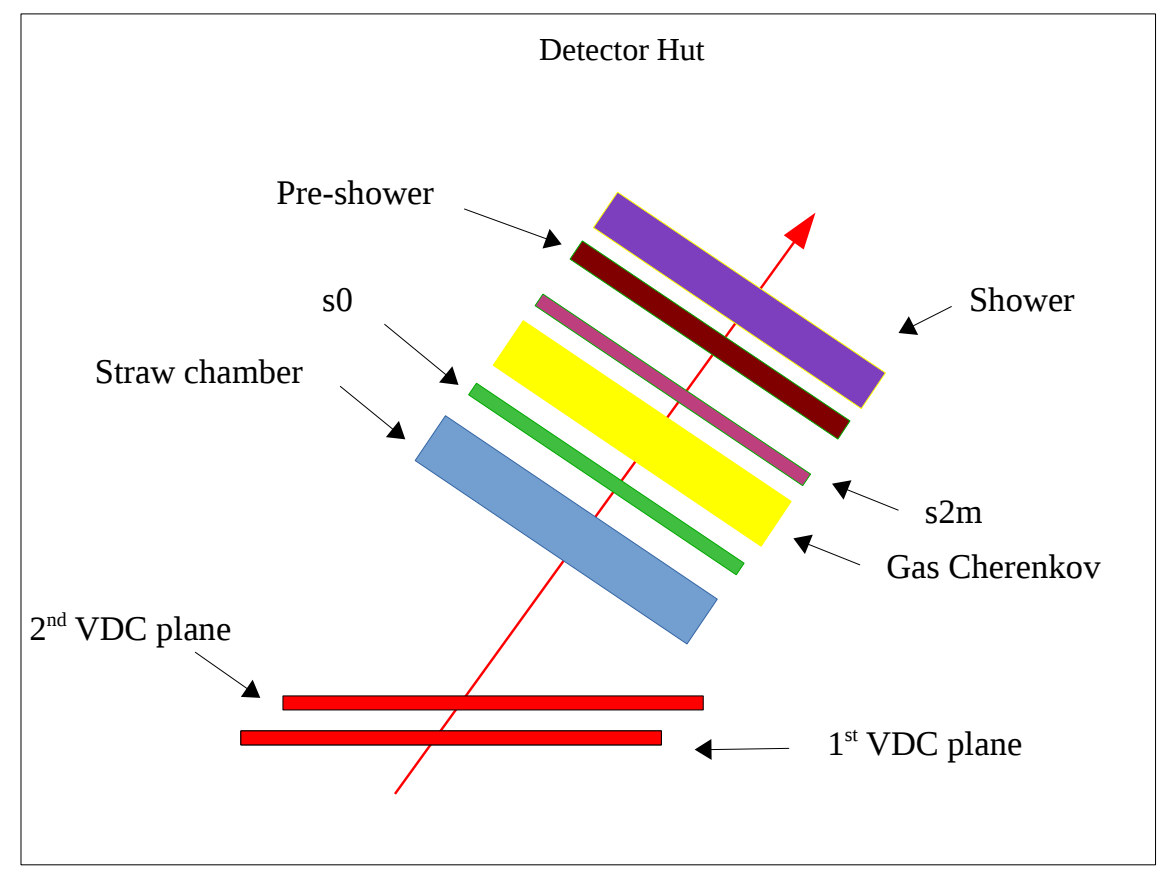

Figure 2.12. Schematic of HRS Detector stack.

\subsection{Vertical Drift Chamber}

The vertical drift chambers are used to determine the trajectory of the incident particle at the detector package, which is then transported back to the target using the known optical properties of the spectrometer to get the momentum and scattering angle at the vertex. The configuration is shown in Fig. 2.13. The two VDCs are $33.5 \mathrm{~cm}$ apart from each other. Each VDC has two wire planes and in each plane, wires are oriented at $45^{\circ}$ or $-45^{\circ}$ with respect to the scattered particle trajectory. The wires in each successive plane are orthogonal to each other. The chambers are filled with a gas mixture of argon (62\%) and ethane (38\%) by volume. Each plane has 368 parallel gold coated tungsten wire sandwiched between gold coated Myler planes powered at the voltage of $-4 \mathrm{kV}$. The tungsten wires are grounded.

When a charged particle crosses the VDC planes it ionizes the gas and the released electrons drift along the electric field lines. Once the electron is near a wire, where the field becomes strongest an avalanche of electrons is created inducing a signal. The signal then get amplified, 

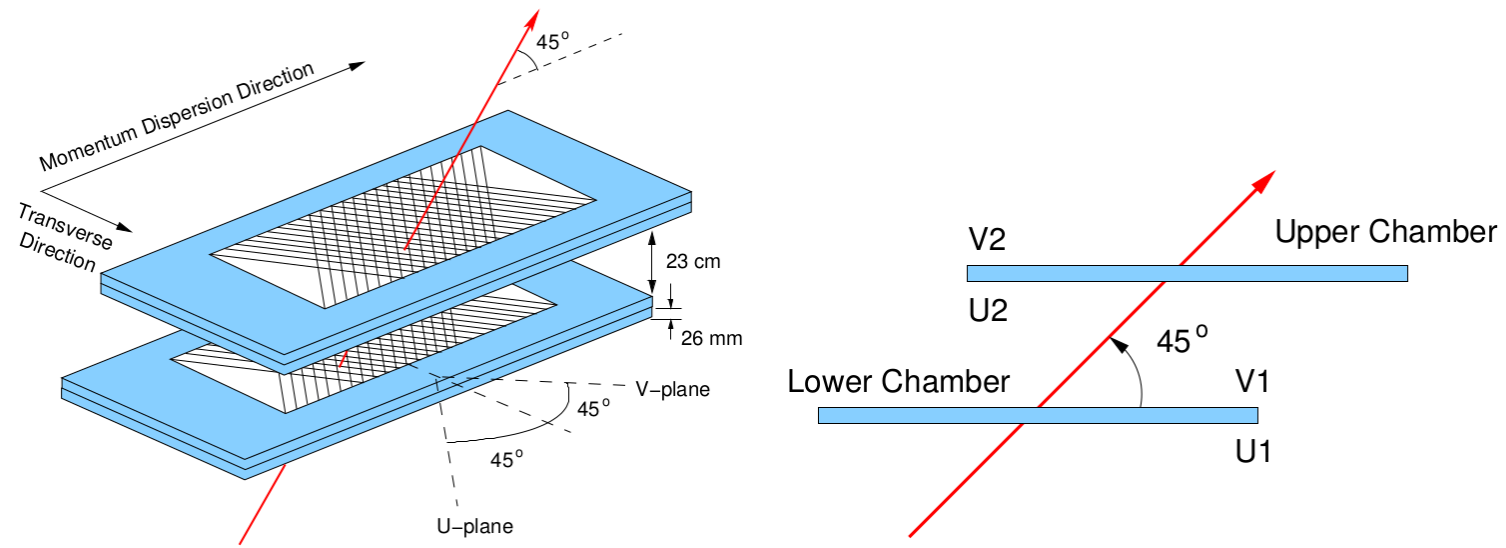

Figure 2.13. Schematic diagram of the VDC wire planes showing a side view of the VDC pair (right figure) and the orientation of the wires relative to the central ray (left figure). Figure is taken from [11].

discriminated and then sent to a multihit time to digital converter (TDC), which is started by the sense wire and stopped by the event trigger. On average, five wires have read-out signals when a particle passes through each wire plane. The trajectory is extracted by using timing information from the TDC to determine drift distances for each wire in a cluster. The cross-over point that the track pass through the wire plane is then determined by a linear fit of drift distances verses wire position.

\subsection{Coordinate Systems}

There are five coordinate systems in experimental Hall A. A detailed description of the coordinate systems used in the reconstruction of trajectories is given below. 


\subsubsection{Hall Coordinates}

The origin of the Hall coordinate system is the center of the Hall, which is on the beamline. The $\hat{z}$ axis is along the beam direction; $\hat{y}$ points vertically upwards and $\hat{x}$ is towards the left side of the beam direction in the horizontal plane as shown in Fig. 2.14.

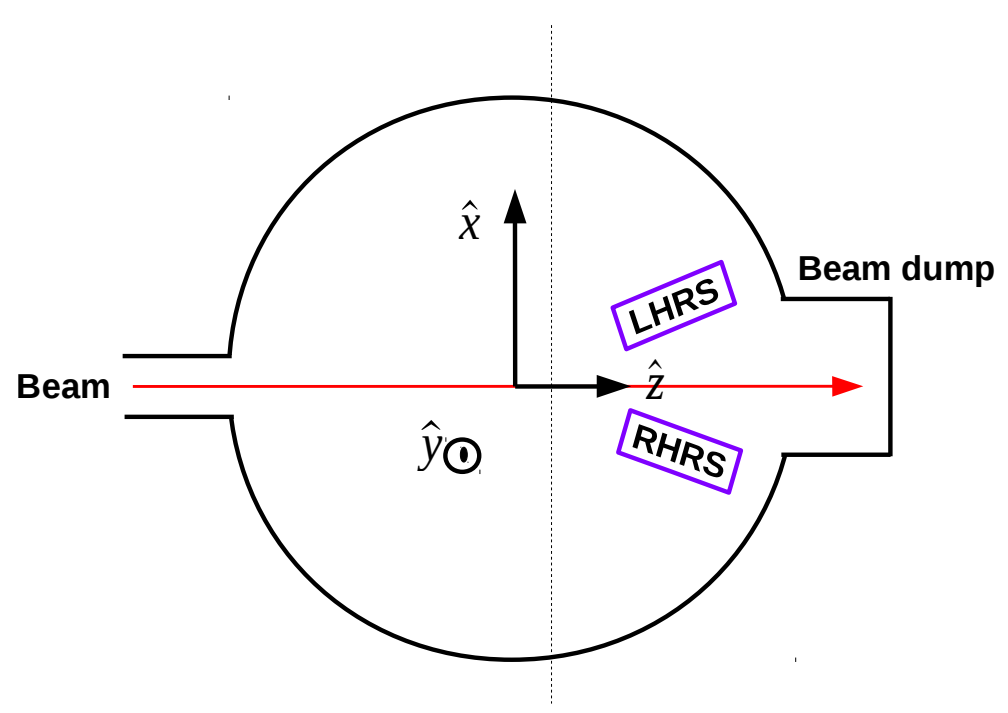

Figure 2.14. Hall Coordinate system (top view).

\subsubsection{Target Coordinates}

The target coordinate system (TCS) moves with the spectrometer. The $\hat{z_{t g}}$ axis is the line perpendicular to the sieve slit of each spectrometer, pointing towards the central hole in the sieve plane. The $\hat{y_{t g}}$ is pointing to the left, whereas $\hat{x_{t g}}$ pointed vertically down. Ideally, the $\hat{z_{t g}}$ axis should pass through the hall center if the spectrometer points directly to the hall center and the sieve slit is perfectly centered. However, the origin of the TCS deviates from the hall center and the deviation can be measured by surveying the miss-pointing. There are two basic target variables: the in-plane angle $\left(y_{t a r}^{\prime}\right)$ and the out-of-plane angle $\left(x_{t a r}^{\prime}\right)$, which 
are used to calculate the full scattering angle in the TCS, via

$$
\theta=\arccos \left(\frac{\cos \theta_{0}-y_{t a r}^{\prime} \sin \theta_{0}}{\sqrt{1+x_{t a r}^{\prime}+y_{t a r}^{\prime}}}\right)
$$

where $\theta_{0}$ is the angle between $\hat{z_{t g}}$ of TCS and the $\hat{z}$ of the hall coordinates and is called central spectrometer angle.

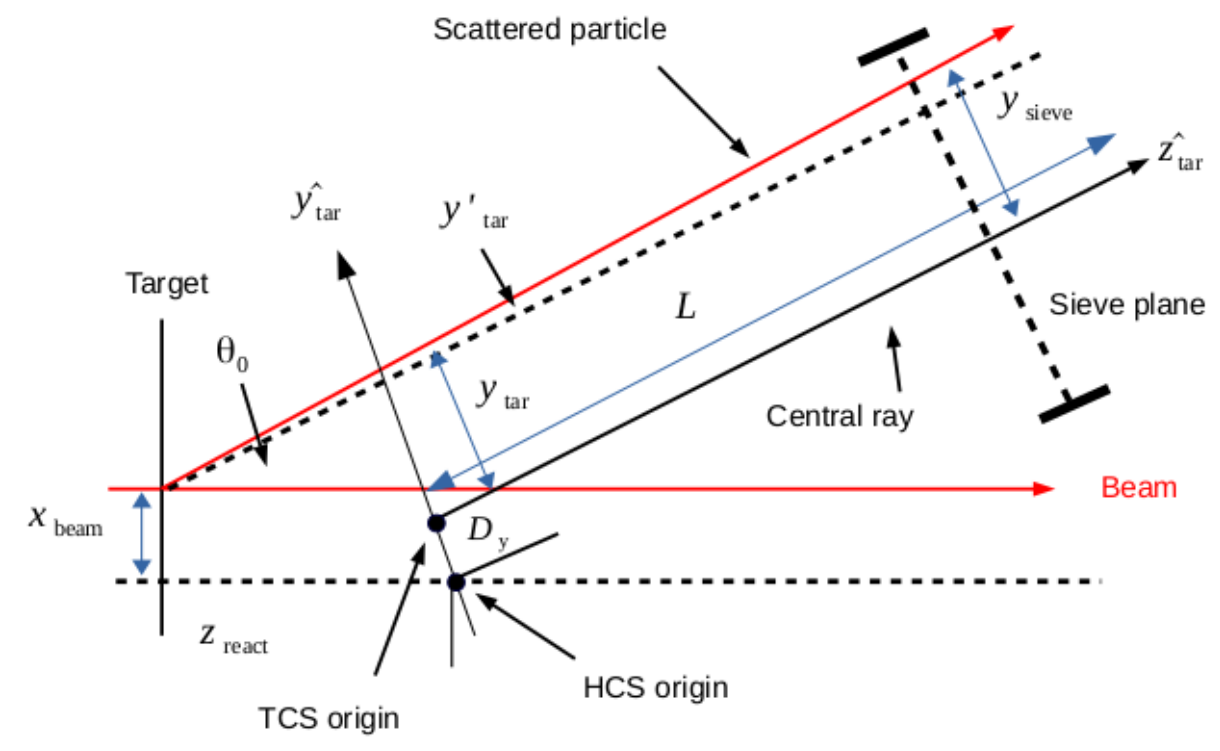

Figure 2.15. Top view of the Target Coordinate system. L is the distance from the hall center to the sieve plane, while D is miss-pointing.

\subsubsection{Detector Coordinate System}

The directions and positions of the trajectories at the focal plane are defined in the detector coordinate system (DCS). The origin of the DCS is given by the intersection of wire 184 of the VDC1 U1 plane and the perpendicular projection of wire 184 in the VDC1 V1 plane onto the VDC1 U1 plane. $z_{\hat{d e t}}$ is perpendicular to the U1 plane and points up, $y_{\text {det }}$ is parallel to the short symmetry axis of the lower VDC and $x_{\text {det }}$ is parallel to the long 
symmetry axis of the lower VDC pointing downstream. The angles $\tan x_{\text {det }}^{\prime}$ and $\tan y_{\text {det }}^{\prime}$ are

Top view

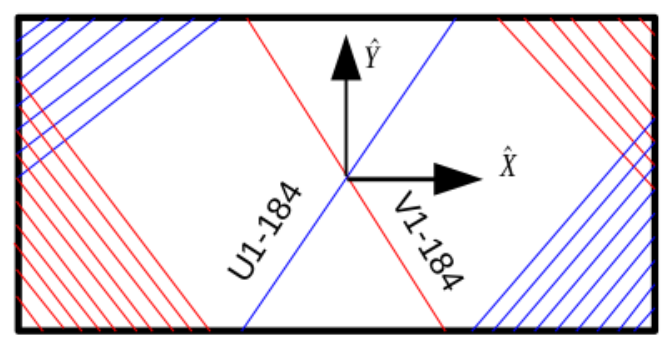

Side view

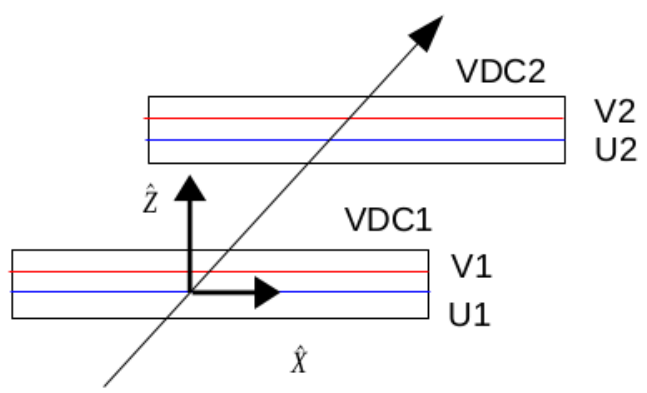

Figure 2.16. Detector coordinate system (top and side views). The intersection point of the wire 184 of the U1 plane and the perpendicular projection of wire 184 of the V1 plane onto the $\mathrm{U} 1$ plane is shown in the top view.

defined as

$$
\begin{aligned}
& \tan x_{d e t}^{\prime}=\frac{d x_{d e t}}{d z_{d e t}} \\
& \tan y_{d e t}^{\prime}=\frac{d y_{d e t}}{d z_{d e t}}
\end{aligned}
$$

\subsubsection{Transport Coordinate System}

The transport coordinate system at the focal plane is generated by rotating the DCS around the y axis by $45^{\circ}$ clockwise so that the new $\hat{z}$ direction is along the central ray. The TCS of an event can be calculated from the DCS coordinates by:

$$
\begin{aligned}
x_{t r a} & =x_{d e t} \cos \left(\rho_{0}\right)\left(1+x_{t r a}^{\prime} \tan \left(\rho_{0}\right)\right. \\
x_{\text {tra }}^{\prime} & =\frac{x_{d e t}^{\prime}+\tan \left(\rho_{0}\right)}{1-x_{d e t}^{\prime} \tan \left(\rho_{0}\right)}
\end{aligned}
$$




$$
\begin{aligned}
& y_{\text {tra }}=y_{\text {det }}+\sin \left(\rho_{0}\right) y_{\text {tra }}^{\prime} x_{\text {det }} \\
& y_{\text {tra }}^{\prime}=\frac{y_{\text {det }}^{\prime}}{\cos \left(\rho_{0}\right)\left(1-x_{d e t}^{\prime} \tan \left(\rho_{0}\right)\right)}
\end{aligned}
$$

where $\rho_{0}=-45^{\circ}$.

\subsubsection{Focal Plane Coordinate System}

The focal plane coordinate system (FCS) is obtained by rotating DCS clockwise around $y_{\text {det }}$ axis through an angle $\rho$, where $\rho$ is the angle between the local central ray and $z_{\text {det }}$ axis of the DCS $\left(x_{t a r}^{\prime}=y_{t a r}^{\prime}=0\right)$ for the corresponding relative particle momentum). Because of the focusing of the HRS magnet system, particles with the same scattering momentum will be focused at the focal plane, which means the relative momentum to the central momentum of the spectrometer is approximately a function of $x_{t r a}$. The main feature of the FCS is that the dispersive angle will be small and thus making the expressions for optics reconstruction converge faster. The FCS also includes corrections for the offsets due to misalignment in the VDC. So, in the FCS, the coordinates and angles of the trajectory can be written as:

$$
\begin{aligned}
& x_{f p}=x_{t r a} \\
& x_{f p}^{\prime}=\frac{x_{d e t}^{\prime}+\tan (\rho)}{1-x_{d e t}^{\prime} \tan (\rho)} \\
& y_{f p}=y_{t r a}-\sum_{i} C_{i 000}^{y} x_{f p}^{i} \\
& y_{f p}^{\prime}=\frac{y_{d e t}^{\prime}-\sum_{i} C_{i 000}^{p} x_{f p}^{i}}{\cos (\rho)\left(1-x_{d e t}^{\prime} \tan (\rho)\right)}
\end{aligned}
$$

Where, $\tan (\rho)=\sum_{i} C_{i 000}^{t} x_{f p}^{i}$. The coefficients $C_{i 000}^{t}, C_{i 000}^{y}$ and $C_{i 000}^{p}$ also include corrections for the systematic offsets due to misalignment of VDC packages. 


\subsection{Straw Chamber}

The straw chamber consist (SC) of a set of tubes with a thin wire running along the central axis of the tube as shown in Fig. 2.17. It is filled with a gas mixture of $62 \%$ argon and $38 \%$ of ethane by volume. The GMp experiment used two straw chambers, one in each

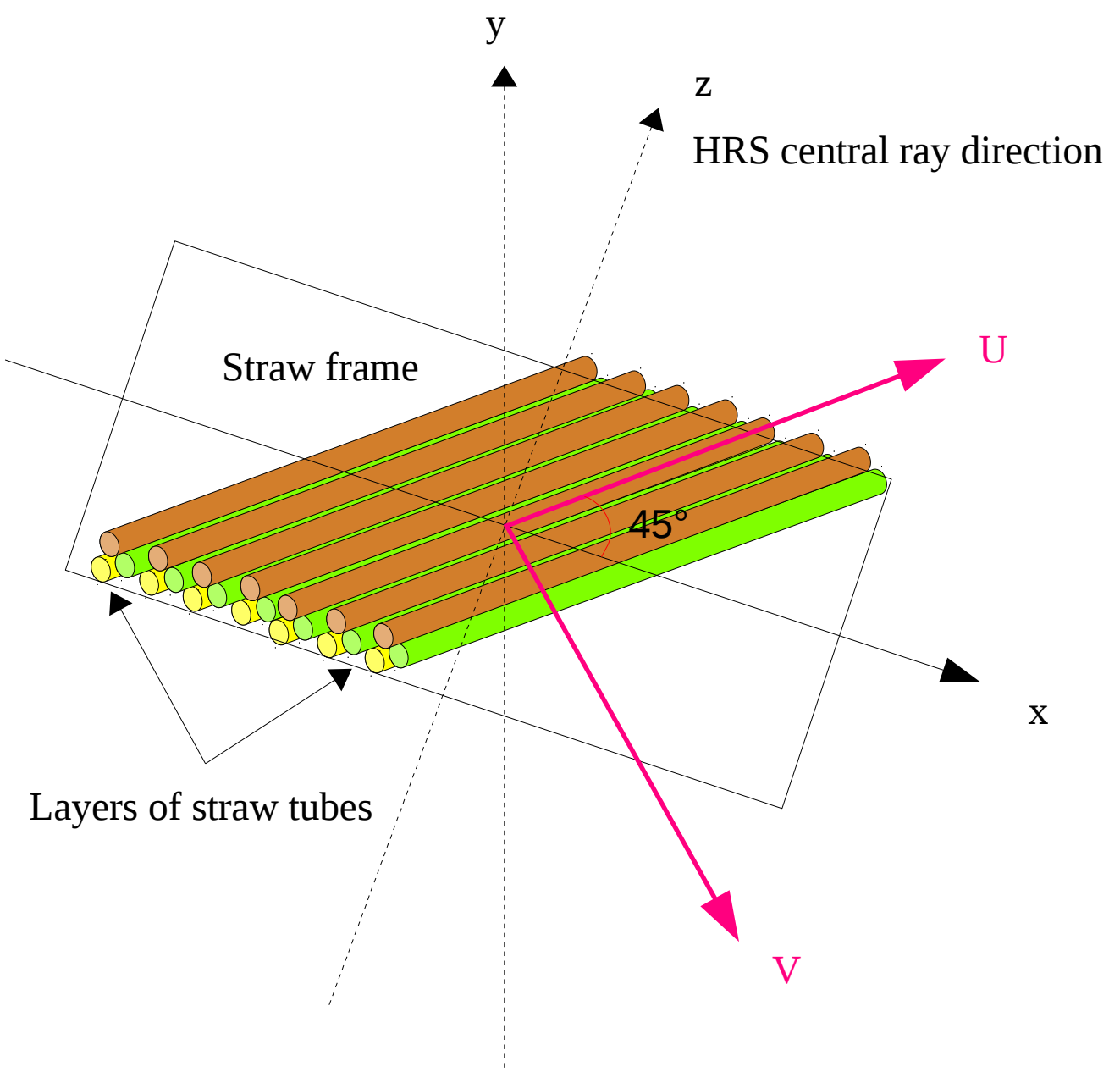

Figure 2.17. Schematic diagram of three U planes of straw tubes. Here $\mathrm{x}$ is along dispersive direction of HRS and $\mathrm{z}$ is along the HRS central ray. The V planes are located at the bottom of the straw frame not shown on the graph.

HRS arm as a spare tracking plane to find the particle track parameters in the presence of multiple clusters in the VDCs. The SC was installed between the VDCs and cherenkov detector. Each straw consists of six planes, $3 \mathrm{U}$ and $3 \mathrm{~V}$ planes. The $\mathrm{U}$ and $\mathrm{V}$ direction are at $+45^{\circ}$ and $-45^{\circ}$ relative to the dispersive direction in the $x-y$ plane. There are 176 
tubes in each U plane and 160 tubes in each V plane with configuration UUUVVV. During the operation, the central anode wire is kept at a voltage of $+1800 \mathrm{~V}$ whereas the tubes are grounded. When the charged particle passes through SC tube it ionizes the gas mixture and the produced ions and electrons travel to the anode wire and the tube cell respectively to produce a signal at the wire.

\subsection{Scintillator Planes}

The GMp experiment utilized the s0 and s2m scintillator planes in each spectrometer in Hall A. The s0 scintillator is located before the gas Cherenkov and the s2m is located after the gas Cherenkov detector on each HRS. When a charged particle passes through a paddle of the scintillator, it produces light that travels towards both ends of the paddle. The two scintillator planes are about 1.6 meters apart. $\mathrm{S} 0$ is composed of one thin plastic sintillation paddle while s2m has 16 paddles. Each paddle is viewed by two photomultipliers (PMTs), one at each end. For s0, a coincidence between top and bottom PMTs was made whereas, for s2m plane, the logical OR of the PMTs were formed individually for the right 16 PMTs and the left 16 PMTs and then logical AND was formed between them. The main trigger for the spectrometer is referred to as T1 which and was formed by logical AND of the s0 and s2m logic pulses. This trigger is then sent to the trigger supervisor, which determines whether the data acquisition system (DAQ) should record the particular event. By determining the time of flight of the particle between the scintillator planes from the TDC signals, and by knowing the distance between the two scintillator planes, the velocity of the particle $v$ or $\beta=\frac{v}{c}$ can be determined. The $\beta$ of the particle is used for particle identification since different charged particles of different masses and known momentum can be separated. 


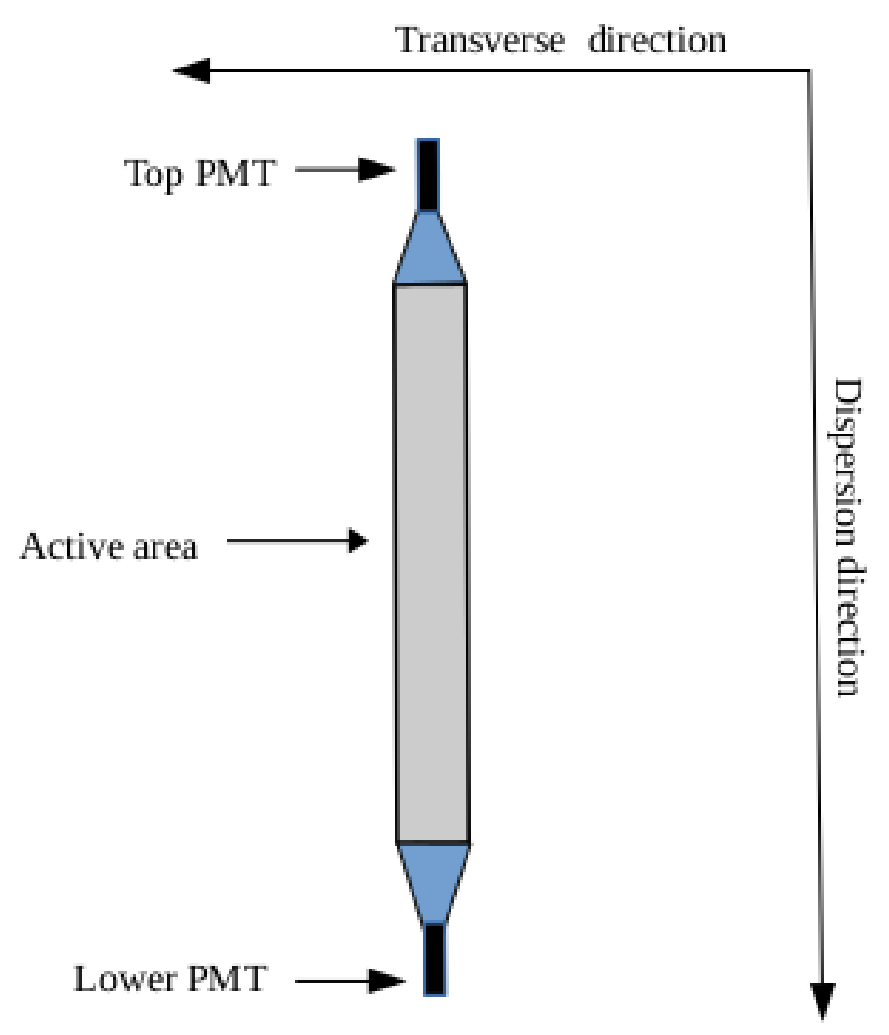

Figure 2.18. Layout of the s0 scintillator plane.

\subsection{Gas Cherenkov}

The HRS gas Cherenkov detector provides particle identification by distinguishing between electrons and pions utilizing Cherenkov radiation. As shown in Fig. 2.20, it is a rectangular tank filled with $\mathrm{CO}_{2}$ at atmospheric pressure and is installed between the scintillators $\mathrm{s} 0$ and s2m on each arm. This detector measures the light emitted when a charged particle travels through the material of refractive index $n$ with velocity $v$ greater than the speed of light in the material. This radiation is known as Cherenkov radiation. The Cherenkov radiation occurs when

$$
v \geq c_{m}
$$




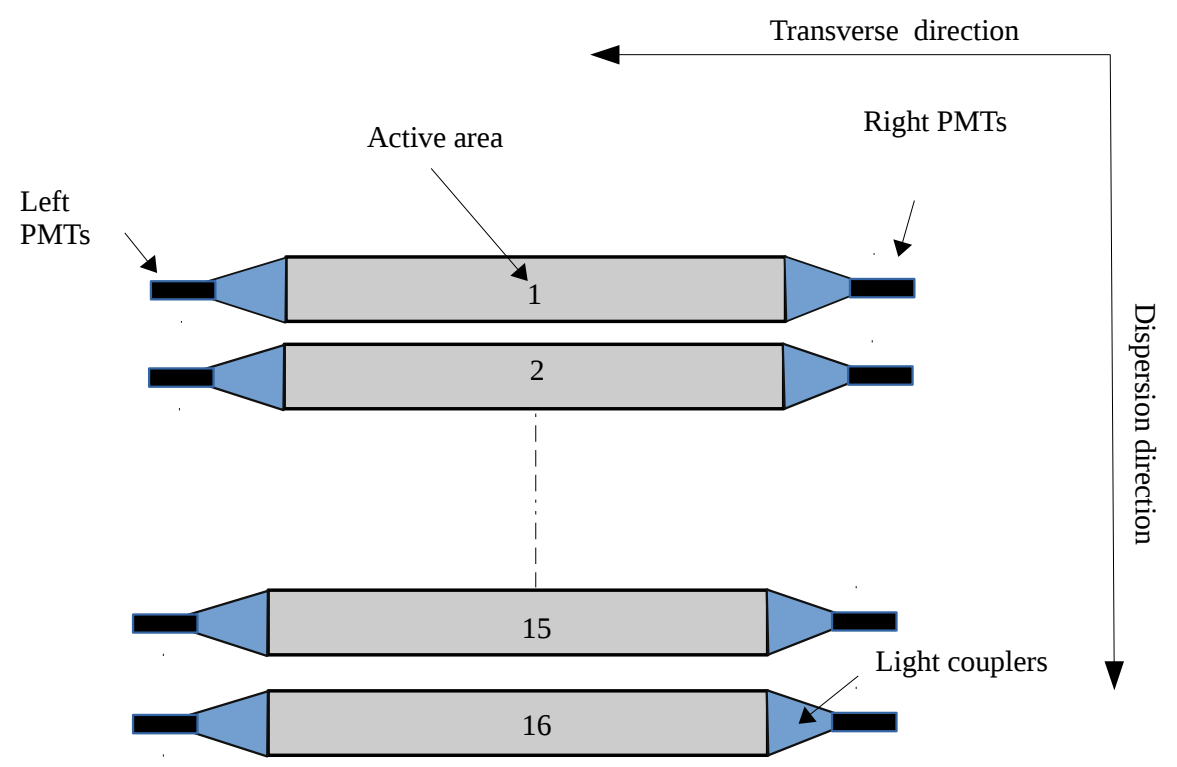

Figure 2.19. Layout of the s2m scintillator plane.

Where, $c_{m}=c / n$ is the speed of light in material. The Hall A Cherenkov detector consists of 10 mirrors that reflect the Cherenkov light, placed just before the detector exit window. Each mirror has a spherical surface with a radius of curvature of $90 \mathrm{~cm}$ and reflect light to PMTs placed at the side of the detector box. If $v \leq c_{m}$ then the polarization of the medium is symmetric about the path of the particle. In this case, the net dipole moment is zero, and no radiation occurs. However, when $v \geq c_{m}$, the asymmetric polarization of the medium leads to a non zero dipole moment, as a result of which Cherenkov radiation is emitted. The threshold momentum for a particle can be obtained from:

$$
P_{t h}=\frac{m c_{m}}{\sqrt{n^{2}-1}}
$$

For $\mathrm{CO}_{2}$ gas, the refractive index $n=1.00041$, and the threshold momentum for electrons is $17 \mathrm{MeV} / \mathrm{c}$, whereas, for pions, this is $4.8 \mathrm{GeV} / \mathrm{c}$, which is greater than the HRS momentum range for this experiment. So, only electrons give a signal in Cherenkov detector. In spite of the fact that pions can't emit Cherenkov radiation, they may influence the ADC 

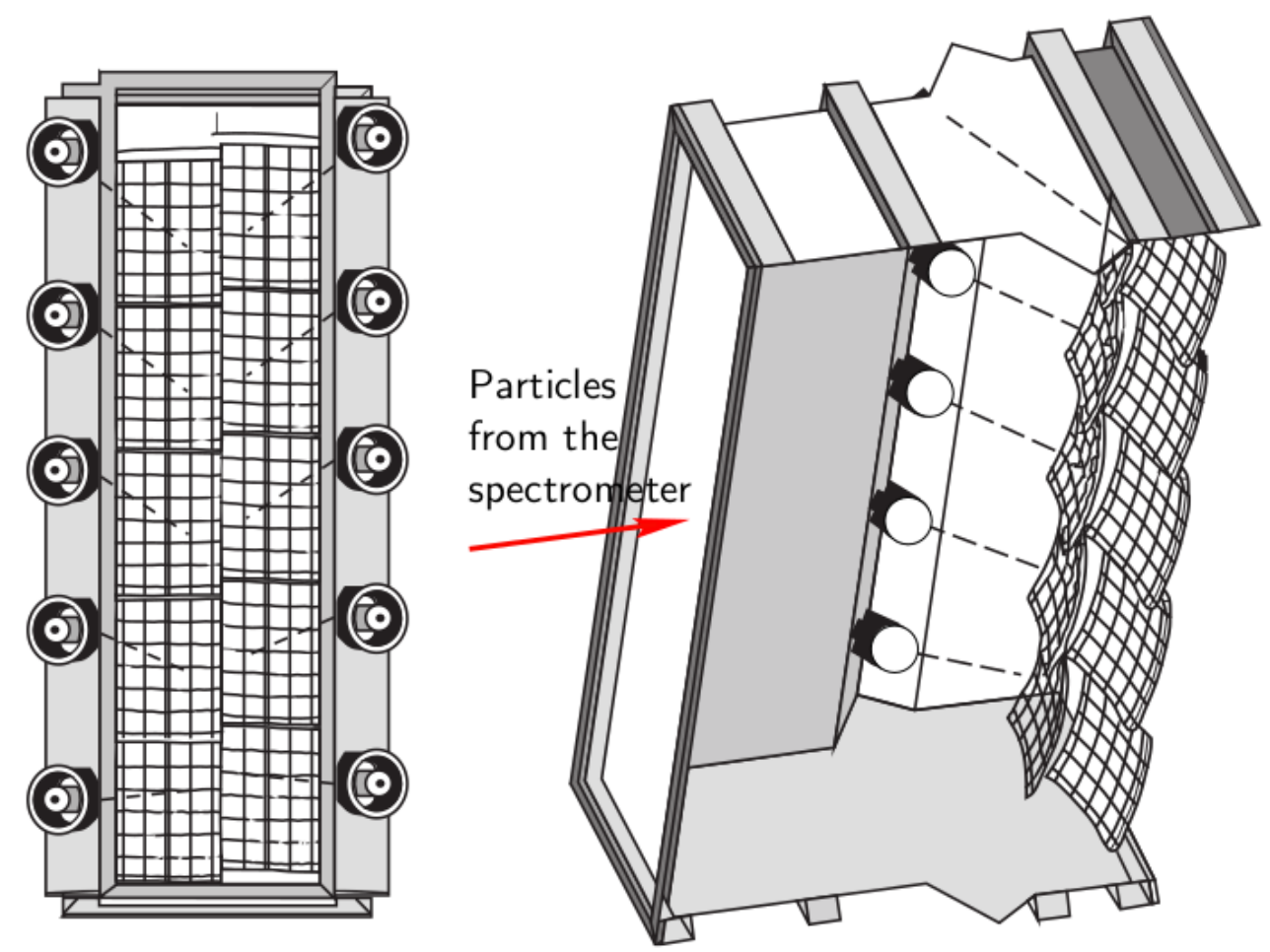

Figure 2.20. Layout of the $\mathrm{CO}_{2}$ Gas Cherenkov detector. Figure is taken from [12].

spectrum. This happens because pions ionize the atoms of the gaseous medium, producing electrons that can trigger the detector. Such electrons are called $\delta$-ray electrons. Fig. 2.21 shows distribution of number of photoelectrons in the gas Cherenkov. The events around 1 photoelectron peak represents the thermal electrons from the photocathode. The red line indicates the boundary between the electron and pion region. The pion events are removed with the addition of a lead glass calorimeter.

\subsection{Lead Glass Calorimeter}

In addition to the Cherenkov detector, the lead glass calorimeter was also used for rejecting events triggered by pions. The schematic diagram of calorimeter is shown in Fig. 2.22. This is an electromagnetic calorimeter used to detect the energy deposited by charged particle 


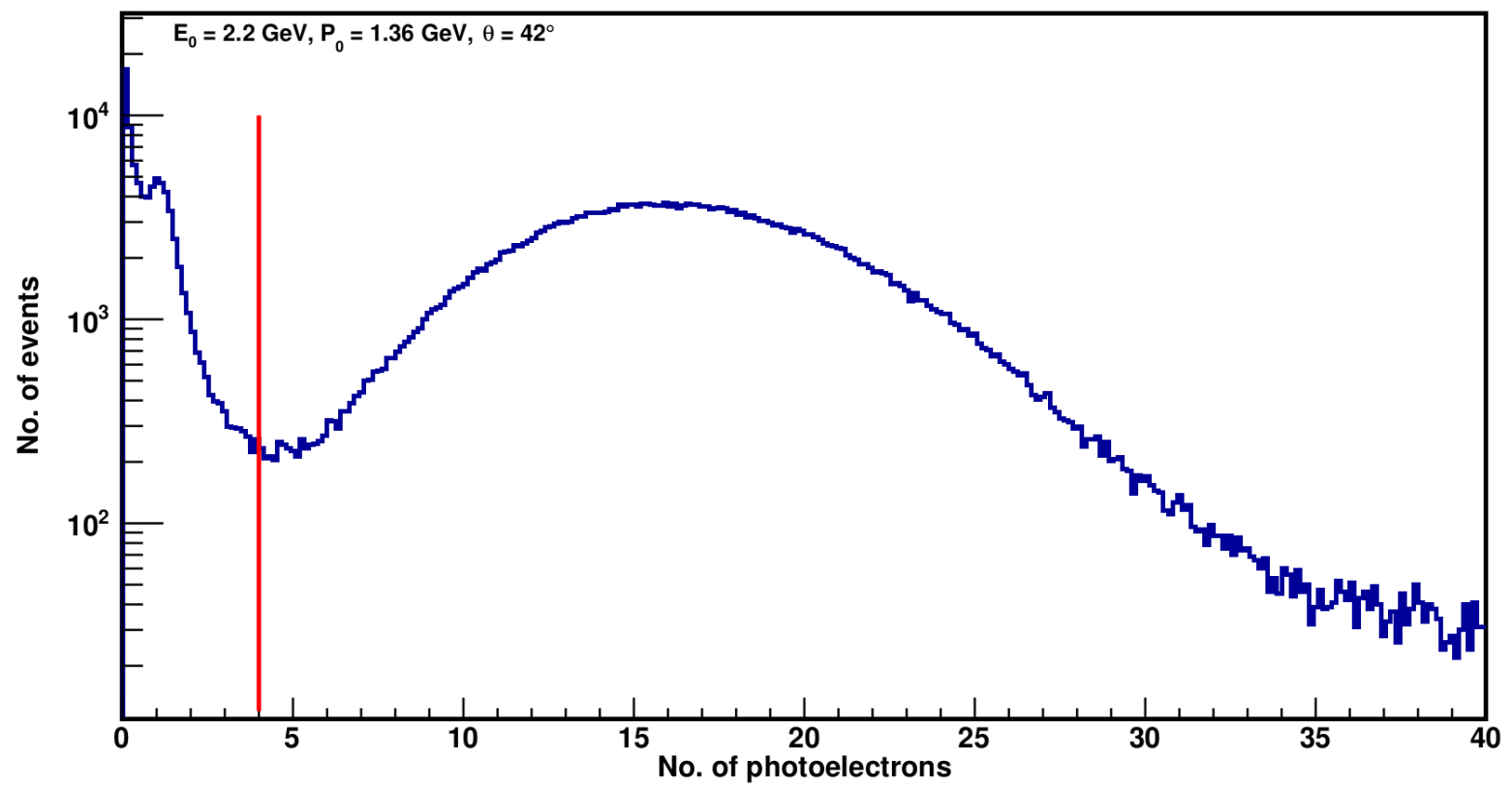

Figure 2.21. Number of photoelectrons collected in the gas cherenkov detector. Red line represents the cut to select good electrons for Fall 2016 LHRS runs.

through Bremsstrahlung and pair production. Bremsstrahlung is electromagnetic radiation produced due to the acceleration of charged particles, for instance an electron in the field of an atomic nucleus. These photons then convert to electron-positron pairs, which in turn radiate photons by interacting with the atoms of the medium, thereby producing further electron-positron pairs. This shower process continues until all the electron energy is deposited in the calorimeter. The radiation produced is then detected by the PMTs at the end of the block. Electrons and positrons deposit all of their energy in the calorimeter, whereas pions usually deposit a small amount of energy due to the ionization and direct Cherenkov light.

The calorimeters in both HRS consist of two segmented layers as shown in Fig. 2.22. The particles enter through the bottom layer. On the LHRS, they are called pion rejector 1 and 2 whereas, on the RHRS the first and second layer are called pre-shower and shower respectively. The blocks in both layers in LHRS and the first layer in RHRS are oriented 

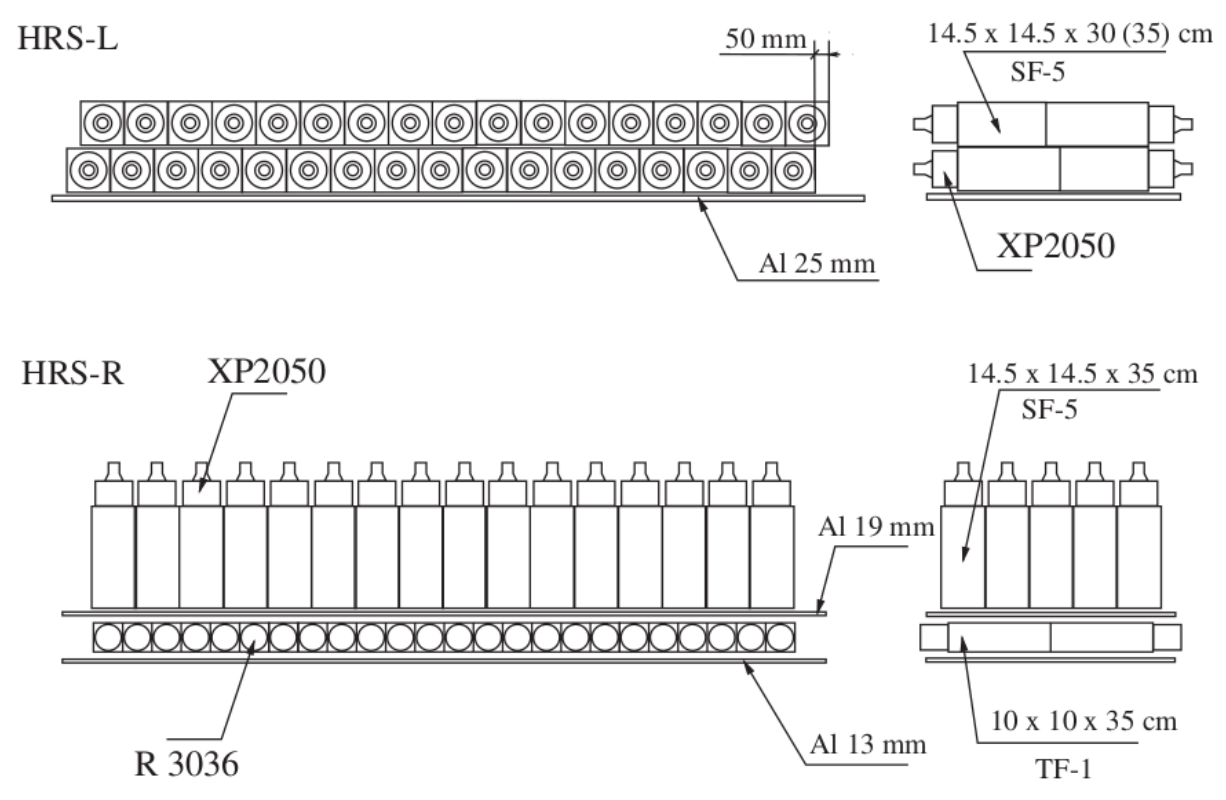

Figure 2.22. HAll A HRS calorimeter detector geometry. Particle enters through the bottom of figure.

perpendicular to the particle tracks whereas, the blocks are parallel to the tracks in the second layer of RHRS. In the left HRS each layer has 17 short blocks and 17 long blocks of lead glass forming a $2 \times 17$ array of dimensions $14.5 \mathrm{~cm} \times 14.5 \mathrm{~cm} \times 30 \mathrm{~cm}$ as shown in Fig. 2.22. In the RHRS the pre-shower has $2 \times 24=48$ blocks, $10 \mathrm{~cm} \times 10 \mathrm{~cm} \times 35 \mathrm{~cm}$ and the shower has $5 \times 16$ blocks of the lead glass, $14.5 \mathrm{~cm} \times 14.5 \mathrm{~cm} \times 35 \mathrm{~cm}$. There are three peaks in the spectrum of the output of the lead glass calorimeter. As in Fig. 2.23 the larger peak corresponds to the electron and the smaller peak at about 0.4 corresponds to the pion.

\subsection{Data Acquisition and Trigger System}

A block diagram of the Hall A DAQ (Data Acquisition) system is shown in Fig. 2.24. The DAQ software is based on CODA (CEBAF Online Data Acquisition) [43] package developed by the Jlab CODA group. Hardware components include, the Trigger Supervisor (TS) module, Analog to digital converters (ADCs), Time to digital converters (TDCs) and scaler 


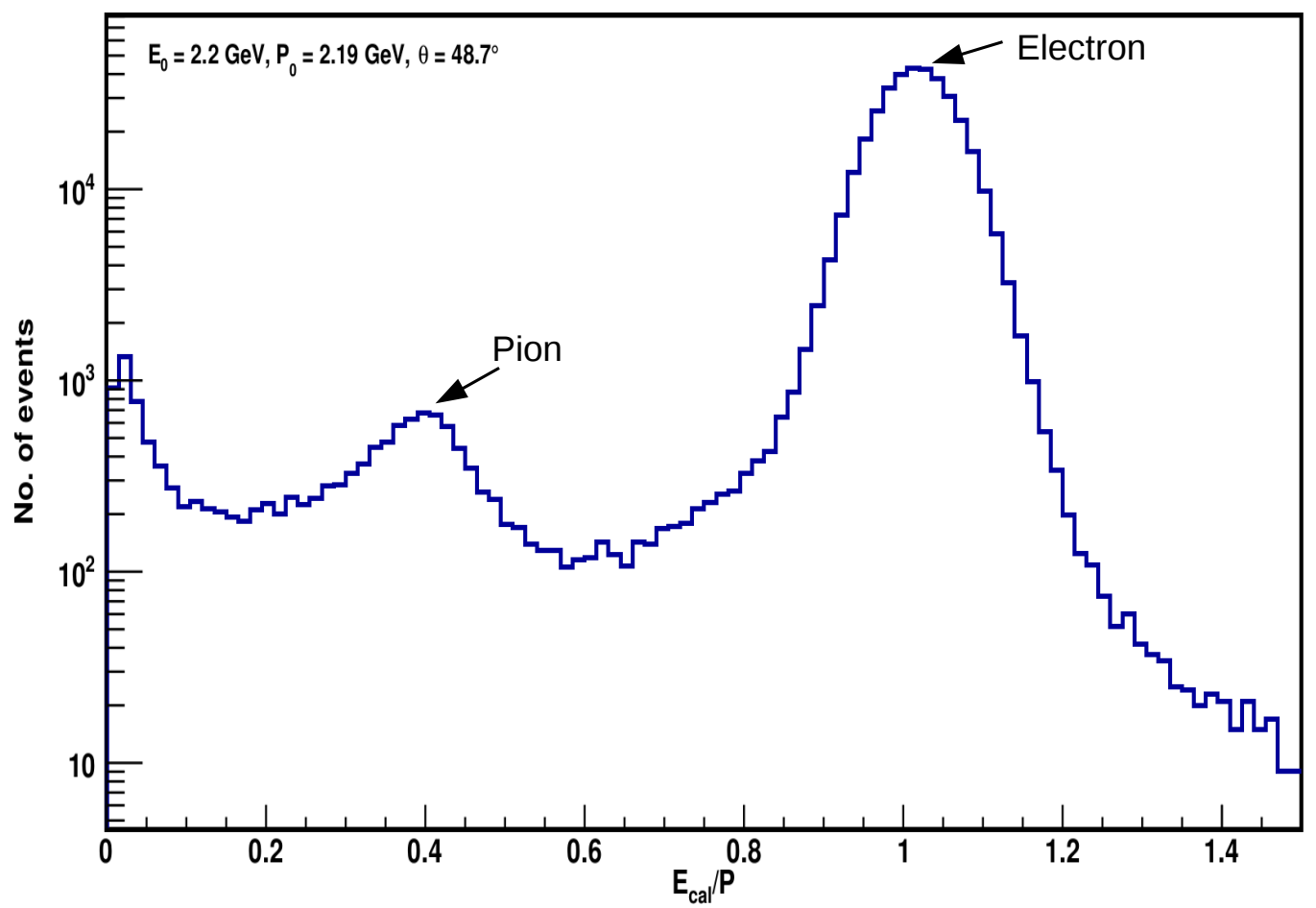

Figure 2.23. The total energy deposited in the calorimeter per unit momentum. The electron peak is at 1 and pion peak is at about 0.4 .

modules. The TDC, ADC and scaler modules are either Fastbus or VME-type. After entering hits from the detectors, the crates are read out by read out controllers (ROC's), which are CODA routines running under the VxWorks operating system . The information from the ROCs are then passed over to the event builder (EB) which is a routine that waits for the connection requested from ROCs, collects their fragments, orders and merges these into a single data structure in CODA format. The events are then passed to the ER (Event recorder), Which is a CODA routine that writes an event to disk and are finally transferred to the Mass Storage System. Triggers are the logic signals that assist the DAQ to start 


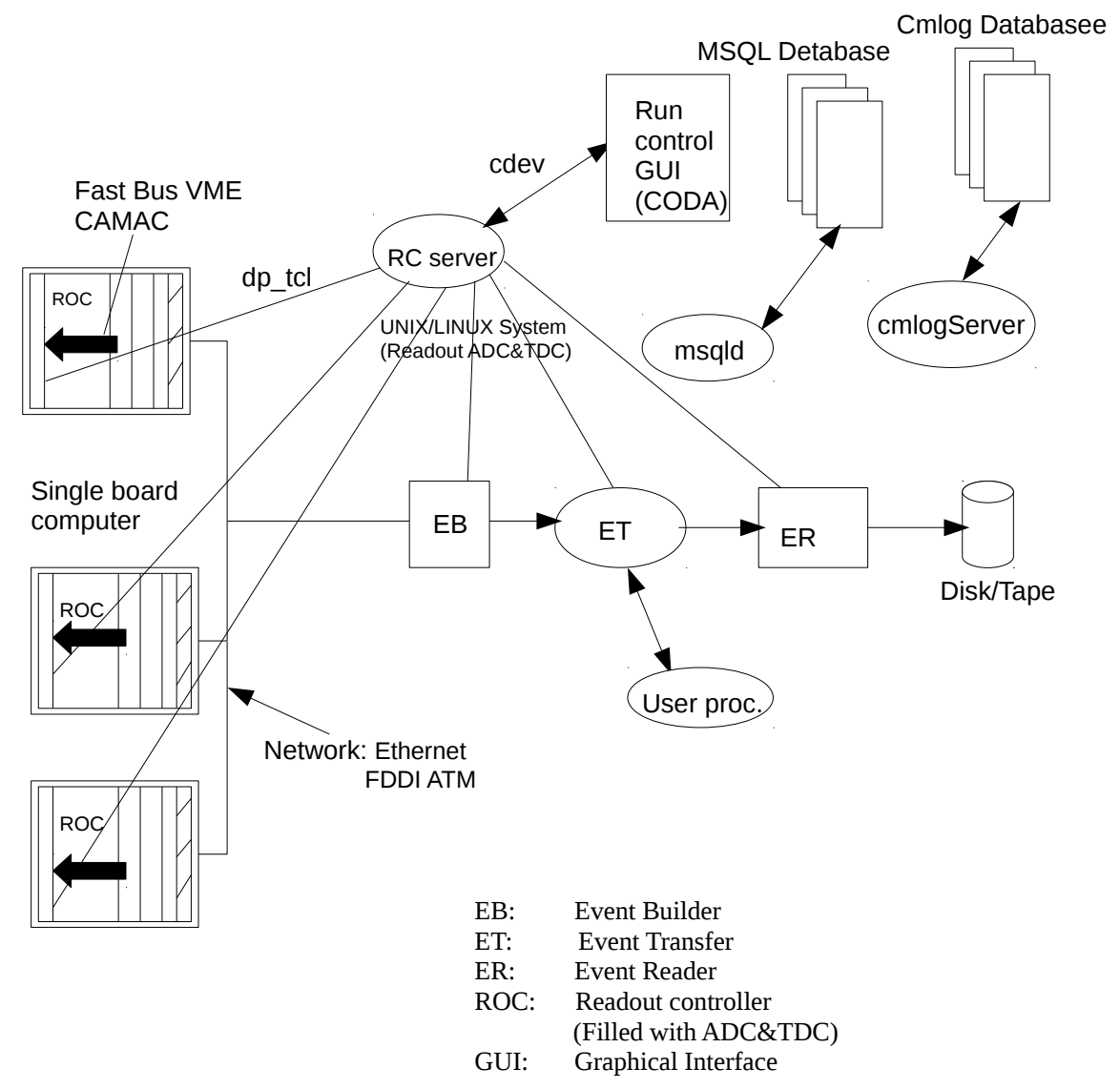

Note: This whole process takes one event at time.

Figure 2.24. Schematics of Hall A DAQ system.

read out of the detectors information. The trigger design for the halla DAQ is shown in Fig. 2.25. Signals from the four detectors: S0 scintillator, S2m scintillator, gas Cherenkov, and calorimeter, are sent to the front-end electronics. Logical pulses from each of these detectors is then sent to a trigger module based on VME programmable logic. This allows various combination of detectors in the trigger. Here the input to the Majority Logic Unit (MLU) [44] also includes Electronic Dead Time Monitor (EDTM) and clock. The outputs of MLU can be adjusted to either form a coincidence between different inputs or simply copy inputs. NIM outputs are then fed to the Trigger Supervisor (TS) via NIM_ECL. The TS is located in the electronic hut on the second floor. When a trigger is accepted by the TS, a 


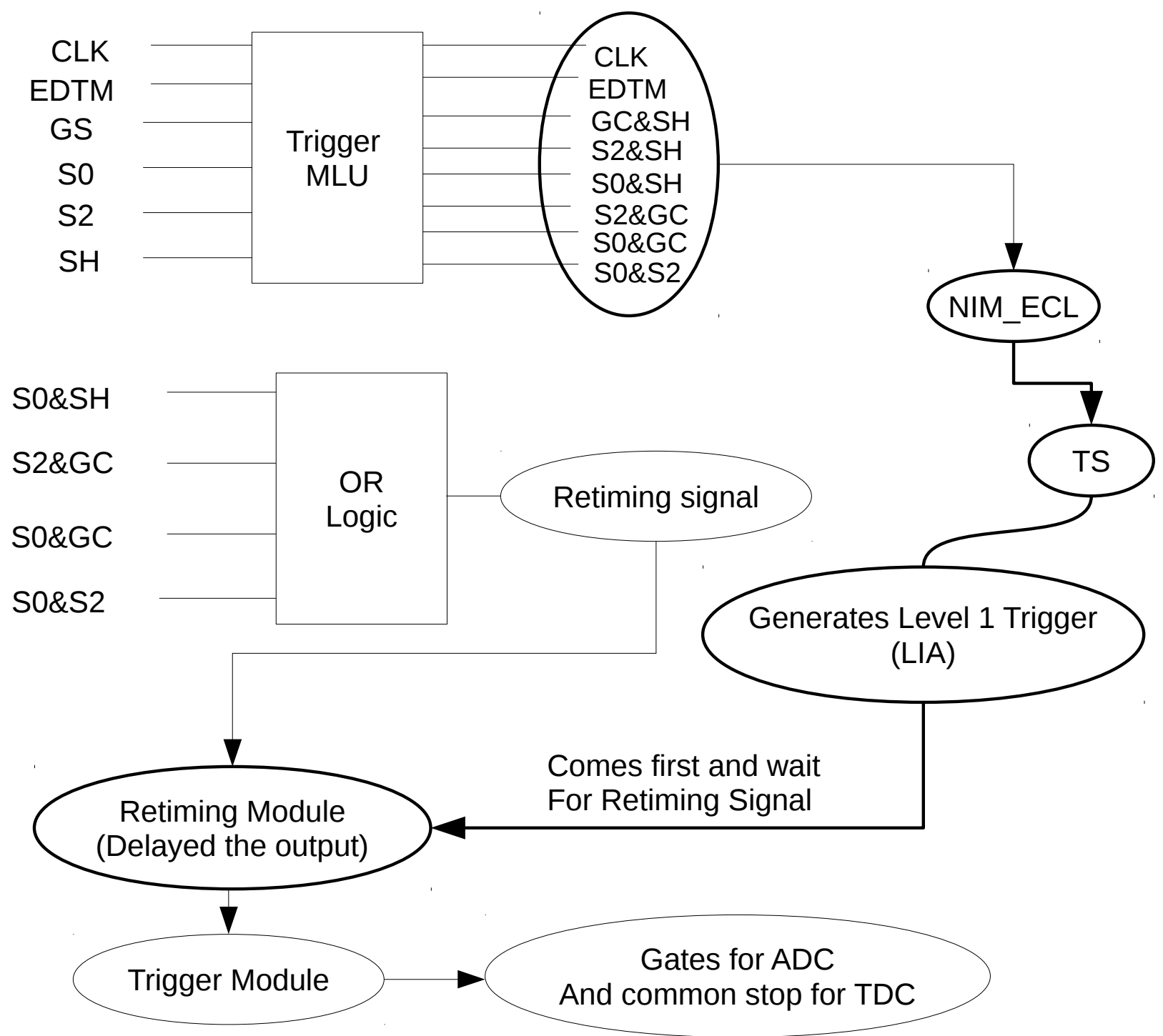

Figure 2.25. Schematics for Hall A trigger system.

level one-accept (L1A) signal is generated. L1A and the retiming signal are sent to retiming module where they form a coincidence. Then the coincidence signal is fed to the trigger module (TM) where an ADC gate, and a TDC start/stop are generated. These are then distributed to the front end of the electronics on the fast bus crate and VME crates where ADC, TDC and scalers start to record data. The main trigger for each spectrometer of the GMp experiment was formed by the logical AND of the s0 and s2m planes, which is called 
T1. T2 (T3) was formed by the coincident signal of the Gas Cherenkov signal and s0 (s2m) to measure the trigger efficiency. 


\section{CHAPTER 3 \\ DATA ANALYSIS}

\subsection{Overview}

In scattering experiments, the cross sections are determined from the number of counts in bins in scattered momentum and angle divided by the number of incoming electrons and the number of possible scattering center per unit area. Therefore, to determine the elastic cross section the counts are integrated and corrected for any inefficiency. In addition, cuts on PID detectors are implemented to ensure that only electrons are counted. The basic procedure for extracting elastic cross sections from experimental data is flow charted in Fig 3.1. For analysing data the Hall $\mathrm{A}$ a $\mathrm{C}++$ software package [45] has been utilized to convert the raw data in the form of ADC Channels, TDC channels, and scaler counts to ROOT files [46] to store the detector information and the reconstructed physics variables. The BPMs were calibrated to give an accurate measurement of the angle of the incoming beam to the target which will be discussed in next section. The well calibrated BCMs were utilized to convert the scaler counts to beam charge. The detailed discussion of the BCM calibration will be discussed in the section 3.3. The dead times associated with the DAQ system were evaluated to recover the events lost during the data acquisition. The calibrated HRS optics matrix taking the tracks reconstructed at the detector were used to reconstruct the scattered electron's momentum and scattering angle at the reaction vertex. This relies on the optics of the spectrometer which will be discussed in the section 3.7. The HRS detectors were calibrated and good electrons were identified by applying the cuts on signals, and the efficiencies of the event selection were determined individually. Then the background subtracted charge normalized yield corrected to the dead time and detector efficiencies were extracted. For the luminosity, it is important to know the density and the length of the target when it has a beam on it. A target boiling study was performed 
to determine the effect of boiling on the density of cryotarget which will be discussed in the section 3.4. In addition, a Monte Carlo simulation package was utilized to determine the acceptance of the spectrometer which used transport matrices generated by COSY to simulate the spectrometer optics. This package generates simulation events with the same kinematic setting and weight by physics cross section provided by fit to the existing data, including relevant physics processes, energy straggling, multiple scattering etc. The Monte Carlo yield ratio method was used to extract cross sections. Beside this, the acceptance correction method was utilized to check the results obtained from the ratio method. 


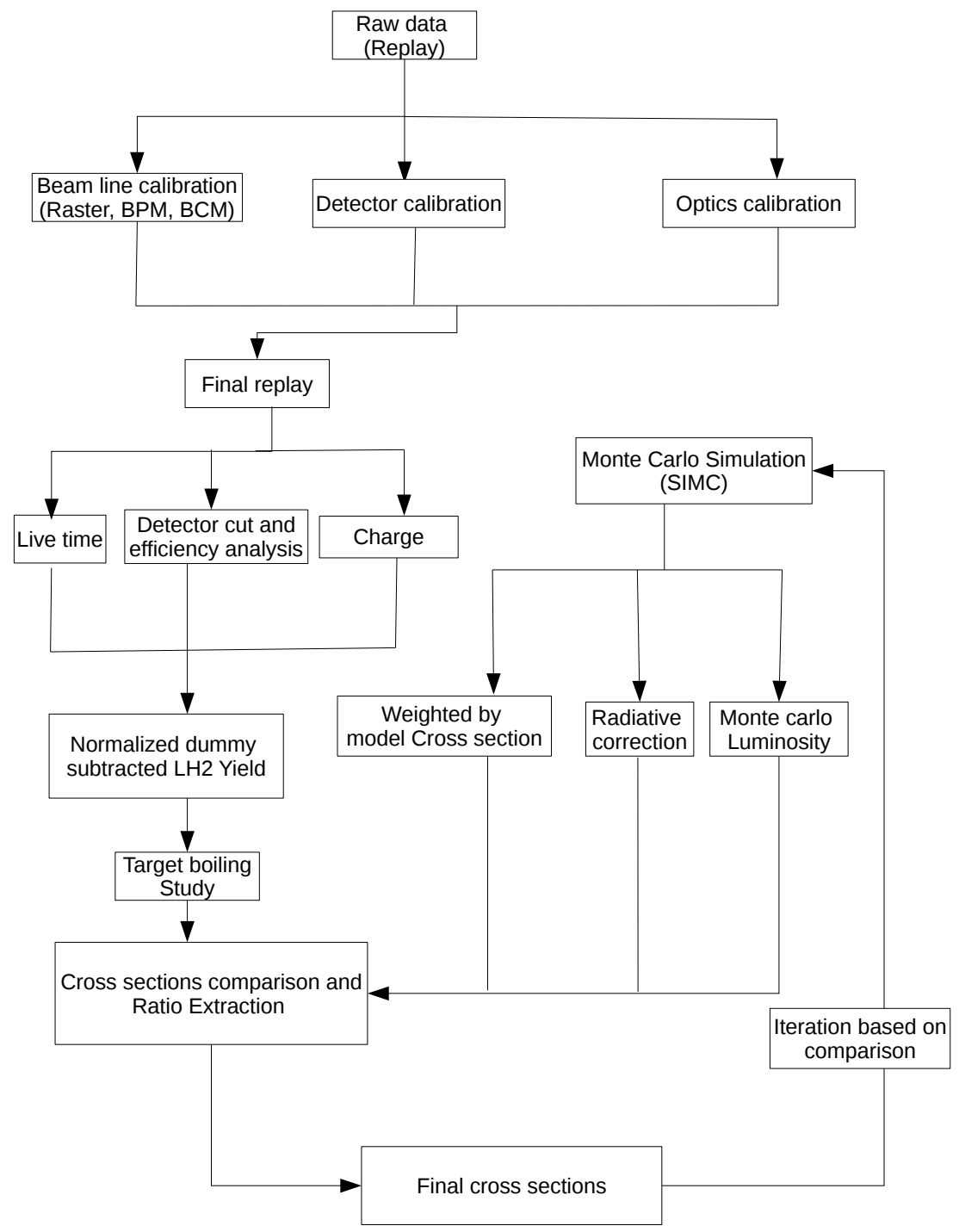

Figure 3.1. Flow chart describing cross section extraction. 


\subsection{BPM Calibration}

As noted in section 2.4.2, the BPMs are utilized to non-invasively monitor the average position of the beam during data taking, after first being calibrated against the Harps. The Harps are also used to measure the intrinsic width and beam position relative to the beamline. This section will discuss the calibration of the BPMs against Harps. The top
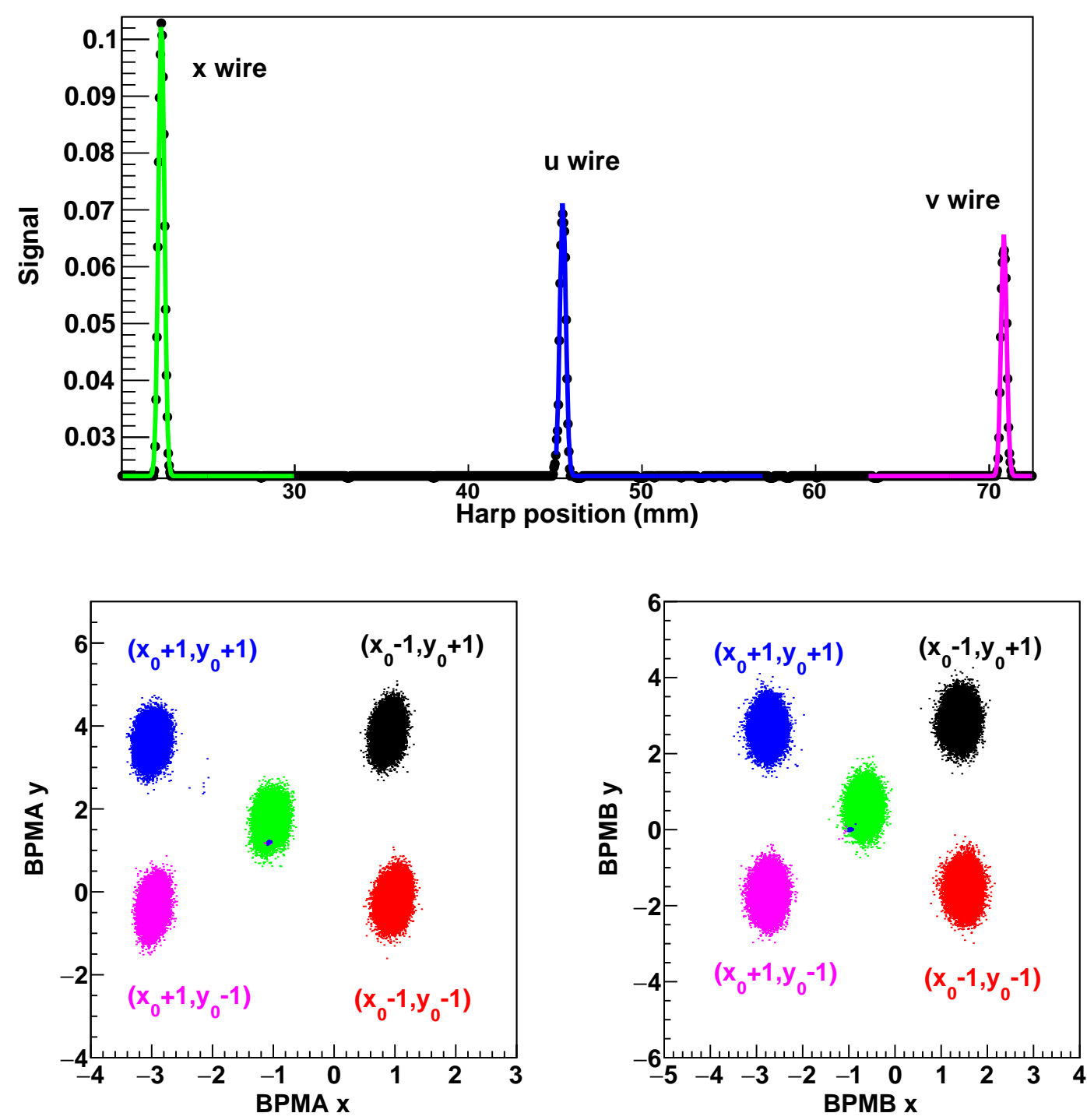

Figure 3.2. Top: Harp wires response versus stepper motor position in $\mathrm{mm}$. The peaks in the output of harp indicate points where the harp wires crossed the beam line. Bottom: Scatter plots of the BPM position in $y$ vs. $x$ for runs in the bulls eye scan. 
panel in Fig 3.2 shows the result for a typical Harp-scan measurement performed during the experiment. Each Harp consists of three thin wires that are moved across the beam path as shown in right panel of Fig. 2.6 in section 2.4.2. The first into the beam is a vertical wire labeled as " $\mathrm{x}$ ". The next two wires are at \pm 45 degrees relative to the vertical and are labeled as "u" and "v". When the frame is moved into the beam using a steeper motor each wire crosses the electron beam and secondary emitted electrons produce a signal that is detected and amplified. This amplified signal together with the position encoder is sent to an ADC which is then read out and stored in a datafile.

For calibration of the BPMs, a "bull's eye scan" is performed using the unrastered beam by moving the beam to five different positions as shown in the bottom panel of Fig 3.2. During the scan, the beam is first centered using the carbon hole target. As soon as the beam becomes stable, a harp scan and CODA run were performed. The beam is then steered to positions around the nominal center in the pattern indicated in the bottom panels of 2.4.2, covering at least the area that the raster will cover. For each position the Harp and coda runs are repeated. The Harp scans were analyzed using the latest survey results to get the corrected beam position relative to the Hall Center. Finally, the calibration constants need to be determined that give the position at the BPM which is closest to the harp using the following equation:

$$
\left[\begin{array}{l}
X \\
Y
\end{array}\right]_{\text {harp }}=\left[\begin{array}{ll}
c_{11} & c_{12} \\
c_{21} & c_{22}
\end{array}\right]\left[\begin{array}{l}
X^{\prime} \\
Y^{\prime}
\end{array}\right]_{B P M}+\left[\begin{array}{l}
x_{o f f} \\
y_{o f f} \cdot
\end{array}\right]
$$

As this is an over determined system (having 5 harp positions), a least squares method was used to calculate the constants, where, $c_{11}, c_{12}, c_{21}$ and $c_{22}$ which are the coefficients related to the gains of two antennas pairs and $x_{o f f}, y_{o f f}$ which are the position offsets of the BPM center relative to the nominal beamline. These values are then inserted into the BPM database to be used in the physics analysis. The upper panel plots in Fig. 3.3 show the fit results of the BPM versus the Harp coordinates for the bull's eye scan after calibrations. 

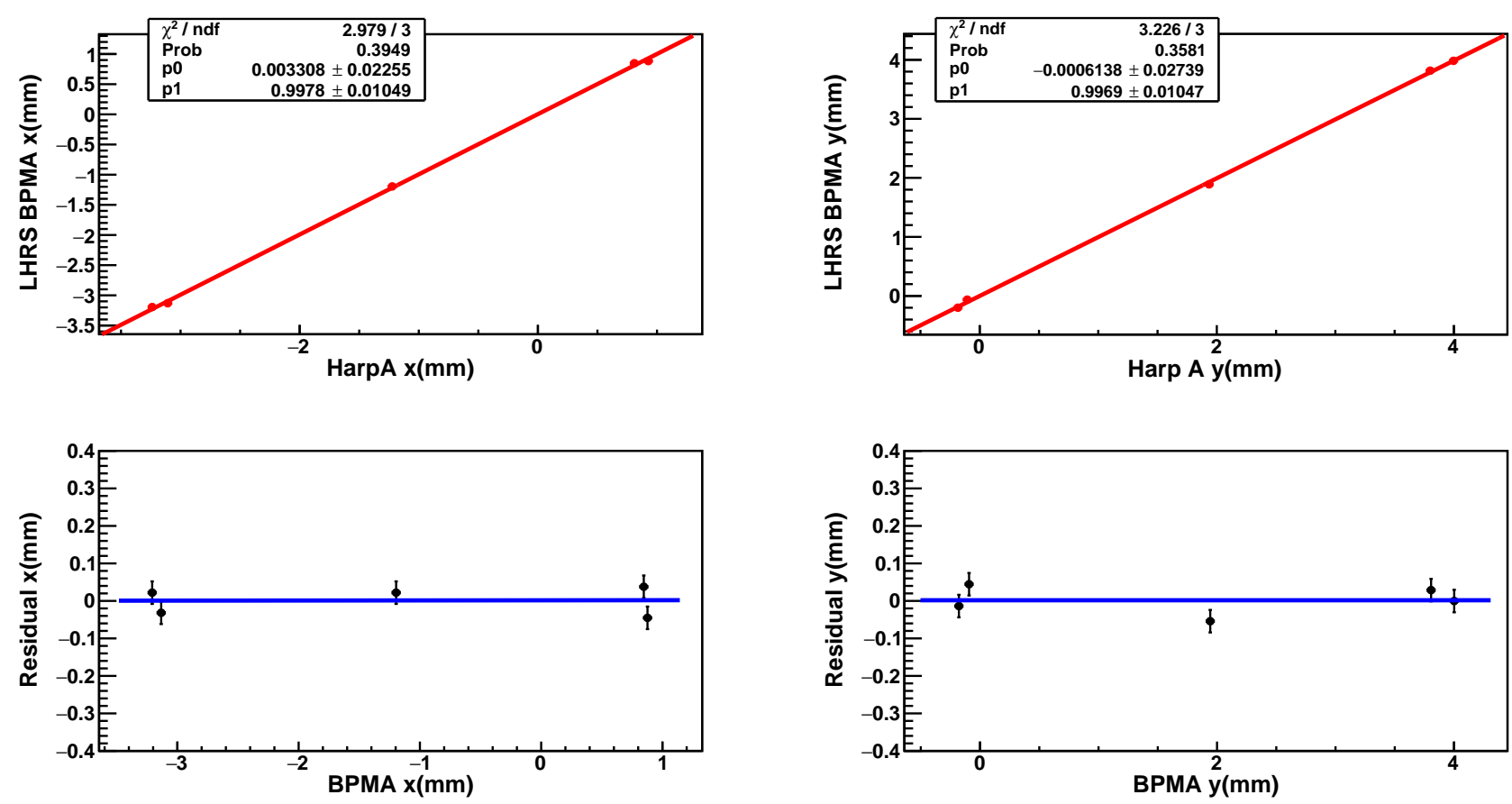

Figure 3.3. Top two plots are the fit results of LHRS BPM A positions vs corresponding Harp positions after calibration, whereas the lower two plots are the residual vs BPM positions

The lower panel plots are the residuals between BPM and Harp coordinates as a function of BPM positions. The uncertainty on the position was estimated to be $0.03 \mu \mathrm{m}$ which yields $\chi^{2}$ values close to unity.

The BPM only provides the average beam position value of the beam. The instantaneous position of the rastered beam is determined from a combination of the BPM readout and the raster current information, event by event. The magnet current of each raster is calibrated to determine the position of the raster with respect to the center of the raster pattern. The raster calibration procedure involves taking the ADC values of raster current and then converting them into position of the beam due to the raster magnet. The detail of raster calibration is presented in Ref. [47]. 


\subsection{Beam Charge Calibration}

The BCM and Unser hardware were discussed earlier in section 2.4.4. The BCMs are used to determine the beam current and charge during data taking. The Unser monitor is used to calibrate the BCMs, since it has a very stable gain, but the offset drifts over the timescales for individual data runs. Here, the results of the beam charge calibration will be presented.

\subsubsection{Unser Wire Calibration}

The Hall A Unser monitor was calibrated with a precisely known current source. The current read-back on the injected current was checked by three other different devices and found to be consistent. At a set current of $30 \mu \mathrm{A}$, the Fluke meter showed $29.94 \mu \mathrm{A}$, the Picoammeter showed $29.965 \mu \mathrm{A}$, and HP meter showed $29.968 \mu \mathrm{A}$. During the Unser calibration, multiple currents were injected through the wire in steps from 0 to $100 \mu \mathrm{A}$ with the time at each current lasting one minute. Additionally, two such ramps were performed as illustrated in the top panel of Fig. 3.4. The bottom panel of Fig. 3.4 shows the injected current versus entry number and the top panel shows the corresponding Unser rate for those entries.

Table 3.1

Unser wire calibration coefficient table from three different run periods.

\begin{tabular}{|l|l|l|}
\hline $\begin{array}{l}\text { Run } \\
\text { number }\end{array}$ & $\begin{array}{l}\text { Gain } \\
(\mu \mathrm{A} / \mathrm{Hz})\end{array}$ & $\begin{array}{l}\text { Error } \\
(\mu \mathrm{A} / \mathrm{Hz})\end{array}$ \\
\hline 12323 & 2753 & 6.1 \\
\hline 21590 & 2754 & 6.1 \\
\hline 22324 & 2753 & 6.1 \\
\hline 10453 & 2759 & 5.5 \\
\hline 22324 & 2753 & 6.1 \\
\hline 23779 & 2504 & 4.5 \\
\hline 23217 & 2506 & 4.7 \\
\hline
\end{tabular}



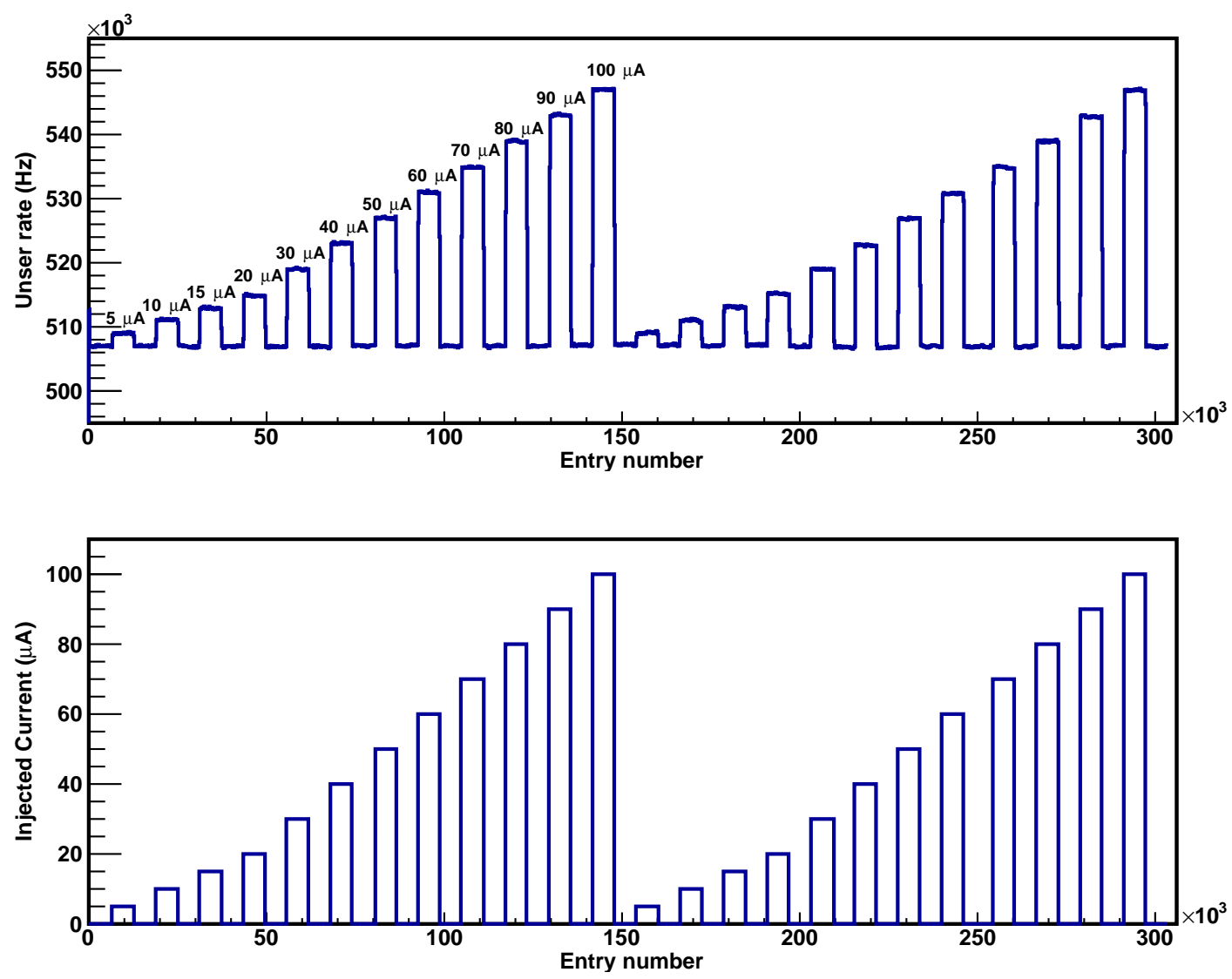

Figure 3.4. The bottom panel shows injected current vs entry number and the top panel shows the corresponding Unser current for those entries during Unser calibration. The time interval for each current-off and current-on period was 1 minute.

Each current-on period is alternated with a current-off period to determine the Unser offset for each period. The average frequency for adjacent current-off periods were subtracted from the average frequency for the current-on to correct for any offset drift. The left panel in Fig. 3.5 shows the Unser frequency fitted against the injected current, with the uncertainty estimated form the current noise as stated by the manufacturer of Unser monitor of $2 / \sqrt{\text { time }}$ $(\mu A / \sqrt{s e c})$.

The right panel in Fig. 3.5 shows the residuals of the Unser current as determined from

$$
I=\left(f_{\text {unser }}-f_{0}\right) G
$$



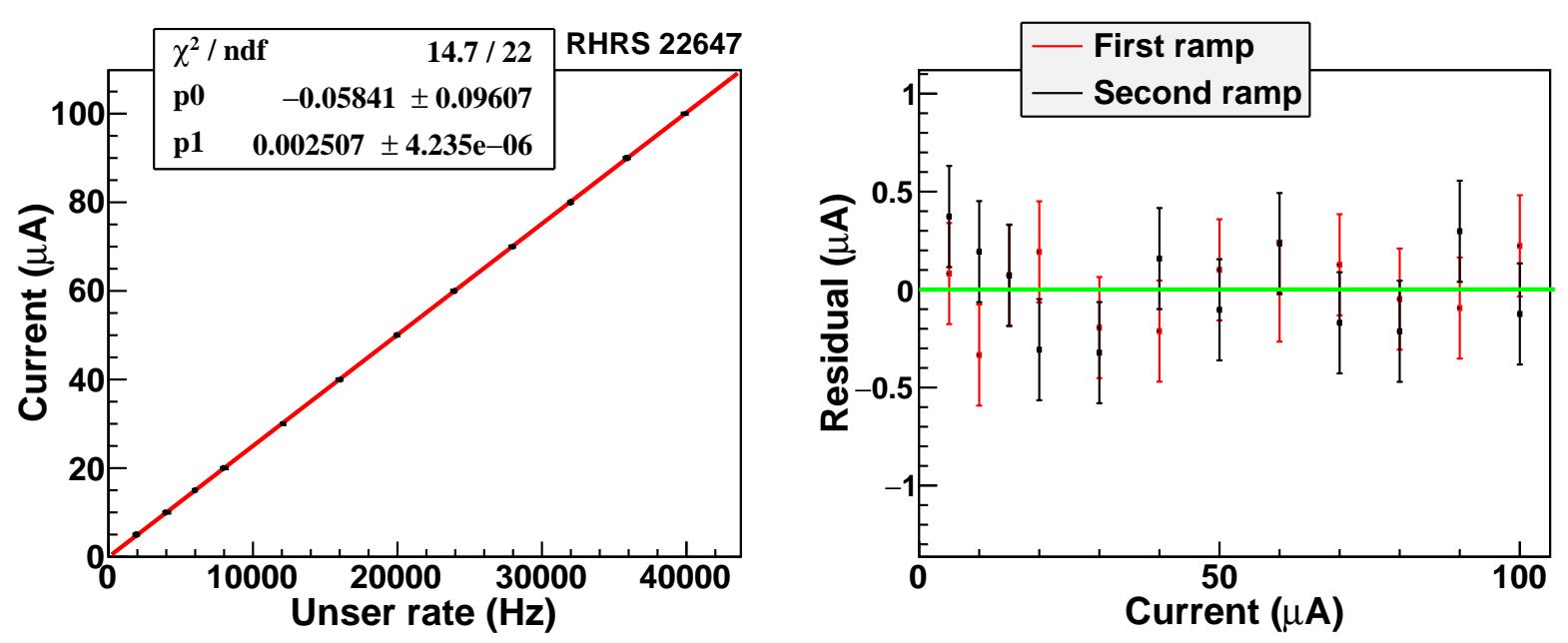

Figure 3.5. Left: The known injected current plotted as a function of offset subtracted Unser frequency. Right: The residual of the Unser current and the injected current source plotted, as a function of injected current.

and the set current from the precision current source. Here, $G$ is the Unser gain, $f_{\text {unser }}$ is average frequency for the current on period and $f_{0}$ is the corresponding average frequency for the current off period. The residuals show no dependence on current, indicating that the Unser is linear in the fitted region. The Unser calibrations were performed relatively frequently when the beam was off in order to study any possible gain stability. Table 3.1 lists the gains of Unser determined from different calibration run periods. The calibrations in Spring of 2016 show that the gain was stable at an average of $(2756 \pm 5.9) \times 10^{-6} \mu \mathrm{A} / \mathrm{Hz}$ whereas the calibrations during Fall of 2016 show that the gain was stable with an average of $(2505 \pm 4.6) \times 10^{-6} \mu \mathrm{A} / \mathrm{Hz}$. The change in the gain between these periods was the removal of a split of the Unser output going into the V-F for a parity experiment test.

\subsubsection{BCM Calibration}

As previously noted, the Unser has a very stable gain but has an offset that is both noisy and can drift over time. Therefore, it is not used for an integrated beam charge 
determination. In contrast, the BCMs have very stable offsets but their gain can drift over the time scale of weeks to months and need to be calibrated periodically against the Unser.

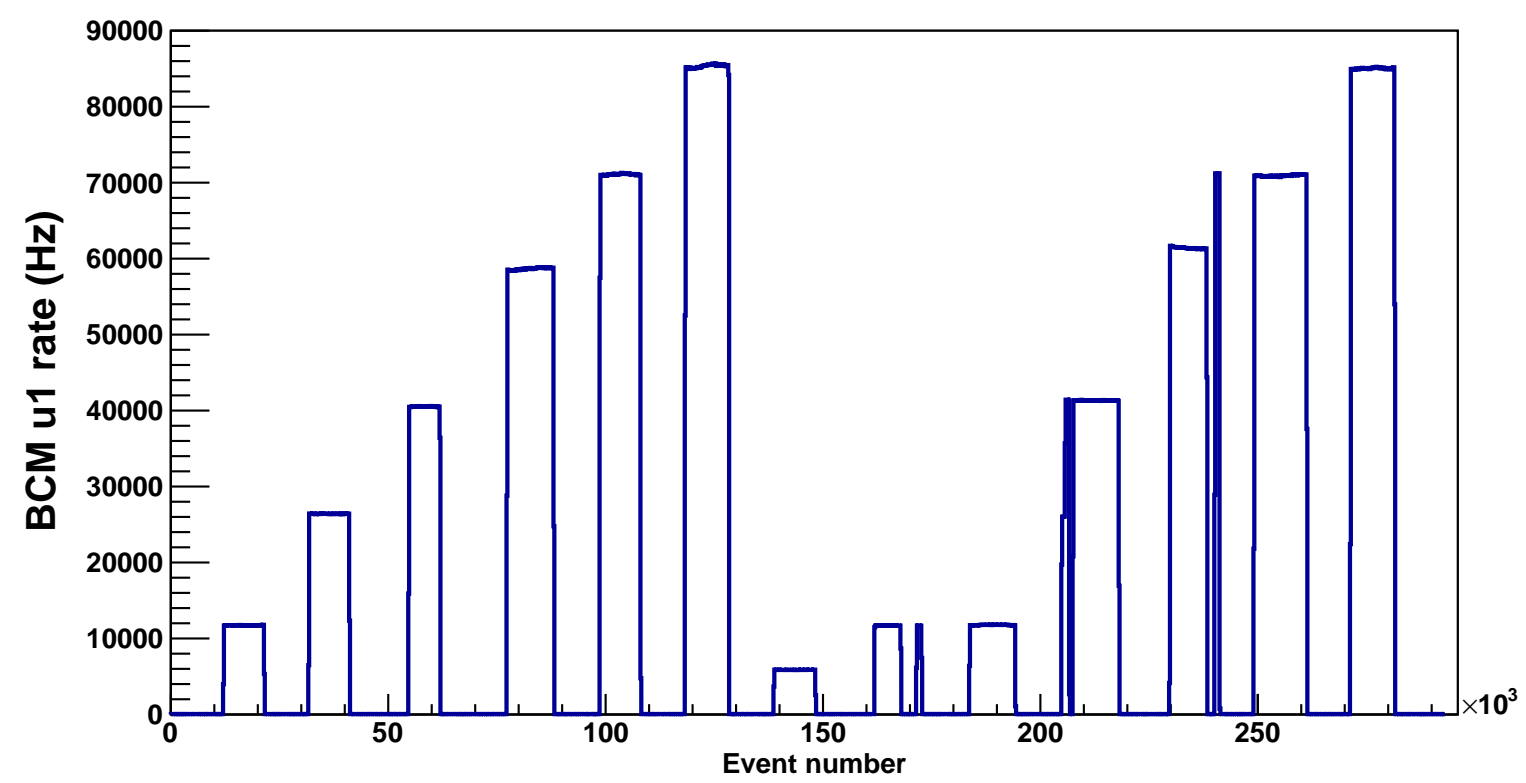

Figure 3.6. Figure shows an example plot of BCM u1 rate versus event number during BCM calibration. The time interval for each beam off and beam on period was 1 minute.

During a BCM calibration run the beam was turned on and off successively for alternating one minute periods while raising the current for each beam-on period. The BCM frequency versus event number is plotted in the Fig. 3.6. Receivers $\mathrm{u} 1$ and $\mathrm{d} 1$ are not expected to be linear below $10 \mu \mathrm{A}$, and the receiver $\mathrm{d} 3$ is only linear up to $60 \mu \mathrm{A}$ and $\mathrm{d} 10$ is expected to be linear only up to $30 \mu \mathrm{A}$ Therefore, the calibration fits were performed only over the current range where the receivers are highly linear.

During the calibration run, the BCM and Unser frequencies are stored in a scaler file to be used for the calibration. The Unser current is determined from Eq. 3.2 using the frequency $f$ determined from beam-on periods and the local $f_{0}$ determined from the adjacent beam-off periods. The plot in the upper left panel of Fig. 3.7 shows the fit to the current measured by the Unser as a function of the BCM u1 frequency. The offset and gain obtained are then used to convert scaler counts into electronic charge and the instantaneous beam current $\left(I_{\text {beam }}\right)$ is 

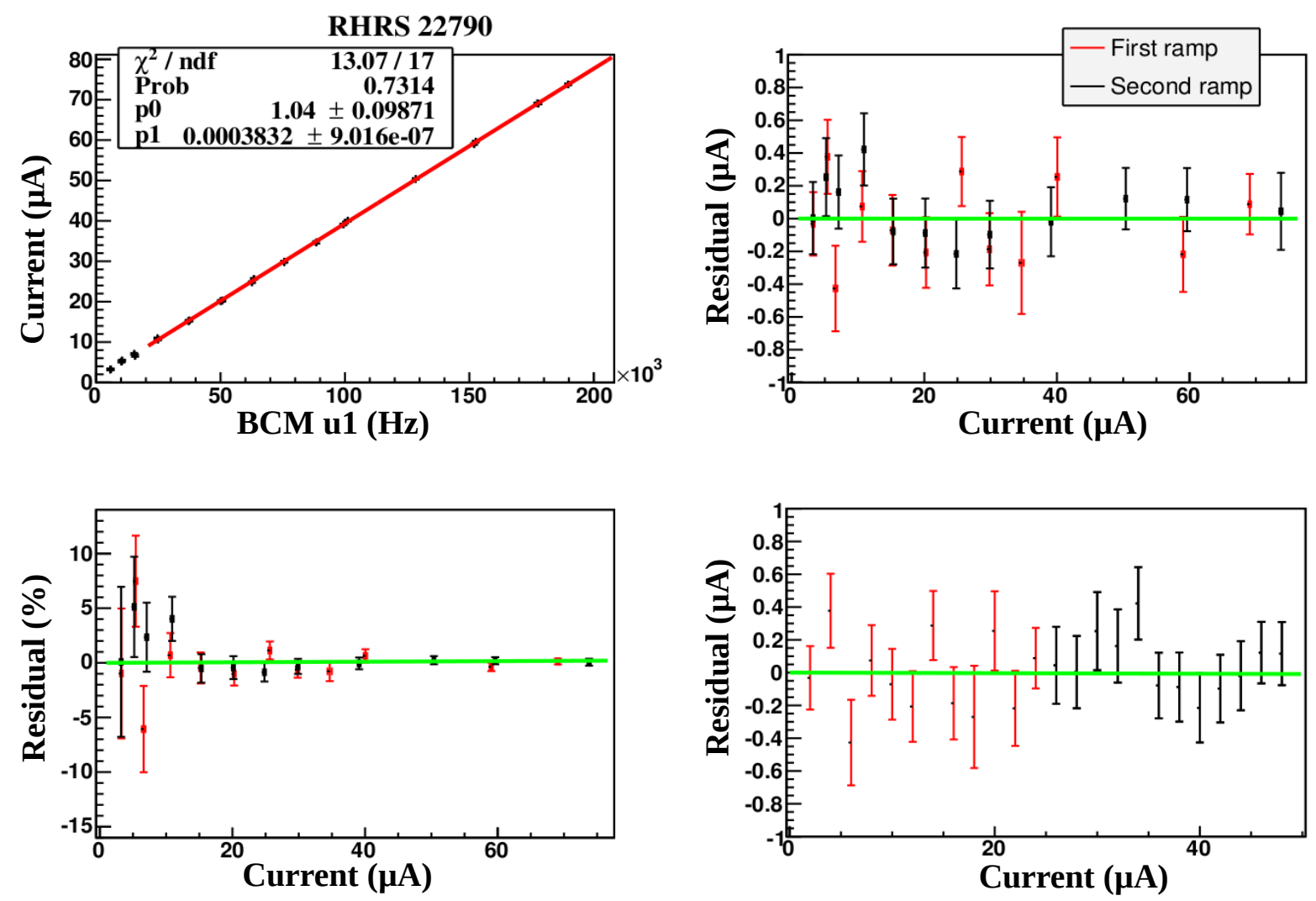

Figure 3.7. Top left panel shows the Unser current plotted against the BCM frequency. Top right shows the residual of the $\mathrm{BCM}$ current as given by $\mathrm{BCM}$ current obtained from the fit and the calculated Unser current plotted as a function BCM Current. Bottom left panel shows the percentage residual plotted against Unser current. The bottom right panel shows the residual versus time.

calculated by using the frequency of BCM in the working current range of each receiver as

$$
I_{B C M}=G_{B C M} \cdot f_{B C M}+I_{0},
$$

where, $O_{B C M}$ is the BCM offset. The total charge can be calculated by integrating the instantaneous beam current obtained from Eq. 3.3 as

$$
\Delta Q=\int I_{\text {beam }}(t) d t,
$$

The accumulated charge then 


$$
=\sum_{i}\left(G_{B C M} \times C+O_{B C M}\right) \times \Delta t_{i}
$$

where, $C$ is the total count from the scaler output and $\Delta t_{i}$ is the time separation between the successive scaler readings determined from the clock for which the current is above the threshold. The difference between the current determined from the BCM after calibration and the current measured by the Unser is shown in the top right panel of Fig. 3.7. It is seen that after calibration, the BCM u1 residuals are within $0.15 \%$ over the fitted current range. Determination of any offset within the uncertainty $0.1 \%$ is obtained from the study of target boiling of the solid target as discussed later in Section 3.4. Based on the stability study of the charge measurement using different receivers (as an example shown in Fig. 3.8 for receivers $u 1$ to $\mathrm{d} 1$ ), receiver $u 1$ was chosen as a default for the analysis. The uncorrelated uncertainty in the current measurement with this receiver was found to be $0.06 \mu \mathrm{A}$ based on the width of the distribution of the fit residuals, while calibration drift at $64 \mu \mathrm{A}$ was found to be $0.05 \mu \mathrm{A}$.

\subsubsection{Stability of Gain and Offsets}

Comparing calibration results performed at different points of time shows that the gains for the analog BCM receivers ( $\mathrm{u} 1, \mathrm{~d} 1, \mathrm{~d} 3$ and $\mathrm{d} 10)$ were stable within $1 \%$ whereas those for d10 are stable within (1-2)\% but the gain for new receivers changed twice: during a CHL (Central Helium Liquefier) shutdown (increased by factor of about 1.5), and after an IOC reboot, (decreased by factor of about 6 for Spring 2016 run period). In addition, the offset for the digital receivers was negative for some periods. For this reason the digital receivers were not used in this analysis. Fig. 3.9 shows the stability of gain and offsets for the analog BCMs receivers for Spring and Fall 2016 run periods. Receivers u1 and d1 were found to agree within $0.1 \%$ except for a few outliers. Tables 3.2 and 3.3 list the gains and offsets determined for the different BCM's calibrations performed in 2016. 


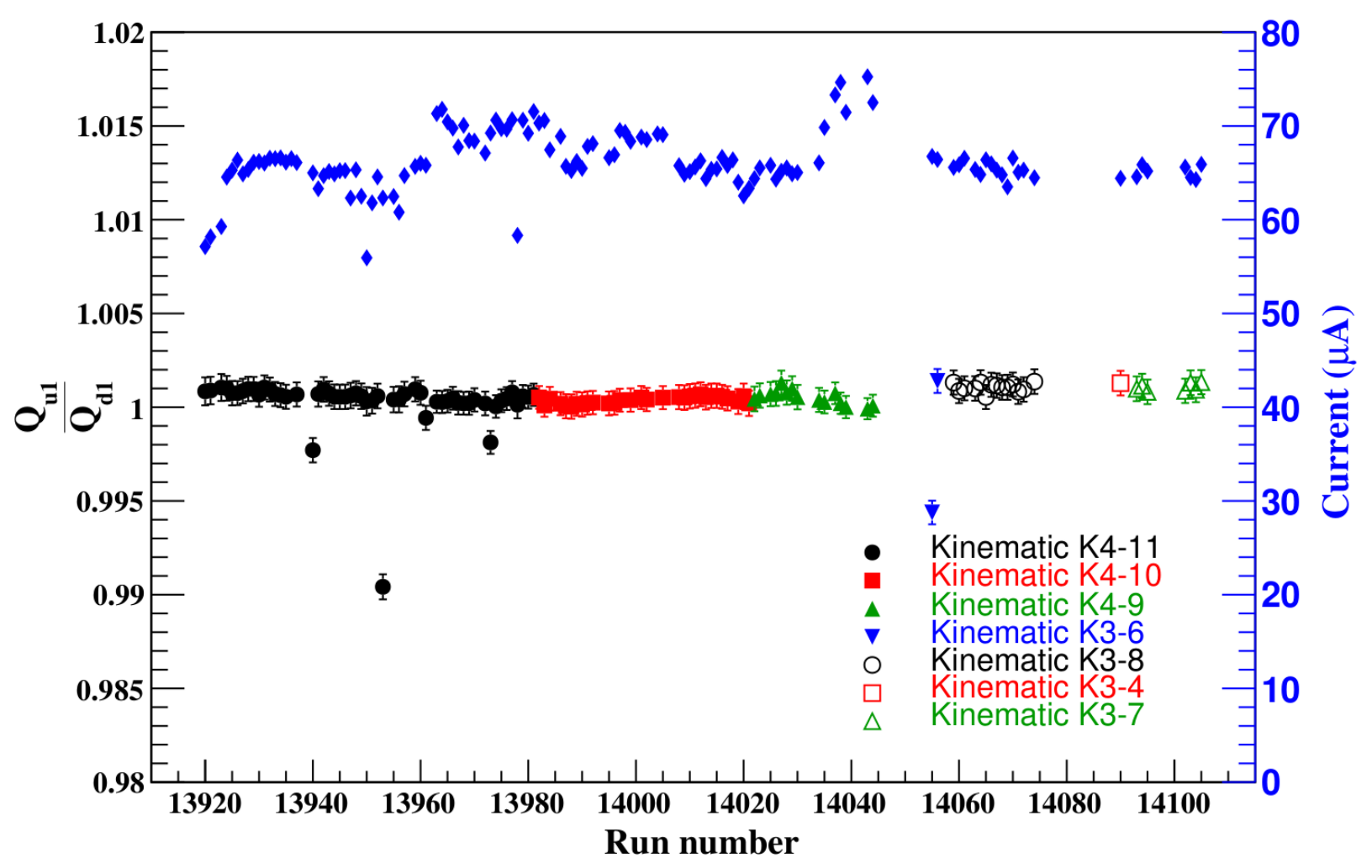

Figure 3.8. Charge accumulated by receiver u1 divided by charge accumulated by receiver d1 plotted against run number. The blue points are the average currents as indicated by the scale on the right.

\subsubsection{Global BCM Calibration}

The top plot in Fig. 3.10 shows the fit results for the u1 receiver when performing a global calibration utilizing all three Fall 2016 calibration runs. The top panel shows the Unser current fitted versus frequency of $\mathrm{u} 1$ receiver whereas, the middle plot shows the current residual as a function of Unser current, and the bottom panel shows the percentage residual as a function of Unser current for same period. The points from three different run periods are seen to be consistent with the residuals within $0.1 \%$ above $20 \mu \mathrm{A}$, consistent with the calibrations for this receiver. This again indicates that the gains and offsets from the individual calibrations for $\mathrm{u} 1$ are consistent. 
Table 3.2

Gains (in $\times 10^{-6} \mu \mathrm{A} / \mathrm{Hz}$ ) and offsets (in $\times 10^{-6} \mu \mathrm{A}$ ) for $\mathrm{u} 1$, d1 and d3 BCM receivers determined from different calibration runs.

\begin{tabular}{|c|c|c|c|c|c|c|c|}
\hline $\begin{array}{l}\text { Calib. } \\
\text { no. }\end{array}$ & $\begin{array}{l}\text { LHRS } \\
\text { Run }\end{array}$ & $\begin{array}{l}\text { Gain } u 1 \\
(\mu \mathrm{A} / \mathrm{Hz})\end{array}$ & $\begin{array}{l}\text { Offset } \mathrm{u} 1 \\
(\mu \mathrm{A})\end{array}$ & $\begin{array}{l}\text { Gain d1 } \\
(\mu \mathrm{A} / \mathrm{Hz})\end{array}$ & $\begin{array}{l}\text { Offset d1 } \\
(\mu \mathrm{A})\end{array}$ & $\begin{array}{l}\text { Gain d3 } \\
(\mu \mathrm{A} / \mathrm{Hz})\end{array}$ & $\begin{array}{l}\text { Offset d3 } \\
(\mu \mathrm{A})\end{array}$ \\
\hline 1 & 12382 & $354.0 \pm 3.7$ & $0.8 \pm 0.2$ & $321.7 \pm 3.4$ & $0.7 \pm 0.2$ & $82.7 \pm 1.0$ & $0.9 \pm 0.2$ \\
\hline 2 & 12514 & $344.3 \pm 3.5$ & $0.6 \pm 0.3$ & $322.3 \pm 3.2$ & $0.6 \pm 0.2$ & $93.9 \pm 5.7$ & $0.2 \pm 0.1$ \\
\hline 3 & 12916 & $347.5 \pm 2.7$ & $1.1 \pm 0.1$ & $316.3 \pm 2.4$ & $1.0 \pm 0.1$ & $92.9 \pm 0.5$ & $0.4 \pm 0.1$ \\
\hline 4 & 13220 & $352.9 \pm 2.1$ & $0.7 \pm 0.2$ & $320.2 \pm 1.9$ & $0.4 \pm 0.2$ & $93.4 \pm 0.7$ & $0.3 \pm 0.1$ \\
\hline 5 & 13447 & $352.5 \pm 0.9$ & $0.5 \pm 0.1$ & $320.8 \pm 0.9$ & $0.1 \pm 0.1$ & $92.8 \pm 0.2$ & $0.2 \pm 0.1$ \\
\hline 6 & 13852 & $384.4 \pm 0.8$ & $1.1 \pm 0.1$ & $329.2 \pm 0.7$ & $0.5 \pm 0.1$ & $95.5 \pm 0.2$ & $0.4 \pm 0.1$ \\
\hline 7 & 14252 & $383.1 \pm 3.4$ & $1.3 \pm 0.2$ & $326.3 \pm 2.9$ & $0.8 \pm 0.2$ & $97.7 \pm 0.6$ & $0.1 \pm 0.1$ \\
\hline 8 & 14545 & $388.6 \pm 2.2$ & $0.8 \pm 0.2$ & $331.7 \pm 2.1$ & $0.3 \pm 0.2$ & $96.8 \pm 0.4$ & $0.2 \pm 0.1$ \\
\hline 9 & Global & $384.8 \pm 1.7$ & $1.1 \pm 0.1$ & $328.8 \pm 1.6$ & $0.6 \pm 0.1$ & $97.1 \pm 0.3$ & $0.2 \pm 0.1$ \\
\hline $\begin{array}{l}\text { Calib. } \\
\text { no. }\end{array}$ & $\begin{array}{l}\text { RHRS } \\
\text { Run }\end{array}$ & $\begin{array}{l}\text { Gain u1 } \\
(\mu \mathrm{A} / \mathrm{Hz})\end{array}$ & $\begin{array}{l}\text { Offset } u 1 \\
(\mu \mathrm{A})\end{array}$ & $\begin{array}{l}\text { Gain d1 } \\
(\mu \mathrm{A} / \mathrm{Hz})\end{array}$ & $\begin{array}{l}\text { Offset d1 } \\
(\mu \mathrm{A})\end{array}$ & $\begin{array}{l}\text { Gain d3 } \\
(\mu \mathrm{A} / \mathrm{Hz})\end{array}$ & $\begin{array}{l}\text { Offset d3 } \\
(\mu \mathrm{A})\end{array}$ \\
\hline 1 & 21731 & $346.7 \pm 4.30$ & $1.1 \pm 0.2$ & $315.7 \pm 3.9$ & $0.8 \pm 0.3$ & $93.8 \pm 0.9$ & $0.3 \pm 0.2$ \\
\hline 2 & 21807 & $345.0 \pm 2.9$ & $0.9 \pm 0.2$ & $315.1 \pm 2.6$ & $0.6 \pm 0.2$ & $93.1 \pm 0.7$ & $0.3 \pm 0.2$ \\
\hline 3 & 22097 & $342.2 \pm 2.9$ & $1.2 \pm 0.1$ & $312.6 \pm 2.6$ & $0.8 \pm 0.1$ & $92.5 \pm 0.6$ & $0.4 \pm 0.1$ \\
\hline 4 & 22298 & $350.2 \pm 2.8$ & $0.8 \pm 0.2$ & $318.9 \pm 2.3$ & $0.4 \pm 0.2$ & $93.4 \pm 0.6$ & $0.3 \pm 0.2$ \\
\hline 5 & 22476 & $348.7 \pm 0.9$ & $0.7 \pm 0.1$ & $319.1 \pm 0.9$ & $0.2 \pm 0.1$ & $92.7 \pm 0.2$ & $0.2 \pm 0.1$ \\
\hline 6 & 22790 & $383.2 \pm 0.9$ & $1.0 \pm 0.1$ & $328.0 \pm 0.8$ & $0.5 \pm 0.1$ & $95.5 \pm 0.2$ & $0.4 \pm 0.1$ \\
\hline 7 & 23071 & $381.0 \pm 3.9$ & $1.3 \pm 0.2$ & $324.7 \pm 3.2$ & $0.9 \pm 0.2$ & $97.3 \pm 0.6$ & $0.2 \pm 0.1$ \\
\hline 8 & 23280 & $388.5 \pm 3.8$ & $0.7 \pm 0.3$ & $332.7 \pm 3.3$ & $0.2 \pm 0.3$ & $0.9 \pm 0.6$ & $0.1 \pm 0.2$ \\
\hline 9 & Global & $382.4 \pm 0.9$ & $1.2 \pm 0.1$ & $327.0 \pm 0.7$ & $0.6 \pm 0.1$ & $96.0 \pm 0.2$ & $0.4 \pm 0.1$ \\
\hline
\end{tabular}


Table 3.3

Gains (in $\times 10^{-6} \mu \mathrm{A} / \mathrm{Hz}$ ) and offsets (in $\times 10^{-6} \mu \mathrm{A}$ ) for $\mathrm{d} 10$, unew and dnew BCM receivers determined from different calibration runs. Calibrations 1-5 were taken in Spring 2016 and 6-8 were taken in Fall 2016

\begin{tabular}{|c|c|c|c|c|c|c|c|}
\hline $\begin{array}{l}\text { Calib. } \\
\text { no. }\end{array}$ & $\begin{array}{l}\text { LHRS } \\
\text { Run }\end{array}$ & $\begin{array}{l}\text { Gain d10 } \\
(\mu \mathrm{A} / \mathrm{Hz})\end{array}$ & $\begin{array}{l}\text { Offset } \\
\mathrm{d} 10(\mu \mathrm{A})\end{array}$ & $\begin{array}{l}\text { Gain } \\
\text { unew } \\
(\mu \mathrm{A} / \mathrm{Hz})\end{array}$ & $\begin{array}{l}\text { Offset } \\
\text { unew } \\
(\mu \mathrm{A})\end{array}$ & $\begin{array}{l}\text { Gain } \\
\text { dnew } \\
(\mu \mathrm{A} / \mathrm{Hz})\end{array}$ & $\begin{array}{l}\text { Offset } \\
\text { dnew } \\
(\mu \mathrm{A})\end{array}$ \\
\hline 1 & 12382 & $32.8 \pm 0.4$ & $0.1 \pm 0.2$ & $N A$ & $N A$ & $N A$ & $N A$ \\
\hline 2 & 12514 & $32.2 \pm 0.3$ & $0.1 \pm 0.1$ & $200.7 \pm 1.2$ & $0.0 \pm 0.1$ & $172.9 \pm 1.3$ & $0.1 \pm 0.1$ \\
\hline 3 & 13220 & $32.1 \pm 0.5$ & $0.2 \pm 0.2$ & $294.3 \pm 1.4$ & $0.3 \pm 0.1$ & $249.0 \pm 1.6$ & $0.2 \pm 0.1$ \\
\hline 4 & 13447 & $31.9 \pm 0.7$ & $0.1 \pm 0.2$ & $50.0 \pm 0.3$ & $0.1 \pm 0.1$ & $42.9 \pm 0.3$ & $0.0 \pm 0.1$ \\
\hline 5 & 13852 & $32.9 \pm 0.3$ & $0.1 \pm 0.2$ & $254.6 \pm 0.5$ & $0.2 \pm 0.1$ & $215.5 \pm 0.4$ & $0.1 \pm 0.1$ \\
\hline 6 & 14252 & $34.4 \pm 0.3$ & $-0.1 \pm 0.1$ & $258.1 \pm 1.6$ & $0.0 \pm 0.1$ & $223.6 \pm 1.4$ & $-0.0 \pm 0.1$ \\
\hline 7 & 14545 & $33.1 \pm 0.3$ & $0.1 \pm 0.1$ & $254.9 \pm 1.0$ & $0.0 \pm 0.1$ & $224.4 \pm 0.9$ & $0.0 \pm 0.1$ \\
\hline 8 & 10505 & $33.1 \pm 0.3$ & $0.1 \pm 0$. & $254.9 \pm 1.0$ & $0.0 \pm 0.1$ & $224.4 \pm 0.9$ & $0.0 \pm 0.1$ \\
\hline 9 & Global & $33.7 \pm 0.2$ & $0.0 \pm 0.1$ & $255.5 \pm 0.9$ & $0.1 \pm 0.1$ & $224.2 \pm 0.7$ & $-0.0 \pm 0.1$ \\
\hline $\begin{array}{l}\text { Calib. } \\
\text { no. }\end{array}$ & $\begin{array}{l}\text { RHRS } \\
\text { Run }\end{array}$ & $\begin{array}{l}\text { Gain } \\
\mathrm{d} 10(\mu \mathrm{A} / \mathrm{Hz})\end{array}$ & $\begin{array}{l}\text { Offset } \\
\mathrm{d} 10(\mu \mathrm{A})\end{array}$ & $\begin{array}{l}\text { Gain } \\
\text { unew } \\
(\mu \mathrm{A} / \mathrm{Hz})\end{array}$ & $\begin{array}{l}\text { Offset } \\
\text { unew } \\
(\mu \mathrm{A})\end{array}$ & $\begin{array}{l}\text { Gain } \\
\text { dnew } \\
(\mu \mathrm{A} / \mathrm{Hz})\end{array}$ & $\begin{array}{l}\text { Offset } \\
\text { dnew } \\
(\mu \mathrm{A})\end{array}$ \\
\hline 1 & 21731 & $32.5 \pm 0.5$ & $0.2 \pm 0.2$ & $N A$ & $N A$ & $N A$ & $N A$ \\
\hline 2 & 21807 & $32.2 \pm 0.4$ & $0.2 \pm 0.2$ & $199.1 \pm 1.5$ & $0.1 \pm 0.2$ & $171.9 \pm 1.3$ & $0.1 \pm 0.2$ \\
\hline 3 & 22097 & $3.3 \pm 0.4$ & $0.2 \pm 0.2$ & $199.1 \pm 1.3$ & $0.3 \pm 0.1$ & $172.1 \pm 1.1$ & $0.2 \pm 0.1$ \\
\hline 4 & 22298 & $31.7 \pm 0.6$ & $0.3 \pm 0.2$ & $294.1 \pm 1.5$ & $0.2 \pm 0.1$ & $248.8 \pm 1.2$ & $0.1 \pm 0.2$ \\
\hline 5 & 22476 & $31.4 \pm 0.3$ & $0.3 \pm 0.1$ & $50.0 \pm 0.3$ & $0.1 \pm 0.1$ & $42.9 \pm 0.3$ & $0.0 \pm 0.1$ \\
\hline 6 & 22790 & $33.2 \pm 0.3$ & $0.2 \pm 0.1$ & $254.7 \pm 0.5$ & $0.1 \pm 0.1$ & $215.4 \pm 0.4$ & $0.1 \pm 0.1$ \\
\hline 7 & 23071 & $34.2 \pm 0.3$ & $-0.0 \pm 0.1$ & $256.9 \pm 1.7$ & $0.1 \pm 0.1$ & $222.6 \pm 1.4$ & $0.1 \pm 0.1$ \\
\hline 8 & 23280 & $32.9 \pm 0.5$ & $0.1 \pm 0.2$ & $255.5 \pm 1.7$ & $-0.1 \pm 0.2$ & $224.7 \pm 1.5$ & $0.1 \pm 0.1$ \\
\hline 9 & Global & $33.4 \pm 0.2$ & $0.1 \pm 0.1$ & $254.6 \pm 0.4$ & $0.1 \pm 0.1$ & $N A$ & $N A$ \\
\hline
\end{tabular}



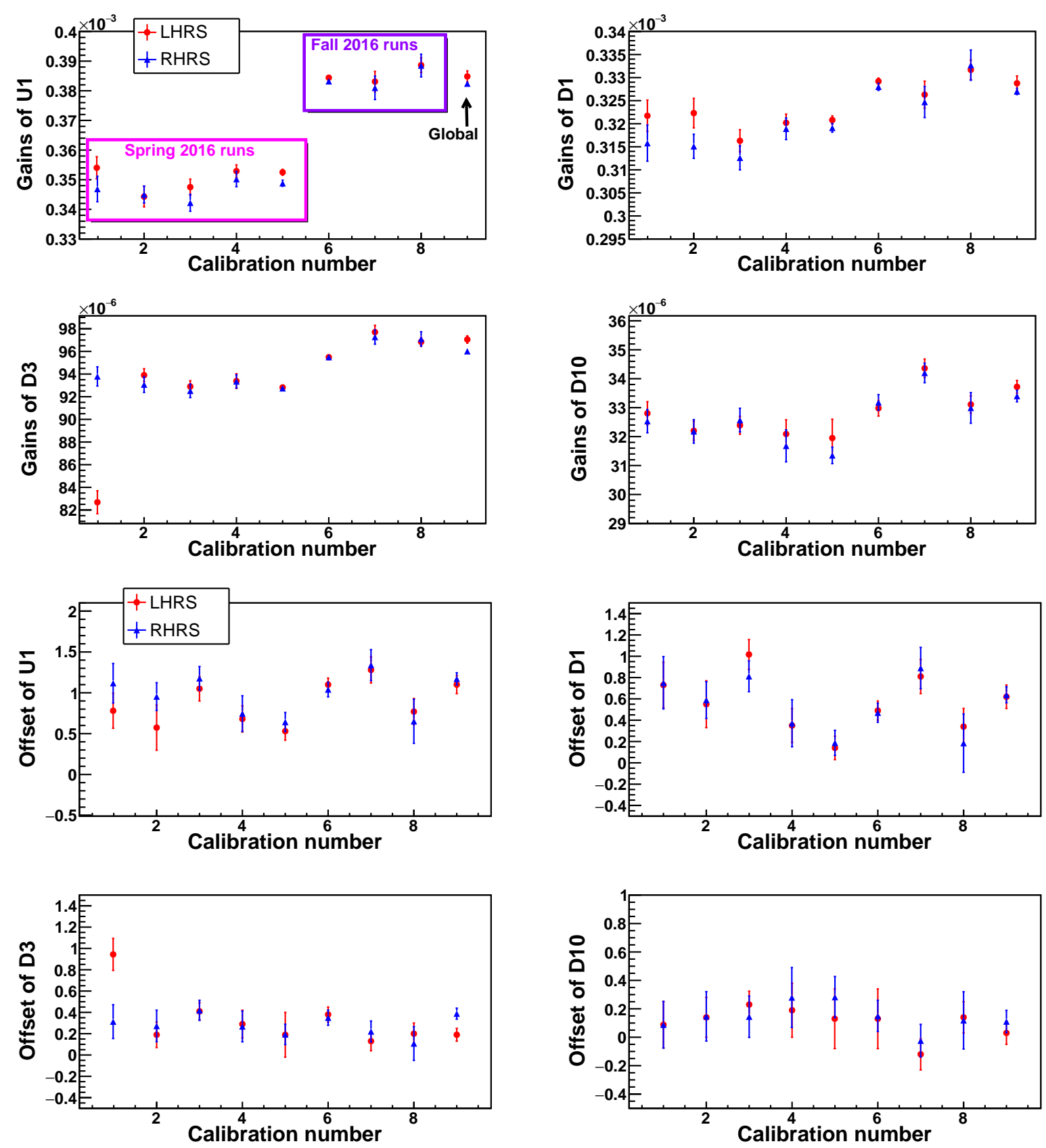

Figure 3.9. BCM Gains and offsets versus calibration run number showing the stability during the experiment. Calibration number 1 through 5 are from Spring of 2016, while calibration numbers 6 through 8 are from the Fall of 2016 and the calibration number 8 is the global as noted on the upper left panel. 

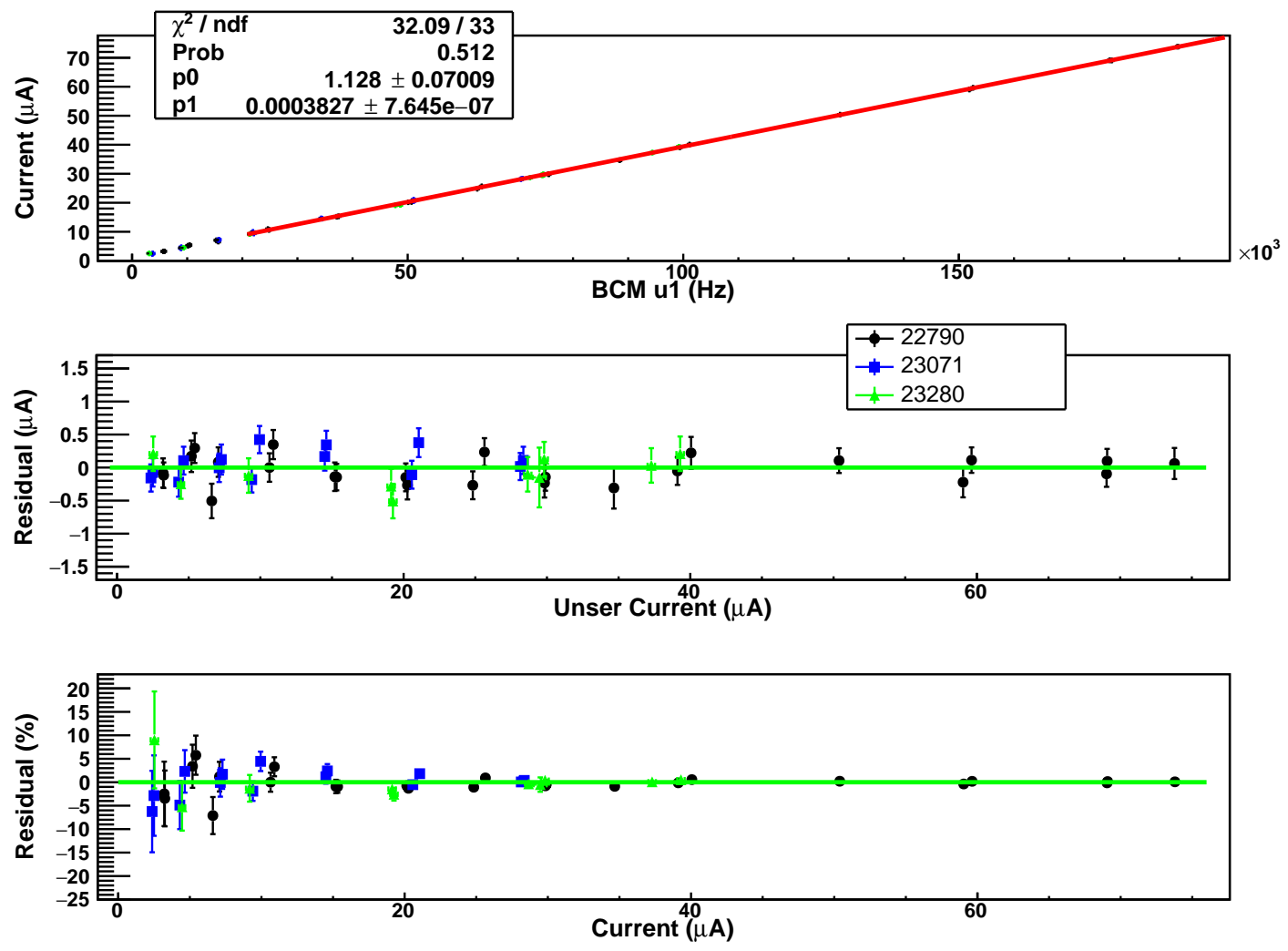

Figure 3.10. Fall 2016 global BCM global calibration results for receiver u1. Top left panel shows the Unser current plotted against the BCM frequency. Top right shows the residual of the BCM current as given by BCM current obtained from the fit and the calculated Unser current plotted as a function BCM Current. Bottom left panel shows the percentage residual plotted against Unser current. The bottom right panel shows the residual versus time. 


\subsection{Target Boiling Study}

When an electron beam passes through the target it can deposit a significant amount of energy (about $5 \mathrm{~W}$ per $\mu \mathrm{A}$ ) in the form of heat, which can cause boiling of the cryogen and create a local reduction in the target density. In order to study the effect of boiling on the Liquid Hydrogen (LH2) target, runs were taken over a range of currents using the $15 \mathrm{~cm}$ LH2 target (in Loop2 during Fall 2016 and in Loop3 during Spring 2016). Additionally, the central carbon target was utilized to separate any possible rate systematic from boiling, since this solid target should show no boiling. Additionally, any dependence of the normalized yield on the beam current from this target is then utilized to determine any absolute current offset from the BCM calibration. A range of $3-67 \mu \mathrm{A}$ beam current and a $2 \times 2 \mathrm{~mm}^{2}$ raster was used for this study. The density reduction should increase with current, wheres it should decrease with raster size. For the target boiling studies two types of analysis have been made:

- a scaler analysis in which the normalized scaler rate (scaler rate divided by current) is fitted against beam current, with

$$
\text { Normalized scaler rate }=\frac{\text { Trigger } 1 \text { rate }}{\text { Beam Current }}
$$

- and a yield analysis in which the normalized yield for good electron events determined by PID and single cluster cuts normalized to the effective beam charge, after correcting for efficiencies and live time with

$$
\text { Normalized Yield }=\frac{\text { No. of events } \times \text { PS }}{\text { Charge } \times \text { efficiency } \times \text { Live time }}
$$

The efficiency correction is the product of PID cut efficiency, tracking and trigger efficiencies. i.e.

$$
\text { Efficiency }(\epsilon)=\epsilon_{\text {tracking }} \times \epsilon_{P I D} \times \epsilon_{\text {trigger }} \text {. }
$$


Computer dead time arises when the DAQ is unable to process every event from every trigger. Both electronic as well as computer dead time can affect the normalized yield as discussed later in the section 3.7 .

Even though the scaler analysis is quicker and easier to analyse, this method does not have any selection to get rid of possible events originating from the beamline due to beam halo striking the beam pipe downstream of the target and producing scattered electrons reaching to the detector but not going through the magnetic elements.

\subsubsection{Selection of Good Events for Yield Analysis}

In order to select good events the following cuts were used:

1. Beam trip cut: Only events collected during the periods for which beam current was stable and not ramping, and with the total accumulated charge between the scaler reads for those periods as shown in the Fig 3.11.

2. Particle identification cuts: These cuts were use to select electrons from the sample, and includes a cut in number of photoelectrons in the Cherenkov and total energy deposited in the calorimeter normalized to the particle momentum as shown in Fig. 3.12 which will be discussed in the section 3.5 .

3. Single cluster cuts: These cuts were applied to select good tracks in the VDC. This requirement is that only a single cluster exists in each of the four VDC planes, for each selected event. This cut eliminates bad tracks due to ambiguous clusters in the track information as discussed later in Section 3.5.

\subsubsection{Preliminary Results of Target Boiling Study}

In this section the preliminary study of target boiling prior to the final detector calibration will be presented to outline the procedure that was performed for this dissertation. The 
Beam trip cut

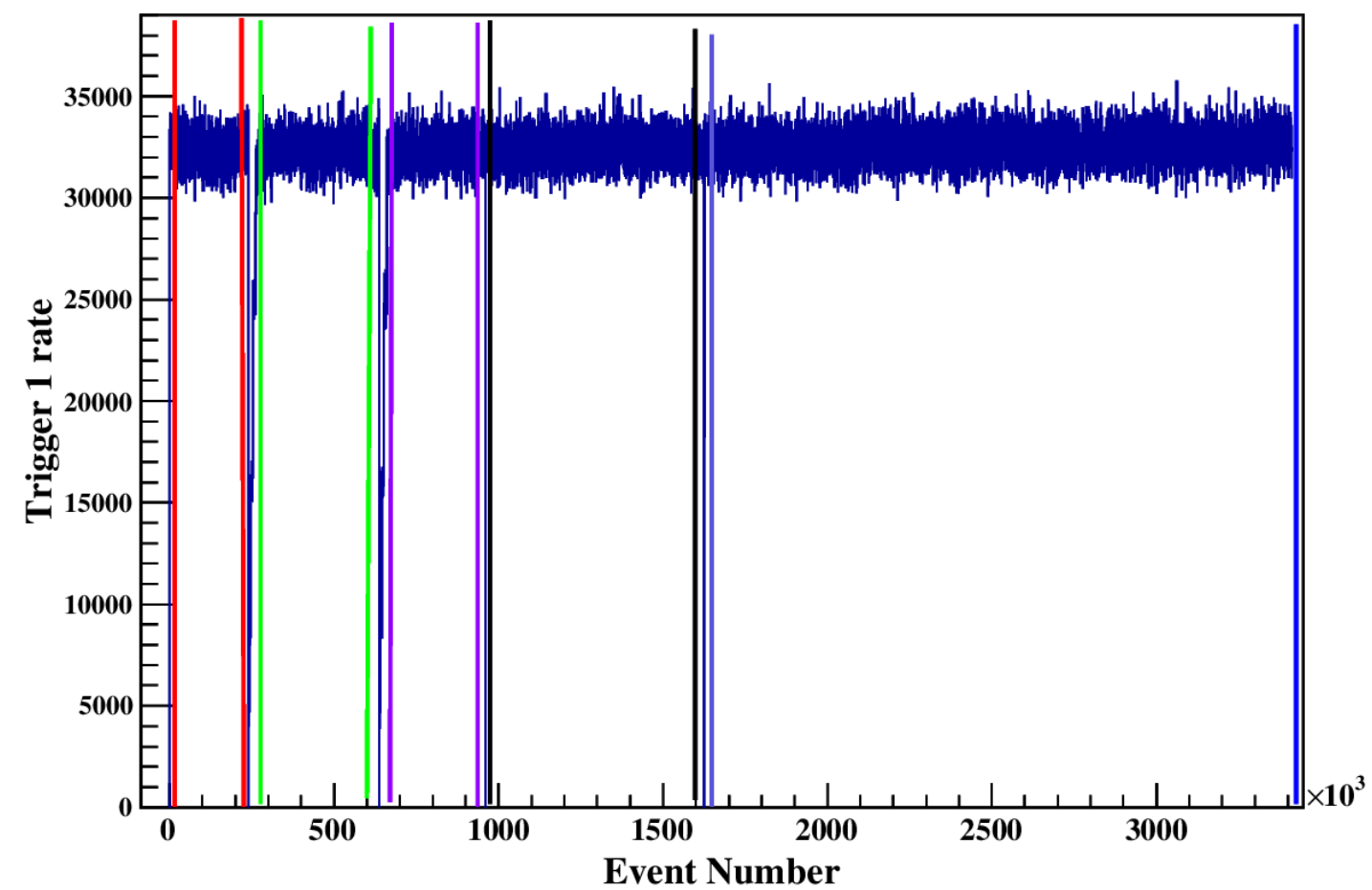

Figure 3.11. The rate for Trigger 1 versus entry number used to select entries when the beam was on. The region between the vertical line of a particular color denote the region of good entries when current was stable and not ramping.

results after the final detector calibration were performed by B. Schmookler based on this procedure [13]. As shown in Fig. 3.13 the top panel shows the charge normalized yields plotted as a function of current and on the bottom panel shows the normalized rates plotted as a function of current for both LH2 (red) and Carbon (blue).

\subsubsection{Offset in Measured Current}

To determined a possible current offset, the normalized rate from the carbon scaler analysis are fitted using the function expected of

$$
F(I)=\frac{a_{o}}{1+\Delta I / I} .
$$




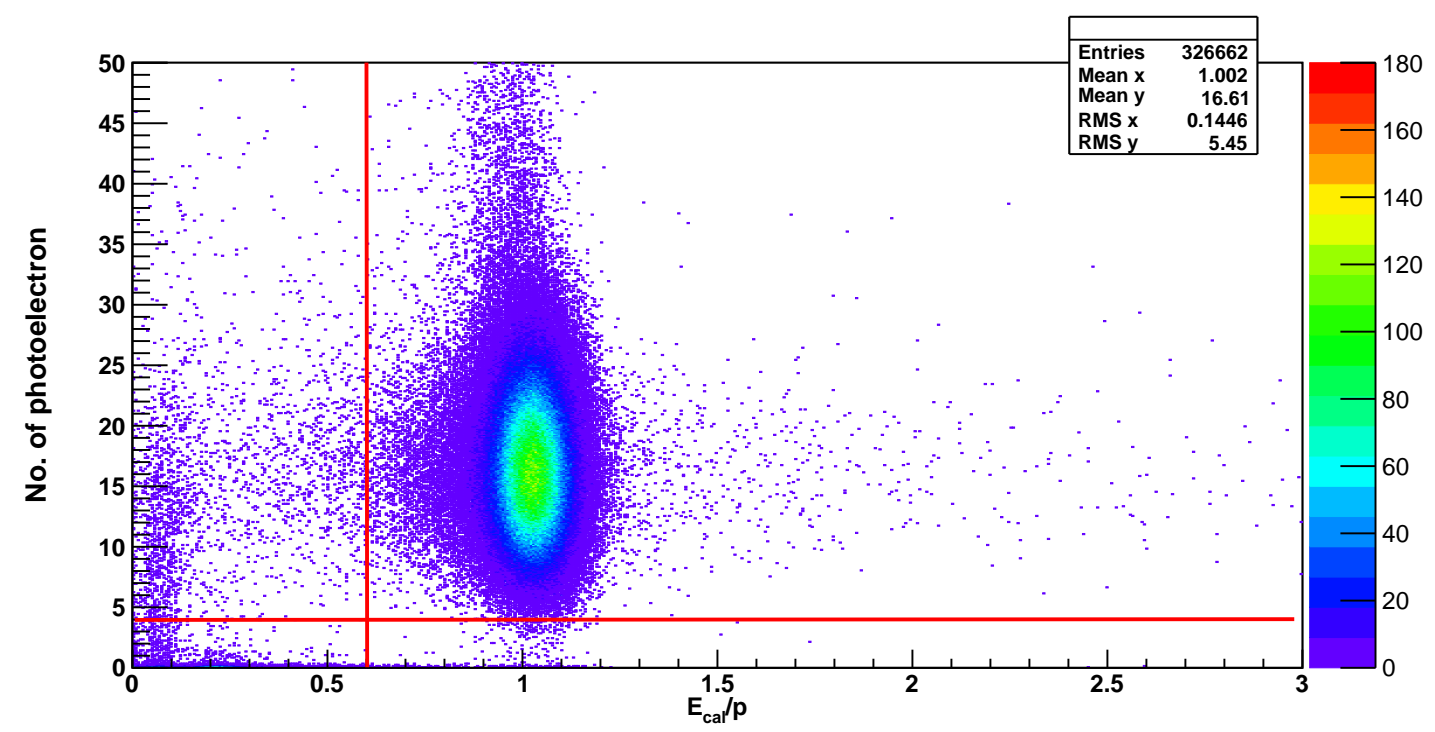

Figure 3.12. The number of photoelectrons in Cherenkov as a function of energy deposited in the calorimeter normalized by momentum showing the selection cuts for good electrons. Everything to the right of the vertical red line in $E_{c a l} / p$ and above the horizontal red line in Cherenkov number of photoelectrons represents the good electron events.

The fit is shown in Fig. 3.14, where $\Delta I$ gives the current offset. From this analysis, the value of the current offset was found to be $0.128 \mu \mathrm{A}$, and was subtracted from beam current and the integrated charge. After this correction the normalized yields and normalized rates are plotted as a function of corrected beam currents as shown in the Fig. 3.15. Clearly, after applying the correction to the offset, the carbon shows no boiling within the uncertainty, whereas hydrogen shows a boiling of $(-2.7 \pm 0.39) \%$ per $100 \mu \mathrm{A}$ from the scaler analysis. The results of yield analysis after improvement to PID detectors calibrations are shown in Fig. 3.18 performed by B. Schmookler [13]. In addition to this, beta cut were included to remove cosmic events. 

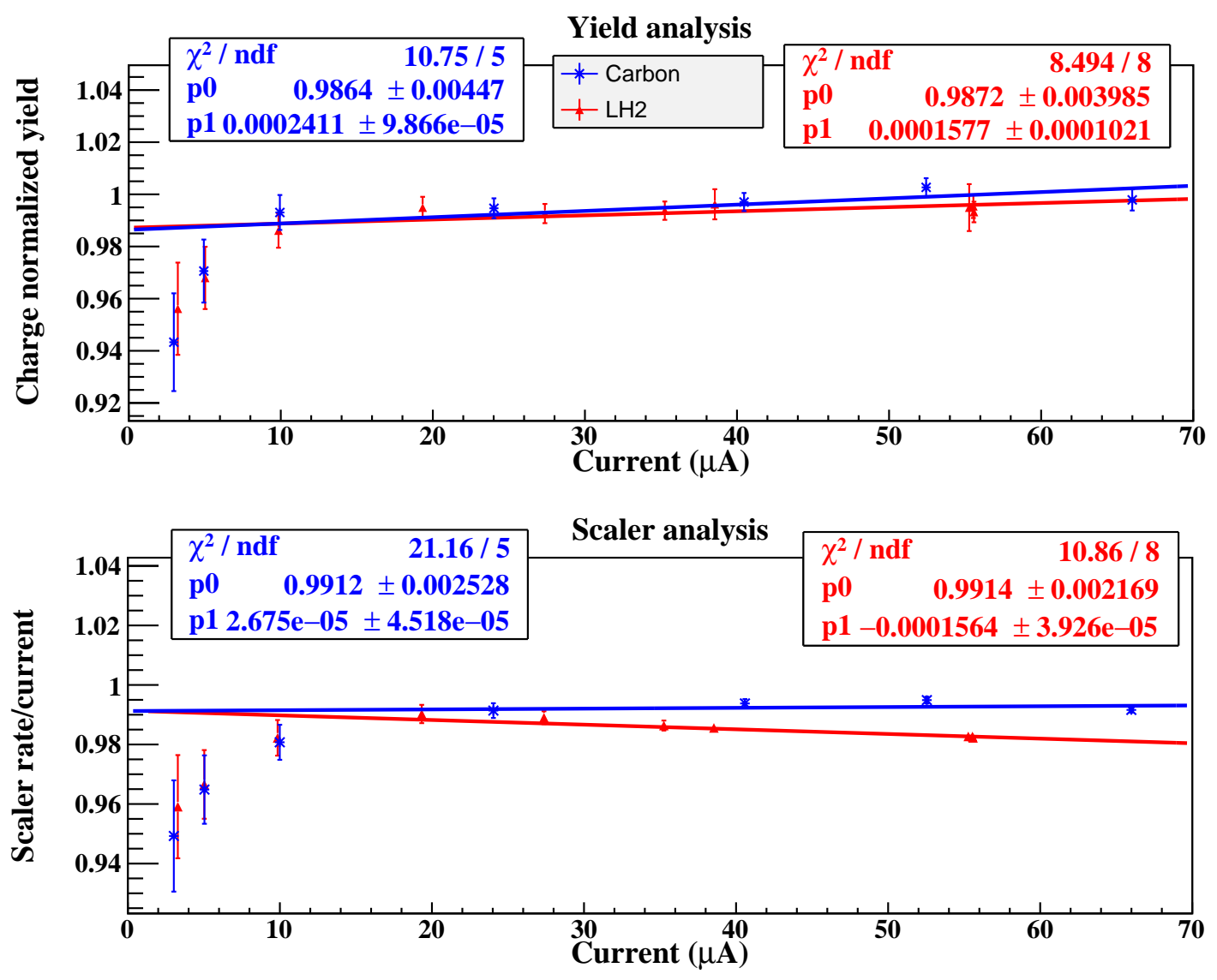

Figure 3.13. Preliminary results of boiling studies on carbon (blue) and LH2 (red) targets. The top plot is showing charge normalized yield vs beam current and the bottom plot is showing scaler rate vs beam current prior to being corrected for the measured offset current. 


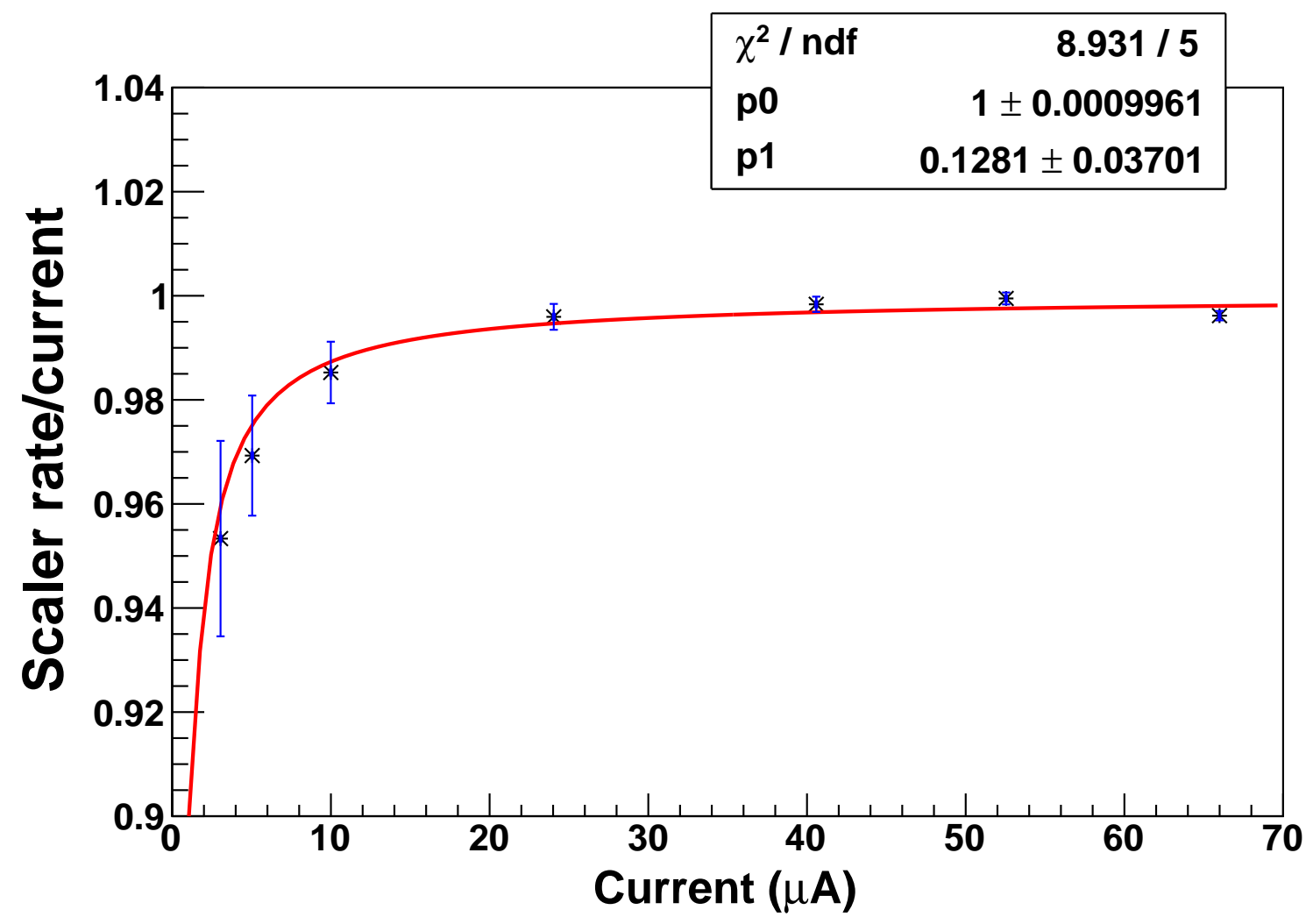

Figure 3.14. Scaler rate divided by current for T1 trigger as a function of beam current for carbon target. The systematic shape expected for an absolute current offset is fit to the data (given by red line) and yields a current offset of $0.13 \mu \mathrm{A}$. 

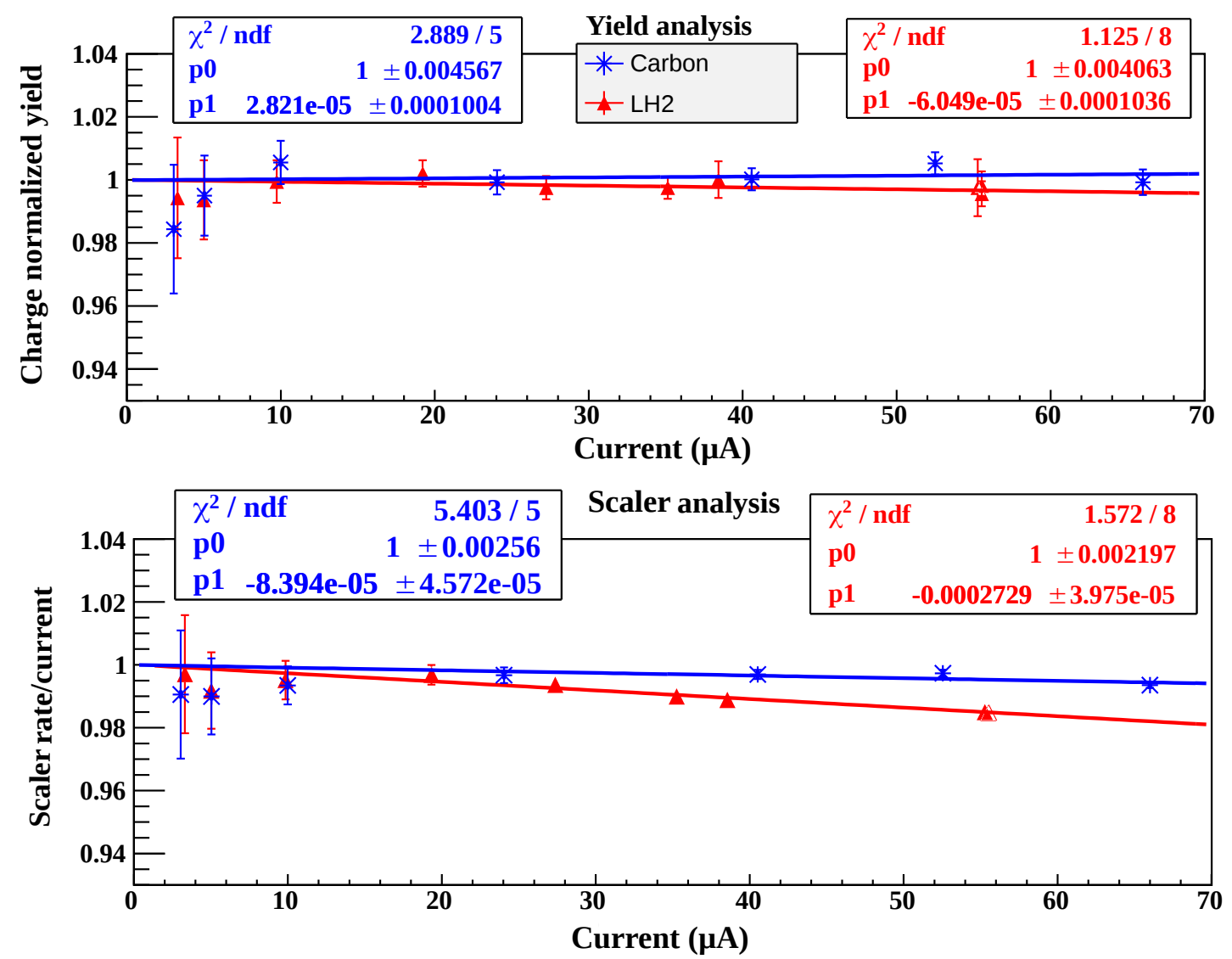

Figure 3.15. Results of boiling studies on carbon (blue) and LH2 (red) targets, after correcting for the offset in beam current. 

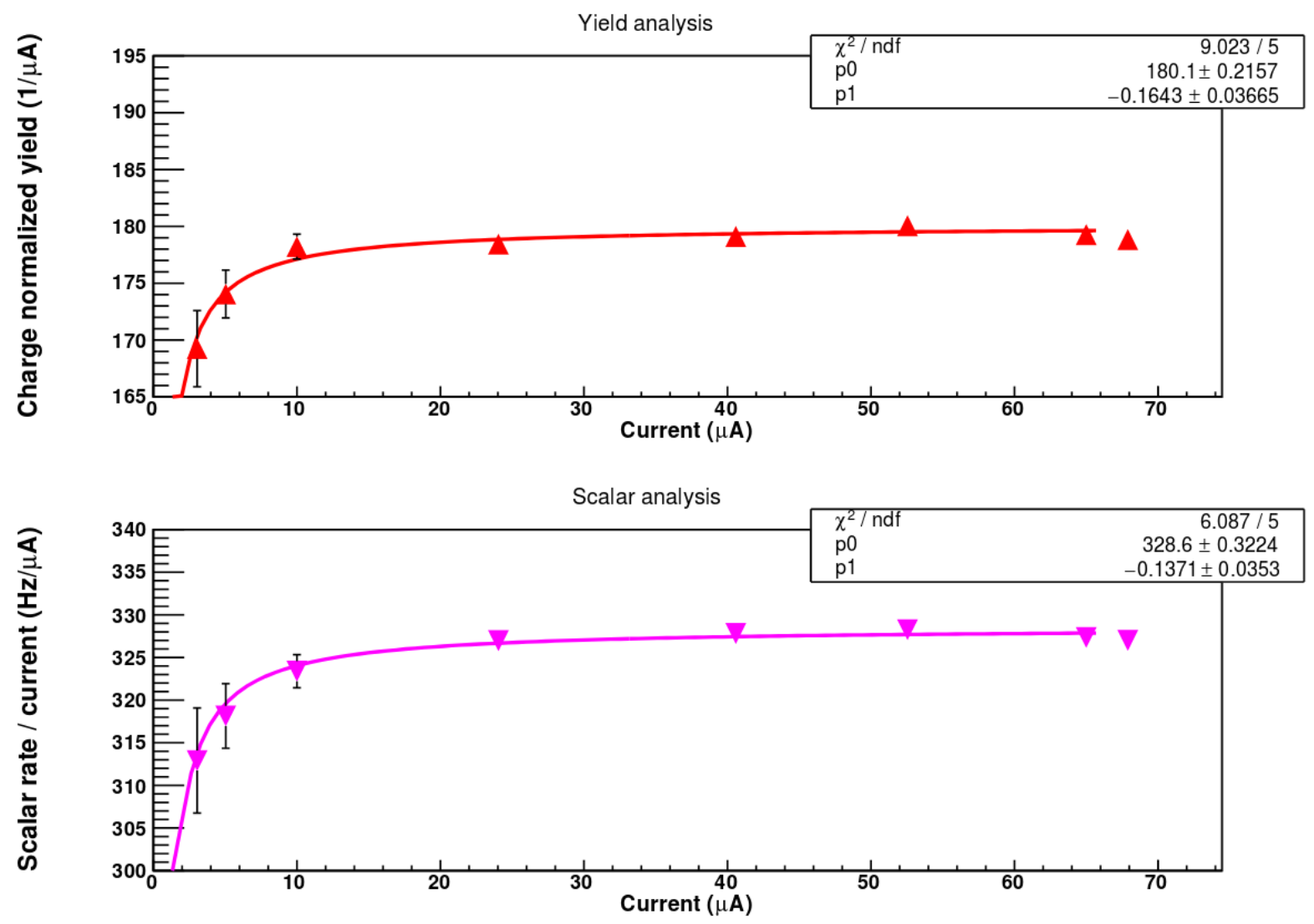

Figure 3.16. Charge normalized yield (top) and scaler rate normalized to current (bottom) versus beam current and fit to absolute current offset using the fit as discussed in Eq. 3.9 for the carbon target from the final analysis of B. Schmookler [13]. 

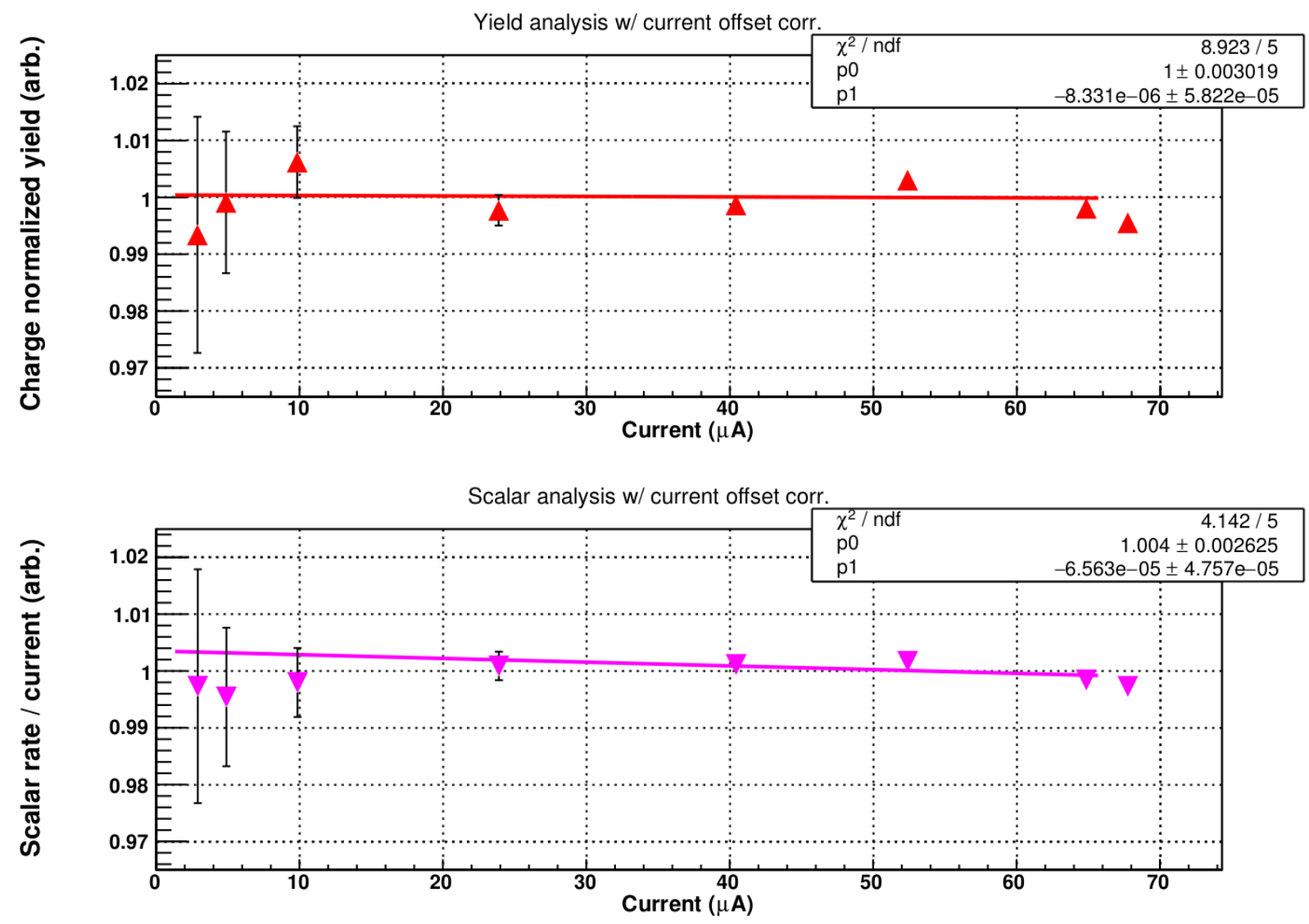

Figure 3.17. The final results of boiling study carbon target showing both yield (top) and scaler (bottom) analysis [13] after correcting for the current offset. 

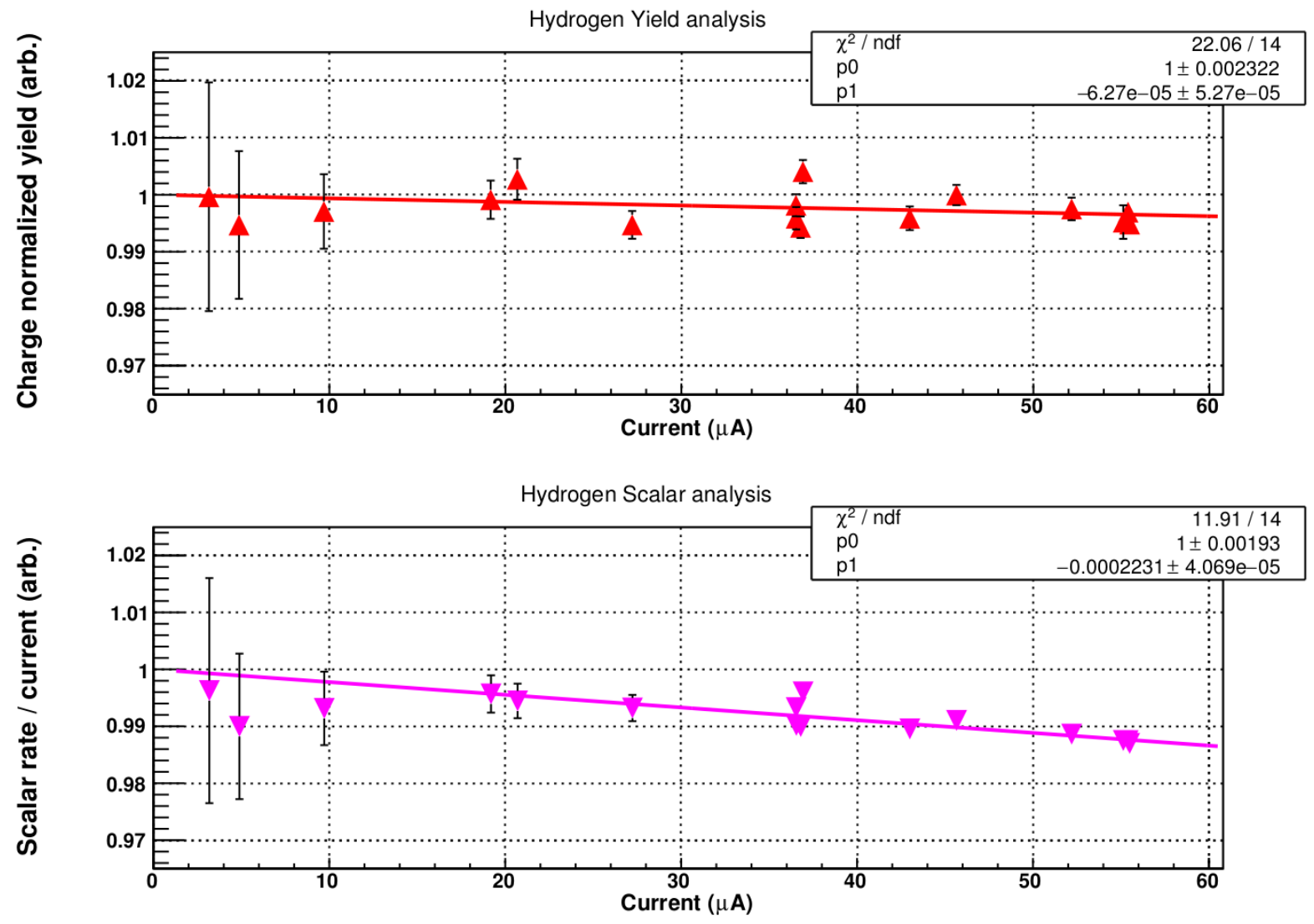

Figure 3.18. Final results of the boiling study on LH2 target showing both yield (top) and scaler analysis (bottom) after correcting for the current offset from B. Schmookler [13]. 


\subsection{Vertical Drift Chamber Tracking Efficiency}

The tracking information of a particle can be obtained from the vertical drift chambers. In order to calculate tracking efficiency an unbiased sample of good electron events is selected. The fraction of this sample which built a track gives the tracking efficiency as discussed in this section. As the VDCs are very efficient, the inefficiency is mainly due to the misreconstruction of particle track by the tracking algorithm. Events can have zero tracks, one track, or multi-tracks. Usually, the number of zero tracks and multi-tracks are very small compared to one track events as shown in Fig. 3.19. When two or more tracks are found to be associated with the same events, multi-track events occur. These events are removed by

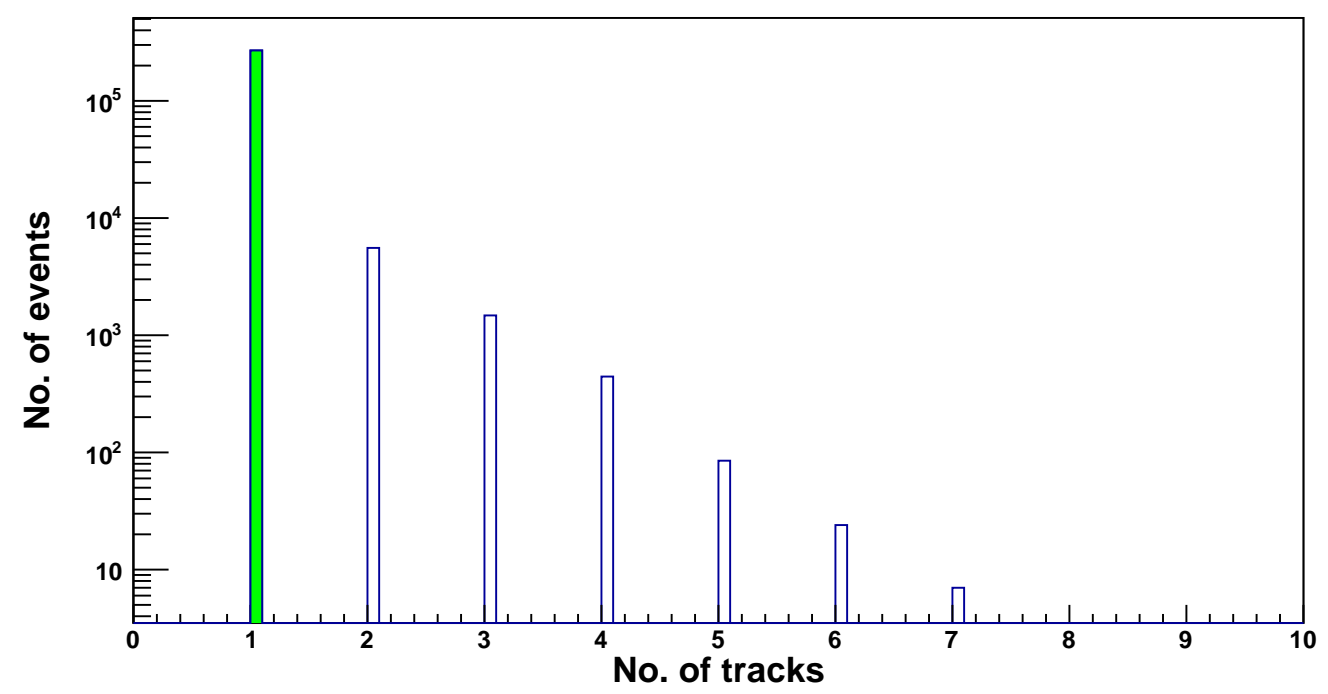

Figure 3.19. Number of tracks reconstructed by the VDC for good electron events and the green distribution represents one track events.

applying a single track cut, which results in an inefficiency for which the yield needs to be corrected. Cuts for the sample selection are:

1. T1 trigger events.

2. Number of Cherenkov photo-electrons greater than 4 . 
3. Normalized total calorimeter energy greater than 0.6.

4. The track beta between 0.4 and 2.0 as shown in Fig. 3.20.

Beta for every event is formed form the scintillator relative times and path length of the scintillator based track. Finally, to get the one track or one cluster efficiency, the fraction of a sample of good electrons with one track in the VDC or one cluster in all four VDC planes is calculated. The table 3.4 shows a comparison of the extracted one track and one

\section{Beta from Scintillators}

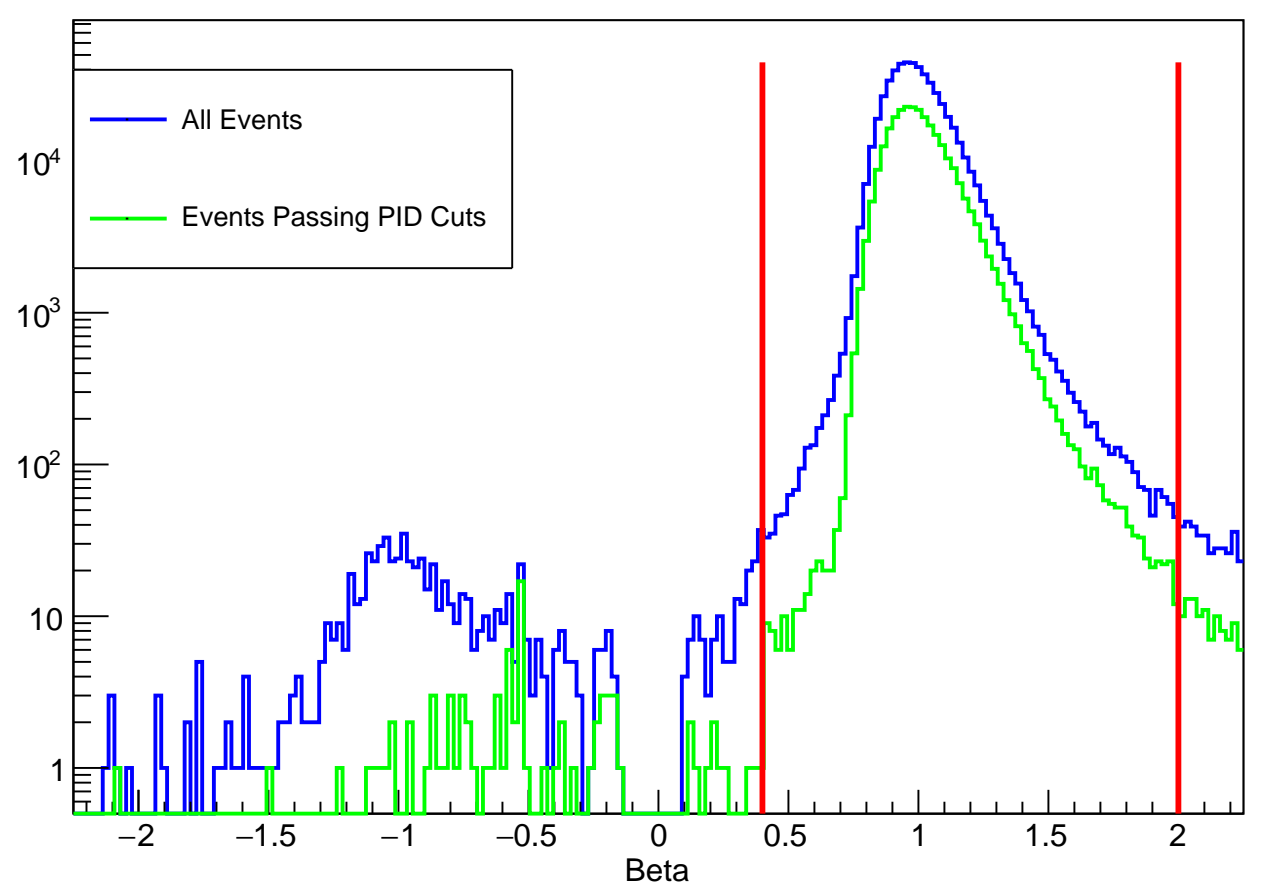

Figure 3.20. The particle beta distribution formed from the scintillator timing information and path-length of the scintillator-based track. The events in the blue distribution shows all trigger one events. The green events the remaining events after the application of the PID cuts. The events between red lines represents good electron events, figure from Ref. [1].

cluster efficiencies for all GMp Fall 2016 kinematics. Here, the statistical uncertainty was calculated assuming binomial distribution. An additional uncertainty of $0.2 \%$ is applied to all extracted VDC efficiencies in order to account the fact that the fraction of one-track or one-cluster events in the selected region may be different from the fraction of multi-track or 
Table 3.4

Comparison table of extracted VDC one-track and one-cluster efficiencies for all GMp Fall 2016 kinematics taken from [1]. One cluster efficiency was used for analysis.

\begin{tabular}{|c|c|c|c|}
\hline Kinematic & Spectrometer & $\epsilon_{1 \text {-track }}$ & $\epsilon_{1-\text { cluster }}$ \\
\hline $\mathrm{k} 1-0.4$ & LHRS & $0.9899\left(9.6 \times 10^{-5}\right)$ & $0.9282\left(2.6 \times 10^{-4}\right)$ \\
\hline $\mathrm{k} 1-1$ & LHRS & $0.9913\left(9.6 \times 10^{-5}\right)$ & $0.9244\left(2.9 \times 10^{-4}\right)$ \\
\hline $\mathrm{k} 1-1.8$ & RHRS & $0.9940\left(9.7 \times 10^{-5}\right)$ & $0.9242\left(3.5 \times 10^{-4}\right)$ \\
\hline $\mathrm{k} 1-1.9$ & RHRS & $0.9940\left(1.3 \times 10^{-4}\right)$ & $0.9244\left(4.8 \times 10^{-4}\right)$ \\
\hline $\mathrm{k} 3-4$ & LHRS & $0.9728\left(6.5 \times 10^{-4}\right)$ & $0.8886\left(1.37 \times 10^{-3}\right)$ \\
\hline $\mathrm{k} 3-6$ & LHRS & $0.9770\left(4.9 \times 10^{-4}\right)$ & $0.9016\left(1.06 \times 10^{-3}\right)$ \\
\hline $\mathrm{k} 3-7$ & LHRS & $0.9800\left(5.1 \times 10^{-4}\right)$ & $0.8799\left(1.27 \times 10^{-3}\right)$ \\
\hline $\mathrm{k} 3-8$ & LHRS & $0.9656\left(8.3 \times 10^{-4}\right)$ & $0.8971\left(1.27 \times 10^{-3}\right)$ \\
\hline $\mathrm{k} 4-9$ & LHRS & $0.9726\left(4.1 \times 10^{-4}\right)$ & $0.8949\left(1.27 \times 10^{-3}\right)$ \\
\hline $\mathrm{k} 4-10$ & LHRS & $0.9744\left(4.0 \times 10^{-4}\right)$ & $0.9015\left(1.27 \times 10^{-3}\right)$ \\
\hline $\mathrm{k} 4-11$ & LHRS & $0.9793\left(6.0 \times 10^{-4}\right)$ & $0.9095\left(1.27 \times 10^{-3}\right)$ \\
\hline $\mathrm{k} 3-9$ & RHRS & $0.9933\left(7.4 \times 10^{-4}\right)$ & $0.9194\left(1.27 \times 10^{-3}\right)$ \\
\hline $\mathrm{k} 4-12$ & RHRS & $0.9846\left(7.6 \times 10^{-4}\right)$ & $0.9198\left(1.27 \times 10^{-3}\right)$ \\
\hline
\end{tabular}

multi-cluster events in the same region. More detail is presented in Ref. [1]. In the study of position dependence performed by B. Schomookler [1] a non-VDC track was reconstructed by using two scintillator s0 and s2, the calorimeter and the straw chamber. The vertical position in s0 was determined by using the time difference of the top and bottom of the scintillator and position in s2 in dispersive direction was determined by using the center of the scintillator and in non-disperisve direction by using the time difference in two PMTs and in calorimeter by using the energy weighted calorimeter block position. The results of VDC one-track and one cluster efficiency studied as a function of $\mathrm{X}$ and $\mathrm{Y}$ position in the focal plane transport coordinate system are shown in Fig.3.21. Clearly, the efficiency shows a significant dependence on Y. However, for this experiment the events of interest are localized within the region indicated by the red line in focal plane as shown in Fig. 3.22, a single number for the VDC efficiency correction is used in analysis. 
VDC 1 Cluster Efficiency vs. 'Track' X

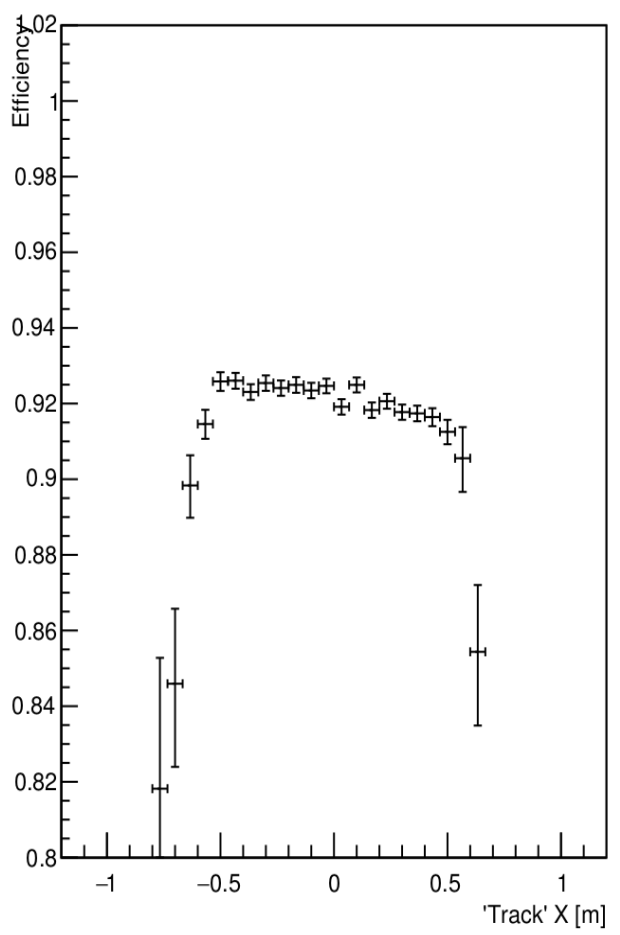

VDC 1 Cluster Efficiency vs. 'Track' Y

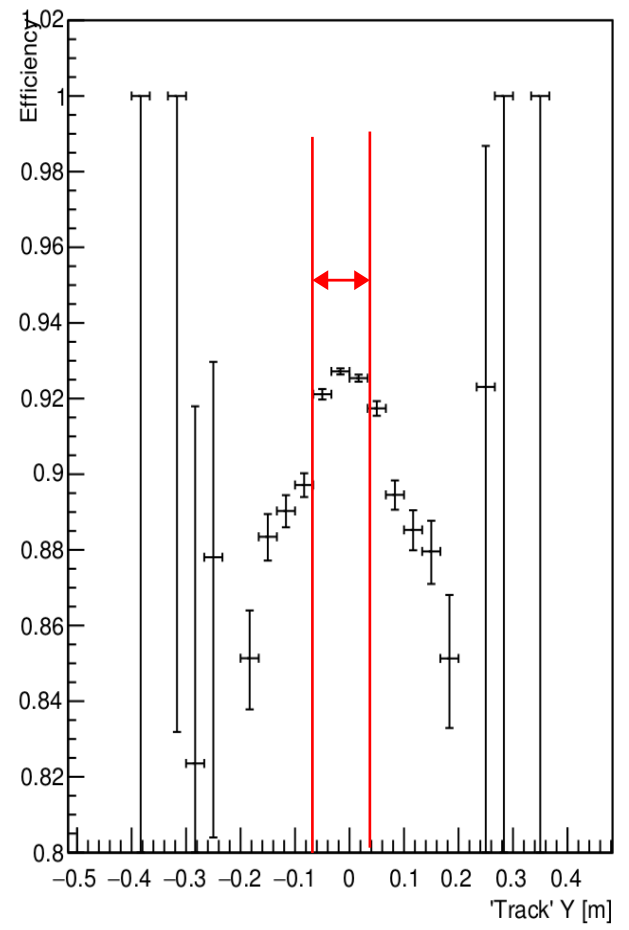

Figure 3.21. Left: VDC single cluster efficiency in $\mathrm{X}$ bins in the focal plane transport coordinate system using the non-VDC based track for an inelastic run on the LHRS. Right: VDC single cluster efficiency in Y bins in the focal plane transport coordinate system. Figure is reproduced from Ref. [1]. 


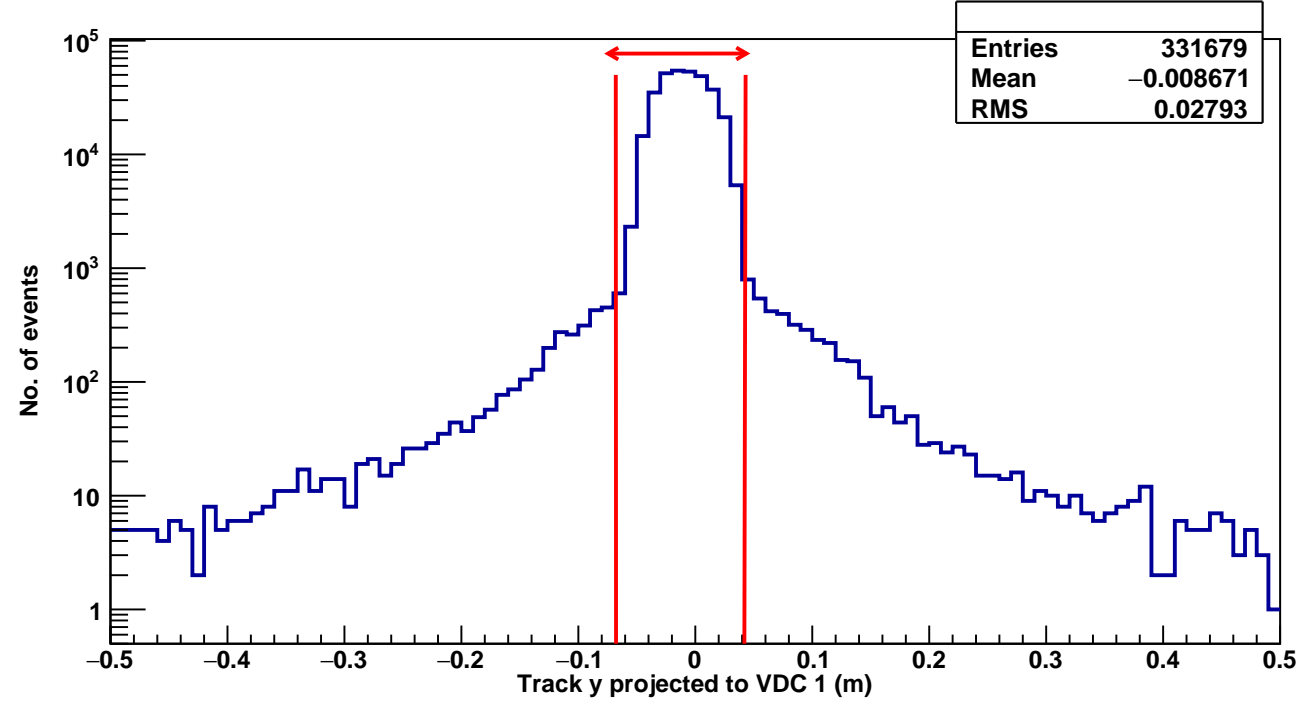

Figure 3.22. Track y in the focal plane transport coordinate system projected to VDC 1 indicating that more than $99 \%$ of events are in the region between the two red lines. 


\subsection{PID Efficiencies}

In all experiments there are the trigger events generated from reactions other than the reaction under study. These events are considered as the background. Subtraction of the background events due to pion initiated triggers is done by using cuts on the gas Cherenkov and calorimeter distributions. The Cherenkov is a threshold detector designed such that an electron will emit Cherenkov radiation while pion will not, whereas the shower calorimeter system provides additional electron rejection capability by detecting the energy deposited by the particle.

Suppose one wants to calculate the efficiency of detector $\mathrm{B}$, call it $\epsilon_{B}$. The idea is to follow the set of particles which have fired both detectors $\mathrm{A}$ and $\mathrm{C}$ and test if they also

fired detector B. Whenever a particle from this set fires detector B, the detector is said to be efficient. In the case a particle fires both detectors $\mathrm{A}$ and $\mathrm{C}$ but does not fire $\mathrm{B}$, then detector B is said to be inefficient. Calling the set of particles which have fired A and C set $\mathrm{X}$ and those from set $\mathrm{X}$ which also fired detector $\mathrm{B}$ set $\mathrm{Y}$, the efficiency of $\mathrm{B}$ can be mathematically expressed as:

$$
\epsilon_{B}=\frac{N_{Y}}{N_{X}},
$$

where $N_{X}$ and $N_{Y}$ represent the number of particles in set $\mathrm{X}$ and set $\mathrm{Y}$, respectively.

\subsubsection{Selection of Events}

Following cuts were applied to calculate the electron cut efficiency and pion rejection efficiency:

\section{Events in $\mathrm{T} 1$}

2. Single cluster cut present in all four VDC planes

3. $\beta 0.3$ to 2.0 to remove cosmic events 
4. Loose "sanity" cuts on the acceptance of

- $-30<y_{\text {tar }}^{\prime}(\operatorname{mrad})<30$

- $-100<x_{t a r}^{\prime}(\operatorname{mrad})<100$

$-3.5 \%<(\%)<5.5$

- $\left|y_{\text {tar }}\right|(\mathrm{m})<0.1 \times \sin (\theta)$

5. The reconstructed invariant mass $(\mathrm{W})$ is between $0.86 \mathrm{GeV} / \mathrm{c}^{2}$ and $1.07 \mathrm{GeV} / \mathrm{c}^{2}$

\subsubsection{Cherenkov Cut Efficiency}

The discrimination of pions from electrons was performed by applying the cuts on the calibrated quantities of Cherenkov and Calorimeter. The cuts can reject unwanted particle such as pions. The majority of the pions that have a signal with more than two photoelectrons are pions that produce delta electrons at the front window or in the gas of the Cherenkov. These delta electrons emit Cherenkov light and the pion is misidentified as an electron. The PID study aims to get the optimized cuts on Cherenkov and the calorimeter which can hereby eliminate pions while keeping as many electrons as possible. In order to get the electron cut efficiency of the Cherenkov, the electron events are selected in pion rejector as shown in red box on the bottom left panel of Fig. 3.23 and then one counts those events that also fired Cherenkov above some cut as shown in bottom right panel of the Fig. 3.23.

If the pion background in an experiment is large, evaluation of the percentage of remaining pion mixed into the electron events is also important. In order to get the pion rejection efficiency of the Cherenkov, the pion events are selected in pion rejector as shown in the blue box on bottom left panel of Fig. 3.23, and then one counts those events that also fired Cherenkov below some cut shown in bottom right panel of Fig. 3.23. The cuts used for these study are VDC one cluster cuts and loose acceptance cuts. The electron cut efficiency is 

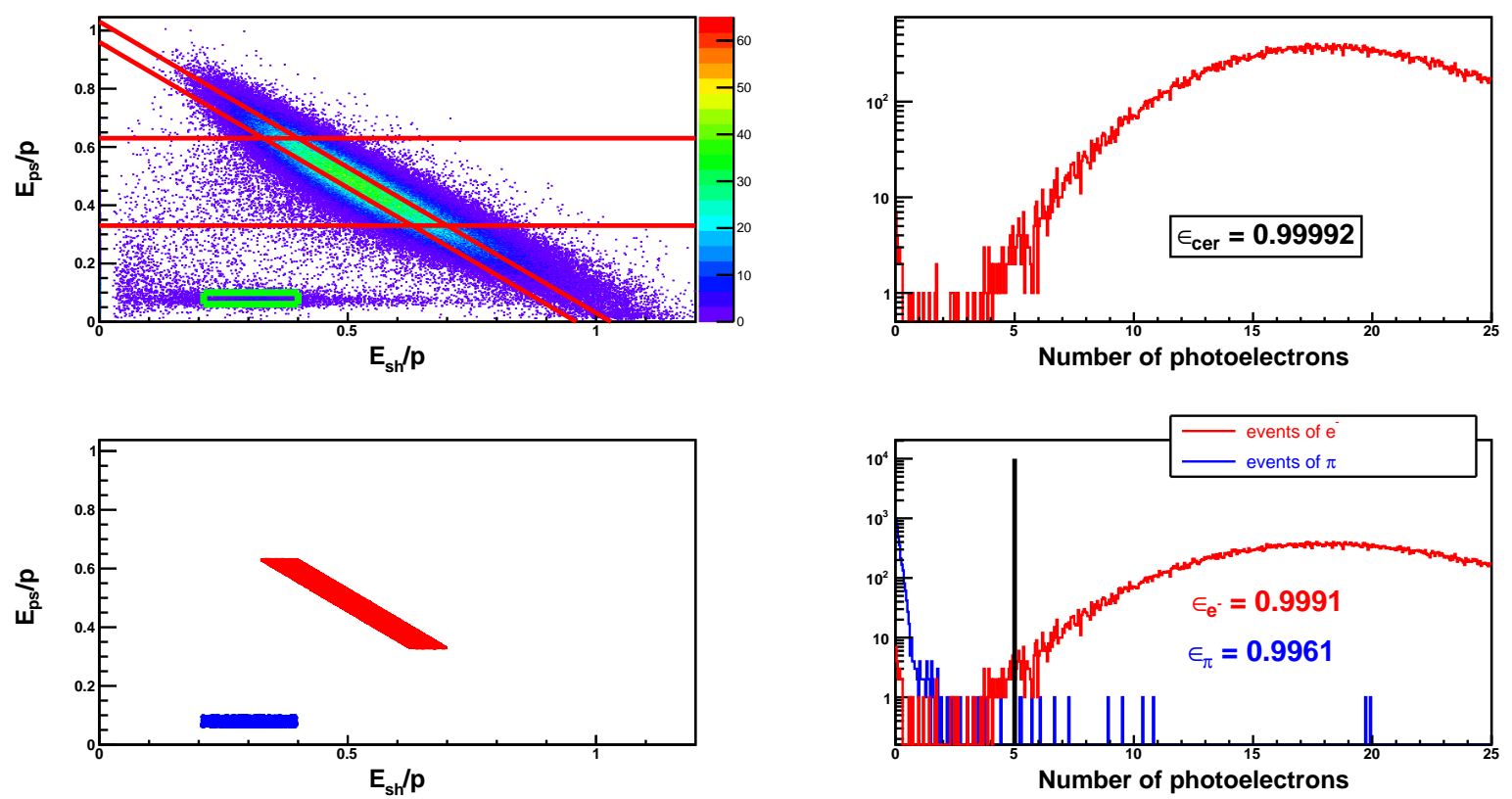

Figure 3.23. Top and bottom left panels shows the electron and pion sample selected in calorimeter to study the gas Cherenkov efficiency, where the events inside the red lines are the electrons and the events inside blue rectangle are the pions. The top left panel shows the number of photo-electrons collected in the gas Cherenkov for the electron(red) selected in top left panel. The last panel plot shows the Cherenkov cut efficiency for the electron (red) of $99.91 \%$ and pion rejection percentage (blue) of $99.96 \%$ selected in top left plot (These are the preliminary results for one of the low $Q^{2}$ Spring 2016 RHRS kinematics presented to outline the procedure. The final results for all Fall 2016 kinematics were performed by B. Schmookler [1] based on this procedure).

given by

$$
\epsilon_{c e r}^{e^{-}}=\frac{N_{c e r}^{e^{-}}}{N_{c a l}^{e^{-}}}
$$

Also, the pion rejection efficiency is defined as the fraction of pions removed by applying PID cuts:

$$
\epsilon_{c e r}^{\pi^{-}}=\frac{N_{c e r}^{\pi^{-}}}{N_{c a l}^{\pi^{-}}}
$$

Here, $N_{c a l}^{e^{-}}\left(N_{c a l}^{\pi^{-}}\right)$is the electron (pion) samples from calorimeter whereas $N_{c e r}^{e^{-}}$is the number of electrons left over after cutting on the Cherenkov and $N_{c e r}^{\pi^{-}}$is the number of pions rejected. In Fig. 3.23 the $\mathrm{x}$-axis and $\mathrm{y}$-axis are total energy deposited by the first layer (pre-shower) 


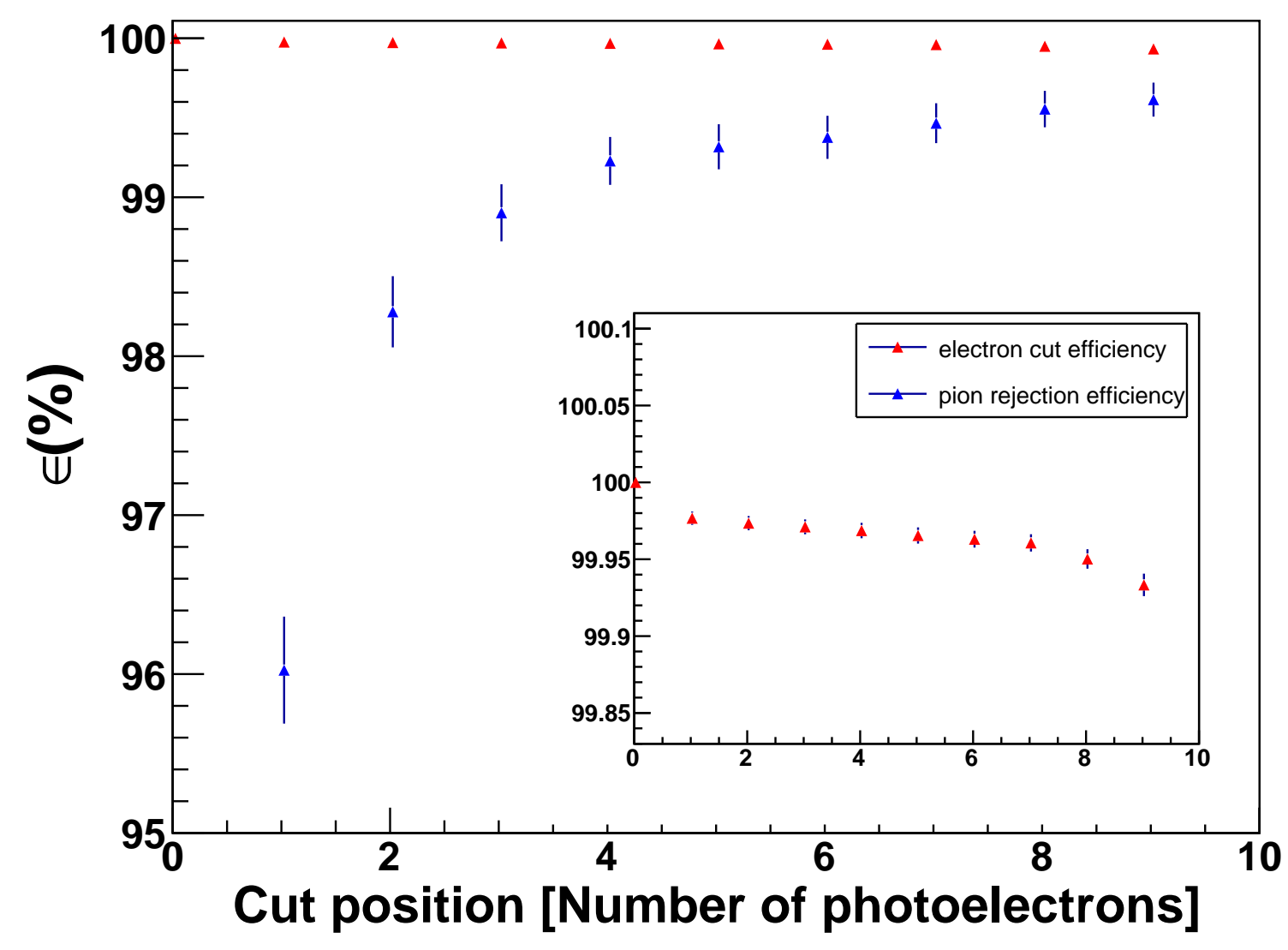

Figure 3.24. Electron and pion cherenkov cut efficiencies as a function of ADC sum.

and the second layer (shower) of the calorimeter showing the cuts used to select electron samples(red) and pion samples(blue) shows the number of electrons left over after the cut in Cherenkov, where red distribution are the electrons and blue distributions are pions. Figure 3.24 shows cut efficiency as a function of Cherenkov ADC sum. The cut efficiency of electrons is indicated by red distributions and that of pions are blue distributions. The uncertainty in efficiency is calculated by assuming standard binomial distribution:

$$
\sigma(\epsilon)=\sqrt{\frac{\epsilon(1-\epsilon)}{N_{c a l}}},
$$




\subsubsection{Calorimeter Efficiency}

A calorimeter cut to reduce the number of pions that pass the Cherenkov cut was applied. The PID can be improved by applying a cut on the first layer of calorimeter and a cut on
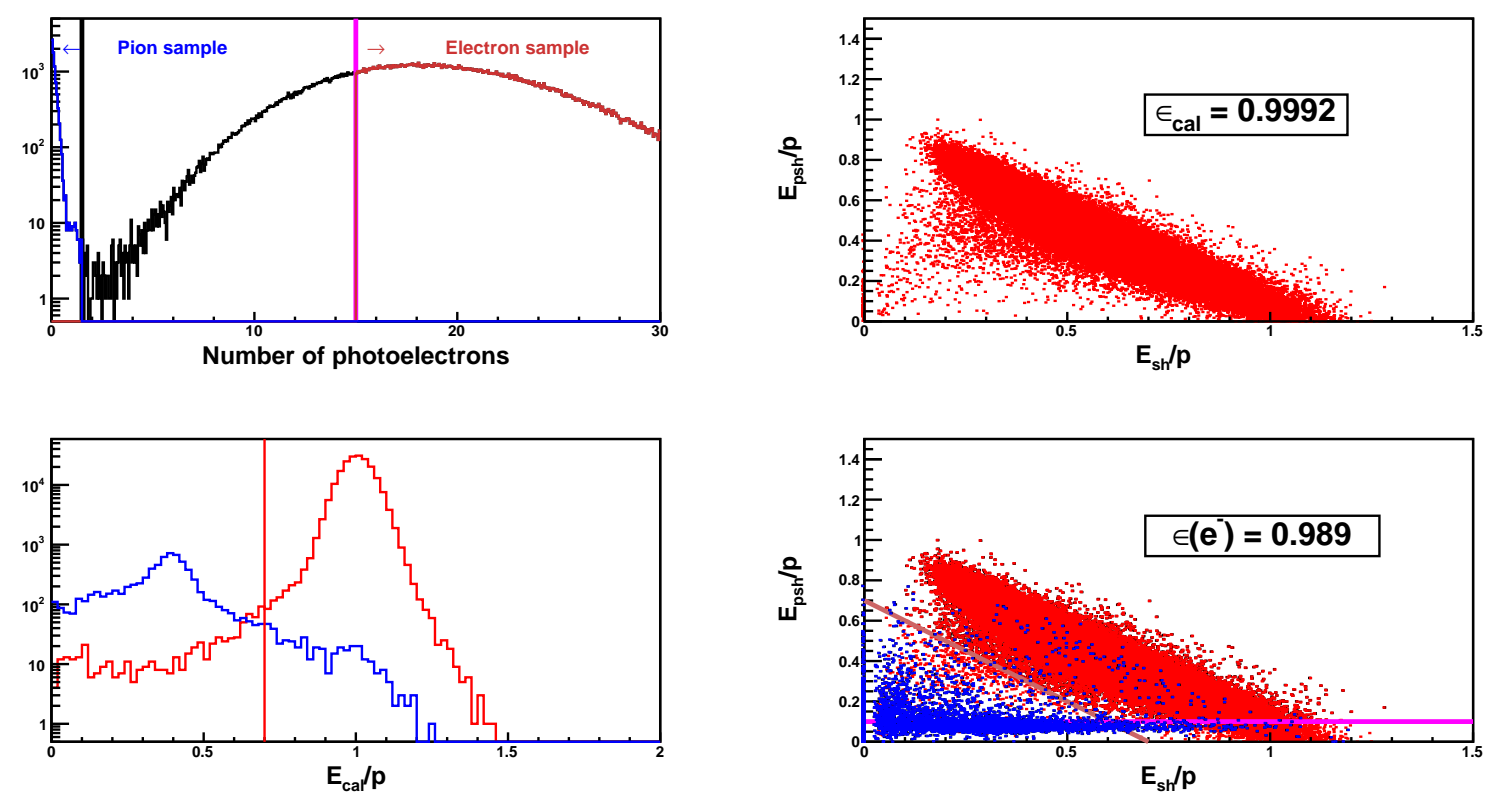

Figure 3.25. Event samples selected in Cherenkov to determine calorimeter detection and cut efficiencies

the total energy deposited in calorimeter per unit particle momentum. The Cherenkov and anti-Cherenkov cuts were applied to determine electron(red) and pion(blue) as shown in bottom left plot of Fig. 3.25 . Then the calorimeter cut efficiency is given by,

$$
\epsilon_{c a l}^{e^{-}}=\frac{N_{c a l}^{e^{-}}}{N_{c e r}^{e^{-}}}
$$

Also, the pion rejection efficiency is defined as the fraction of pions removed by applying PID cuts:

$$
\epsilon_{c a l}^{\pi^{-}}=\frac{N_{c a l}^{\pi^{-}}}{N_{c e r}^{\pi^{-}}}
$$




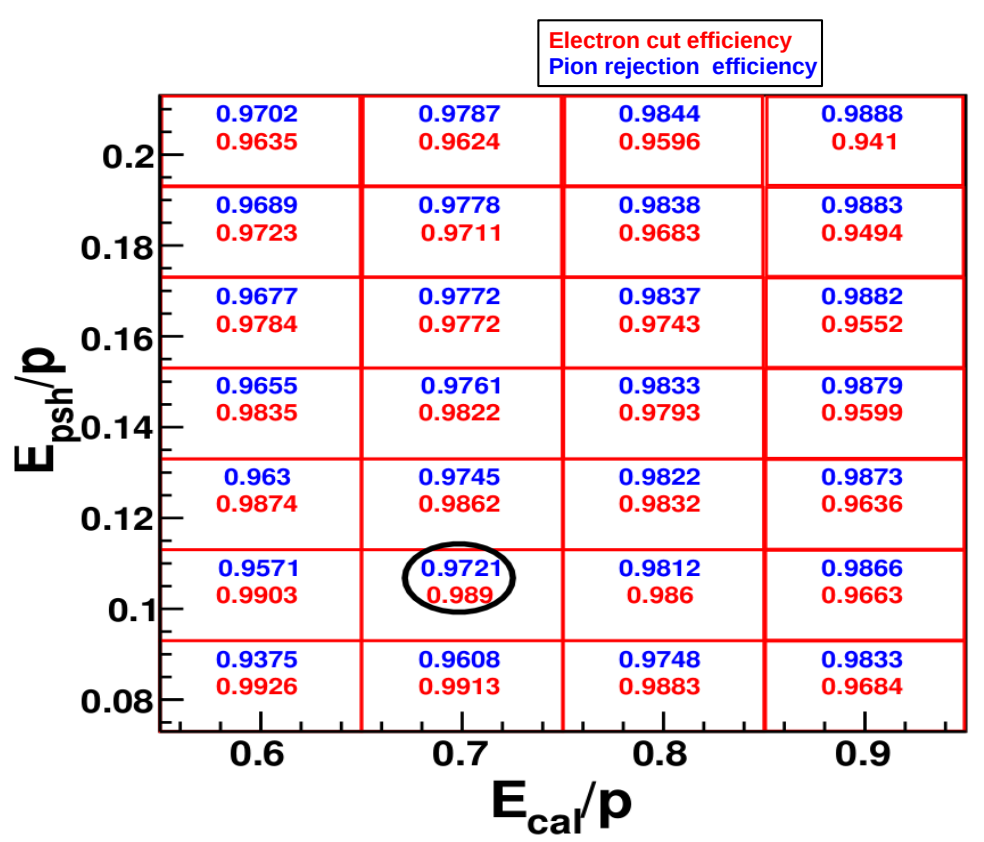

Figure 3.26. Grid showing electron cut efficiencies(red) and pion rejection efficiencies(blue) on the total energy deposited in the calorimeter and the energy deposited in layer 1

Here, $N_{c e r}^{e^{-}}\left(N_{c e r}^{\pi^{-}}\right)$is the electron (pion) samples from Cherenkov whereas $N_{c a l}^{e^{-}}$is the number of electrons left over after cutting on the calorimeter and $N_{c e r}^{\pi^{-}}$is the number of pions rejected. The bottom right plot shows the electron(red) and pions (blue) events selected in Cherenkov that also triggered calorimeter. And the cut efficiency obtained for this kinematics was about $98.9 \%$.

In order to find the best cut to select good electron events in the calorimeter the efficiencies are calculated in different bins in pre-shower vs $E_{c a l} / p$, which is shown in Fig. 3.26. For the kinematics chosen the best cuts which gives good electron cut efficiency and good pion rejection efficiency are $E_{p s} / p>0.1$ and $E_{c a l} / p>0.7$. To determine the total number of good electrons, $N_{e^{-}}^{t}, N^{t}=N_{e^{-}}^{t}+N_{\pi}^{t}$ was measured before PID cuts and $N^{f}=N_{e^{-}}^{f}+N_{\pi}^{f}$ after PID cut. Using the electron cut efficiency for the gas Cherenkov (calorimeter) by selecting a sample of clean electrons in the calorimeter (gas Cherenkov), and then looking at the response of the gas Cherenkov (calorimeter) for that sample one can calculate the total 
electron efficiency $\epsilon_{e^{-}}$and the total pion rejection efficiency $\left(\epsilon_{\pi}\right)$ as $N_{e^{-}}^{f}=\epsilon_{e^{-}} \times N_{e^{-}}^{t}$ and $N_{\pi}^{f}=\left(1-\epsilon_{\pi}\right) \times N_{\pi}^{t}$. Since

$$
\begin{aligned}
N^{f} & =N_{e^{-}}^{f}+N_{\pi}^{f} \\
& =N_{e^{-}}^{f} \cdot\left(1+\frac{N_{\pi}^{f}}{N_{e^{-}}^{f}}\right)
\end{aligned}
$$

The total number of good electrons in terms of the final number of pion-to-electron ratio $\left(\frac{\pi}{e^{-}}\right)_{f}$ can be written as

$$
N_{e^{-}}^{t}=\frac{N_{e^{-}}^{f}}{\epsilon_{e-} \cdot\left(1+\left(\frac{\pi}{e^{-}}\right)_{f}\right)}
$$

In order to correct the PID efficiency for each run, $\epsilon_{e^{-}}$and $\left(\frac{\pi}{e^{-}}\right)_{f}$ were determined. The

Table 3.5

The table of PID cut efficiencies for all Fall 2016 GMp kinematics. Table is from Ref. [1].

\begin{tabular}{|c|c|c|c|c|c|}
\hline $\begin{array}{c}\text { Kinematic } \\
\mathrm{k} 1-0.4\end{array}$ & $\begin{array}{c}\text { Spectrometer } \\
\text { LHRS }\end{array}$ & $\begin{array}{c}\epsilon_{e^{-}} \\
0.995\end{array}$ & $\begin{array}{c}\epsilon_{\pi} \\
0.977\end{array}$ & $\begin{array}{c}\left(\frac{\pi}{e^{-}}\right)_{i} \\
7.4 \times 10^{-4}\end{array}$ & $\begin{array}{c}\left(\frac{\pi}{e^{-}}\right)_{f} \\
1.7 \times 10^{-5}\end{array}$ \\
\hline $\mathrm{k} 1-1$ & LHRS & 0.995 & 0.999 & $9.3 \times 10^{-4}$ & $5.7 \times 10^{-6}$ \\
\hline $\mathrm{k} 1-1.8$ & RHRS & 0.992 & 0.999 & 0.025 & $2.9 \times 10^{-6}$ \\
\hline $\mathrm{k} 1-1.9$ & RHRS & 0.991 & 0.999 & 0.020 & $2.8 \times 10^{-6}$ \\
\hline $\mathrm{k} 3-4$ & LHRS & 0.992 & 0.999 & $8.7 \times 10^{-3}$ & $2.8 \times 10^{-6}$ \\
\hline $\mathrm{k} 3-6$ & LHRS & 0.993 & 0.999 & 0.057 & $2.3 \times 10^{-5}$ \\
\hline $\mathrm{k} 3-7$ & LHRS & 0.993 & 0.999 & 0.25 & $4.0 \times 10^{-5}$ \\
\hline $\mathrm{k} 3-8$ & LHRS & 0.994 & 0.999 & 1.07 & $1.8 \times 10^{-4}$ \\
\hline $\mathrm{k} 4-9$ & LHRS & 0.991 & 0.999 & 0.20 & $7.6 \times 10^{-5}$ \\
\hline $\mathrm{k} 4-10$ & LHRS & 0.992 & 0.999 & 0.55 & $2.3 \times 10^{-4}$ \\
\hline $\mathrm{k} 4-11$ & LHRS & 0.992 & 0.999 & 2.91 & $6.6 \times 10^{-4}$ \\
\hline $\mathrm{k} 3-9$ & RHRS & 0.984 & 0.999 & 7.28 & $4.2 \times 10^{-4}$ \\
\hline $\mathrm{k} 4-12$ & RHRS & 0.984 & 0.999 & 10.42 & $8.4 \times 10^{-4}$ \\
\hline
\end{tabular}

total electron efficiency is calculated from the individual Cherenkov and calorimeter electron efficiencies as $\epsilon_{e^{-}}=\epsilon_{c e r, e^{-}} \times \epsilon_{c a l, e^{-}}$. The total pion rejection efficiency is calculated from the 
individual Cherenkov and calorimeter pion rejection efficiencies as $\epsilon_{\pi}=1-\left(1-\epsilon_{c e r, \pi}\right) \times(1-$ $\left.\epsilon_{c a l, \pi}\right)$. Table 3.5 shows the final total electron detection efficiencies, the total pion rejection efficiencies, the initial pion-to-electron ratios, and the final pion-to-electron ratios for the following set of PID cuts (for all Fall 2016 kinematics) :

$$
N_{p e}>4,\left(\frac{E}{P}\right)_{\text {Total }}>0.7,\left(\frac{E}{P}\right)_{\text {Layer } 1}>0.08
$$

For each measured efficiency, a standard binomial statistical uncertainty was calculated. It

\section{Table 3.6}

The relative uncertainties on electron efficiency, pion rejection efficiency, and final pion-toelectron ratio for all Fall 2016 GMp kinematics. An uncertainty of $5 \times 10^{-4}\left(1 \times 10^{-4}\right)$ on the electron (pion) efficiency due to the sample selection and also applied an uncertainty of $1 \times 10^{-4}$ on both the electron and pion efficiency due to the E/p threshold. Table is from Ref. [1]

\begin{tabular}{|c|c|c|c|c|}
\hline $\begin{array}{c}\text { Kinematic } \\
\text { k1-0.4 }\end{array}$ & $\begin{array}{c}\text { Spectrometer } \\
\text { LHRS }\end{array}$ & $\begin{array}{c}d \epsilon_{e} / \epsilon_{e} \\
5.2 \times 10^{-4}\end{array}$ & $\begin{array}{c}d \epsilon_{\pi} / \epsilon_{\pi} \\
4.5 \times 10^{-2}\end{array}$ & $\begin{array}{c}d\left(\frac{\pi}{e}\right)_{f} /\left(\frac{\pi}{e}\right)_{f} \\
3.6 \times 10^{-2}\end{array}$ \\
\hline $\mathrm{k} 1-1$ & LHRS & $5.2 \times 10^{-4}$ & $3.2 \times 10^{-3}$ & $1.1 \times 10^{-2}$ \\
\hline $\mathrm{k} 1-1.8$ & RHRS & $5.3 \times 10^{-4}$ & $1.7 \times 10^{-3}$ & $8.2 \times 10^{-3}$ \\
\hline $\mathrm{k} 1-1.9$ & RHRS & $5.4 \times 10^{-4}$ & $1.2 \times 10^{-3}$ & $1.2 \times 10^{-2}$ \\
\hline $\mathrm{k} 3-4$ & LHRS & $9.3 \times 10^{-4}$ & $1.3 \times 10^{-2}$ & $6.3 \times 10^{-2}$ \\
\hline $\mathrm{k} 3-6$ & LHRS & $7.4 \times 10^{-4}$ & $5.2 \times 10^{-3}$ & $2.1 \times 10^{-2}$ \\
\hline $\mathrm{k} 3-7$ & LHRS & $7.6 \times 10^{-4}$ & $2.1 \times 10^{-3}$ & $1.1 \times 10^{-2}$ \\
\hline $\mathrm{k} 3-8$ & LHRS & $8.4 \times 10^{-4}$ & $1.2 \times 10^{-3}$ & $8.7 \times 10^{-3}$ \\
\hline $\mathrm{k} 4-9$ & LHRS & $8.8 \times 10^{-4}$ & $3.3 \times 10^{-3}$ & $1.3 \times 10^{-2}$ \\
\hline $\mathrm{k} 4-10$ & LHRS & $8.1 \times 10^{-4}$ & $2.2 \times 10^{-3}$ & $8.4 \times 10^{-3}$ \\
\hline $\mathrm{k} 4-11$ & LHRS & $1.1 \times 10^{-3}$ & $1.4 \times 10^{-3}$ & $8.8 \times 10^{-3}$ \\
\hline $\mathrm{k} 3-9$ & RHRS & $2.0 \times 10^{-3}$ & $7.8 \times 10^{-4}$ & $1.4 \times 10^{-2}$ \\
\hline $\mathrm{k} 4-12$ & RHRS & $1.5 \times 10^{-3}$ & $5.3 \times 10^{-4}$ & $9.9 \times 10^{-3}$ \\
\hline
\end{tabular}

is clear from the table 3.5 that the final pion-to-electron ratios are very small which were determined by the cut efficiencies and by the initial pion-to-electron ratio. The relative uncertainty on the final pion-to-electron ratio is also shown in table 3.6 for each kinematic. 


\subsection{Computer and Electronic Dead Time}

During data taking some events get lost because the DAQ computer is busy processing an event and not available to process new events. The computer live time is measured by taking the ratio of the number of events recorded $\left(N_{R e c}\right)$ from the main trigger to the number of counts in the main trigger scaler $\left(N_{\text {Total }}\right)$. i.e.

$$
\text { Computer Live time }=\frac{N_{\text {Rec }}}{N_{\text {Total }}}
$$

To correct for this live time, the measured yield is divided by the computer live time. The table 3.7 shows the computer live time for all GMp Fall 2016 kinematics, along with relative uncertainties calculated assuming binomial errors.

Table 3.7

Computer live-times for all GMp Fall 2016 kinematics. The relative uncertainty were calculated assuming binomial errors. Table is taken from Ref. [1].

\begin{tabular}{|c|c|c|}
\hline Kinematic & Spectrometer & Computer Live-Time \\
\hline $\mathrm{k} 1-0.4$ & LHRS & $0.6935\left(5.1 \times 10^{-4}\right)$ \\
\hline $\mathrm{k} 1-1$ & LHRS & $0.9454\left(2.2 \times 10^{-4}\right)$ \\
\hline $\mathrm{k} 1-1.8$ & RHRS & $0.9171\left(3.3 \times 10^{-4}\right)$ \\
\hline $\mathrm{k} 1-1.9$ & RHRS & $0.9207\left(5.1 \times 10^{-4}\right)$ \\
\hline $\mathrm{k} 3-4$ & LHRS & $0.9654\left(4.2 \times 10^{-4}\right)$ \\
\hline $\mathrm{k} 3-6$ & LHRS & $0.9774\left(3.1 \times 10^{-4}\right)$ \\
\hline $\mathrm{k} 3-7$ & LHRS & $0.9794\left(1.1 \times 10^{-4}\right)$ \\
\hline $\mathrm{k} 3-8$ & LHRS & $0.9843\left(3.0 \times 10^{-4}\right)$ \\
\hline $\mathrm{k} 4-9$ & LHRS & $0.9742\left(2.5 \times 10^{-4}\right)$ \\
\hline $\mathrm{k} 4-10$ & LHRS & $0.9728\left(2.6 \times 10^{-4}\right)$ \\
\hline $\mathrm{k} 4-11$ & LHRS & $0.9790\left(3.6 \times 10^{-4}\right)$ \\
\hline $\mathrm{k} 3-9$ & RHRS & $0.9684\left(5.4 \times 10^{-4}\right)$ \\
\hline $\mathrm{k} 4-12$ & RHRS & $0.9547\left(6.5 \times 10^{-4}\right)$ \\
\hline
\end{tabular}

Electronic dead time arises when events are not recorded by the scaler counter. The fraction of lost events per recorded event is given by

$$
\text { Number lost }=\text { Raw rate } \times \tau
$$


where $\tau$ is the electronic dead time. In order to correct for electronic dead time the measured yield is multiplied by the following factor:

$$
\text { Correction factor }=1+\text { Raw rate } \times \tau
$$

A correction factor was calculated by multiple coincidence units with different output widths. The correction due to the electronic dead time is at most $2 \times 10^{-4}$.

\subsection{HRS Optics}

The GMp experiment used four different spectrometer configurations due to the replacement of the first quadruple (Q1) on both LHRS and RHRS. In Fall 2015 the quadrupole from the short orbit spectrometer (SOS) in Hall C was installed in the RHRS to replace the failing Q1. Later in 2016 the LHRS Q1 became non-functional, so it was replaced by the SOS quad from RHRS and a new resistive quad was installed in RHRS. Reconstruction of the invariant mass in electron-proton elastic scattering requires the precise determination of position and angle of the electron at the vertex. The optical transport of the HRS describes the transportation of charged particles from the target to the focal plane, while the optics reconstruction describes the reconstruction of the momentum and vertex of the scattered particle at the target. That is,

$$
W_{t a r}=[\text { optics matrix }] W_{f p},
$$

where, $W_{t a r}$ are the target quantities and $W_{f p}$ are the focal plane quantities. The reconstruction matrix elements are determined through an established optics calibration procedure. Data taken with sieve slit and a multi-foil target were used for angle and position optimization whereas, momentum calibration was performed by scanning the central momentum of the spectrometer around the elastic peak. In order to describe the optical properties of the 
spectrometer, the Cartesian coordinate systems have been defined, which are discussed in section 2.9 .

\subsubsection{Optics Optimization Procedure}

The focal plane coordinates measured by the pair of VDCs are used to calculate target

coordinates. In this procedure $x_{t g}$ is fixed at zero during the optimization, such that the target quantities are reduced to the four quantities: $\delta, x_{t g}^{\prime}, y_{t g}$ and $y_{t g}^{\prime}$. This gives the relationship between focal plane and target coordinates in first order approximation:

$$
\left[\begin{array}{c}
\delta \\
x_{t a r}^{\prime} \\
y_{t a r} \\
y_{t a r}^{\prime}
\end{array}\right]_{t g}=\left[\begin{array}{cccc}
<\delta \mid x> & <\delta \mid x_{t a r}^{\prime}> & 0 & 0 \\
<x_{t a r}^{\prime} \mid x> & <x_{t a r}^{\prime} \mid x_{t a r}^{\prime}> & 0 & 0 \\
0 & 0 & <y \mid y> & <y \mid y_{t a r}^{\prime}> \\
0 & 0 & <y_{t a r}^{\prime} \mid y> & <y_{t a r}^{\prime} \mid y_{t a r}^{\prime}>
\end{array}\right]\left[\begin{array}{c}
x \\
x^{\prime} \\
y_{t a r}^{\prime} \\
y
\end{array}\right]_{f p}
$$

The zero elements result from the mid plane symmetry of the spectrometer. The focal plane coordinates and the target coordinates are linked by a set of tensors $D_{j k l}, T_{j k l}, Y_{j k l}$ and $P_{j k l}$ according to $[48]$

$$
\begin{aligned}
\delta & =\sum_{j k l} D_{j k l} x_{f p}^{i} y_{f p}^{k} y_{f p}^{\prime l} \\
x_{t g}^{\prime} & =\sum_{j k l} T_{j k l} x_{f p}^{\prime i} y_{f p}^{k} y_{f p}^{\prime l} \\
y_{t g} & =\sum_{j k l} Y_{j k l} x_{f p}^{i} y_{f p}^{k} y_{f p}^{\prime l} \\
y_{t g}^{\prime} & =\sum_{j k l} P_{j k l} x_{f p}^{\prime i} y_{f p}^{k} y_{f p}^{\prime l},
\end{aligned}
$$


where the subscripts denote the power of each focal plane variable and the transformation tensors $D_{j k l}, T_{j k l}, Y_{j k l}$ and $P_{j k l}$ are polynomials in $x_{f p}$. Where,

$$
P_{j k l}=\sum_{i=0}^{m} C_{i} x_{f p}^{i}
$$

The final transformation for $y_{t g}^{\prime}$ for example is given by:

$$
y_{t g}^{\prime}=\sum_{j k l} \sum_{i=1}^{m} C_{i} x_{f p}^{i} x_{f p}^{\prime j} y_{f p}^{k} y_{f p}^{\prime l}
$$

To optimize the transport tensors we performed a $\chi^{2}$ minimization on the difference between the reconstructed target variables and the expected values. To calibrate $x_{t a r}^{\prime}, y_{\text {tar }}^{\prime}$ and $y_{t a r}$, a fixed energy electron beam with a point-like profile is incident on a multi-foil target. The foils are aligned along the beam line so that HCS coordinates $x_{\text {beam }}$ and $y_{\text {beam }}$ of the interaction position are determined by the BPMs. The vertical coordinate $x_{t a r}$ is determined by the beam position. $y_{\text {tar }}$ and $z_{\text {react }}$ are the horizontal track position in the TCS and the vertex position along the ideal beam direction, respectively. In addition to the foil, a sieve slit collimator is placed before the entrance of the spectrometer.
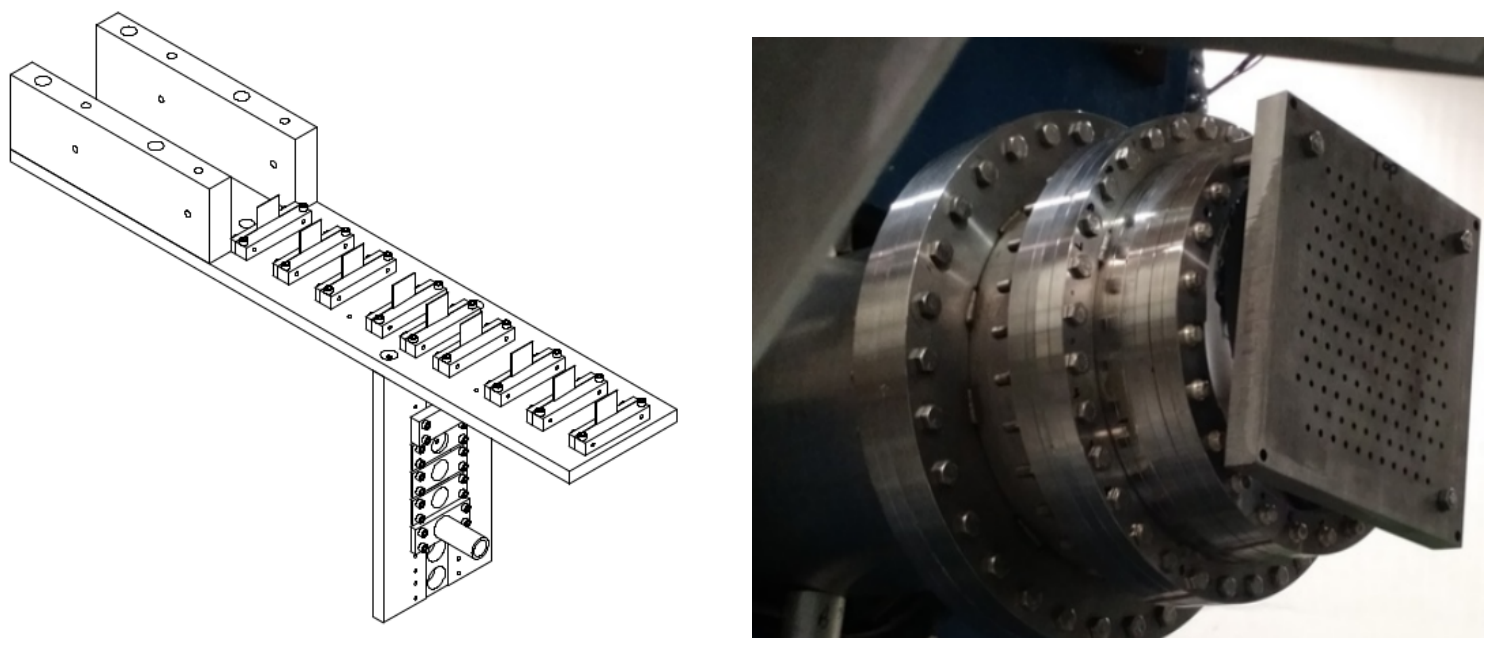

Figure 3.27. Schematic of multi-foil carbon target and Sieve collimator [14] used for Fall 2106 optics optimization. 
Fig. 3.27 shows the multi-foil carbon target and sieve slit on the left HRS used in this experiment for the 2016 run. The DIS electrons were used for vertex and angle calibration. The sieve has holes that are arranged in a grid pattern with well-defined horizontal and vertical coordinates at the sieve plane $x_{\text {sieve }}$ and $y_{\text {sieve }}$ in the TCS. The following are the expressions for those sieve plane variables:

$$
\begin{aligned}
& x_{\text {sieve }}=x_{t a r}+L x_{t a r}^{\prime} \\
& y_{\text {sieve }}=y_{\text {tar }}+L y_{\text {tar }}^{\prime} \\
& z_{\text {react }}=-(y+D) \frac{\cos \left(\tan ^{-1} y_{\text {tar }}^{\prime}\right)}{\sin \left(\theta_{0}+\tan ^{-1} y_{\text {tar }}^{\prime}\right)}+x_{\text {beam }} \cot \left(\theta_{0}+\tan ^{-1} y_{\text {tar }}^{\prime}\right),
\end{aligned}
$$

where,

$$
\begin{aligned}
x_{\text {tar }}^{\prime} & =\frac{x_{\text {sieve }}+D_{x}+y_{\text {beam }}}{L-z_{\text {react }} \cos \theta_{0}-x_{\text {beam }} \sin \theta_{0}}, \\
y_{\text {tar }}^{\prime} & =\frac{y_{\text {sieve }}+D_{y}-x_{\text {beam }} \cos \theta_{0}+z_{\text {react }} \sin \theta_{0}}{L-z_{\text {react }} \cos \theta_{0}-x_{\text {beam }} \sin \theta_{0}}
\end{aligned}
$$

Where, $\theta_{0}$ is the spectrometer central angle, $L$ is the distance from the sieve slit to the TCS origin and $D_{x}$ and $D_{y}$ are the vertical and horizontal deviations of the spectrometer central ray from the HCS determined from survey, respectively. The optics matrix elements are optimized by minimizing the following function:

$$
\chi^{2}=\sum_{i=0}^{\text {Events }}\left(X_{\text {sieve }}^{i}-X_{\text {sieve }}^{0}\right)^{2}
$$

where, $X_{\text {sieve }}$ is $x_{\text {sieve }}$ or $y_{\text {sieve }}$ and $X_{\text {sieve }}^{0}$ are corresponds to the surveyed location of the sieve hole.

The momentum calibration is performed by scanning the central momentum of the spectrometer, $p_{0}$, around the elastic peak, i.e. $\delta$ scans at $p_{0}=p_{\text {elastic }}, \pm 2 \%$ and $\pm 4 \%$. The true momentum is calculated using the scattering angle after accounting for energy loss in the 
target material. The energy loss is calculated using the most probable energy loss described by the highly-skewed Landau-Vavilov distribution, given the material and path length as discussed later in the Section 3.12. The true momentum of the detected electron is then determined from:

$$
E^{\prime}=P(M, \theta)=\frac{E}{1+\frac{E}{M}(1-\cos \theta)},
$$

where $\mathrm{M}$ is the target mass, $\mathrm{E}$ is the beam energy, and $\theta$ is the scattering angle. The deviation of the particle momentum from the central momentum of the spectrometer $\left(p_{0}\right)$ is given by:

$$
\delta=\frac{p-p_{0}}{p_{0}}
$$

The optimization is performed by minimizing the $\chi^{2}$ utilizing Minuit2 [49] based routine and

$$
\chi^{2}=\sum_{i=0}^{\text {Events }}\left(\delta^{\text {cal }}-\delta^{0}\right)^{2}
$$

where, $\delta^{0}$ corresponds to the reference variable calculated from the elastic condition.

\subsubsection{Target y Calibration}

In the hall coordinate system, $y_{\text {tar }}$ is related to the foil positions along $z$-direction. To optimize, $y_{\text {tar }}$ reconstructed foil positions were matched with the real foil positions. The Spring 2016 run used five foils, whereas, the Fall 2016 run used nine foils to provide additional $y_{\text {tar }}$ constraints. Fig. 3.28 represents the calibration results of $y_{\text {tar }}$ for the Spring 2016 run period. The blue lines indicates the true positions. Clearly, Gaussian fitted mean value of the reconstructed foil target position are close to the real target positions. 

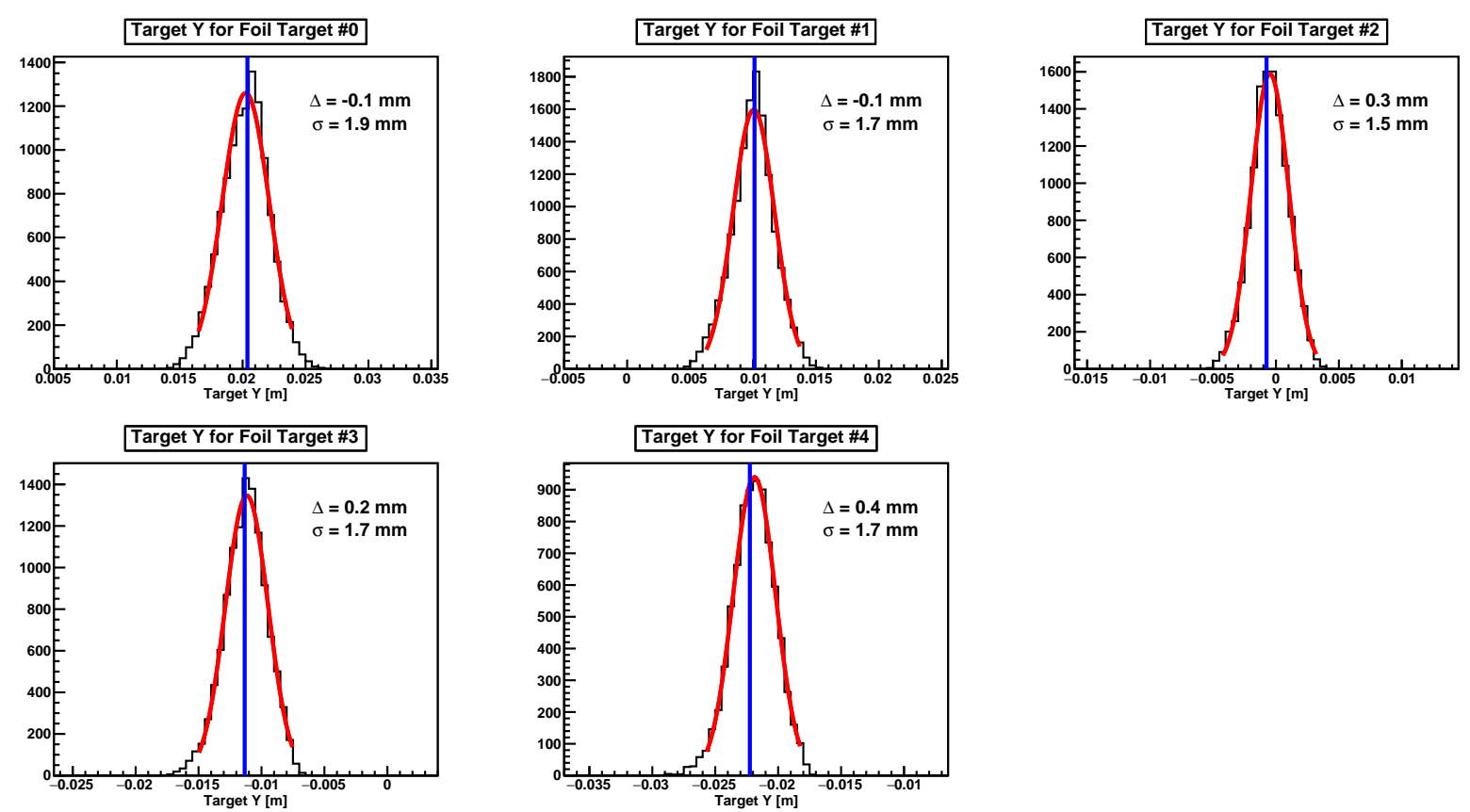

Figure 3.28. Target y distribution after optimization. The blue vertical lines indicate the true foil target positions and Delta is the difference between the data Gaussian fitting center and real position.

\subsubsection{Calibration of $x_{t a r}^{\prime}$ and $y_{t a r}^{\prime}$}

Figure 3.29 represents the sieve pattern for $1 \mathrm{GeV}$ after $x_{t a r}^{\prime}$ and $y_{\text {tar }}^{\prime}$ optimization. As the GMp sieve is 1 inch thick, the offset for the hole center to the average center distribution caused by the thickness of the sieve could not be neglected. The cross points are obtained by projecting the track to a plane in the middle of the front and back surfaces of the sieve. So the projected position should be centered at the nominal hole sieve position. Clearly, the reconstructed positions are matched well with the crosses. The Figure 3.30 gives more quantitative information about the accuracy of optimization, where the position distributions were projected to the horizontal and vertical direction. A Gaussian fit was made for the events of each sieve hole in each direction and the mean value of the Gaussian fit was compared to the corrected central position. The average of absolute discrepancy in the horizontal direction is $0.39 \mathrm{~mm}$ and in the vertical direction is about $0.78 \mathrm{~mm}$. 

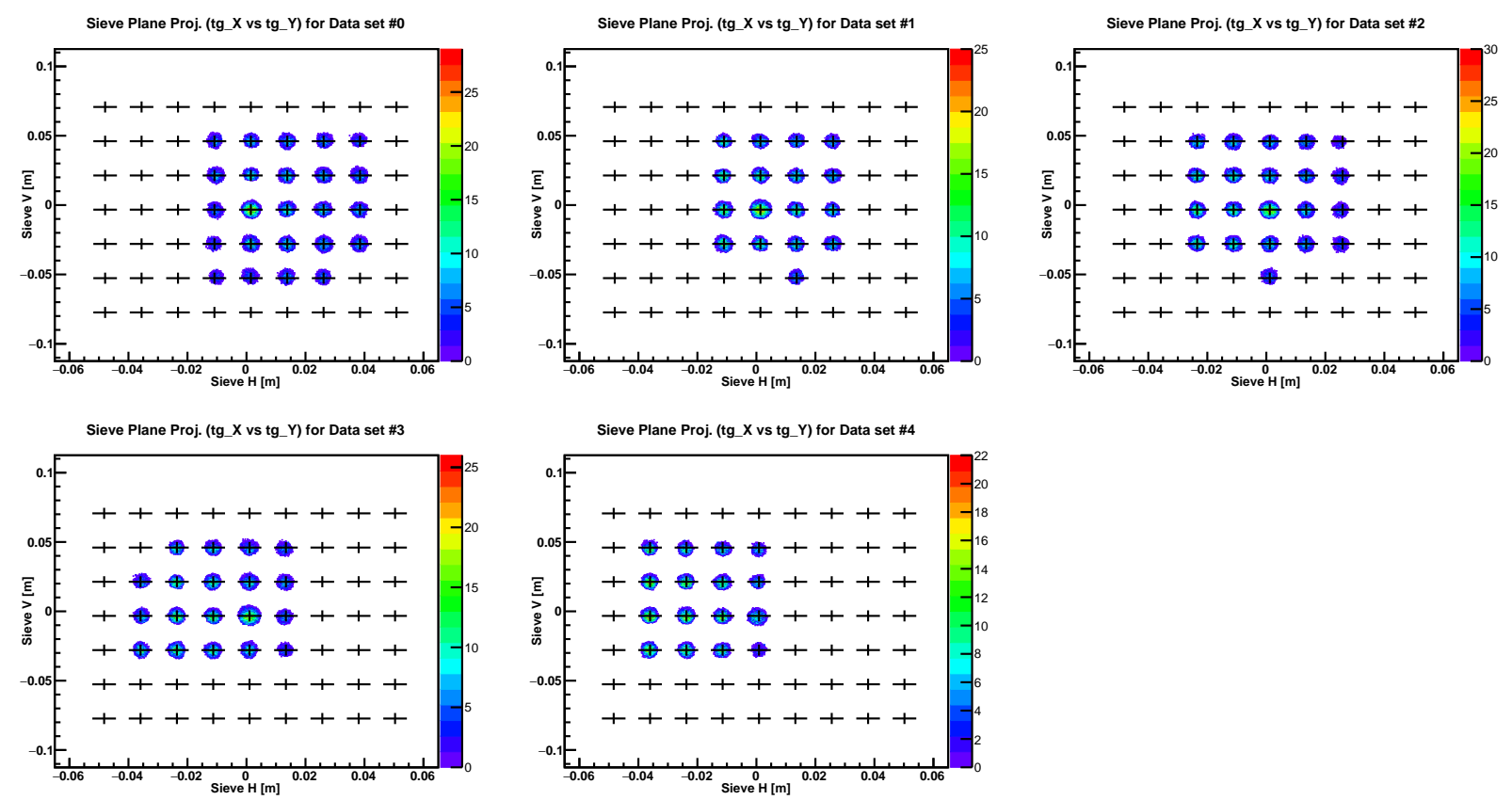

Figure 3.29. Optimized reconstructed sieve pattern for five foils of multi-foil carbon target. The crosses indicate the average of front and back hole centers.

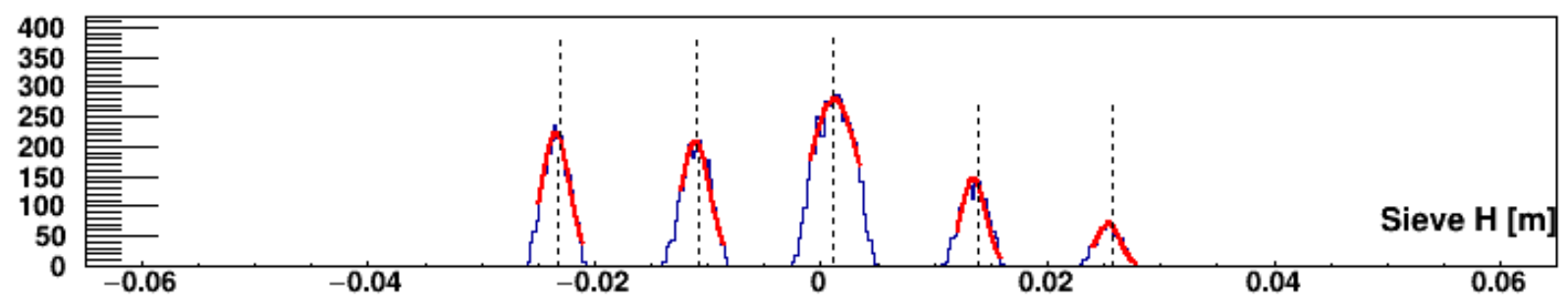

Figure 3.30. The position distribution in the horizontal direction with the Gaussian fitting of the one of the rows of sieve holes in the foil target.

\subsubsection{Momentum Calibration}

Figure 3.31 shows the optimization results for the left HRS Spring 2016 run period. The vertical red lines indicate the expected $\delta$ positions. The discrepancy between the mean value 
Dp_Kin for Delta Scan Kine. -4\%

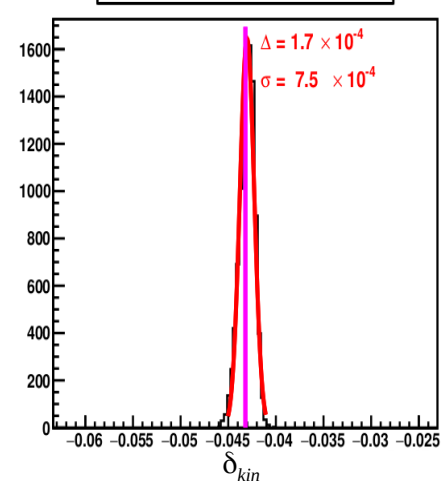

Dp_Kin for Delta Scan Kine. $2 \%$

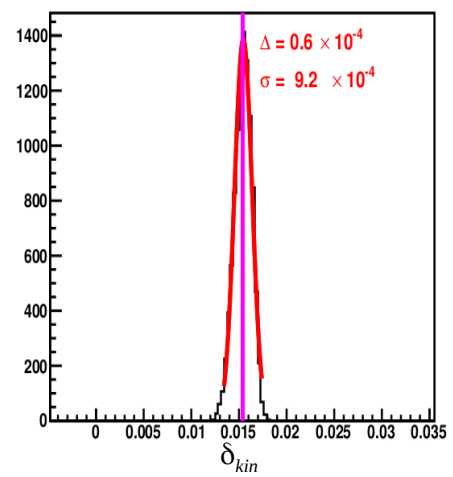

Dp_Kin for Delta Scan Kine. $-2 \%$

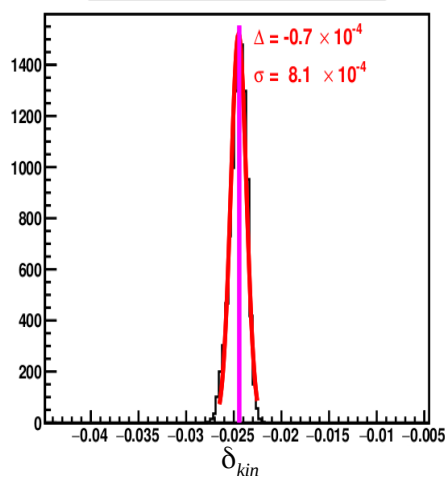

Dp_Kin for Delta Scan Kine. 4\%

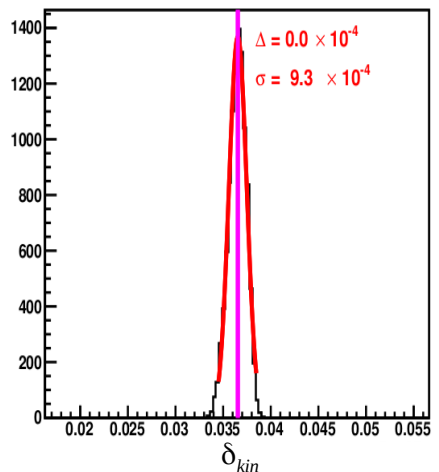

Dp_Kin for Delta Scan Kine. $0 \%$

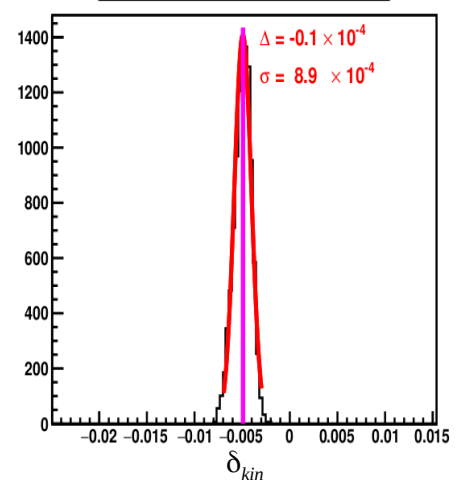

$$
\begin{gathered}
\delta_{\text {set }}=\frac{p_{\theta_{0}}-p_{0}}{p_{0}} \\
\delta_{\text {kin }}=\delta_{\text {rec }}-\frac{p_{\theta}-p_{\theta_{0}}}{p_{0}}
\end{gathered}
$$

Figure 3.31. The distribution of relative momentum for delta scan runs after optimization. The red line indicates the expected $\delta$ and $\Delta$ is the difference between reconstructed and expected $\delta$.

of the Gaussian fit to reconstructed delta and the set delta is:

$$
\delta_{k i n}-\delta_{s e t}=\delta_{r e c}-\frac{p_{\theta}-p_{0}}{p_{0}},
$$


where,

$$
\begin{aligned}
\delta_{s e t} & =\frac{p_{\theta_{0}}-p_{0}}{p_{0}} \\
\delta_{k i n} & =\delta_{r e c}-\frac{p_{\theta}-p_{\theta_{0}}}{p_{0}} .
\end{aligned}
$$

Here, $p_{0}$ is the set momentum, $p_{\theta 0}$ is the elastic peak at central angle and $p_{\theta}$ is elastic peak at an angle $\theta$. The momentum resolution for this period was estimated to be $1.5 \times 10^{-4}$.

\subsubsection{Check of Optics Across Angular Acceptance}

The study of the alignment of the invariant mass peak in two dimensional $y_{t a r}^{\prime}$ vs $x_{t a r}^{\prime}$ bins with new optics as shown in Fig. 3.32. Clearly, our optics reconstructs W better than $0.4 \mathrm{MeV}$ across the majority of the angular acceptance.

\section{Data: (W Peak - 938)(MeV)}

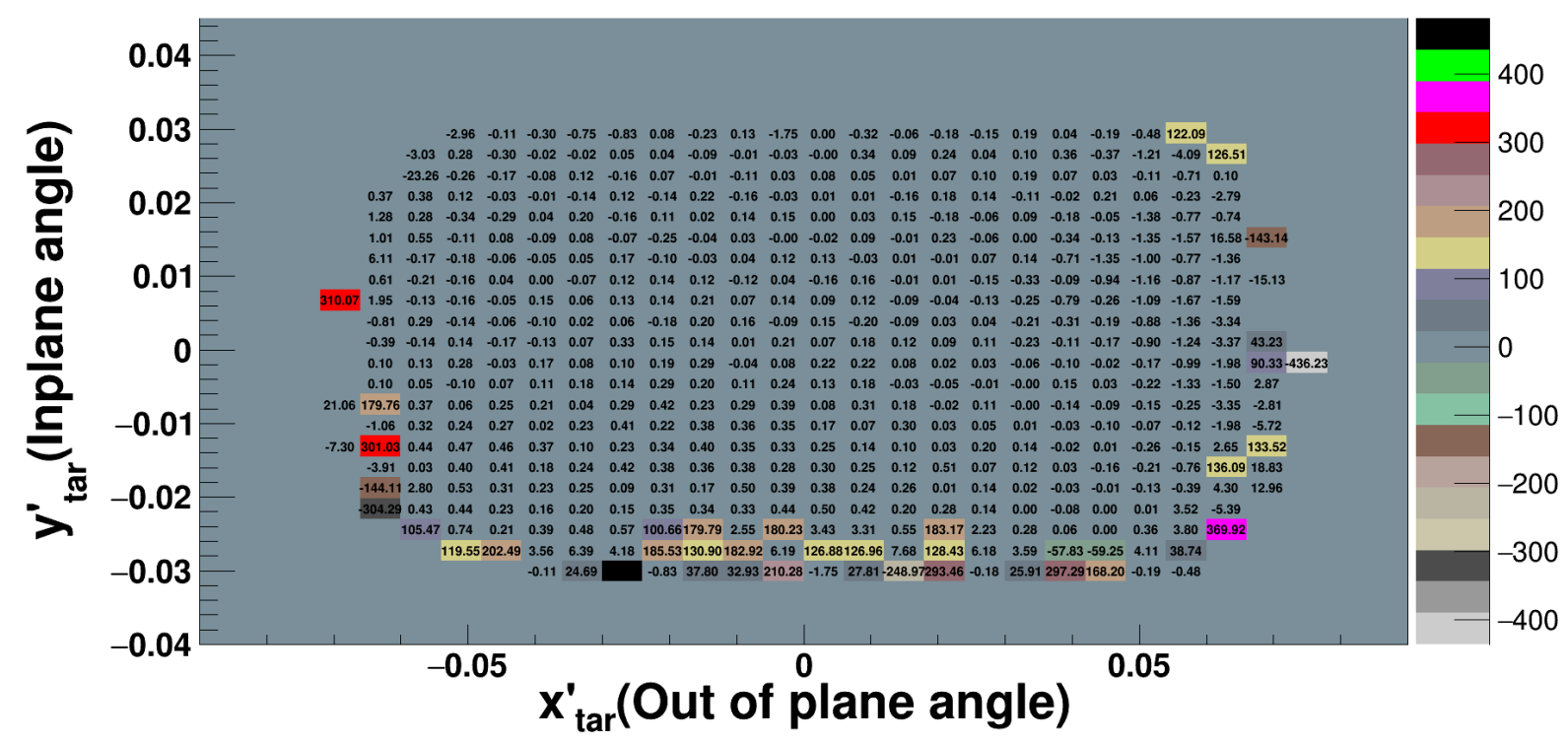

Figure 3.32. Reconstructed invariant mass shift at large $x_{t a r}^{\prime}$ for kinematics K1-1 with optimized optics. 


\subsection{Determination of Target and Spectrometer Offsets}

It is very important to have accurate knowledge of the scattering angle in order to have a precise measurement of the e-p elastic scattering cross sections. This requires an accurate knowledge of the target and spectrometer offsets. The geometry used to express the y target offset in terms of beam position and target $\mathrm{z}$ offset is shown in the figure 3.33. The relation between the target $\mathrm{z}$ offset relative to the hall center and target $\mathrm{y}$ offset is given by

$$
\Delta y \rightarrow \Delta y+s_{\text {off }}=\Delta x \cos \theta+\Delta z \sin \theta
$$

The target and beam position offsets are calculated by fitting the following equation:

Table 3.8

Target $y, x$ beam position and spectrometer pointing informations for surveyed GMp Fall 2016 kinematics.

\begin{tabular}{|c|c|c|c|c|}
\hline Kinematic & HRS & $\mathrm{y}_{\text {tar }}(\mathrm{mm})$ & $\mathrm{x}_{\text {beam }}(\mathrm{mm})$ & $\mathrm{s}_{\text {off }}(\mathrm{mm})$ \\
\hline K1-1 & LHRS & $-1.8348 \pm 0.0053$ & 0.5610 & 1.07 \\
\hline K3-6 & LHRS & $-1.0650 \pm 0.0012$ & 0.4655 & 0.385 \\
\hline K3-7 & LHRS & $-1.7468 \pm 0.003$ & 0.5173 & 0.658 \\
\hline K4-9 & LHRS & $-1.0276 \pm 0.0011$ & 0.6040 & 0.385 \\
\hline K4-11 & LHRS & $-1.9338 \pm 0.0014$ & 0.5892 & 1.07 \\
\hline K1-2 & RHRS & $-1.81994 \pm 0.0067$ & 0.5224 & 3.314 \\
\hline K4-12 & RHRS & $-1.8283 \pm 0.002$ & 0.5681 & 3.314 \\
\hline
\end{tabular}

$$
\left(\Delta y+s_{o f f}-\Delta y_{M C}\right) / \cos \theta-\Delta x_{B P M}=\Delta x_{\text {beamline }}+\Delta z \tan \theta
$$

where $s_{\text {off }}$ is the spectrometer offset and $\Delta x$ is the beam position offset which is the combination of beam position offset relative to the center of BPM and the beam offset relative 


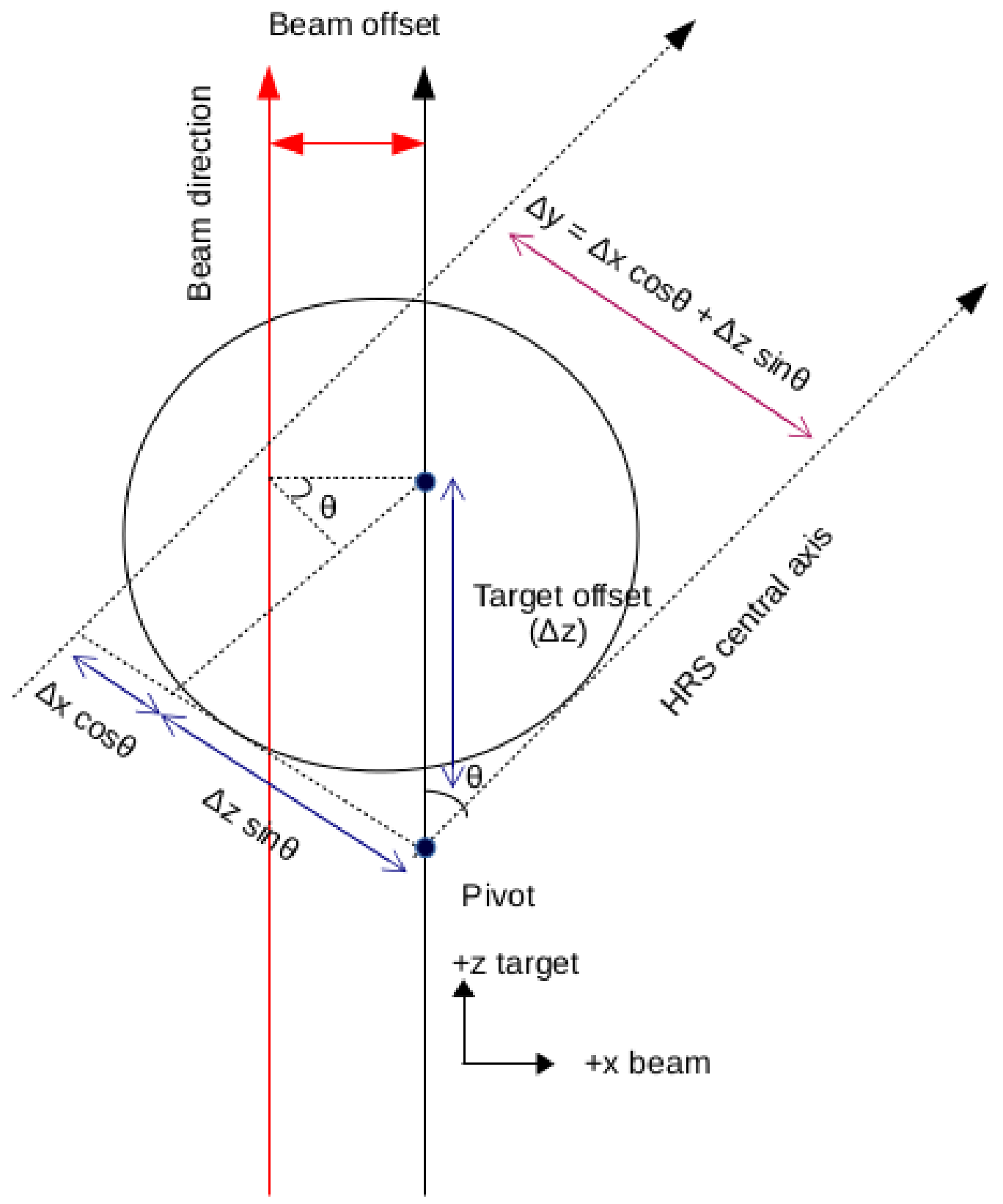

Figure 3.33. The geometry of the target and beam positions.

to the beamline i.e.

$$
\Delta x=\Delta x_{B P M}+\Delta x_{\text {beamline }} .
$$


Using equation 3.44 in equation 3.42 yields

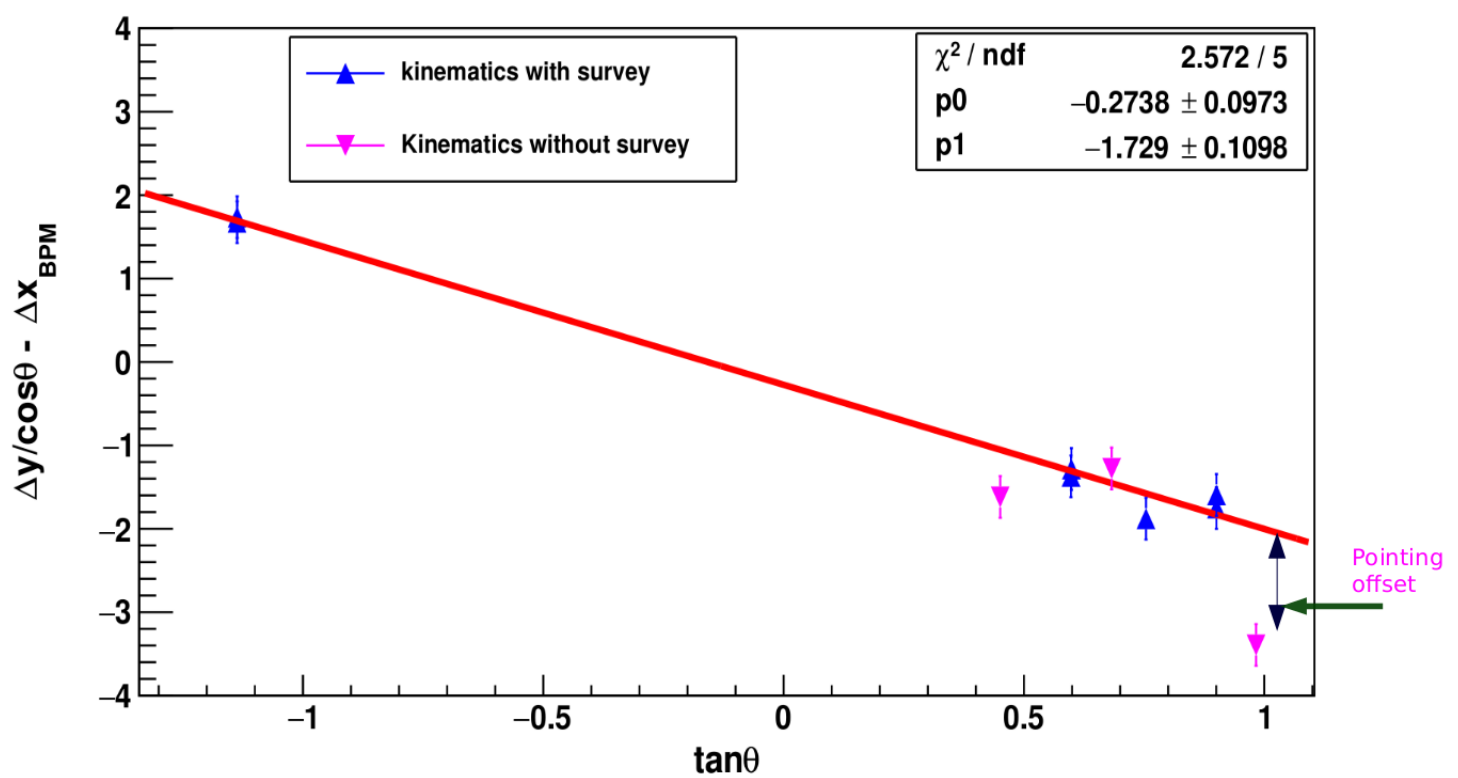

Figure 3.34. Determination of target $z$ and spectrometer offsets.

$$
\Delta y+s_{\text {off }}-\Delta y_{M C}=\left(\Delta x_{B P M}+\Delta x_{\text {beamline }}\right) \cos \theta+\Delta z \sin \theta
$$

where $\Delta y_{M C}$ is the offset in $y$ obtained from Monte Carlo keeping beam, target and spectrometer in ideal position. It can be seen from equation 3.43 that if one plot the quantity on left side vs $\tan \theta$ and fit linearly using the information from the table 3.8 for surveyed kinematics, the slope of the fit gives the $z_{\text {tar }}$ offset and the intercept gives the $\Delta x_{\text {beamline }}$. The fit results are shown in figure 3.34. Table 3.9 represents spectrometer pointing offsets for the kinematics with no survey obtained by calculating the y distance of the point from the fitted line yields the spectrometer pointing offsets for the kinematics with no survey. 
Table 3.9

Spectrometer pointing offsets for un-surveyed LHRS Fall 2016 kinematics.

\begin{tabular}{|c|c|c|}
\hline Kinematic & $\theta$ & $\mathrm{s}_{\text {off }}(\mathrm{mm})$ \\
\hline K3-4 & 24.25 & 0.562 \\
\hline K3-8 & 44.5 & 1.41 \\
\hline K4-10 & 34.4 & 1.41 \\
\hline
\end{tabular}

\subsection{SIMC Simulation}

SIMC [50] is a Standard Hall A and Hall C Monte Carlo simulation package that uses COSY [51] to transport particle trajectories through magnetic elements to simulate the elastic scattering process based on input variables such as beam, target, kinematic setting, HRS, and the final state. There are three main components of SIMC: the event generator, the spectrometer optical model and the checking of particle trajectories against a list of apertures. The event generator randomly generates the position and energy of the incident electron at the target taking into account the target length and beam rastering. This also includes the cross-section weighting and radiative corrections. Besides this, it also randomly generates the momenta and angles of the scattered electron with a flat distribution over limits exceeding the actual experimental spectrometer acceptance. The scattered events are then transported through the spectrometer after applying ionization energy loss and multiple scattering effects in the target material, chamber, and cells. Multiple scattering is applied in steps along the path from the target through the detector. This uses a small angle scattering approximation where the scattering angle follows a Gaussian distribution whose width in both dispersive and non dispersive directions given by

$$
\theta_{0}=\frac{13.6 \mathrm{MeV}}{\beta c p} \sqrt{x / X_{0}}\left(1+0.038 \ln \frac{1}{\beta^{2}} \frac{x}{X_{0}}\right)
$$


In the end, only the scattered electrons are transported through the spectrometer. In order to transport the electrons through the spectrometer, the spectrometer optics models in the COSY Infinity program are used. COSY generates both the forward and backward matrices, where the forward matrix is used to transport the particles from the spectrometer entrance window to the focal plane and the backward matrix is used reconstruct them back the particle to the target vertex. SIMC ensured that the particles have gone through each aperture by checking the acceptance of each aperture using a set of aperture cuts. These apertures include the front, middle and back aperture of each magnetic element in the QQDQ configuration. The aperture of the vacuum after Q3, the aperture of the rectangular collimator, and the aperture of each detector are added according to their actual size and positions. The events which are accepted will have their focal-plane positions and angles determined by a tracking detector which can be supplied with a position resolution.

In order to study the spectrometer acceptance and reconstruction, we use a uniform generator, where the important generated target variables are: $x_{t a r}, x_{t a r}^{\prime}, y_{t a r}^{\prime}, y_{t a r}$ and $\delta$ ,the focal plane variables in transport coordinate system are: $x_{f p}, y_{f p}, x_{t a r}^{\prime}$ and $y_{t a r}^{\prime}$ and the reconstructed variables are: $x_{t a r}^{\prime}, y_{t a r}^{\prime}, y_{t a r}$ and $\delta$. The variable $x_{t a r}$ is not reconstructed and needs to be determined by other means.

\subsection{Radiative Correction}

In Born approximation, the electron-proton differential scattering cross section was derived to lowest order in the electromagnetic coupling constant $(\alpha)$. This includes only the amplitude due to the exchange of a single virtual photon between the incident electron and the proton as shown in fig(a) of Fig. 3.37. However, the highest order processes in $\alpha$ also affect the cross sections. So, to compare the measured cross section directly to the theoretical prediction, it is very important to calculate the radiative processes from the data. Emission of radiation can occur before or after scattering, which is indicated in the Fig. 3.36 by initial or final state emission. The circle represents a zoomed in scattering vertex. Points A and 

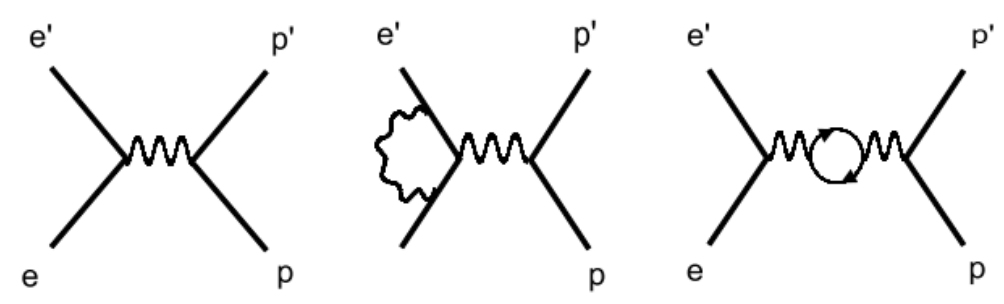
a) Born term
b) vertex
c) vacuum
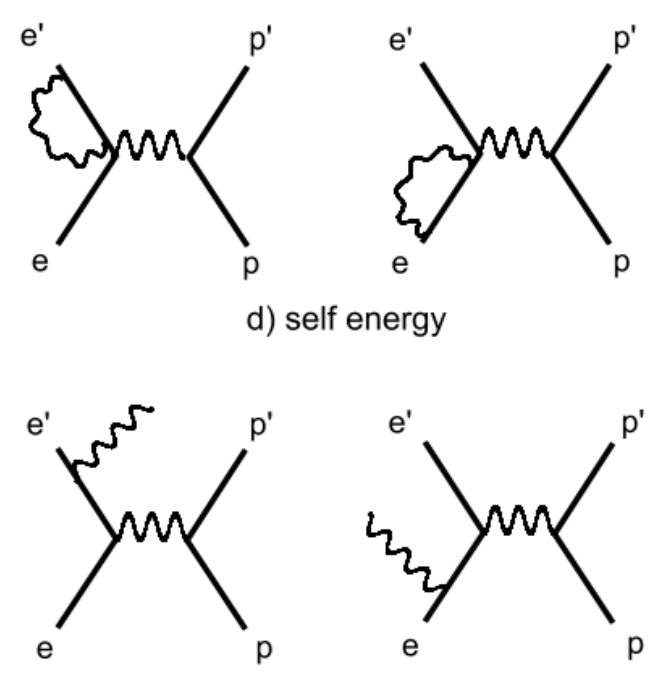

e) Bremsstrahlung

Figure 3.35. Feynman diagrams for the elastic e-p scattering including the Born and lowest order radiative correction graphs [15].

A' represent points infinitesimally close to the scattering vertex before and after scattering. Emission of real or virtual photons reduces the energy of the electron in the target medium. This radiation can be emitted due to:

1. Internal corrections: The internal effects take place at the scattering vertex includes internal bremsstrahlung, vacuum polarization, vertex processes, and two-photon exchange. In this process, real photons are emitted when a charged particle involved in the reaction is accelerated by the field of the nucleus involved in the primary e- 


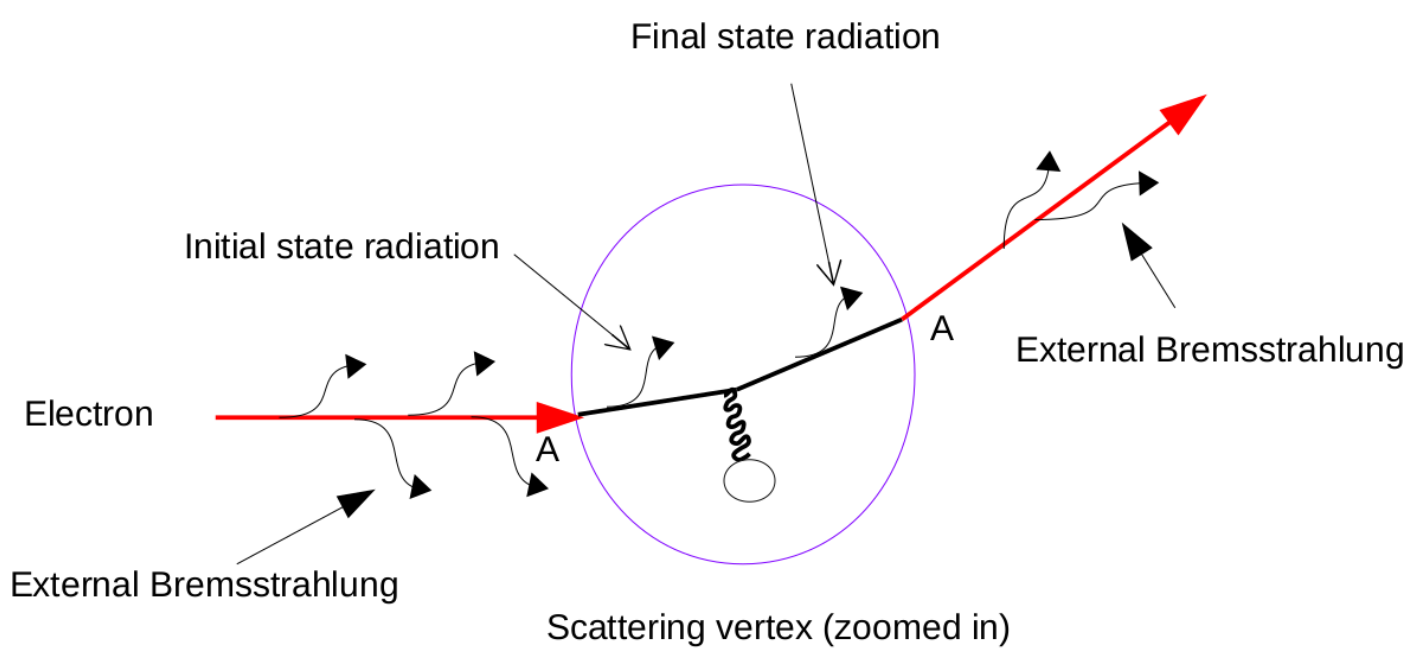

Figure 3.36. Schematic of radiative energy loss in elastic electron-proton scattering

p scattering. The emission of initial state radiation affects the $Q^{2}$ whereas the final state radiation reduces the total available energy after scattering, thereby changing the acceptance of the scattered electron in the detectors.

2. External bremsstrahlung: In this process, the electron radiates while it interacts with the Coulomb field of a nucleus other than the one involved in the e-p scattering. This process can be caused by bremsstrahlung in material that the electron passes through before and after the scattering.

Radiative processes depend on the target material, geometry and kinematics of the scattering. They modify data cross section and the kinematics of the incident and final state of the electron and proton. The emission of a real photon causes the particle's momentum to be different from the actual momentum at the scattering vertex whereas the emission of additional particles such as TPE processes affects only the magnitude of the measured cross section. These processes change the elastic cross section form $\delta$ function smeared by detector resolution to an asymmetric peak with an extended elastic tail at lower energies. This tail extends down to the values of scattered momentum where other processes such as 

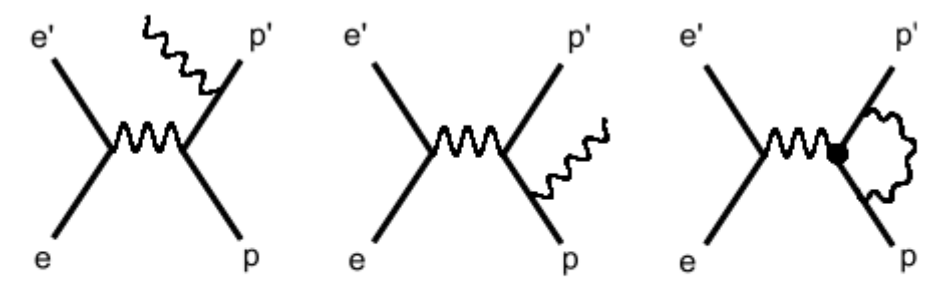

a) bremsstrahlung

b) vertex
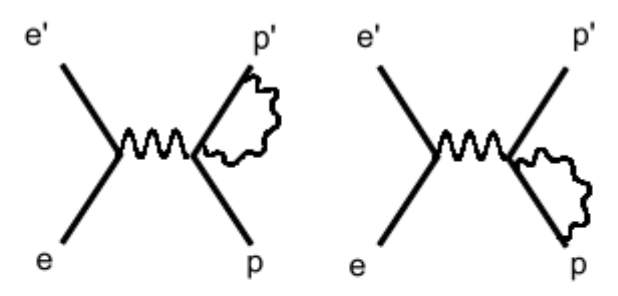

c) self energy
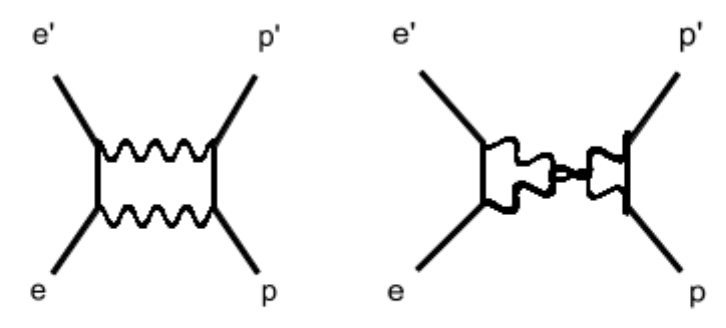

d) two-photon

Figure 3.37. Feynman diagrams for the elastic e-p scattering including lowest order radiative correction graphs [15].

$\pi$ production and $\Delta$ resonance occur.

The radiative correction formalism was first calculated by Mo and Tsai [28] and improved by Walker [52] and Ent [53]. The conversion of the measured cross section to the one-photon exchange cross section is given by:

$$
\frac{d \sigma}{d \Omega}^{\text {measured }}=\left(1+\delta_{\text {corr }}\right) \frac{d \sigma}{d \Omega}^{\text {Born }}
$$


where

$$
\delta_{c o r r}=\delta_{i n t}+\delta_{i n t}^{\prime}+\delta_{e x t}
$$

Here, $\delta_{i n t}$ and $\delta_{\text {ext }}$ represent the internal and external radiative corrections, respectively, whereas $\delta_{i n t}^{\prime}$ results from the improvements to the internal corrections by Walker [52]. The higher order corrections are obtained by exponentiating $\delta_{\text {int }}$ :

$$
{\frac{d \sigma^{\text {measured }}}{d \Omega}}^{\text {morr }} \frac{d \sigma}{d \Omega}^{\text {Born }}
$$

This approximation is mainly valid only for infrared divergent terms and the error caused in the non-divergent terms is estimated to be less than $0.7 \%$. The contribution from vacuum polarization and the electron vertex are calculated exactly whereas the contributions from TPE are limited to the infrared divergent parts. Based on the modification made by Walker [52] and Ent [53] to the work of Mo and Tsai [28], the internal radiative correction can be written as

$$
\delta_{\text {int }}=-\frac{\alpha}{\pi}\left(\frac{28}{9}-\frac{13}{6} \ln \left(\frac{Q^{2}}{m_{e}^{2}}\right)+\delta_{\text {int.brem }}\right)
$$

The internal bremsstrahlung contributions are calculated under the assumption by Tsai [54] that $\Delta E\left(1+\frac{2 E}{M_{p}}\right) \leq E^{\prime}$. Here, $E$ and $E^{\prime}$ are the incident and scattered electron energy respectively. $\Delta E=E_{\text {elastic }}-E_{\text {cutoff }}$ is the $E^{\prime}$ cutoff of the elastic peak.

Walker [52] improved the precision to the original radiative correction by adding a term $\delta_{\text {int }}^{\prime}$. This involve correction for the $\Delta E\left(1+\frac{2 E}{M_{p}}\right) \leq E^{\prime}$ approximation made by Tsai to the internal bremsstrahlung, addition of the $q \bar{q}$ and $\mu^{+} \mu^{-}$contributions to the vacuum polarization or loop diagrams which were not taken into account before as well as the correction for a sign error in Tsai's paper [54] for the Schwinger's correction [55] to the noninfrared divergent part of the soft photon emission cross section. The corrections that are applied to the cross sections due to bremsstrahlung in the target material and the effects of ionization energy loss 
are called external corrections. In terms of the electron cross section the external corrections $\delta_{\text {ext }}$ are expressed as:

$$
e^{\delta_{\text {ext }}}=\left(\left(\frac{d \sigma(E)}{d \Omega}\right)^{-1}\left[L T_{\text {corr }} \int_{E_{\text {elastic }}-\Delta E^{\prime}}^{E_{\text {elastic }}} \frac{d^{2} \sigma\left(E^{\prime}, E\right)}{d \Omega d E^{\prime}} d E^{\prime}\right]\right.
$$

where $L T_{\text {corr }}$ is the correction for the Landau tail as calculated from the Landau distribution and the cross section of an electron of initial energy $E$ and final energy $E^{\prime}$, where the electron is emitting bremsstrahlung radiation with $t_{i}$ and $t_{f}$ radiation lengths of material before and after the scattering is

$$
\begin{aligned}
\frac{d^{2} \sigma\left(E, E^{\prime}\right)}{d \Omega d E^{\prime}} & =\left(\frac{R \Delta E^{\prime}}{E}\right)^{b_{i} t_{i}}\left(\frac{\Delta E^{\prime}}{E_{\text {elastic }}}\right)^{b_{i} t_{i}} \frac{1}{\left(1+b_{i} t_{i}\right)} \frac{1}{\left(1+b_{f} t_{f}\right)} \\
& \times\left[\frac{d \sigma(E)}{d \Omega} \frac{b_{f} t_{f}}{\Delta E^{\prime}} \phi\left(\frac{\Delta E^{\prime}}{E_{\text {elastic }}}\right)+\frac{d \sigma\left(E-R \Delta E^{\prime}\right)}{d \Omega} \frac{b_{i} t_{i}}{\Delta E^{\prime}} \frac{R \Delta E^{\prime}}{E}\right]
\end{aligned}
$$

Here, the parameter $R$ represents the recoil of the proton and is about $\left(\frac{E}{E^{\prime}}\right)^{2}$, the function $\phi\left(\frac{\Delta E^{\prime}}{E}\right)=\phi\left(\frac{\omega}{E}\right)$ gives the shape of the bremsstrahlung spectrum which is equal to one at $\omega / E=0$ where $\omega$ is the lost energy of the electron after passing a thickness of $t$ radiation lengths and the parameter $b \sim 3 / 4$.

The radiative correction in SIMC was implemented as described in Ref. $[53,55,56]$. As SIMC simulates events in coincidence mode, both the electron and recoiling proton can be responsible for the emission of photon. So, integration over all final states of scattered proton must not be done in the inclusive scattering.

\subsection{Ionization Loss}

The mean energy loss obtained from the Bethe-Bloch formula may be fine for bulk deposition but it is inadequate in describing the energy loss of single particles [57]. There are large statistical fluctuations in the distribution of energy loss $d E / d x$ due to a small number 
of collisions involving very energetic electrons. So for detectors of moderate thickness $x$, the energy loss probability distribution $f(\Delta ; \beta \gamma, x)$ is sufficiently described by the highly-skewed Landau-Vavilov distribution. The most probable energy loss is given by:

$$
\Delta_{p}=\xi\left[\ln \frac{2 m c^{2} \beta^{2} \gamma^{2}}{I}+\ln \frac{\xi}{I}+j-\beta^{2}-\delta(\beta \gamma)\right]
$$

Here, $I$ is mean excitation energy in $\mathrm{eV}, \delta(\beta \gamma)$ is the density effect correction to ionization energy loss, $\xi=(K / 2)<Z / A>z^{2}\left(x / \beta^{2}\right) \mathrm{MeV}$ for a detector of a thickness $x$ in $\mathrm{gcm}^{-2}$ and $j=0.2$. As the Landau distribution is an asymmetric distribution with a long tail at the high energy side, its most probable value is different from its average value. The most probable value is simply the value at which the distribution has a maximum given the skewed distribution. In this experiment, for incident electrons the energy loss is subtracted from the nominal beam energy whereas for scattered electrons the loss is added to the measured electron momentum. More detail description of analysis of energy loss is presented in Ref. [58].

\subsection{HRS Optics with Saturated Q1}

As noted in the Section 3.7, prior to the Fall 2016 run the first superconducting quadrupole in the left HRS was replaced with a resistive magnet. The magnetic field of this new magnet was found to saturate due to the iron core above a momentum of $\approx 3 \mathrm{GeV}$. Due to time constraints the experiment took optics data only for the nominal tune. Additionally, the magnet was set based on current assuming a linear dependence of the field integral ( $\left.\int \mathrm{Bdl}\right)$ on the current (i.e. the field saturation was not corrected for). Therefore, when using the nominal reconstruction matrix there was a mismatch between the reconstruction and the forward tune. This is illustrated in Fig. 3.38 for the kinematic setting with the largest saturation (K3-4) at $3.962 \mathrm{GeV} / \mathrm{c}$. During data taking, the field probe indicated that the field was saturated by about $7 \%$ at this kinematic setting which is simulated in the MC. Shown 

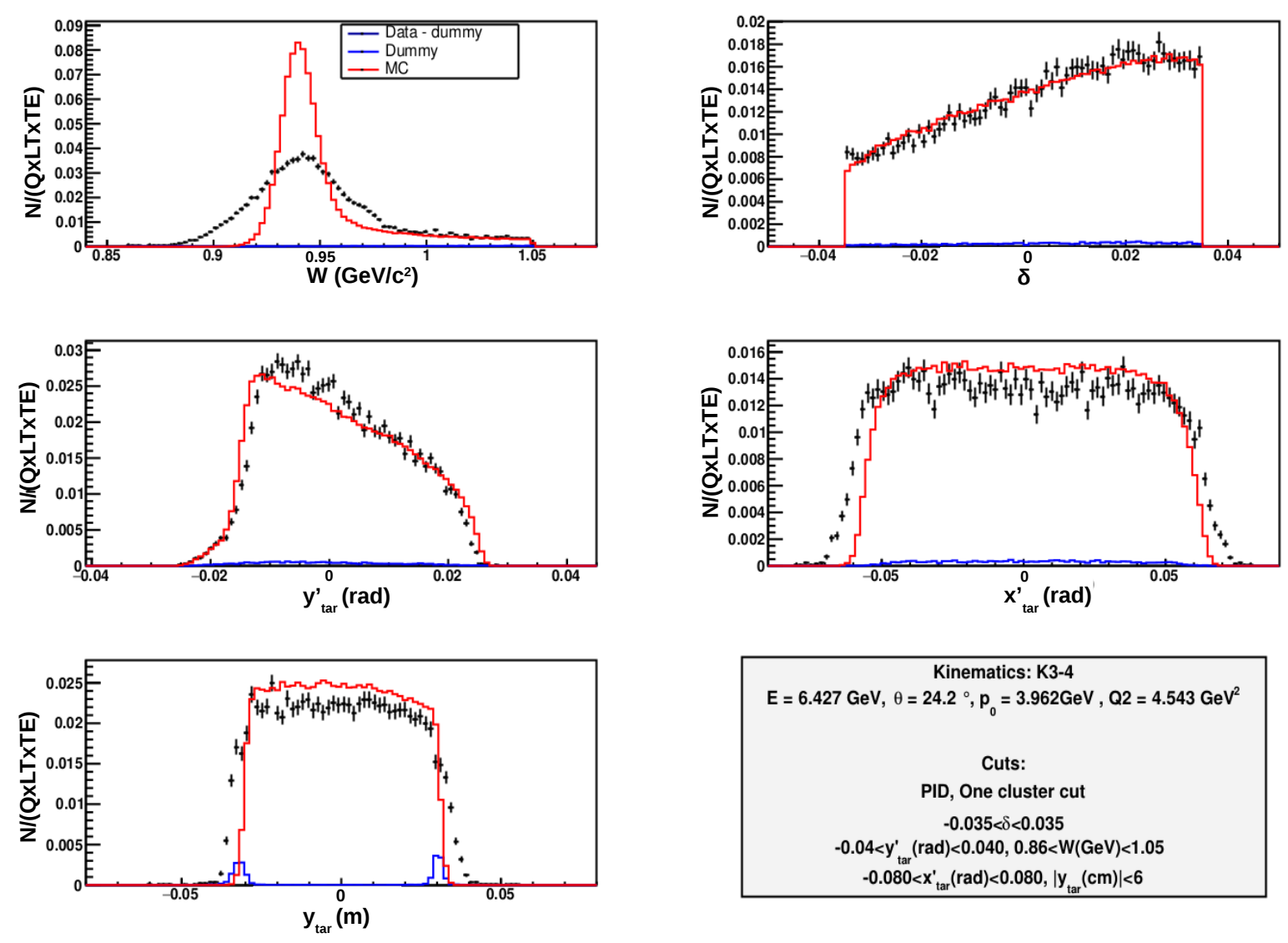

Figure 3.38. Data vs MC comparison plots of target quantities for kinematics K3-4 with nominal tune. The red histograms represents MC, black distributions represents background subtracted data and blue histogram represents the background.

in this plot is the nominal MC (with no Q1 saturation included) compared to the data. The poor resolution in $\mathrm{W}$ for the data, and the significant discrepancies between data and MC, is due to the inconsistency in the field ratios of the magnet in MC with respect to data. The effect on the MC of having a forward field ratio changed and keeping reconstruction matrix element the same is shown in top right plot of Fig. 3.39 where the MC now exhibits the same poor $\mathrm{W}$ resolution as the data. In order to fix the data reconstruction in the absence of optics data for each saturated tune, the following steps were implemented:

1. determine accurately what the field saturation factor was, 
2. develop a procedure to correct the data reconstruction matrix elements for this mismatch.
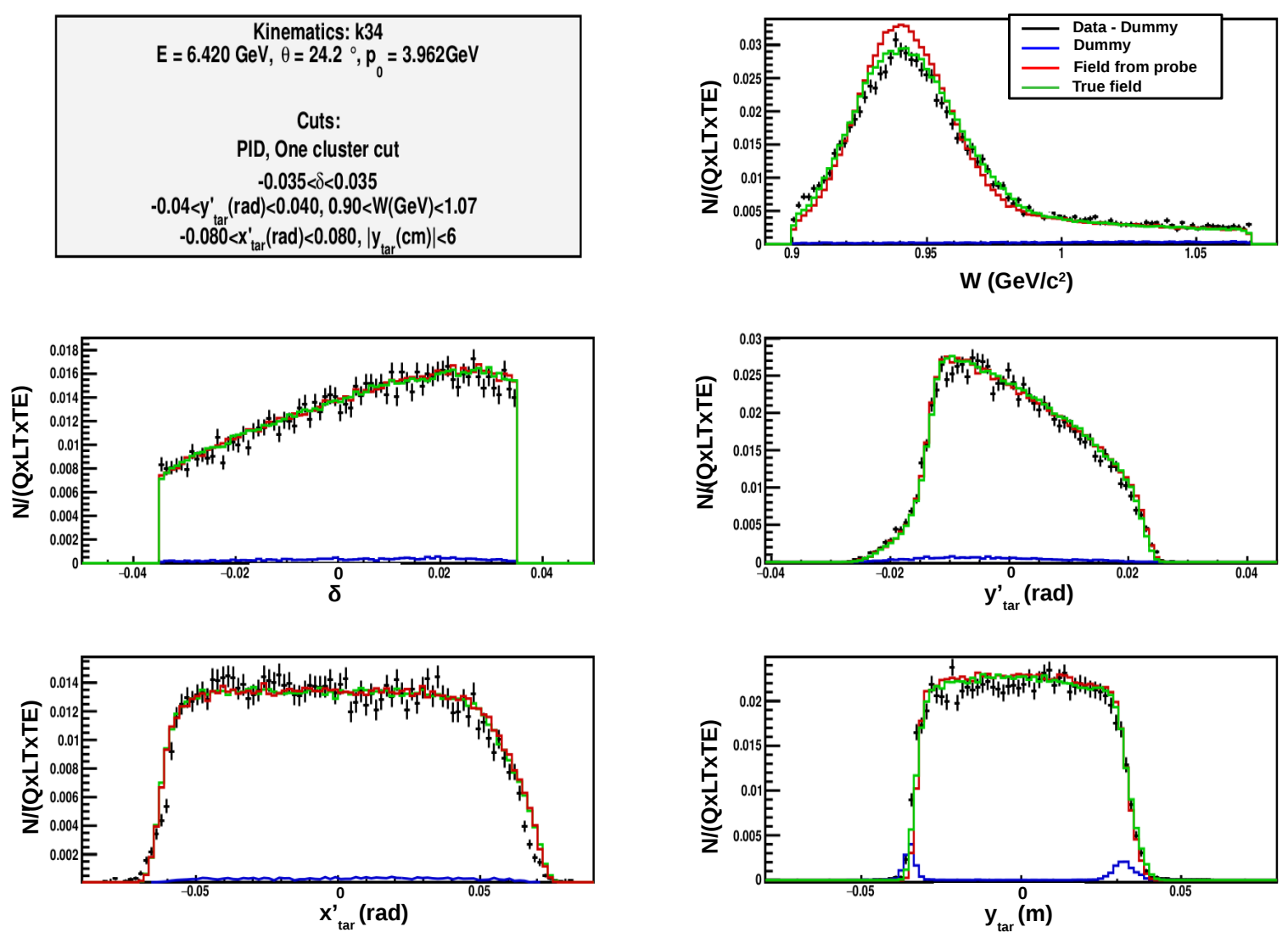

Figure 3.39. Data vs MC comparison plots for K3-4 kinematics with optimized forward tunes in red. The black histogram represents background subtracted data, green histogram represents MC for nominal tune and blue histogram represents background.

The fractional reduction of the field of the resistive SOS quad as a function of the readback current is shown in the Fig. 3.40. This shows the reduction in the field integral relative to the nominal tune due to the Q1 saturation above a set current of 550 A. The Table 3.10 shows the status of the Fall 2016 kinematics with field/momentum determined from the field probe (hereafter referred to as the nominal tune) for both DVCS and GMp kinematics. The 
relative field per unit momentum for the saturated and linear case can be parametrized as:

$$
\left(\frac{B}{P}\right)_{\text {saturated }} /\left(\frac{B}{P}\right)_{\text {linear }}=1-0.27\left[\frac{I_{\text {readback }}}{550 A}-1\right]^{2}
$$

Table 3.10

Status of the Q1 field for Fall 2016 kinematics where the relative fields are shown in the last column.

\begin{tabular}{|l|l|l|l|l|l|}
\hline Kinematics & $\theta$ & Set p0 (GeV/c) & $\begin{array}{l}\text { Read-back } \\
\text { Current }(\mathrm{A})\end{array}$ & $\begin{array}{l}\text { Hall probe } \\
\mathrm{B}(\mathrm{T})\end{array}$ & $\begin{array}{l}\text { Relative } \\
(\mathrm{B} / \mathrm{p})\end{array}$ \\
\hline \multicolumn{6}{|c|}{ DVCS } \\
\hline 362 & 20.99 & 3.187 & 664.704 & 1.09 & 0.98 \\
\hline 363 & 18.68 & 3.99 & 833.88 & 1.29 & 0.93 \\
\hline 601 & 25.56 & 3.59 & 749.60 & 1.20 & 0.96 \\
\hline 603 & 29.00 & 3.15 & 657.83 & 1.08 & 0.98 \\
\hline \multicolumn{5}{|c|}{ GMp } \\
\hline Optics & 17.51 & 1.08 & 225.25 & 0.375 & 1.000 \\
\hline K3-4 & 24.25 & 3.96 & 826.35 & 1.287 & 0.934 \\
\hline K3-6 & 30.91 & 3.22 & 672.41 & 1.104 & 0.984 \\
\hline K3-7 & 37.00 & 2.67 & 557.29 & 0.930 & 1.002 \\
\hline K3-8 & 44.50 & 2.15 & 447.38 & 0.748 & 1.003 \\
\hline K4-9 & 30.91 & 3.69 & 768.57 & 1.226 & 0.956 \\
\hline K4-10 & 34.40 & 3.26 & 679.72 & 1.113 & 0.982 \\
\hline K4-11 & 42.00 & 2.53 & 527.88 & 0.881 & 1.001 \\
\hline
\end{tabular}

After some investigation it was found that the reduction in $\int \mathrm{Bdl}$ was due to having moved the probe to the other side of the magnet to avoid conflicts due to the original mount being on the beam side after moving the Q1 to the LRHS. To determine the true $\int \mathrm{Bdl}$ reduction, the correlation between z-vertex and $y_{\text {tar }}^{\prime}$ (which should not be correlated) were fitted and compared to the expected value from MC for the kinematics with saturation. Dummy aluminum target was used for this study. Kinematic K3-8 was an unsaturated configuration below 550 A current and was used as a baseline for our analysis. Then the weighted average of the downstream and upstream foil slopes after subtracting the baseline slope in both data 


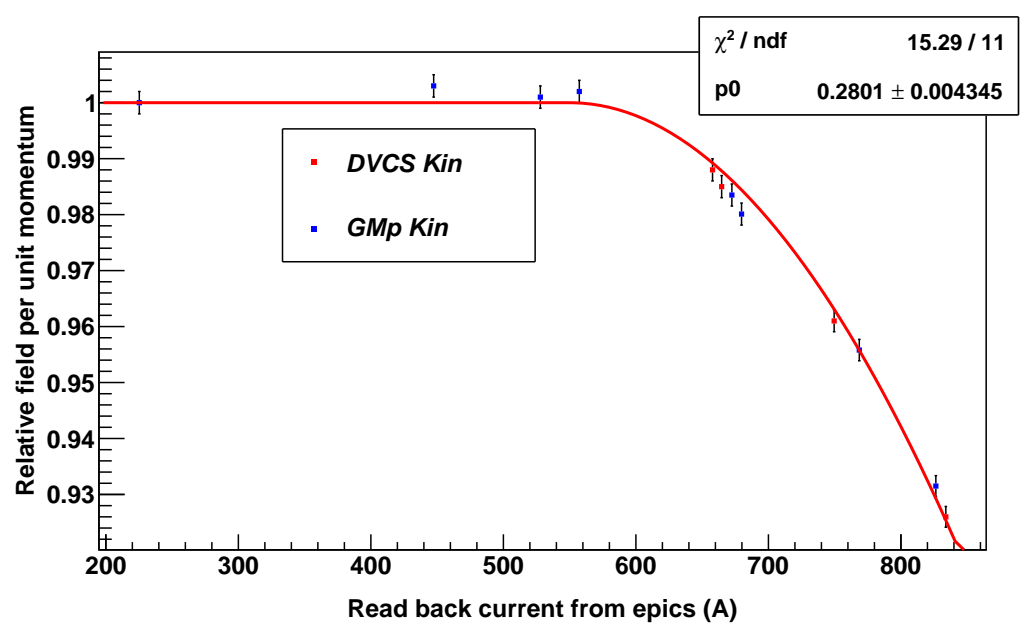

Figure 3.40. Relative field per unit momentum versus read back current showing saturation above the set current of $550 \mathrm{~A}$.

and $\mathrm{MC}$ were calculated. The ratio of the weighted average slope between data and MC is then multiplied with $\int \mathrm{Bdl}$ obtained from probe to determine the true $\int \mathrm{Bdl}$. The Table 3.11 shows the actual $\int \mathrm{Bdl}$ reduction for Q1 determined for each of the saturated kinematics.

Table 3.11

Determination of true $\int B d l$ reduction from dummy target z-vertex study.

\begin{tabular}{|l|l|l|l|l|l|l|}
\hline Kinematic & $\begin{array}{l}\text { Q1 } \\
\text { read- } \\
\text { back } \\
\text { current } \\
\text { (A) }\end{array}$ & $\begin{array}{l}\text { Factor } \\
\text { used in } \\
\text { MC }\end{array}$ & $\begin{array}{l}\text { fBdl re- } \\
\text { duction } \\
\text { field probe } \\
(\%)\end{array}$ & $\begin{array}{l}\text { (Data/MC) } \\
\text { slope }\end{array}$ & $\begin{array}{l}\text { True } \\
\text { Bdl } \\
\text { reduc- } \\
\text { tion(\%) }\end{array}$ & $\begin{array}{l}\text { Factor to be } \\
\text { used in MC } \\
\text { true) }\end{array}$ \\
\hline K3-4 & 826.35 & 0.934 & 6.60 & $1.037 \pm 0.09$ & 6.843 & $0.932 \pm 0.008$ \\
\hline K3-6 & 672.49 & 0.984 & 1.60 & $1.034 \pm 0.01$ & 1.654 & $0.984 \pm 0.009$ \\
\hline K4-9 & 768.59 & 0.956 & 4.40 & $1.005 \pm 0.01$ & 4.096 & $0.956 \pm 0.009$ \\
\hline K4-10 & 679.72 & 0.982 & 1.80 & $1.108 \pm 0.01$ & 1.994 & $0.980 \pm 0.008$ \\
\hline
\end{tabular}

where true $\int \mathrm{Bdl}$ reduction is the product of $\int \mathrm{Bdl}$ in $\mathrm{MC} *(\text { Data/MC) })_{\text {slope }}$. The Table 3.12 shows the true slope ratio between data and $\mathrm{MC}$ after using true $\int \mathrm{Bdl}$ in $\mathrm{MC}$. 

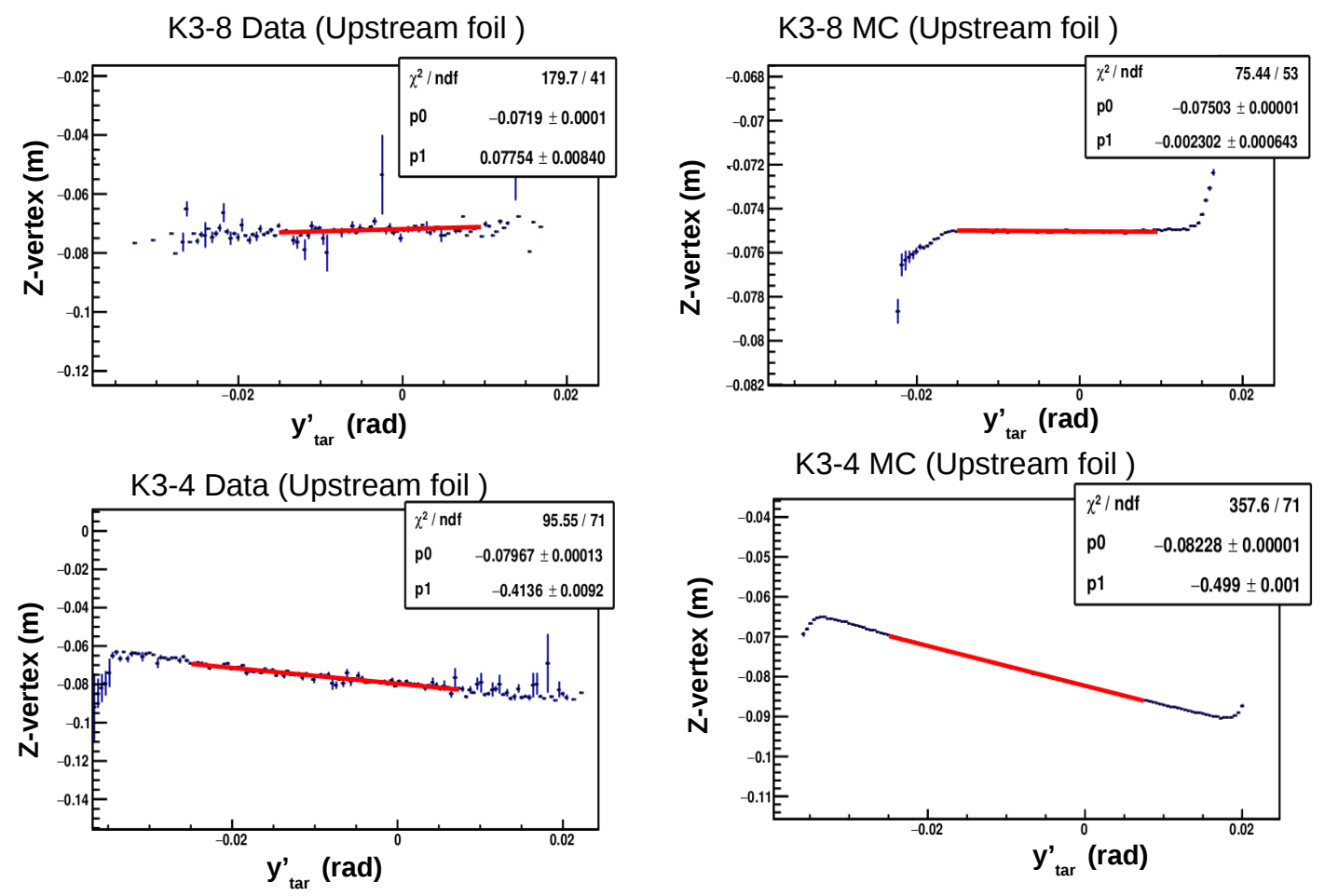

Figure 3.41. Target Z-vertex versus $y_{\text {tar }}^{\prime}$ used to estimate the true $\int B d l$ reduction.

Table 3.12

Data to $\mathrm{MC}$ slope ratio after using true $\int \mathrm{Bdl}$ in $\mathrm{MC}$

\begin{tabular}{|l|l|}
\hline Kinematic & $(\text { Data/MC })_{\text {slope }}$ \\
\hline K3-4 & 0.99 \\
\hline K3-6 & 0.99 \\
\hline K3-4 & 1.00 \\
\hline K3-4 & 0.99 \\
\hline
\end{tabular}

After determining the true Q1 field, the main challenge was to determine the optics for the Q1 saturated data as the experiment only took quality optics with the nominal tune. The MC uniform generator was utilized to get the correction factor due to saturation. The 
correction matrices were determined as follows:

$$
X_{t a r}^{\prime s a t}\left(x_{f p}, x_{f p}^{\prime}, y_{f p}, y_{f p}^{\prime}\right)=X_{t a r}^{\prime N o m i n a l}\left(x_{f p}, x_{f p}^{\prime}, y_{f p}, y_{f p}^{\prime}\right)+\Delta X_{t a r}^{\prime}\left(x_{f p}, x_{f p}^{\prime}, y_{f p}, y_{f p}^{\prime}\right)
$$

where $\Delta X_{t a r}^{\prime}$ is calculated by using MC as a difference of mis-tuned and nominal reconstruction matrix elements from optics model. For example for $X_{t a r}^{\prime}$ matrix:

$$
\begin{aligned}
X_{\text {tar }}^{\text {Nominal }} & =A_{0000}+A_{1000} x_{f p}+A_{0100} x_{f p}^{\prime}{ }^{2}+A_{1100} x_{f p} x_{f p}^{\prime} \\
X_{\text {tar }}^{\prime \text { Saturated }} & =B_{0000}+B_{1000} x_{f p}+B_{0100} x_{f p}^{\prime}{ }^{2}+B_{1100} x_{f p} x_{f p}^{\prime}
\end{aligned}
$$

So, the correction factor will be

$$
\Delta X_{t a r}^{\prime}\left(x_{f p}, x_{f p}^{\prime}, y_{f p}, y_{f p}^{\prime}\right)=B_{0000}-A_{0000}+\left(B_{1000}-A_{1000}\right) x_{f p}+\left(B_{1100}-A_{1100}\right) x_{f p} x_{f p}^{\prime}+\ldots
$$

where the correction factors are determined to order by order in target variable expansion and must use the same order as in data for consistent expansion. But Hall A Analyzer uses the different format from COSY and rotated coordinates at detectors. It was easier to simply use the COSY Based Monte Carlo to generate optics pseudo-data for Sieve slit and $e-p$ elastic delta scans. Then one can simply use the existing data optimization code to determine the reconstruction matrices in the same format as the Analyzer. Uniform MC sieve slit events were generated using the model of the Spring 2016 sieve slit for both nominal as well as saturated MC events for target y and angle calibration. On the other hand, simulated elastic events were used for momentum calibration, and then fitted both MC in the same way as data. The plot on the left side of the Fig. 3.42 shows the $\mathrm{W}$ distribution before and after correction to saturation and left shows the $y_{\text {tar }}$ distribution for the same case. Clearly, the resolution of both $y_{t a r}$ and $\mathrm{W}$ distributions improved significantly.

Fig. 3.43 shows data vs MC comparison of target quantities with $\int \mathrm{Bdl} \sim 0.9315 \mathrm{Tm}$ considered as a baseline tune estimated form z-vertex vs $y_{\text {tar }}^{\prime}$ correlation. Comparing the comparison plots with the nominal tune, there is a significant improvement across all reconstructed target quantities. Looking at the W distribution there is still some discrepancy 

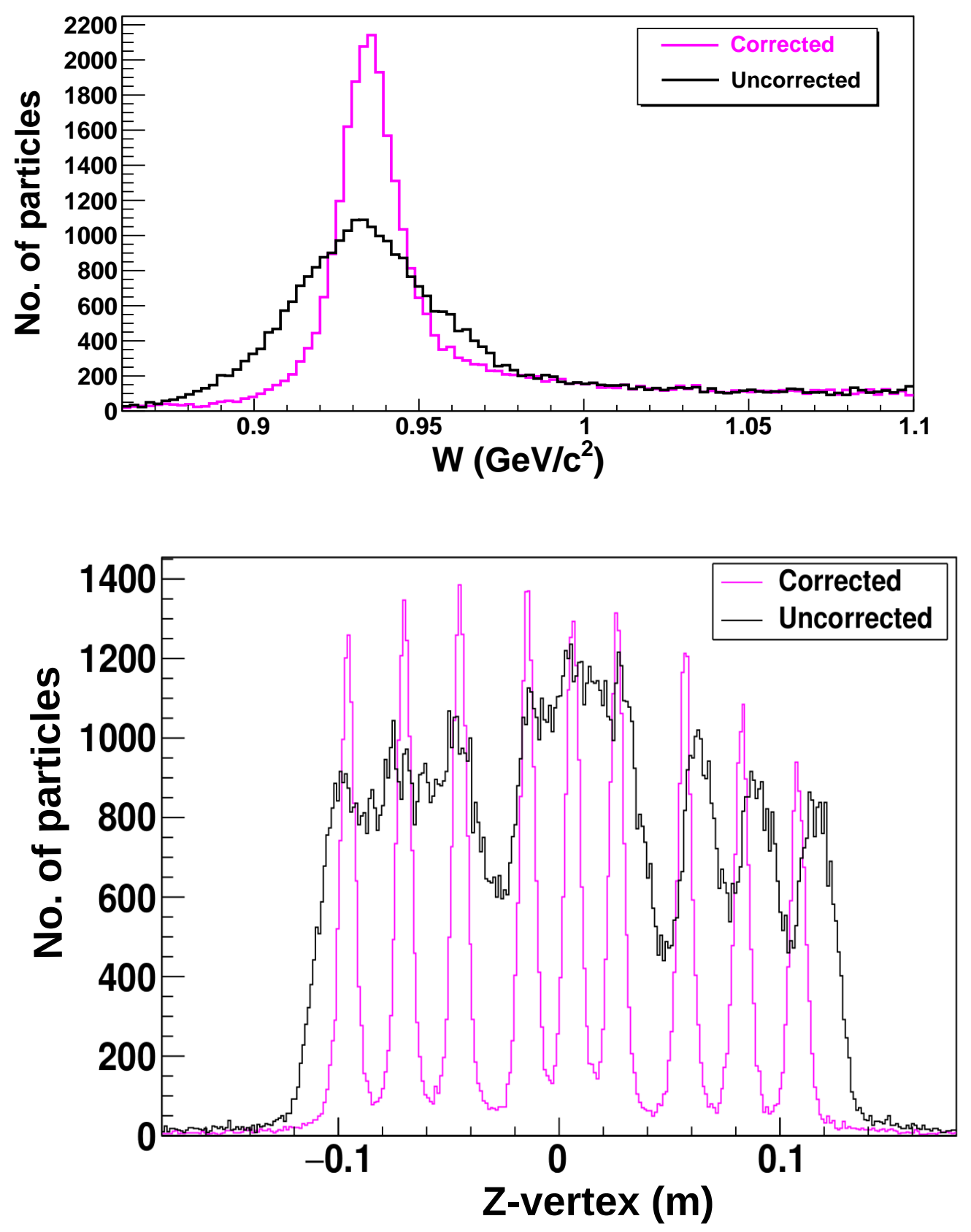

Figure 3.42. Reconstructed W and z-vertex resolution comparisons for nominal and saturation corrected optics.

on the lower side of the peak but the data resolution became much better. The $y_{\text {tar }}^{\prime} \mathrm{MC}$ width was not good at nominal optics but it also got improved significantly with corrected 

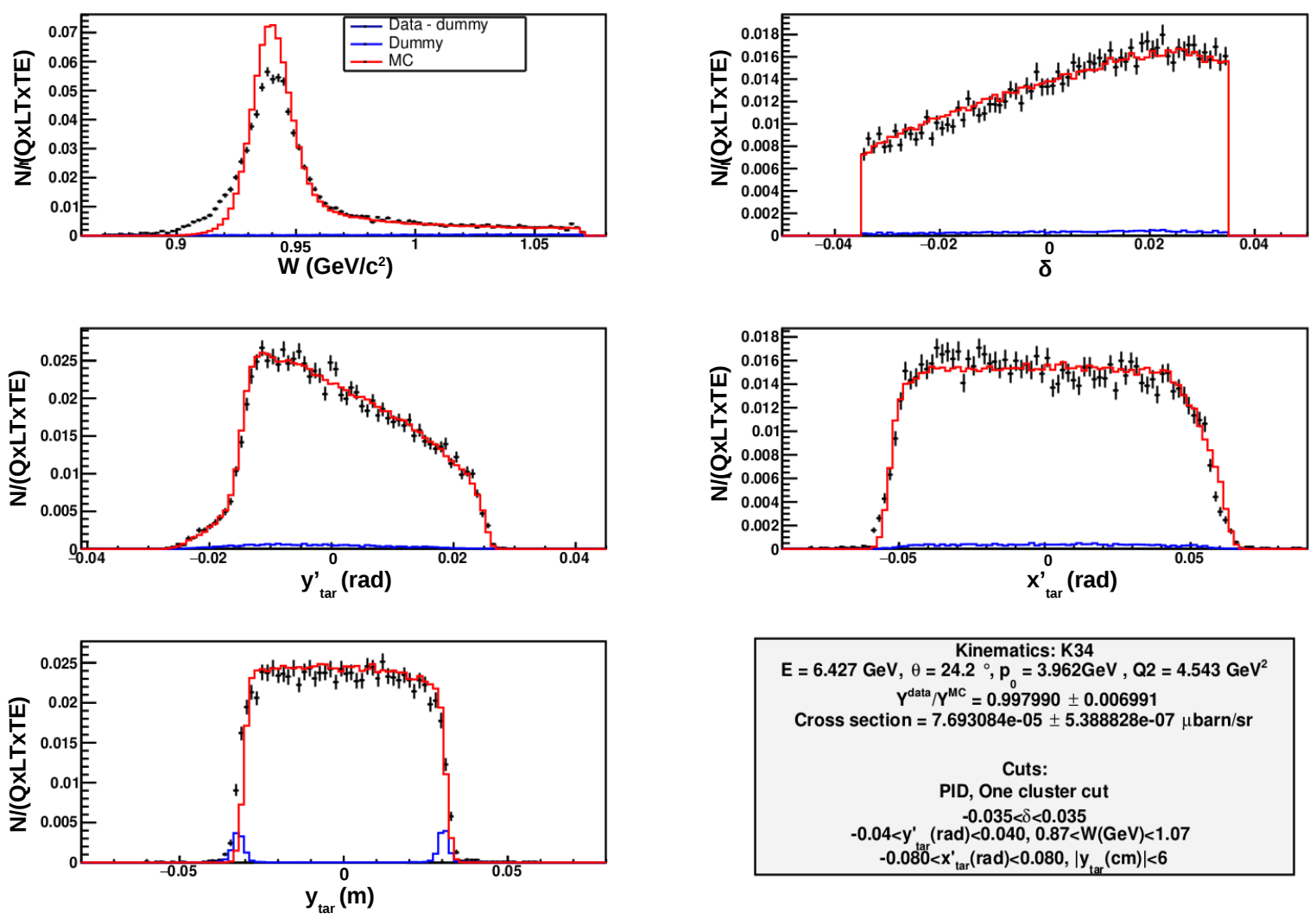

Figure 3.43. Data vs MC comparison for estimated Q1 $\int$ Bdl tune of 0.9315 Tm (baseline tune).

optics. Also there is a significant improvement in the other two target variables $x_{t a r}^{\prime}$ and $y_{\text {tar. }}$. The data to MC integral ratio has been improved by almost $2 \%$. In order to improve the $\mathrm{W}$ resolution, field was re-tuned in MC by changing the baseline field by about $0.75 \%$. The new data vs MC comparisons of target variables is shown in Fig. 3.44, which shows a significantly improved resolution in $\mathrm{W}$ and $y_{\text {tar }}^{\prime}$ distributions for all saturated kinematics. Table 3.13 shows the yield comparisons for nominal, baseline and final tune, whereas Table 3.14 shows data to MC integral ratio comparisons for each of those three cases. The change in acceptance by less than $3 \%$ is due to the change in forward tune and the cut dependence of data yield. 

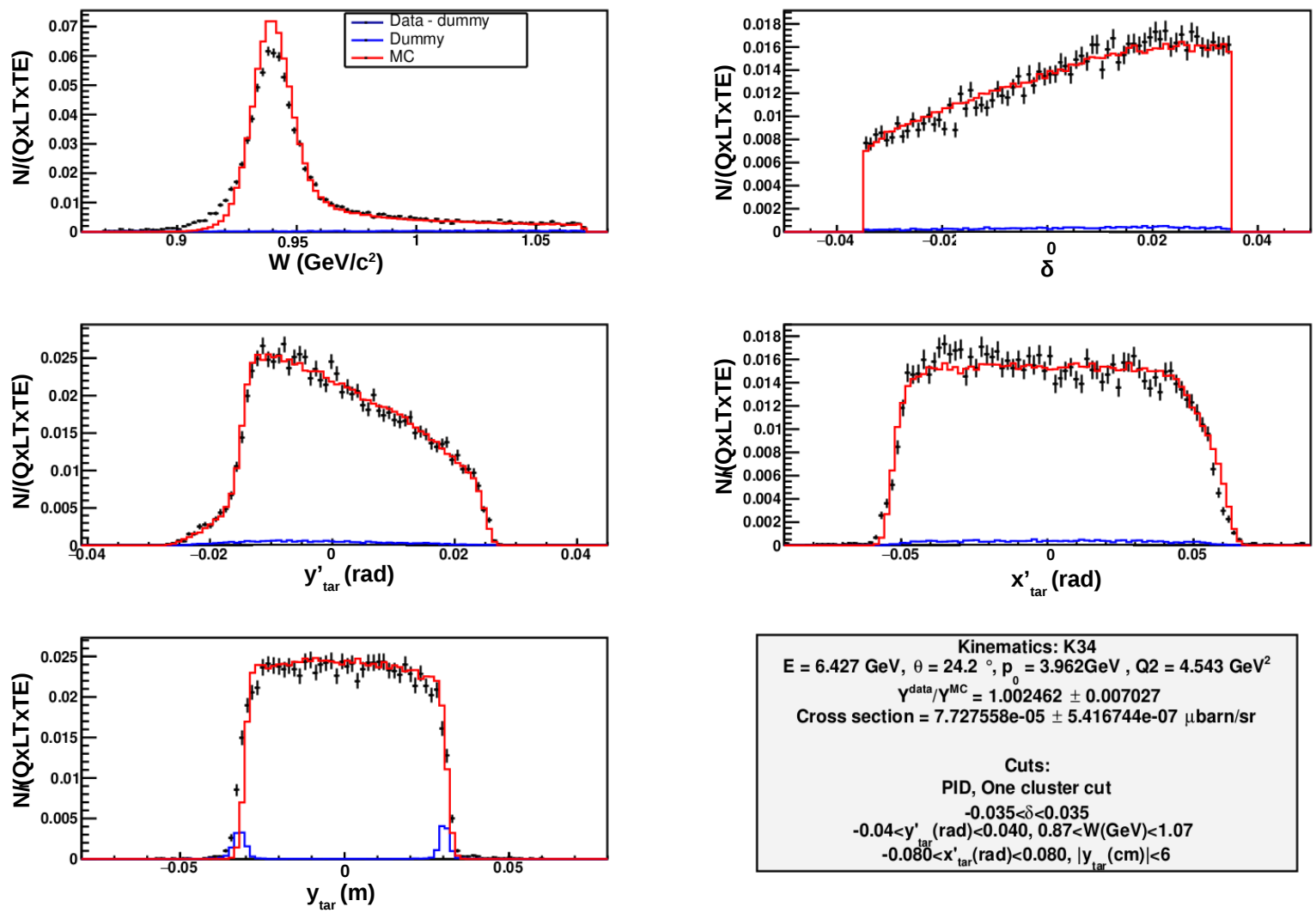

Figure 3.44. Data vs MC comparison for re-tuned $\int \mathrm{Bdl}$ of $0.925 \mathrm{Tm}$. 
Table 3.13

Integral comparisons for nominal, baseline and final tune.

\begin{tabular}{|l|l|l|l|l|l|l|}
\hline kinematic & $\begin{array}{l}\mathrm{Y}_{D} \\
(\text { Nomi- } \\
\text { nal })\end{array}$ & $\begin{array}{l}\mathrm{Y}_{M} \\
(\text { Nomi- } \\
\text { nal })\end{array}$ & $\begin{array}{l}\mathrm{Y}_{D} \\
\text { Base- } \\
\text { line })\end{array}$ & $\begin{array}{l}\mathrm{Y}_{M} \\
\text { (Base- } \\
\text { line) }\end{array}$ & $\begin{array}{l}\mathrm{Y}_{D} \text { (Fine } \\
\text { tune) }\end{array}$ & $\begin{array}{l}\mathrm{Y}_{M} \text { (Fine } \\
\text { tune) }\end{array}$ \\
\hline K3-4 & 0.936 & 0.961 & 0.921 & 0.924 & 0.919 & 0.918 \\
\hline K4-9 & 0.014 & 0.014 & 0.013 & 0.013 & 0.013 & 0.013 \\
\hline K3-6 & 0.134 & 0.139 & 0.134 & 0.134 & 0.133 & 0.134 \\
\hline K4-10 & 0.006 & 0.006 & 0.006 & 0.006 & 0.006 & 0.006 \\
\hline
\end{tabular}

Table 3.14

Data to $\mathrm{MC}$ slope ratio after using true $\int \mathrm{Bdl}$ in $\mathrm{MC}$.

\begin{tabular}{|l|l|l|l|l|l|l|l|}
\hline kinematic & $\mathrm{p} 0$ & $\theta$ & $\begin{array}{l}\text { Base line field } \\
\text { from } z \text { vs y } \\
\text { study }\end{array}$ & $\begin{array}{l}\text { Fine tune } \\
(\sim 0.75 \% \\
\text { change } \\
\text { from base- } \\
\text { line) }\end{array}$ & $\begin{array}{l}\mathrm{Y}_{\mathrm{D}} / \mathrm{Y}_{\mathrm{M}} \\
\text { (nomi- } \\
\text { nal) }\end{array}$ & $\begin{array}{l}\mathrm{Y}_{\mathrm{D}} / \mathrm{Y}_{\mathrm{M}} \\
\text { (base- } \\
\text { line) }\end{array}$ & $\begin{array}{l}\mathrm{Y}_{\mathrm{D}} / \mathrm{Y}_{\mathrm{M}} \\
\text { (Fine } \\
\text { tune) }\end{array}$ \\
\hline K3-4 & 3.962 & 24.2 & $0.9315 \pm 0.0005$ & 0.927 & 0.974 & 0.996 & 1.002 \\
\hline K4-9 & 3.685 & 30.9 & $0.9558 \pm 0.0004$ & 0.948 & 0.990 & 1.001 & 0.999 \\
\hline K3-6 & 3.224 & 30.9 & $0.9835 \pm 0.0006$ & 0.976 & 0.996 & 0.999 & 0.997 \\
\hline K4-10 & 3.259 & 34.4 & $0.9801 \pm 0.0002$ & 0.972 & 0.996 & 0.988 & 0.984 \\
\hline
\end{tabular}

\subsection{Uncertainties in Spectrometer Modeling for LHRS Fall 2016}

As determined by the MC the spectrometer acceptance is primarily determined by four of the 20 apertures for a delta cut of $\pm 3.5 \%$. The uncertainty on the real positions of the apertures is estimated to be around the mm level. Therefore, to study the corresponding uncertainty on the acceptance, each of these were moved by $1 \mathrm{~mm}$ up and down in the simulation using the uniform generator. To study the uncertainty in the solid angle due to the magnetic field integrals, the field of each quad was also changed in COSY. Table 3.15 shows the uncertainty due to the dominant aperture positions. 
Table 3.15

Study of MC uncertainties for kinematics K3-7.

\begin{tabular}{|c|c|c|}
\hline Aperture shift (in cm) & Solid angle $(\Omega)(\mathrm{msr})$ & $\Delta \Omega / \Omega_{0}(\%)$ \\
\hline No shift $(\mathrm{k} 3-7)$ & 5.989 & 0.00 \\
\hline $6(\mathrm{xs} 0.1)$ & 5.992 & 0.05 \\
\hline $6(\mathrm{xs}+0.1)$ & 5.998 & 0.15 \\
\hline $6(\mathrm{ys}-0.1)$ & 5.993 & 0.08 \\
\hline $6(\mathrm{ys}+0.1)$ & 5.998 & 0.15 \\
\hline $13(\mathrm{xs}-0.1)$ & 5.991 & 0.03 \\
\hline $13(\mathrm{xs}+0.1)$ & 5.978 & 0.18 \\
\hline $13(\mathrm{ys}-0.1)$ & 6.001 & 0.19 \\
\hline $13(\mathrm{ys}+0.1)$ & 5.985 & 0.06 \\
\hline $15(\mathrm{xs}-0.1)$ & 5.993 & 0.07 \\
\hline $15(\mathrm{xs}+0.1)$ & 5.99 & 0.02 \\
\hline $15(\mathrm{ys}-0.1)$ & 6.001 & 0.19 \\
\hline $15(\mathrm{ys}+0.1)$ & 5.995 & 0.10 \\
\hline $17(\mathrm{xs}-0.1)$ & 5.994 & 0.09 \\
\hline $17(\mathrm{xs}+0.1)$ & 5.997 & 0.13 \\
\hline $17(\mathrm{ys}-0.1)$ & 6.021 & 0.53 \\
\hline $17(\mathrm{ys}+0.1)$ & 6.002 & 0.32 \\
\hline
\end{tabular}

Absolute uncertainties are converted into percentage, and the average over up and down shifts was determined, and finally added in quadrature to get the total uncertainty of $\sim 0.52 \%$. Table 3.16 shows the uncertainties in the solid angle due to the change in quad field. The total uncertainty is about $0.45 \%$ for the estimated $\int \mathrm{Bdl}$ uncertainty of $0.25 \%$.

\subsection{Acceptance Studies: Separating Optics from Acceptance}

The effect of acceptance cuts across the position of the sieve slits where the optics are well constrained has been studied. Software cuts at the sieve slit position were utilized to determine the variation in extracted cross section. These cuts were placed through the 
Table 3.16

Study of MC uncertainties by shifting the dominant apertures up and down by $1 \mathrm{~mm}$.

\begin{tabular}{|c|c|c|c|}
\hline Quad & Solid angle $(\Omega)$ & $\Delta \Omega / \Omega_{0}(\%)(\mathrm{Bdl}$ of $1 \%)$ & $\Delta \Omega / \Omega_{0}(\%)(\mathrm{Bdl}$ of $0.25 \%)$ \\
\hline Default $(\mathrm{k} 3-7)$ & 5.989 & 0. & 0 \\
\hline Q1 field ${ }^{*} 1.01$ & 5.987 & 0.02 & 0.005 \\
\hline Q1 field ${ }^{*} 0.99$ & 6.040 & 1.00 & 0.25 \\
\hline Q2 field *1.01 & 6.123 & 2.24 & 0.5 \\
\hline Q2 field *0.99 & 5.929 & 1.00 & 0.25 \\
\hline Q3 field *1.01 & 5.981 & 0.13 & 0.03 \\
\hline Q3 field *0.99 & 6.043 & 0.90 & 0.22 \\
\hline Q2 field *1.005 & 6.070 & 1.36 & 0.34 \\
\hline Q2 field *0.995 & 5.990 & 0.03 & 0.008 \\
\hline
\end{tabular}

center of sieve slit hole positions, where optics is best constrained as illustrated in Fig. 3.45. Table 3.17 shows the ratio of cross sections with and without the cut for four different

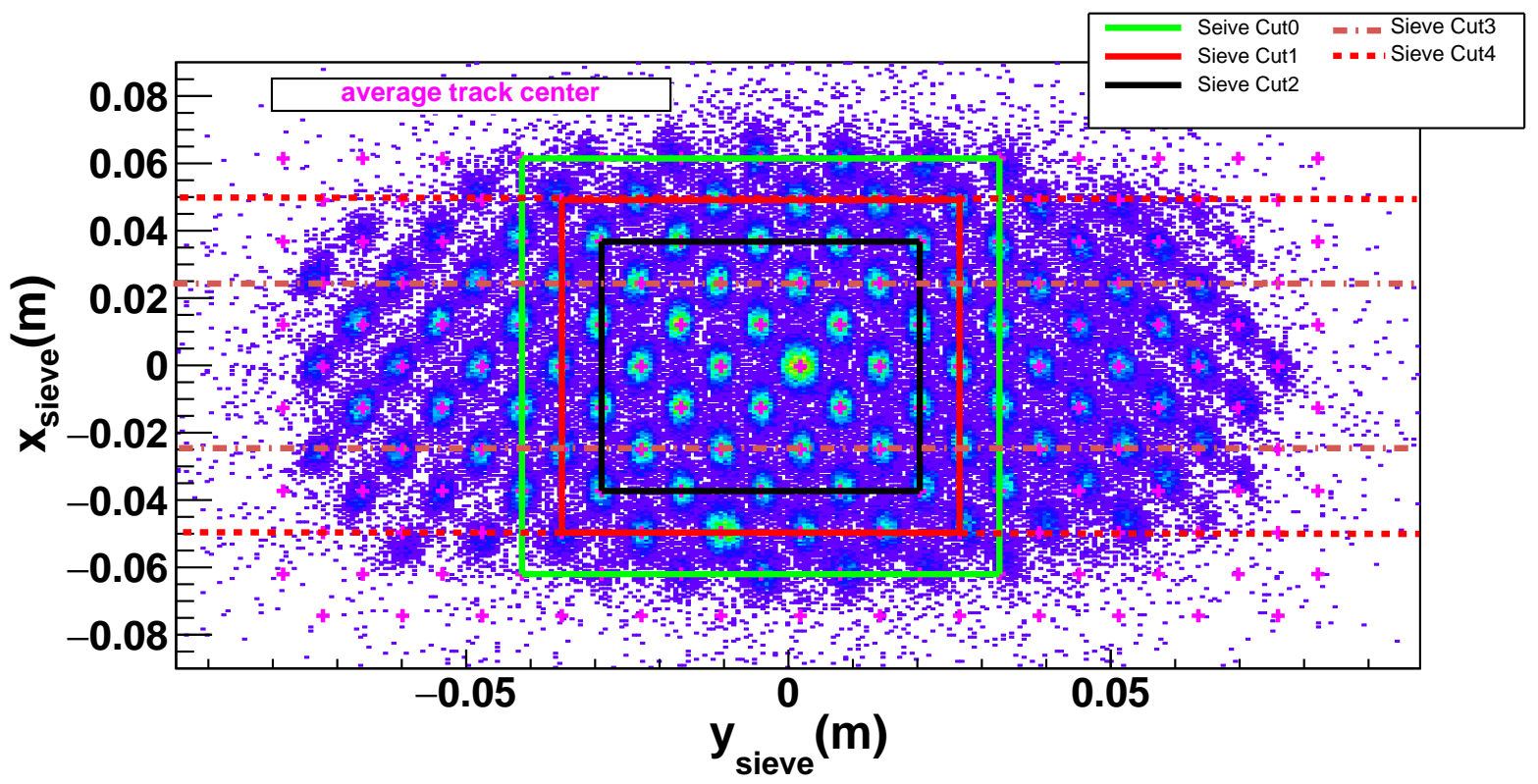

Figure 3.45. Software cuts on sieve collimater used to study spectrometer acceptance.

software cuts. The largest variation can be seen for the tightest cut. So, on average a 
variation in cross section of about $0.5 \%$ is assigned for the cuts that are least sensitive to optics.

Table 3.17

Ratio of cross sections with and without software cuts on the sieve collimater.

\begin{tabular}{|c|c|}
\hline cut & $\mathrm{d} \sigma / d \sigma_{\text {nocut }}$ \\
\hline No cut & 1.00 \\
\hline 0 & 0.987 \\
\hline 1 & 0.972 \\
\hline 2 & 1.002 \\
\hline 3 & 1.010 \\
\hline 4 & 0.999 \\
\hline
\end{tabular}




\section{CHAPTER 4 EXTRACTION OF THE ELASTIC CROSS SECTION}

The differential scattering cross section describes the probability of an incoming electron with energy $\mathrm{E}$ to interact with the target and to scatter at an angle $\theta$ with an scattered energy E' into the solid angle $\Delta \Omega$. The measured inclusive electron-proton cross section is given by

$$
\frac{d \sigma}{d \Omega}=\frac{R C}{\mathcal{L} \times \epsilon \times L T} \int d E^{\prime} \frac{N_{e^{-}\left(E^{\prime}, \theta\right)}-N_{B G}\left(E^{\prime}, \theta\right)}{A\left(E^{\prime}, \theta\right)} .
$$

Where

- $N_{e-}$ is the number of scattered electrons detected in each E' and $\theta$ beans,

- $N_{B G}$ represents the corresponding events from background processes,

- $\mathcal{L}$ is the integrated luminosity,

- $\epsilon$ is the efficiencies product of the trigger, particle identification and tracking,

- LT is the computer live time,

- $\mathrm{RC}$ is the radiative correction (section 3.11),

- $A\left(E^{\prime}, \theta\right)$ fractional acceptance of the spectrometer,

- and $\theta$ is the scattering angle.

The integrated luminosity can be calculated from

$$
\mathcal{L}=\frac{n_{e} n_{p}}{a}=\frac{Q}{e} \rho l \frac{Z}{A} N_{A},
$$


where, $n_{e}$ and $n_{p}$ are the number of electron beam and number of protons respectively, $a$ is the target area, $l$ is the length of the target, $\mathrm{A}$ is atomic mass of the target and $N_{A}$ is the Avogadro's number. Two methods were used to extract differential scattering cross section to take into account the acceptance:

- The Monte Carlo ratio method

- The acceptance correction method

The main method was the first method and the second, complementary method was utilized to check the cross section extracted from the first method as this method is sensitive to different systematic uncertainties than the first method. The second method will be discussed in section 4.0.1.4.

\subsubsection{The Monte Carlo Ratio Method}

The relationship between the model cross section and simulated yield can be written similar to equation 4.1 for the data as

$$
\left(\frac{d \sigma}{d \Omega}\right)_{m o d}=\frac{R C^{M C}}{L_{M C}} \int d E^{\prime} \frac{N_{e-}^{M C}}{A^{M C}\left(E^{\prime}, \theta\right)} .
$$

For the same normalized luminosity the ratio of equations 4.1 and 4.3 yields

$$
\left(\frac{d \sigma}{d \Omega}\right)_{d a t a} /\left(\frac{d \sigma}{d \Omega}\right)_{\bmod }=\frac{R C^{d a t a}}{R C^{M C}} \frac{\int d E^{\prime}\left(N_{e-}^{d a t a}-N_{B G}\right)}{\int d E N_{e-}^{M C}} \cdot \frac{A^{M C}\left(E^{\prime}, \theta\right)}{A^{\text {data }}\left(E^{\prime}, \theta\right)}
$$

Assuming that the acceptance and radiative contributions are correctly modeled, the data cross section can be determined from

$$
\left(\frac{d \sigma}{d \Omega}\right)_{d a t a}=\left(\frac{d \sigma}{d \Omega}\right)_{m o d} \cdot \frac{Y^{d a t a}}{Y_{M C}} .
$$




$$
\left(\frac{d \sigma}{d \Omega}\right)_{M C}=\frac{\sigma_{M o t t}}{1+\tau}\left[G_{E}^{2}+\frac{\tau}{\epsilon} G_{M}^{2}\right]
$$

where, $\tau=Q^{2} / 2 M^{2}$. The input model cross section to the simulation is discussed in section 1.6, and is taken from a fit to the existing cross section data in single photon approximation. So, the data cross section, using parametrized model form factors is given by

$$
\left(\frac{d \sigma}{d \Omega}\right)_{d a t a}=\left(\frac{Y^{\text {data }}}{Y_{M C}}\right) \cdot \frac{\sigma_{M o t t}}{1+\tau}\left[G_{E}^{2}+\frac{\tau}{\epsilon} G_{M}^{2}\right] .
$$

\subsubsection{Selection of Good Electron Events}

In this section, the data selection cuts applied to both data and simulated events to select elastically scattered electrons is discussed. The cuts applied to the data sample include

Table 4.1

Cuts applied to select good electron events.

\begin{tabular}{|c|}
\hline Acceptance cuts \\
\hline \hline$-40<\mathrm{y}_{\text {tar }}^{\prime}<40(\mathrm{mrad})$ \\
\hline$-80<\mathrm{x}_{\text {tar }}<80(\mathrm{mrad})$ \\
\hline$-3.5 \%<\delta<3.5 \%$ \\
\hline $0.86<W<1.05\left(\mathrm{GeV} / \mathrm{c}^{2}\right)$ \\
\hline \hline PID Cuts \\
\hline \hline No. of photo-electrons $>4$ \\
\hline $\mathrm{E}_{\text {cal }} / \mathrm{p}>0.6$ \\
\hline \hline Single cluster cuts \\
\hline Trigger 1 events \\
\hline
\end{tabular}

PID, single cluster, and acceptance cuts and those applied to which are given in Table 4.1 whereas those for simulated events are the corresponding acceptance cuts. 


\subsubsection{Subtraction of Background Events from Target Cell Endcaps}

The target cell containing liquid hydrogen has aluminum windows which can be a source of background. During data taking on the LH2 target, some of the incoming electrons scattered from the aluminum windows of the target cell are also detected in the spectrometer. These background events have to be subtracted from the yield measured in order to estimate the yield for scattering from the LH2 target only. In order to determine the amount of this background, data on the aluminum dummy target were taken in the same kinematics as the LH2 data. After measuring the dummy yield, the corrected LH2 yield can be determined from:

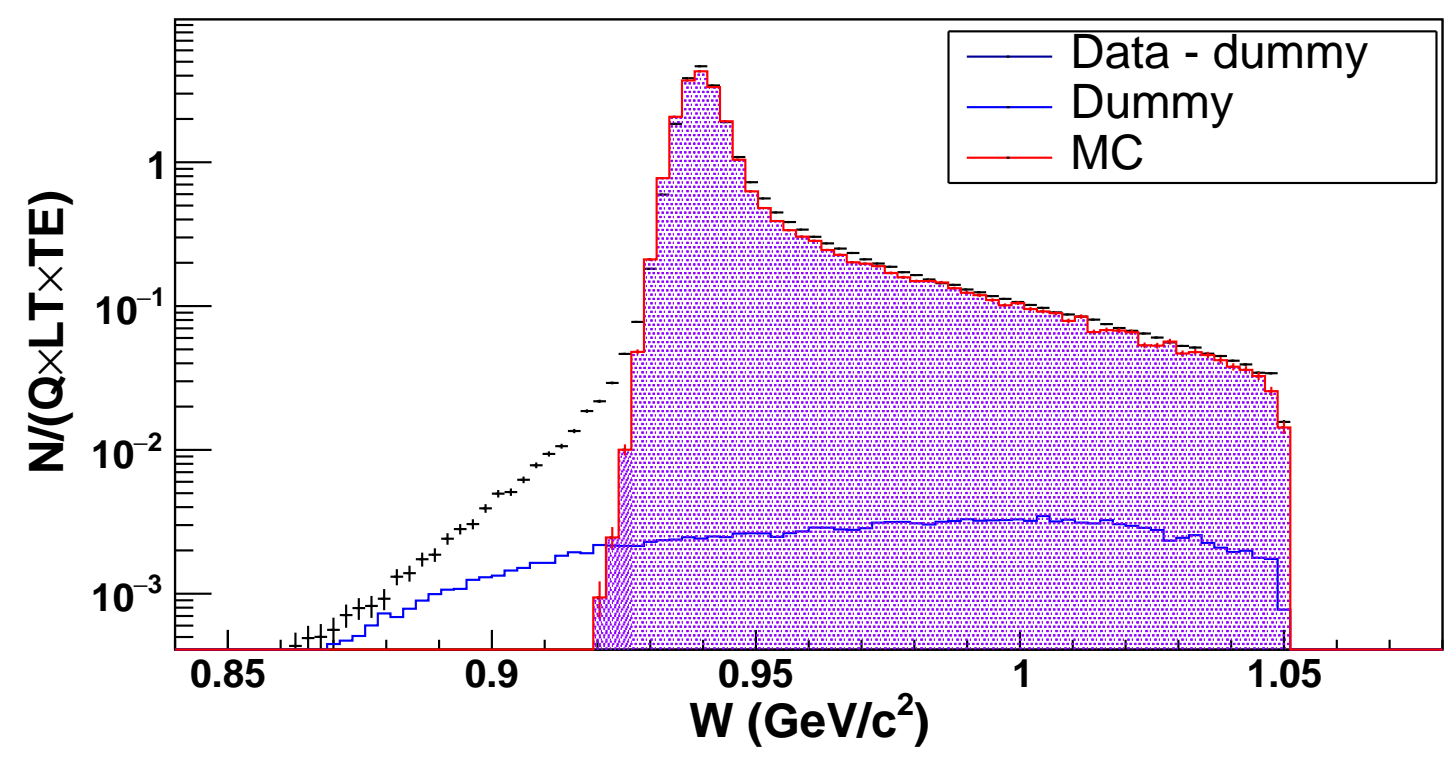

Figure 4.1. Normalized yield w distribution for LH2 target. The red solid line is the MC normalized yield, black points are the background subtracted data, and the blue solid line is the events from Aluminum windows of the LH2 target.

$$
Y_{\text {corrected }}=Y_{L H 2}-Y_{\text {dummy }} \cdot \frac{T_{\text {wall }}}{T_{\text {dummy }}} * C_{b p}
$$

where, the $Y$ are the yields for LH2 and dummy targets, $T_{\text {wall }}$ and $T_{\text {dummy }}$ are the target wall and dummy thickness, respectively. The factor $C_{b r}$ represents the ratio of radiative 
correction due to the difference in target thicknesses found to be 1.022 for all kinematics from the L. Ou study [59]. For Fall 2016 run, the ratio of wall to dummy thickness is given by:

$$
\begin{aligned}
\frac{T_{\text {wall }}}{T_{\text {dummy }}} & =\frac{T_{\text {entrance }}+T_{\text {exit }}}{T_{\text {dummy }}} \\
& =\frac{(0.0175+0.011) \times 0.2815}{0.48} \\
& =0.169
\end{aligned}
$$

The uncertainty in cross section due to the aluminum background is mainly coming from the thickness of the cell tip of $\pm 10 \%$ and that of cell entrance of $\pm 5 \%$. As the quasi-elastic electrons account for about $3 \%$ of all events, contribution to the uncertainty in the final yield from background for all kinematics is about $0.2 \%$ from the study of L. Ou [59]. Since the quasi-elastic cross section model does not work for the kinematics K5-16 due to the very large $Q^{2}, 1 \%$ uncertainty is assigned for this kinematics.

\subsubsection{Cross Section Extraction}

Fig. 4.2 shows the data vs MC comparison of five target variables: invariant mass of proton $(W)$, relative momentum $(\delta)$, in-plane angle $\left(y_{t a r}^{\prime}\right)$, out of plane angle $\left(x_{t a r}^{\prime}\right)$ and target $\mathrm{y}\left(y_{t a r}\right)$ for the validation kinematics K1-1 where the cross section is known well. The first panel shows kinematic information, extracted cross section, and cuts applied. In order to obtain the differential scattering cross section in this method, all of the target variables between the data and MC should match well. Looking at the shapes of $\delta, y_{\text {tar }}^{\prime}$ and $y_{\text {tar }}$ it is clear that the MC model is not perfectly matching with reality. In addition, the extracted cross section is found to disagree with the model cross section, and the data integral is found to be about $4.4 \%$ higher than the MC integral. All magnets and apertures that are sensitive to the change have been checked. Finally, it was concluded that the quadrupole Q2 was off by 

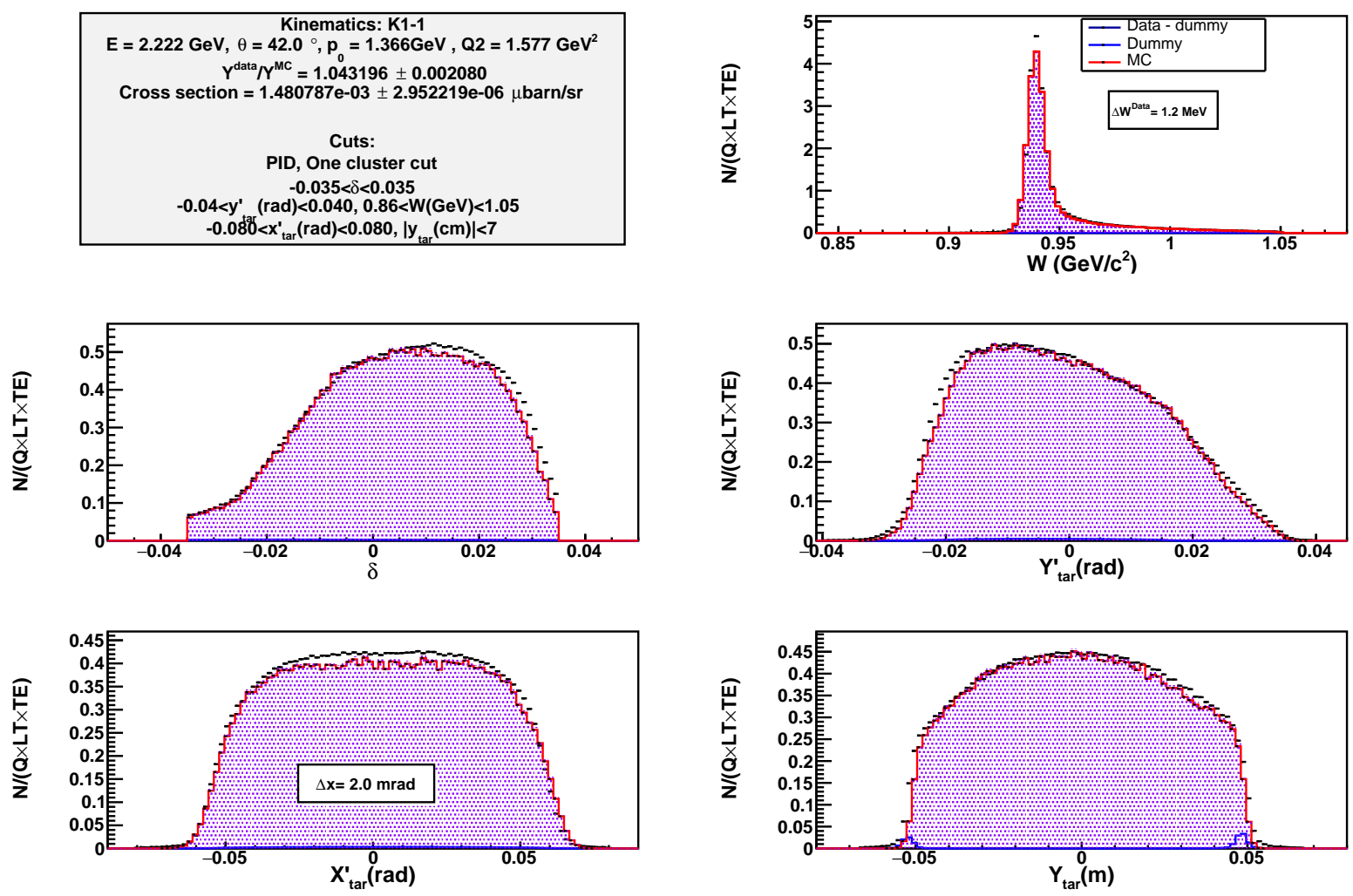

Figure 4.2. Data yield vs MC yield comparison of kinematics K1-1. The red line shows the MC normalized yield, black points are the background subtracted data, and the blue line shows the events from aluminum windows of the LH2 target.

about $0.9 \%$. This adjustment improved the shape of the target distribution for the validation kinematics. In addition to this, the extracted cross section became closer to the well-known cross-section for this kinematics as shown in figure 4.3. This adjustment made less impact on the high $\mathrm{Q}^{2}$ kinematics. Fig. 4.4 shows the data vs MC comparison for kinematics with $\mathrm{Q}^{2}=4.5 \mathrm{GeV}^{2}$. This is the kinematics with Q1 saturation with a tuned Q1 field. Also, the Q2 field was increased by $0.9 \%$ for this kinematics but found very little impact. Fig. 4.5 and 4.6 shows the data vs MC comparison for kinematics with $\mathrm{Q}^{2}=8 \mathrm{GeV}^{2}$ and $\mathrm{Q}^{2}=11 \mathrm{GeV}^{2}$ respectively. The spectrometer pointing offsets are included in all kinematics. In addition to this, a shift of about $-0.3 \mathrm{~mm}$ in the target ladder was included in the analysis. 

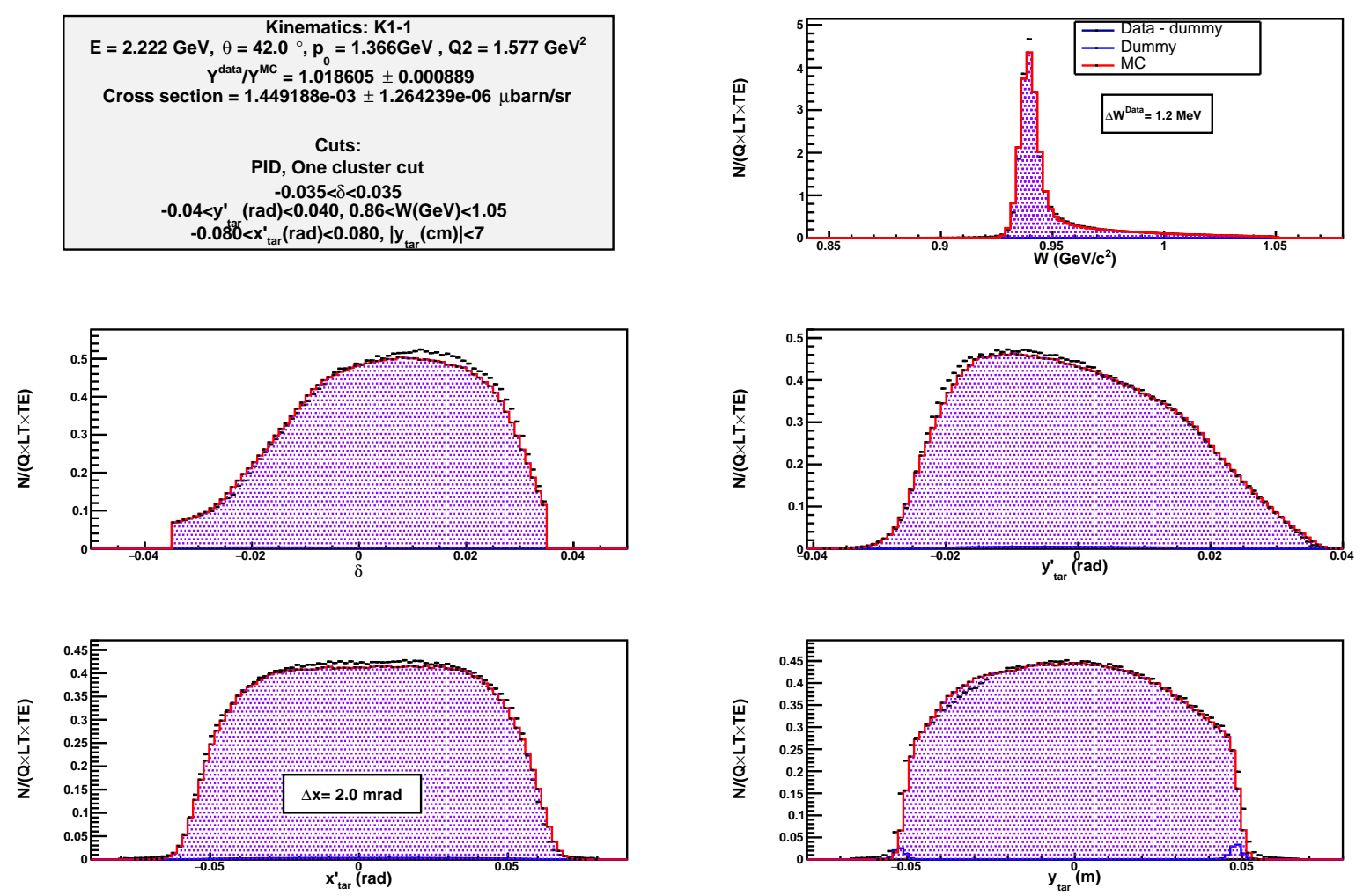

Figure 4.3. Data yield vs MC yield comparison of the validation kinematics K1-1 after increasing field of Q2 by $0.9 \%$. The red line shows the MC normalized yield, black points are the background subtracted data, and the blue line shows the events from aluminum windows of the LH2 target.

\subsubsection{Systematic Uncertainties}

The total systematic uncertainty in the cross section is taken as the sum in quadrature of all systematic uncertainties on the quantities that contribute to the cross section. There are two types of systematic uncertainties:

- Point-to-point uncertainties: This type of uncertainties changes between each run or each kinematic. These types of uncertainties are caused by changes in experimental conditions during data taking, and therefore their effect is uncorrelated between different data points. These include uncertainties arising from a change in the detector 

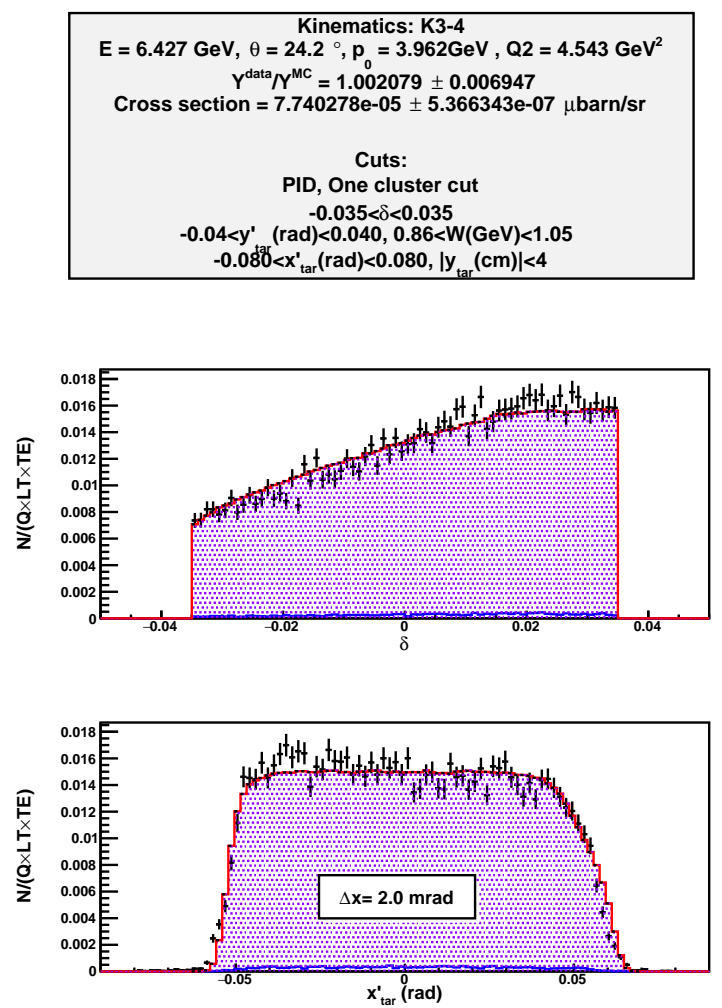
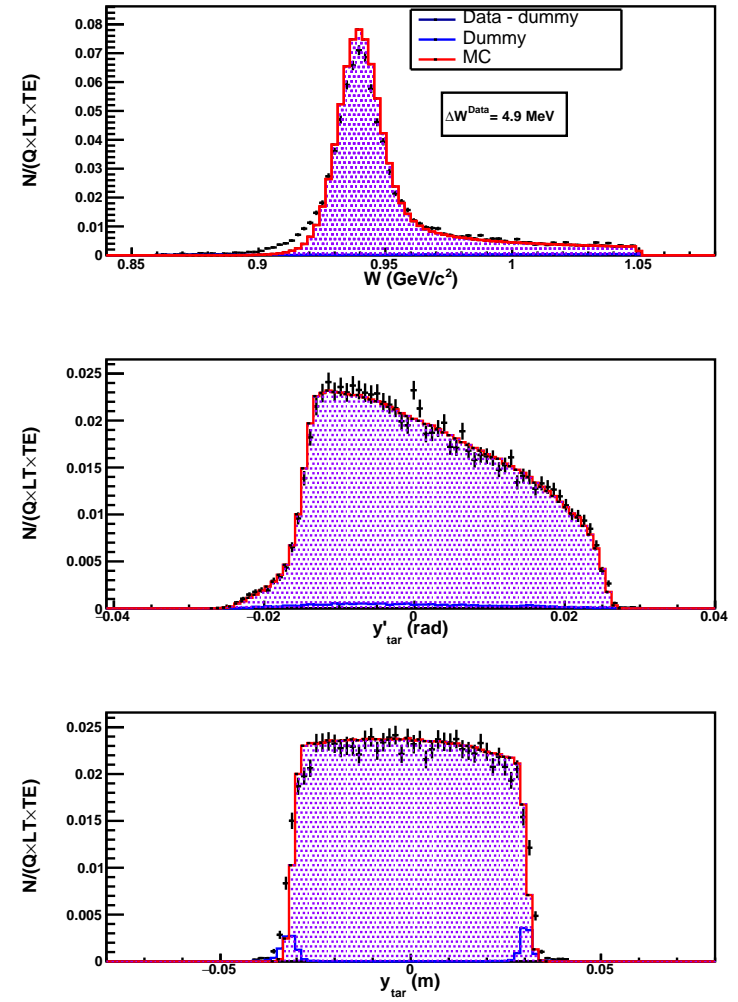

Figure 4.4. Data yield vs MC yield of kinematics K3-4 after increasing the field of Q2 by $0.9 \%$. The red line shows the MC normalized yield, black points are the background subtracted data, and the blue line shows the events from aluminum windows of the LH2 target.

efficiencies and data acquisition systems, and changes from one spectrometer setting to another.

- Normalization uncertainties: This type of uncertainties affect all kinematics similarly. An example of these uncertainty can be a systematic shift in the offset in the beam current measurement device, the acceptance, the target thickness measurement, etc.

These uncertainties are presented in the Tab. 4.2. The kinematics taken in parallel with the DVCS experiment were with low beam current and their uncertainties in the beam current are larger than the GMp data taken in a dedicated run period. The uncertainties on the radiative correction is mainly due to the choice of cutoff in $\mathrm{W}$ spectra. The typical point-to- 

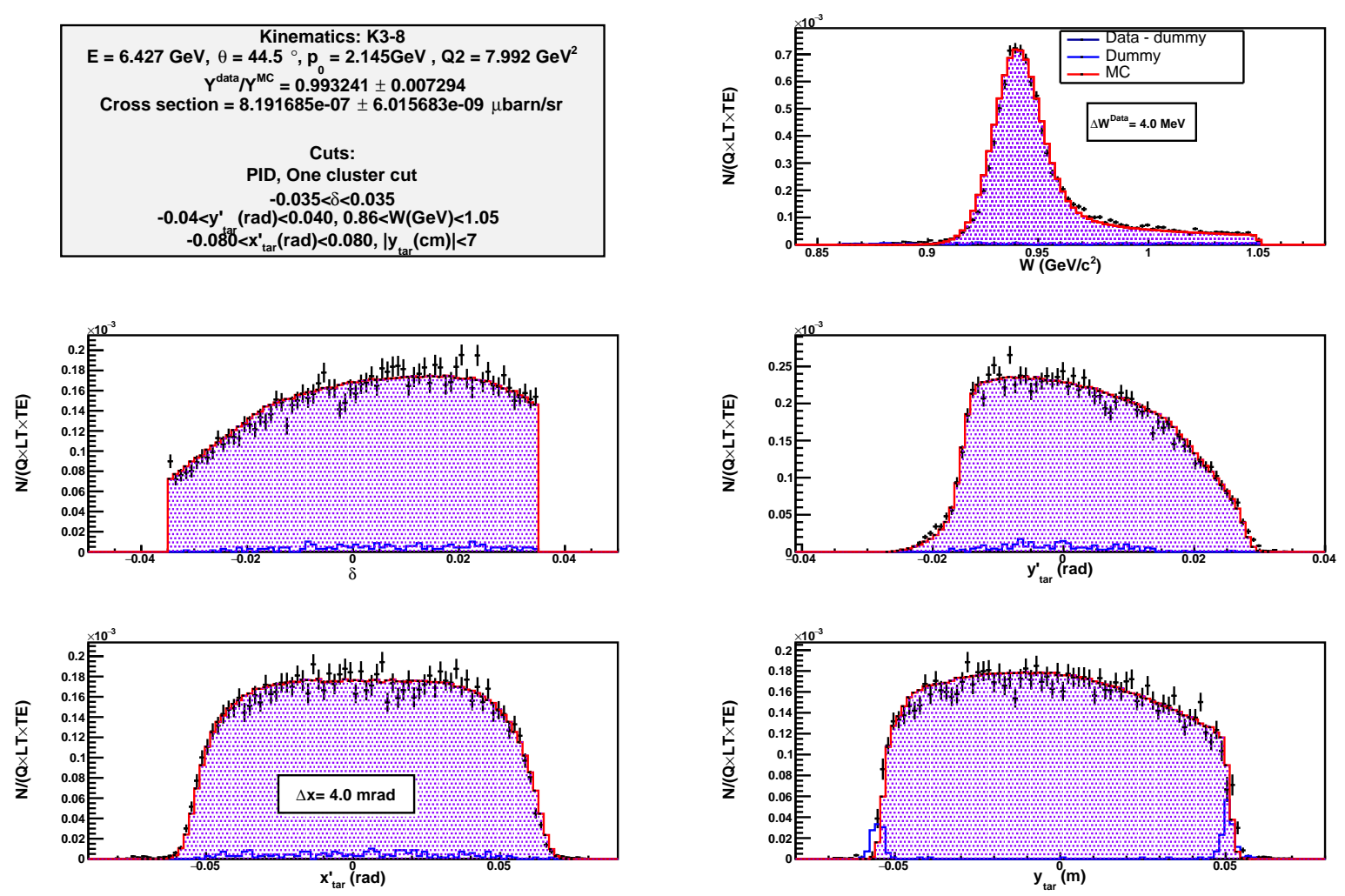

Figure 4.5. Data yield vs MC yield comparison of kinematics K3-8 after increasing field of Q2 by $0.9 \%$. The red line shows the MC normalized yield, black points are the background subtracted data, and the blue line shows the events from aluminum windows of the LH2 target.

point uncertainty for the $\mathrm{W}$ cutoff ranging from $1 \mathrm{GeV} / \mathrm{c}^{2}$ to $1.06 \mathrm{GeV} / \mathrm{c}^{2}$ is about $0.8 \%$. In addition to this the normalization between the largest and smallest values of the $\mathrm{W}$ cutoff was found to be about $1.0 \%$. The difference is mainly coming from the shapes of the radiative tail in the data and simulation as well as the result of unoptimized resolution matching of the drift chamber and the optics matrices [8]. The model dependence of the cross section was observed by calculating cross section using different models which is about $0.1 \%$ overall. The rest of the uncertainties are discussed in respective chapters. The summary of the GMp Fall 2016 LHRS kinematics, extracted elastic cross sections, point-to-point and statistical uncertainties are presented in Tab. 4.4. 
Table 4.2

Summary of point-to-point and normalization uncertainties of the Fall 2016 LHRS GMp experiment.

\begin{tabular}{|c|c|c|}
\hline Source & $\mathrm{d} \sigma / \sigma(\%)(\mathrm{pt}-\mathrm{pt})$ & $\mathrm{d} \sigma / \sigma(\%)$ (Norm.) \\
\hline Beam charge $(\Delta I=0.06 \mu \mathrm{A})$ & $0.6($ at $10 \mu \mathrm{A})-0.1($ at $65 \mu \mathrm{A})$ & 0.1 \\
\hline Scattering angle $(\Delta \theta=0.2 \mathrm{mrad})$ & $0.1-0.4$ & $0.1-0.4$ \\
\hline Beam energy $\left(\Delta E=5 \times 10^{-4}\right)$ & 0.3 & 0.3 \\
\hline Boiling & $<0.35($ at $10 \mu \mathrm{A})-0($ at $60 \mu \mathrm{A})$ & $0.35($ at $60 \mu \mathrm{A})$ \\
\hline Optics & 0.3 & 0.3 \\
\hline Track Reco & 0.2 & 0.2 \\
\hline PID & 0.1 & 0.1 \\
\hline Trigger & 0.2 & 0.1 \\
\hline Target Length & 0.7 & 0.1 \\
\hline Spectrometer acceptance & 0.8 & 0.8 \\
\hline Radiative correction & 0.2 & 1.0 \\
\hline Background subtraction & & 0.2 \\
\hline Cross section model & $1.2-1.3 \%$ & 0.1 \\
\hline Total & & $1.4-1.6 \%$ \\
\hline
\end{tabular}

Table 4.3

Summary of the GMp Fall 2016 LHRS kinematics, extracted elastic cross sections, point-topoint and statistical uncertainties.

\begin{tabular}{|l|l|l|l|l|l|l|l|l|}
\hline Kinematic & $\begin{array}{l}E_{0} \\
(\mathrm{GeV})\end{array}$ & $\begin{array}{l}Q^{2} \\
\left(\mathrm{GeV}^{2}\right)\end{array}$ & $\begin{array}{l}\text { Ave. cur- } \\
\text { rent }(\mu \mathrm{A})\end{array}$ & $\begin{array}{l}\mathrm{d} \sigma / d \Omega \\
(\mu b / s r)\end{array}$ & $\begin{array}{l}\mathrm{d} \sigma / \sigma \\
(\mathrm{Pt}-\mathrm{pt}) \\
(\%)\end{array}$ & $\begin{array}{l}\mathrm{d} \sigma / \sigma \\
(\text { Stat. }) \\
(\%)\end{array}$ & $\begin{array}{l}\mathrm{d} \sigma / \sigma \\
(\text { Norm. }) \\
(\%)\end{array}$ & $\begin{array}{l}\text { Pt-pt } \\
+ \text { Stat. } \\
(\%)\end{array}$ \\
\hline $\mathrm{k} 1-1$ & 2.222 & 1.577 & 56.831 & $1.449 \mathrm{E}-03$ & 1.2 & 0.1 & 1.4 & 1.2 \\
\hline $\mathrm{K} 3-4$ & 6.427 & 4.543 & 64.071 & $7.740 \mathrm{E}-05$ & 1.3 & 0.7 & 1.6 & 1.5 \\
\hline $\mathrm{K} 3-6$ & 6.427 & 5.947 & 66.521 & $1.108 \mathrm{E}-05$ & 1.3 & 0.6 & 1.5 & 1.4 \\
\hline $\mathrm{K} 3-7$ & 6.427 & 6.992 & 64.605 & $2.898 \mathrm{E}-06$ & 1.2 & 0.7 & 1.5 & 1.4 \\
\hline $\mathrm{K} 3-8$ & 6.427 & 7.992 & 65.067 & $8.192 \mathrm{E}-05$ & 1.3 & 0.7 & 1.4 & 1.5 \\
\hline $\mathrm{K} 4-9$ & 8.518 & 9.002 & 67.155 & $1.285 \mathrm{E}-06$ & 1.3 & 0.7 & 1.5 & 1.5 \\
\hline $\mathrm{K} 4-10$ & 8.518 & 9.807 & 66.345 & $5.871 \mathrm{E}-07$ & 1.3 & 0.7 & 1.5 & 1.5 \\
\hline $\mathrm{K} 4-11$ & 8.518 & 11.18 & 65.434 & $1.543 \mathrm{E}-07$ & 1.2 & 1.0 & 1.4 & 1.6 \\
\hline
\end{tabular}



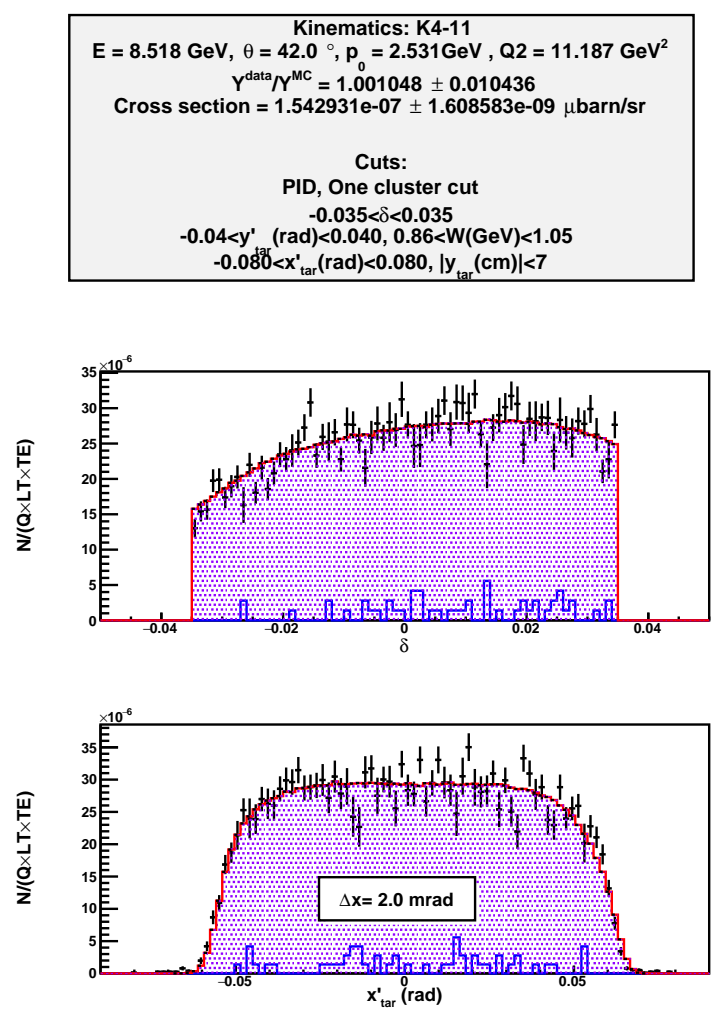
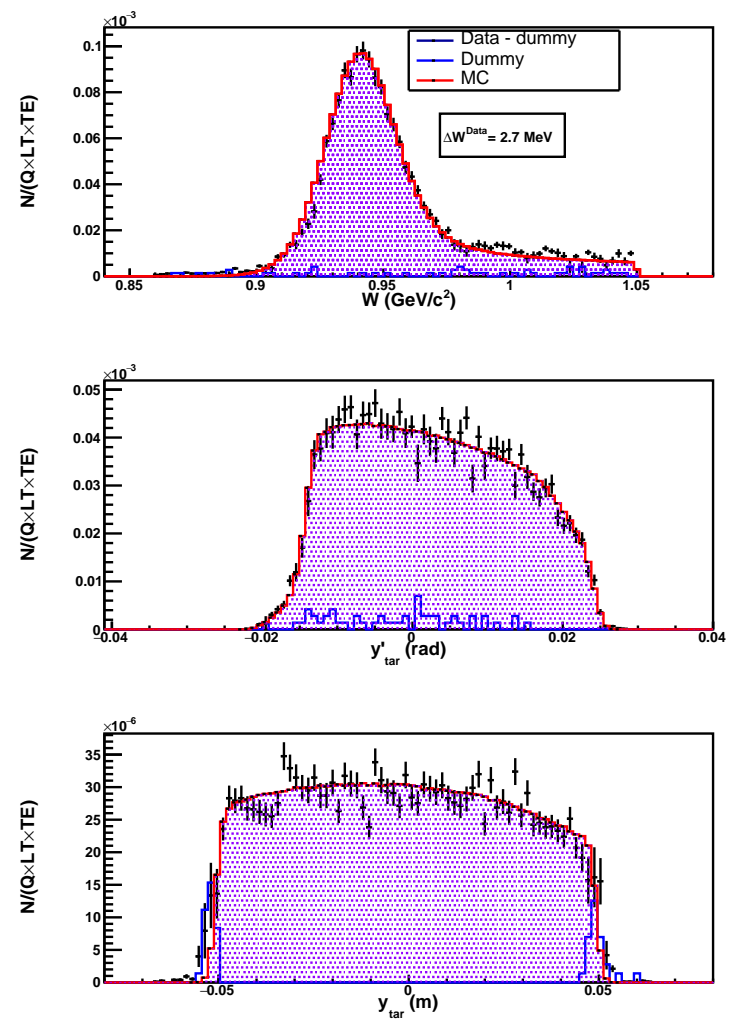

Figure 4.6. Data yield vs MC yield comparison of kinematics K4-11 after increasing field of Q2 by $0.9 \%$. The red line shows the $\mathrm{MC}$ normalized yield, black points are the background subtracted data, and the blue line shows the events from aluminum windows of the LH2 target. 
Table 4.4

Summary of the GMp Fall 2016 RHRS kinematics, extracted elastic cross sections, point-topoint and statistical uncertainties (uncertainties are not final).

\begin{tabular}{|l|l|l|l|l|l|l|l|}
\hline Kinematic & $\begin{array}{l}E_{0} \\
(\mathrm{GeV})\end{array}$ & $\begin{array}{l}Q^{2} \\
\left(\mathrm{GeV}^{2}\right)\end{array}$ & $\begin{array}{l}\mathrm{d} \sigma / d \Omega \\
(\mu b / s r)\end{array}$ & $\begin{array}{l}\mathrm{d} \sigma / \sigma \\
(\mathrm{Pt}-\mathrm{pt}) \\
(\%)\end{array}$ & $\begin{array}{l}\mathrm{d} \sigma / \sigma \\
(\text { Stat. }) \\
(\%)\end{array}$ & $\begin{array}{l}\mathrm{d} \sigma / \sigma \\
(\text { Norm. }) \\
(\%)\end{array}$ & $\begin{array}{l}\text { Pt-pt } \\
+ \text { Stat. } \\
(\%)\end{array}$ \\
\hline $\mathrm{k} 1-2$ & 2.222 & 1.858 & $5.299 \mathrm{E}-04$ & 1.8 & 0.2 & 2.6 & 1.8 \\
\hline K3-9 & 6.427 & 9.053 & $2.054 \mathrm{E}-07$ & 1.9 & 1.0 & 2.1 & 2.1 \\
\hline K4-13 & 8.518 & 12.568 & $3.552 \mathrm{E}-08$ & 2.1 & 2.3 & 2.5 & 3.1 \\
\hline K5-15 & 10.587 & 15.755 & $1.358 \mathrm{E}-08$ & 2.2 & 1.8 & 2.5 & 2.3 \\
\hline K4-12 & 8.518 & 12.069 & $6.135 \mathrm{E}-08$ & 1.8 & 1.2 & 2.2 & 2.2 \\
\hline
\end{tabular}

\subsubsection{Cross Section Extraction by Acceptance Correction Method}

The goal of this analysis is to extract the electron-proton differential cross section at fixed central angle. For this, data were binned in two dimensional $E^{\prime}$ and $\theta$ bins. In this analysis, binning in $E^{\prime}$ and $\theta$ are converted to a binning in $\delta$ and $\Delta \theta\left(=\theta-\theta_{0}\right)$ respectively, which are more suitable for application of the acceptance correction. For $\delta$, the binning chosen was 30 bins over the range of $\pm 6 \%$, whereas for the $\Delta \theta$ binning chosen was 90 bins over the range of $\pm 6 \mathrm{mrad}$.

The cross-section expressed in equation 4.1 does not only include single photon exchange but contains the higher order QED effects such as two-photon exchange, emission of bremsstrahlung photon before and after the scattering and virtual particle loops. The large radiative tail in the reconstructed invariant mass distribution is due to the emission of bremsstrahlung photons. In order to compare to the OPEA cross section, it requires that the tail be integrated into some cutoff in $E^{\prime}$, with a correction factor that includes higher order effects. The integration was cut off at $W_{\max } \leq 1.05 \mathrm{GeV} / \mathrm{c}^{2}$ to avoid the threshold for single photon production. The final extracted cross section corrected for detector efficiencies, effective solid angle, effective charge and counts from background events is then determined from the 
following equation,

$$
\frac{d \sigma(\theta)}{d \Omega}=\frac{R C\left(W_{\max }\right)}{\mathcal{L} \times \epsilon} \int^{W_{\max }} d E^{\prime} \frac{N\left(E^{\prime}, \theta\right)-N_{B G}\left(E^{\prime}, \theta\right)}{\Delta \Omega\left(E^{\prime}, \theta\right)}
$$

where $N_{B G}$ is the number of background events, $\mathcal{L}$ is the integrated luminosity, $\epsilon$ is the product of the detector efficiencies and $\Delta \Omega$ is the effective solid angle.
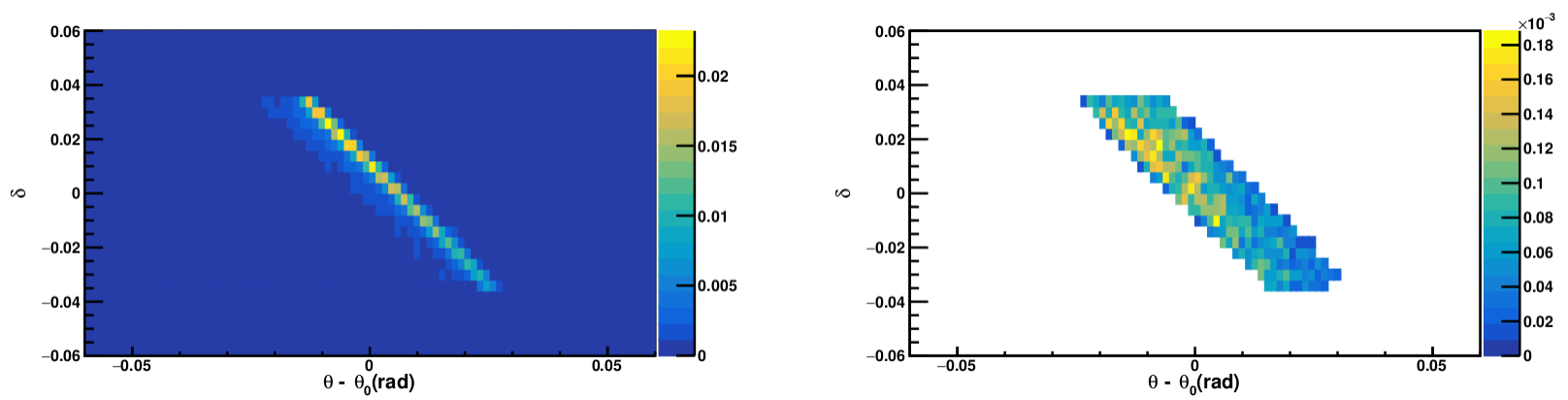

Figure 4.7. Left: Liquid hydrogen charge-normalized yield binned on 2-D grid in $\delta$ and $\theta$ bins Right: Dummy charge-normalized yield binned on 2-D grid in $\delta$ and $\theta$ bins.

\subsubsection{Procedure}

For the elastic scattering cross section calculation, the radiative correction should be applied for every $\theta$ bin after summing over the $\delta$ bins. So, the procedure to calculate the cross-section is:

- For each two dimensional bin, calculate the efficiency corrected and dummy subtracted yield after applying the cuts.

- Calculate the effective solid angle for each two-dimensional bin and divide the corrected yield by effective solid angle.

- Sum over the $\delta$ bins to get bins just in $\theta$.

- For each $\theta$ bin, run the standalone radiative correction code and calculate a correction factor. 
- Calculate the bin-centering correction for each $\theta$ bin to put the cross-section at the spectrometer central angle.

- Take the average of the cross-section extracted for each individual $\theta$ bin.

\subsubsection{Acceptance Correction}

The acceptance of the spectrometer represents the probability that a scattering event coming from a point will be detected. This is one of the dominant sources of uncertainties in determining the cross section. For HRS the acceptance is normally defined by various apertures in the magnet system and the beam pipe. The HRS acceptance is a function of electron momentum, the in-plane angle, the out of plane angle and the target y. The in-plane and out of plane angles can be related to the full scattering angle by the following formula:

$$
\theta=\cos ^{-1}\left(\frac{\cos \theta_{0}-y_{t a r}^{\prime} \sin \theta_{0}}{\sqrt{x_{t a r}^{\prime 2}+y_{t a r}^{\prime 2}+1}}\right)
$$

As the inclusive cross section is independent of azimuthal angle, the acceptance is only a function of two variables $\delta$ and $\theta$ i.e. $A(\delta, \theta)$. In order to calculate $A(\delta, \theta)$, MC events were generated uniformly with the range of $\pm 80 \mathrm{mrad}$ in $x_{t a r}^{\prime}, \pm 50 \mathrm{mrad}$ in $y_{\text {tar }}^{\prime}$ and $\pm 6 \%$ in $\delta$ using a model of the spectrometer and take the ratio of the number of events accepted to the number of events generated in the phase space. i.e.

$$
A(\delta, \theta)=\frac{N_{a c c}\left(\delta_{G}, \theta_{G}\right)}{N_{G}\left(\delta_{G}, \theta_{G}\right)},
$$

where $N_{a c c}\left(\delta_{G}, \theta_{G}\right)$ is the number accepted and $N_{G}\left(\delta_{G}, \theta_{G}\right)$ is the number of events generated in each $\left(\delta_{G}, \theta_{G}\right)$ bin. The subscripts gen denote the kinematics as generated. When using the uniform generator, if the resolution is stable across the acceptance, one can use either the generated events that are accepted or the reconstructed events that are accepted. But if the cross section is changing with $\theta$, the net number of events that migrate into a bin 

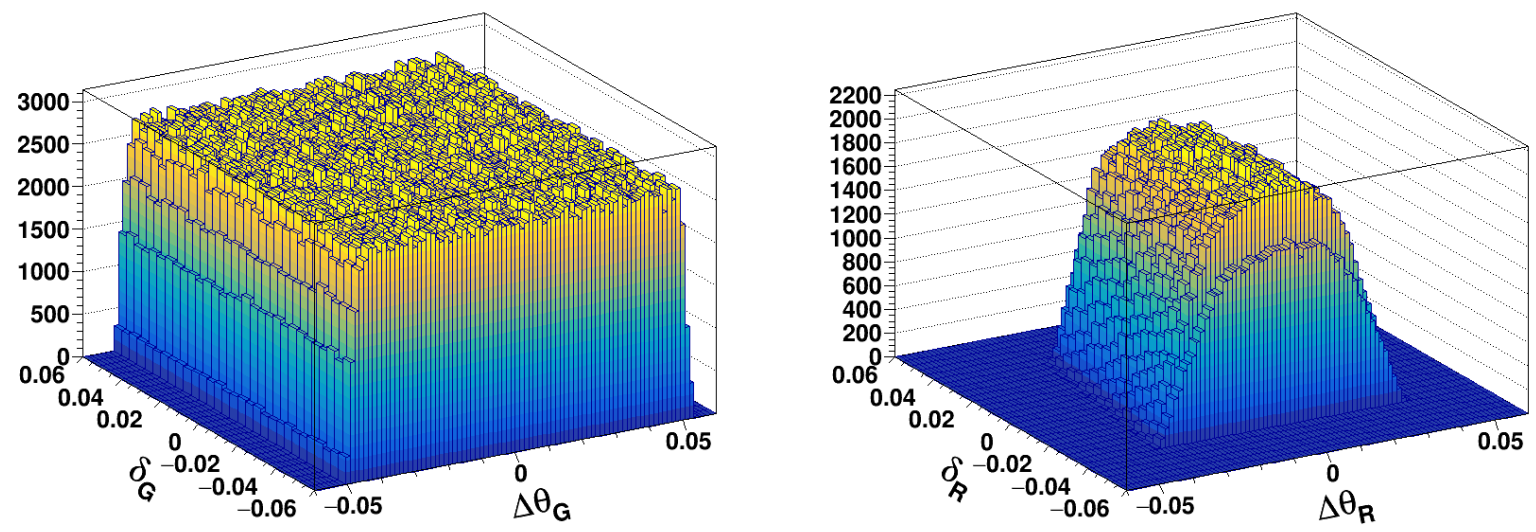

Figure 4.8. Left: Generated $\delta$ vs generated $\Delta \theta$. Right: reconstructed $\delta$ vs reconstructed $\Delta \theta$ in $\delta$ - $\Delta \theta$ space.

depends on the relative cross section of the neighboring bin. So, in this case, reconstructed events that are accepted were used to take into account the bin migration. i.e.
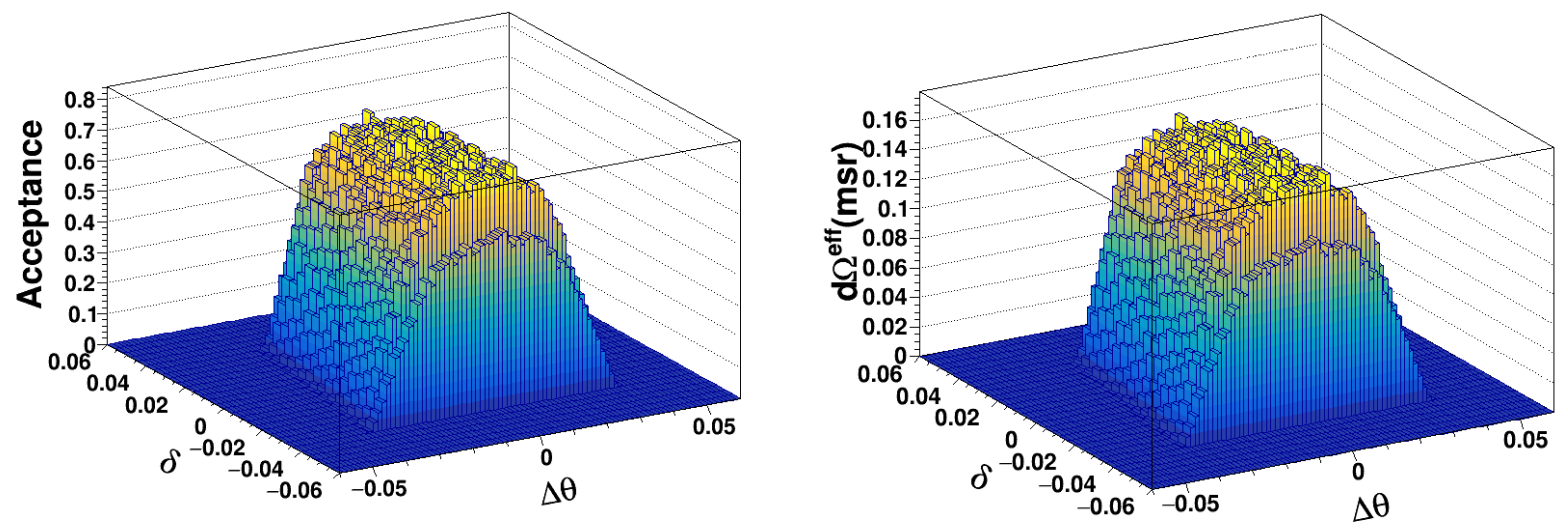

Figure 4.9. Left: HRS acceptance in $\delta-\Delta \theta$ space. Right: HRS effective solid angle plotted in $\delta$ - $\Delta \theta$ space.

$$
A(\delta, \theta)=\frac{N_{R}\left(\delta_{R}, \theta_{R}\right)}{N_{G}\left(\delta_{G}, \theta_{G}\right)},
$$


where the subscripts $R$ denote the kinematics as reconstructed. The acceptance here depends on the solid angle $\Delta \Omega_{\text {gen }}(\theta)$ into which events are generated. Hence, the effective solid angle coverage for each $2-\mathrm{D}$ bin is given by

$$
\begin{aligned}
\Delta \Omega_{e f f}(\delta, \theta) & =A(\delta, \theta) \Delta \Omega_{g e n}(\theta) \\
& =\frac{N_{G}(\theta) \times \Delta x_{t a r}^{\prime} \times \Delta y_{\text {tar }}^{\prime}}{N_{\text {gen }}^{\text {total }}},
\end{aligned}
$$

where $N_{G}(\theta)$ is the number of generated events in each $\theta$ bin and $N_{\text {gen }}^{\text {total }}$ represents the total number of events generated. Here, $\Delta \Omega_{e f f}(\delta, \theta)$ is independent of the size of $\Delta \Omega_{g e n}(\theta)$. The
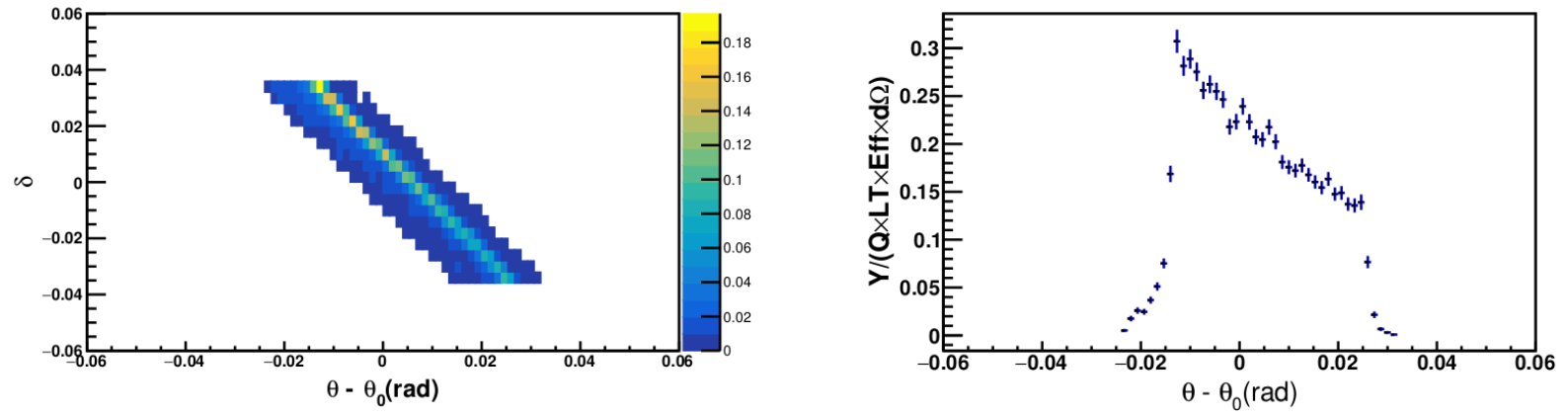

Figure 4.10. Left: Acceptance corrected charge normalized yield $\delta$ - $\Delta \theta$ space. Right: Acceptance corrected charge normalized yield in $\Delta \theta$.

$\Delta \Omega_{\text {eff }}$ distribution determined from HRS model for kinematics K3-7 is shown in Fig 4.9. The solid angle of the HRS is about 6 msr. This is slightly reduced for an extended target. The next step is to divide the efficiency corrected yield defined in the Fig. 4.7 by the effective solid angle. The first 2-D histogram of $\delta$ vs $\theta$ in Fig. 4.10 represents the acceptance corrected charge normalized yield in $\delta-\Delta \theta$ space. Then a summation over $\delta$ bins has been made to get bins just in $\theta$ as shown in the right plots of Fig. 4.10.

\subsubsection{Bin-Centering Correction and Averaging}

If the cross section linearly depends on the scattering angle, the extracted cross section in each bin can simply be averaged. But in this case the cross section varies non-linearly 
across the angular acceptance because of the cross section. So to get an average of the cross sections in each $\theta$, the $\theta$ dependence of the cross section must be corrected. The process of removing of the $\theta$ dependence of cross section by reproducing it at the central angle of the spectrometer is called $\theta$-bin centering. To do this the following correction has been applied:

$$
\left[\frac{d \sigma(\theta)}{d \Omega}\right]_{B C, i}=\frac{d \sigma\left(\theta_{i}\right)}{d \Omega} \frac{\sigma_{m o d}(\theta)}{\sigma_{m o d}\left(\theta_{i}\right)}
$$

where $\theta_{i}$ is the angle for $i^{t h}$ bin and $\sigma_{\text {mod }}$ is the value of a cross section model. This correction

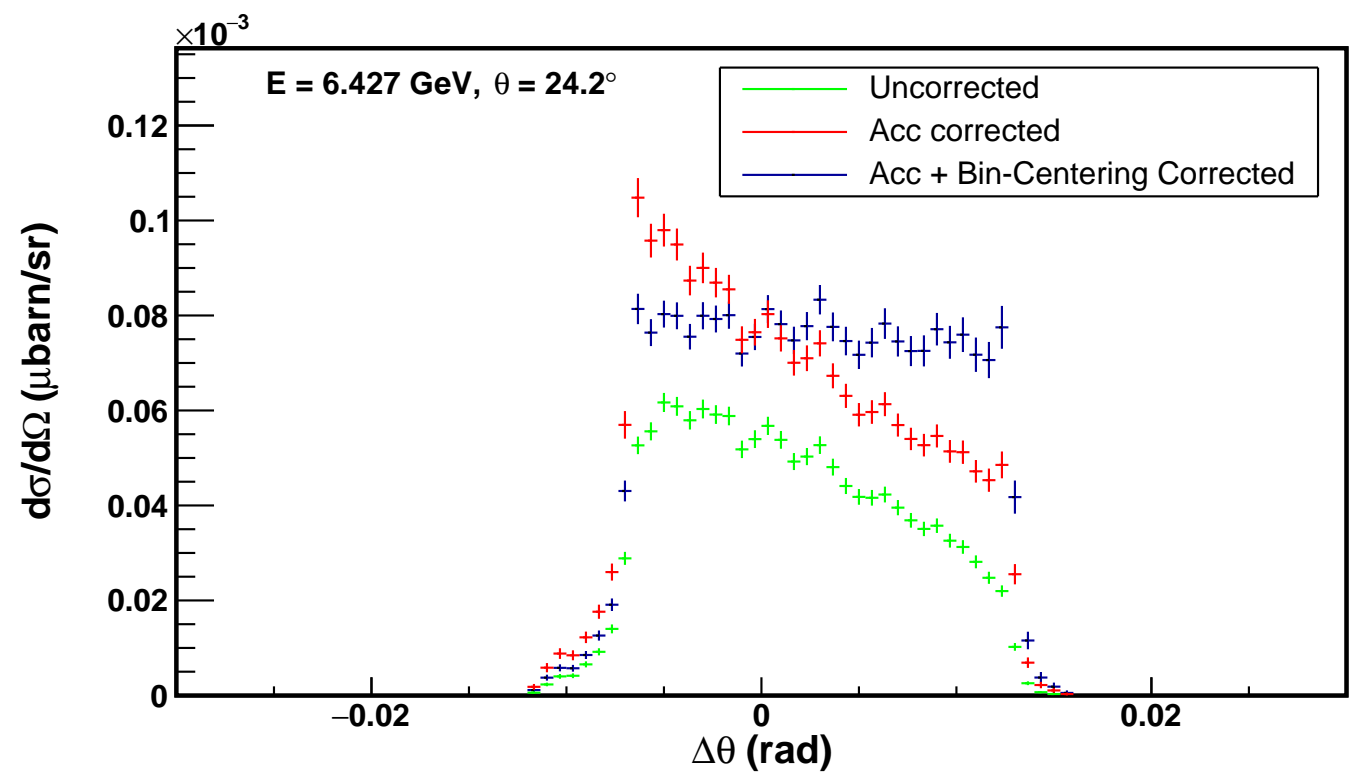

Figure 4.11. Extracted cross section for each $\Delta \theta$.

should apply after subtracting all background and all other corrections having $\theta$ dependence including radiative corrections. Finally, the bin centered cross section can be averaged over the $\theta_{i}$ to give the measured cross section at the spectrometer central angle as shown in Fig. 4.12. In Fig. 4.11 the green points represent the uncorrected cross sections, the red points are the cross sections after applying acceptance correction, and the blue points are the cross sections after applying both acceptance and bin centering cross section. The statistical uncertainties for both LH2 data, background subtracted events, and acceptance correction 


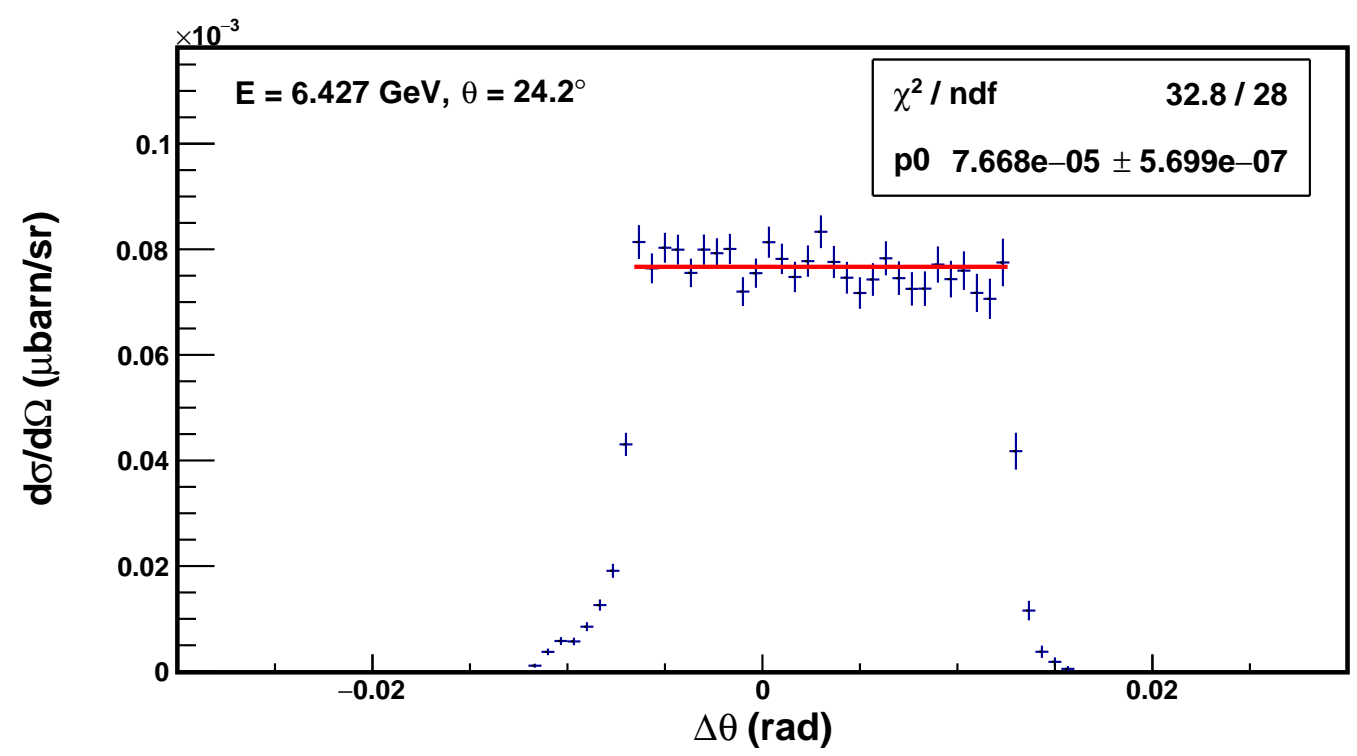

Figure 4.12. Extracted cross section in each $\Delta \theta$.

uncertainties due to statistical errors on the Monte Carlo are included. Table 4.5 shows the comparison of cross section extracted from the ratio and acceptance correction methods. For six out of eight points, the average cross section agree better than $0.5 \%$.

\section{Table 4.5}

Tabular comparison of extracted cross section from MC ratio and acceptance correction methods.

\begin{tabular}{|l|l|l|l|l|l|}
\hline Kinematic & $\begin{array}{l}\theta_{0} \\
(\text { degrees })\end{array}$ & $\begin{array}{l}p_{0} \\
(\mathrm{GeV})\end{array}$ & $\begin{array}{l}(d \sigma / d \Omega)_{\text {Ratio }} \\
(\mu b / s r)\end{array}$ & $\begin{array}{l}(d \sigma / d \Omega)_{\text {Acc.cor. }} \\
(\mu b / s r)\end{array}$ & $\begin{array}{l}\% \text { diff. } \\
(\%)\end{array}$ \\
\hline k1-1 & 42.0 & 1.366 & $1.449 \mathrm{E}-03$ & $1.463 \mathrm{E}-3$ & -0.95 \\
\hline K3-4 & 24.2 & 3.962 & $7.740 \mathrm{E}-05$ & $7.668 \mathrm{E}-05$ & 0.93 \\
\hline K3-6 & 30.9 & 3.224 & $1.108 \mathrm{E}-05$ & $1.093 \mathrm{E}-05$ & 1.30 \\
\hline K3-7 & 37.0 & 2.672 & $2.898 \mathrm{E}-06$ & $2.900 \mathrm{E}-06$ & -0.10 \\
\hline K3-8 & 44.5 & 2.145 & $8.192 \mathrm{E}-05$ & $8.265 \mathrm{E}-05$ & -0.88 \\
\hline K4-9 & 30.9 & 3.685 & $1.285 \mathrm{E}-06$ & $1.282 \mathrm{E}-06$ & 0.23 \\
\hline K4-10 & 34.4 & 3.259 & $5.871 \mathrm{E}-07$ & $5.862 \mathrm{E}-07$ & 0.15 \\
\hline K4-11 & 42.0 & 2.531 & $1.543 \mathrm{E}-07$ & $1.579 \mathrm{E}-07$ & -2.4 \\
\hline
\end{tabular}




\section{CHAPTER 5 \\ RESULTS}

The elastic cross sections measured by the GMp experiment are shown in Fig. 5.1. This shows the measured cross section divided by the cross section calculated assuming dipole form factors for both $G_{E}$ and $G_{M}$ i.e.

$$
\left(\frac{d \sigma}{d \Omega}\right)_{\text {dipole }, 1 \gamma}=\left(\frac{d \sigma}{d \Omega}\right)_{m o t t} \frac{G_{D}^{2}\left(Q^{2}\right)\left(\varepsilon+\tau \mu_{p}^{2}\right)}{\varepsilon(1+\tau)}
$$

These data were taken at smaller $\varepsilon$ than the SLAC data of Sill. In this dissertation the final results for five of the kinematic points from the Fall 2016 run of GMp are presented. As well as, the preliminary results for two data points taken with the RHRS. Final analysis of the RHRS data will be forthcoming once the optics optimization has been completed. Additionally, the data points at $Q^{2}=1.8,9.0,12.5,15.7,5.9,6.9$ and $9.0 \mathrm{GeV}^{2}$ from Fall 2016 originally analyzed by L. Ou [58], have been included, but have been updated to include the following corrections:

1. increased Q2 quad in the simulation field based upon the study of Ref. [60], which has a small impact on the acceptance on the order of few tenths of a percent,

2. final determination of the target $\mathrm{z}$ position offset from hall center was studied in Section 4.0.1.3 and implemented in MC which has a small effect on the acceptance,

3. the absolute current offset of $0.13 \mu \mathrm{A}$ found in Section 3.4.

The data points at $Q^{2}=1.66,1.51,1.10$ and $0.66 \mathrm{GeV}^{2}$ are from the Spring 2015 run period and were analyzed by Y. Wang [61]. The low $Q^{2}$ data collected provide a benchmark for the measurements, as the cross section is known well at low $Q^{2}$.

It is clear from Fig. 5.1 that the GMp data at $Q^{2}>6 \mathrm{GeV}^{2}$ provide a significant improvement in precision. For instance, at $Q^{2}$ of $9.8 \mathrm{GeV}^{2}$ the elastic cross section measured by this 


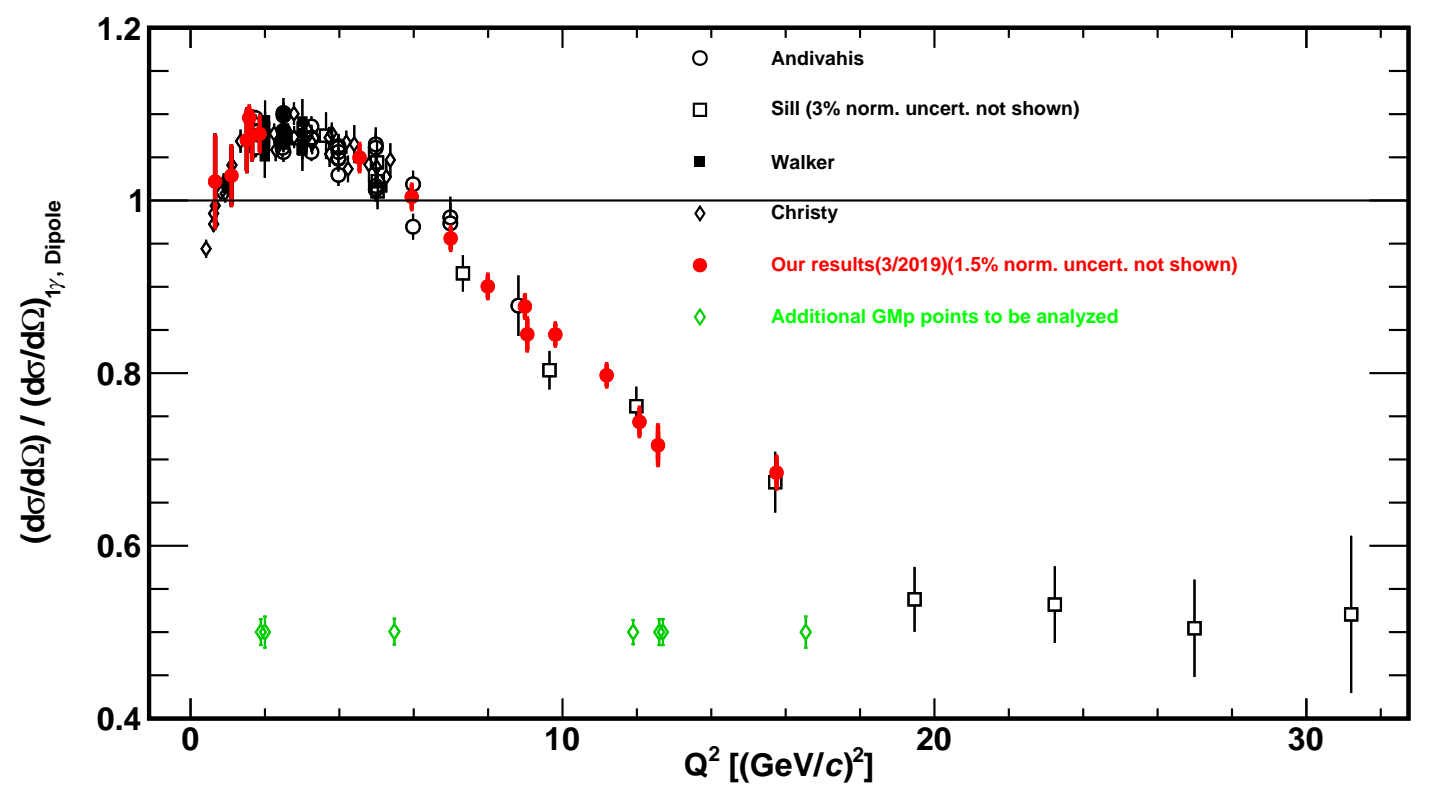

Figure 5.1. The results of extracted $e-p$ cross sections divided by the cross section calculated in the OPEA assuming a dipole form for both $G_{E}$ and $G_{M}$ as a function of $Q^{2}$. The inner error bar in GMp data are statistical and outer error bars are combined point-to-point systematic and statistical uncertainties.

experiment has a statistical uncertainty of $0.7 \%$ and a point-to-point systematic uncertainty of $1.3 \%$, whereas the measurement of Sill at $Q^{2}=9.6 \mathrm{GeV}^{2}$ has a statistical uncertainty of $2.4 \%$ and a point-to-point uncertainty of 3.6\%, with GMp experiment reducing the total uncertainty at this $Q^{2}$ almost a factor of 3 . In addition, the normalization uncertainty on the GMp data has been estimated to be $1.8 \%$, while that on the Sill data was estimated to be $3 \%$.

\subsubsection{Model Dependent Extraction of $G_{M}$}

Once the elastic cross section has been determined the magnetic form factor can be extracted. $G_{M}$ versus $Q^{2}$ from a model dependent extraction is shown in Fig. 5.2. This method utilized a parametrization of the $G_{E}$ to $G_{M}$ ratio determined from a fit in the OPEA to the existing cross section data [2] and determined $G_{M}$ from the cross section measured at 
each kinematic from

$$
G_{M}^{2}=\frac{\sigma_{R}}{1+\frac{\varepsilon}{\tau} \frac{G_{E}^{2}}{G_{M}^{2}}}
$$

The results of the form factor measurement for Fall 2016 kinematics are given in Table 5.1. The uncorrelated uncertainties reflect the point-to-point systematic and the statistical uncertainties added in quadrature, while the correlated uncertainty shown as the green histogram at the bottom are calculated assuming a $20 \%$ uncertainty on the $G_{E} / G_{M}$ value used to extract GMp from the cross section data. As given in Section 1.5, in the TPE hypothesis the reduced cross section can be written as

$$
\tilde{\sigma}_{r}=G_{M}^{2}+2 G_{M} \operatorname{Re}\left(\tilde{G_{M}}\right)+\frac{\epsilon}{\tau}\left[G_{E}^{2}+\frac{4 \tau^{2}}{M^{2}} \operatorname{Re}\left(\tilde{F}_{3}\right)\left(G_{M}+\frac{1}{\tau} G_{E}\right)+2 G_{E} \operatorname{Re}\left(\tilde{G_{M}}\right)\right]
$$

If the TPE hypothesis is correct, then the intercept extracted from the Rosenbluth separations is actually $\left(G_{M}\right)^{2}+2 \times G_{M} \times \operatorname{Re}\left(\delta G_{M}\right)$. Additionally, the GMp data set provides significant additional constraints to extract the $F_{3}$ form factor and the complex contributions to $G_{E}\left(\delta \mathrm{G}_{E}\right)$ and $G_{M}\left(\delta \mathrm{G}_{M}\right)$.

\subsubsection{Rosenbluth Separation}

The model independent $G_{M}$ can be extracted by using a Rosenbluth separation. This method has following advantages:

1. it provides a check of the consistency of the $\varepsilon$ dependence of data sets,

2. it provides a model independent extraction of the form factor within the OPEA,

3. it also illustrates the impact of the GMp data on our knowledge of form factor at large $Q^{2}$. 
Table 5.1

Summary of magnetic from factors relative to the dipole form factor extracted utilizing the $G_{E}$ to $G_{M}$ ratio using a parametrization from the fits to the existing data.

\begin{tabular}{|c|c|c|}
\hline$Q^{2}\left(\mathrm{GeV}^{2}\right)$ & $G_{M}^{p} /\left(\mu G_{D}\right)$ & Correlated un. \\
\hline 1.57 & $1.0631 \pm 6.378 \mathrm{e}-03$ & 0.0146 \\
\hline 4.54 & $1.0225 \pm 7.668 \mathrm{e}-03$ & 0.0080 \\
\hline 5.94 & $1.002 \pm 7.013 \mathrm{e}-03$ & 0.0051 \\
\hline 6.99 & $9.805 \mathrm{e}-01 \pm 6.864 \mathrm{e}-03$ & 0.0031 \\
\hline 7.99 & $9.528 \mathrm{e}-01 \pm 7.146 \mathrm{e}-03$ & 0.0017 \\
\hline 9.00 & $9.432 \mathrm{e}-01 \pm 7.074 \mathrm{e}-03$ & 0.0016 \\
\hline 9.81 & $9.259 \mathrm{e}-01 \pm 6.944 \mathrm{e}-03$ & 0.0016 \\
\hline 11.3 & $8.978 \mathrm{e}-01 \pm 7.190 \mathrm{e}-03$ & 0.0005 \\
\hline 1.86 & $1.051 \mathrm{e}+00 \pm 9.992 \mathrm{e}-03$ & 0.0110 \\
\hline 9.05 & $9.228 \mathrm{e}-01 \pm 1.015 \mathrm{e}-02$ & 0.0008 \\
\hline 12.5 & $8.502 \mathrm{e}-01 \pm 1.360 \mathrm{e}-02$ & 0.0002 \\
\hline 15.7 & $8.309 \mathrm{e}-01 \pm 1.121 \mathrm{e}-02$ & $3.02 \mathrm{e}-05$ \\
\hline 12.1 & $8.667 \mathrm{e}-01 \pm 9.534 \mathrm{e}-03$ & 0.0002 \\
\hline
\end{tabular}

The results from this analysis will be compared with the results obtained from the model dependent analysis. Examining Fig 1.10 it can be seen that there are several previous measurements at $Q^{2}$ close to $1.5,5.0,5.8,7.0$ and $9.1 \mathrm{GeV}^{2}$. As few of those were taken at exactly the same $Q^{2}$, a correction factor was applied to move all data to a common $Q^{2}$, utilizing the model as

$$
\sigma_{R}\left(Q_{c}^{2}, \varepsilon\right)=\sigma_{R}\left(Q^{2}\right) \times \frac{\sigma_{R}^{M o d}\left(Q_{c}^{2}, \varepsilon\right)}{\sigma_{R}^{M o d}\left(Q^{2}, \varepsilon\right)}
$$

Here, $\sigma^{M o d}$ is the value obtained from the fit to previous cross section data as discussed in Section 1.6 and $Q^{2}\left(Q_{c}^{2}\right)$ represent the measured (central) $Q^{2}$.

In order to perform Rosenbluth separations at a particular $Q^{2}$, each separation must contain three different $\varepsilon$ points. The plots of reduced cross section normalized by $G_{D}^{2}$ versus $\varepsilon$ at the five different $Q^{2}$ mentioned above along with the best fit straight line are shown in Figures 5.3 through 5.7. The uncertainty on each point represents the statistical and point- 


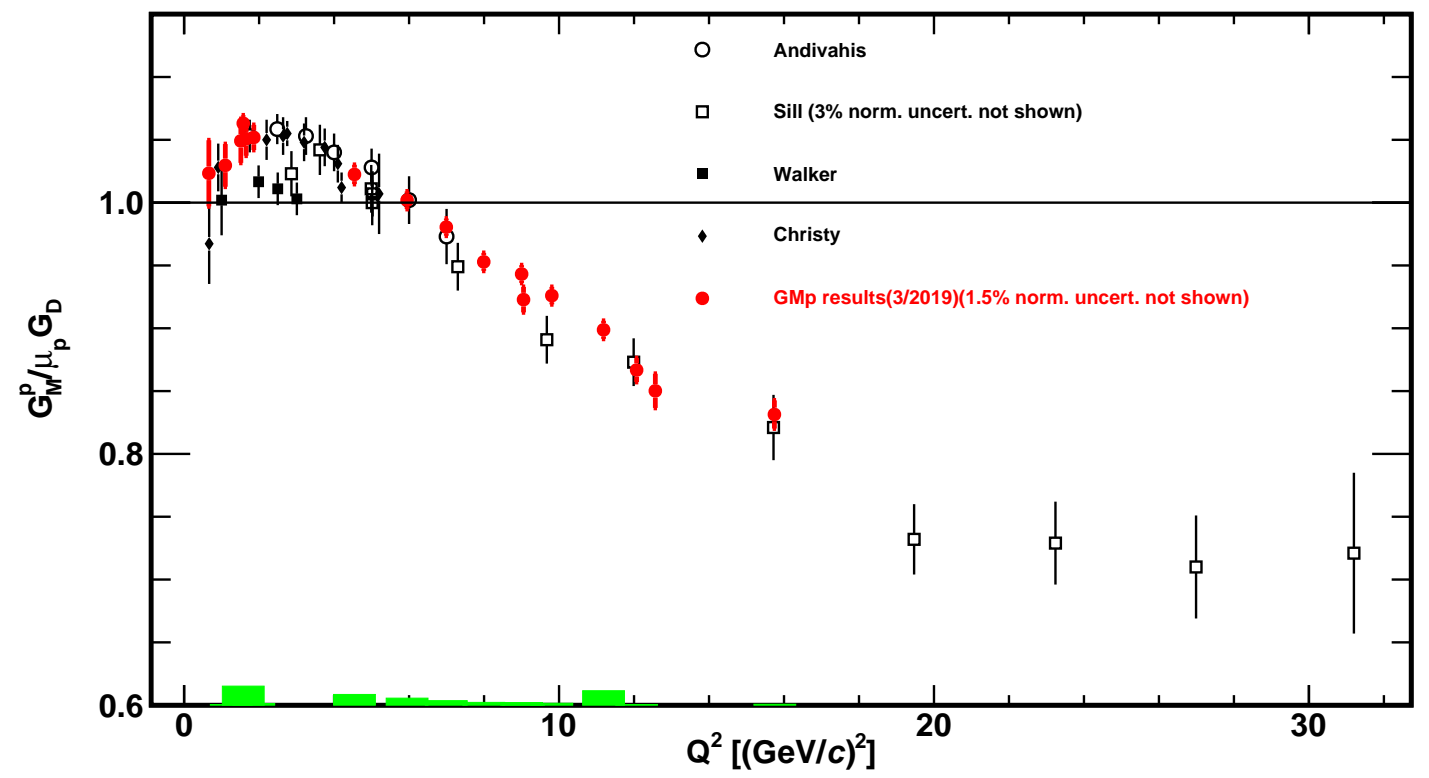

Figure 5.2. The results of model dependent extraction of GMp as a function of $Q^{2}$. The uncorrelated uncertainties reflect the point-to-point systematic and the statistical uncertainties added in quadrature, while the correlated uncertainty shown as the green histogram at the bottom are calculated assuming a $20 \%$ uncertainty on the $G_{E} / G_{M}$ value used to extract GMp from the cross section data.

to-point systematic uncertainties added in quadrature. Currently no relative normalizations between the data sets have been applied. Table 5.2 lists the electric and magnetic form factors relative to the dipole form factor along with their uncertainties extracted from the Rosenbluth separation.

Table 5.2

Summary of the Rosenbluth method form factors relative to the dipole form factor.

\begin{tabular}{|l|l|l|l|}
\hline $\begin{array}{l}Q^{2} \\
\left(\mathrm{GeV}^{2}\right)\end{array}$ & $G_{M}^{p} /\left(\mu_{p} G_{D}\right)$ & $G_{E}^{p} /\left(G_{D}\right)$ & $\mu_{p} G_{E}^{p} / G_{M}^{p}$ \\
\hline 1.702 & $1.049 \pm 0.007$ & $0.983 \pm 0.045$ & $0.936 \pm 0.038$ \\
\hline 5.001 & $1.017 \pm 0.008$ & $0.946 \pm 0.135$ & $0.932 \pm 0.081$ \\
\hline 5.831 & $0.994 \pm 0.010$ & $1.053 \pm 0.187$ & $1.059 \pm 0.123$ \\
\hline 7.073 & $0.966 \pm 0.014$ & $1.120 \pm 0.294$ & $1.159 \pm 0.207$ \\
\hline 9.128 & $0.914 \pm 0.019$ & $1.301 \pm 0.453$ & $1.424 \pm 0.389$ \\
\hline
\end{tabular}




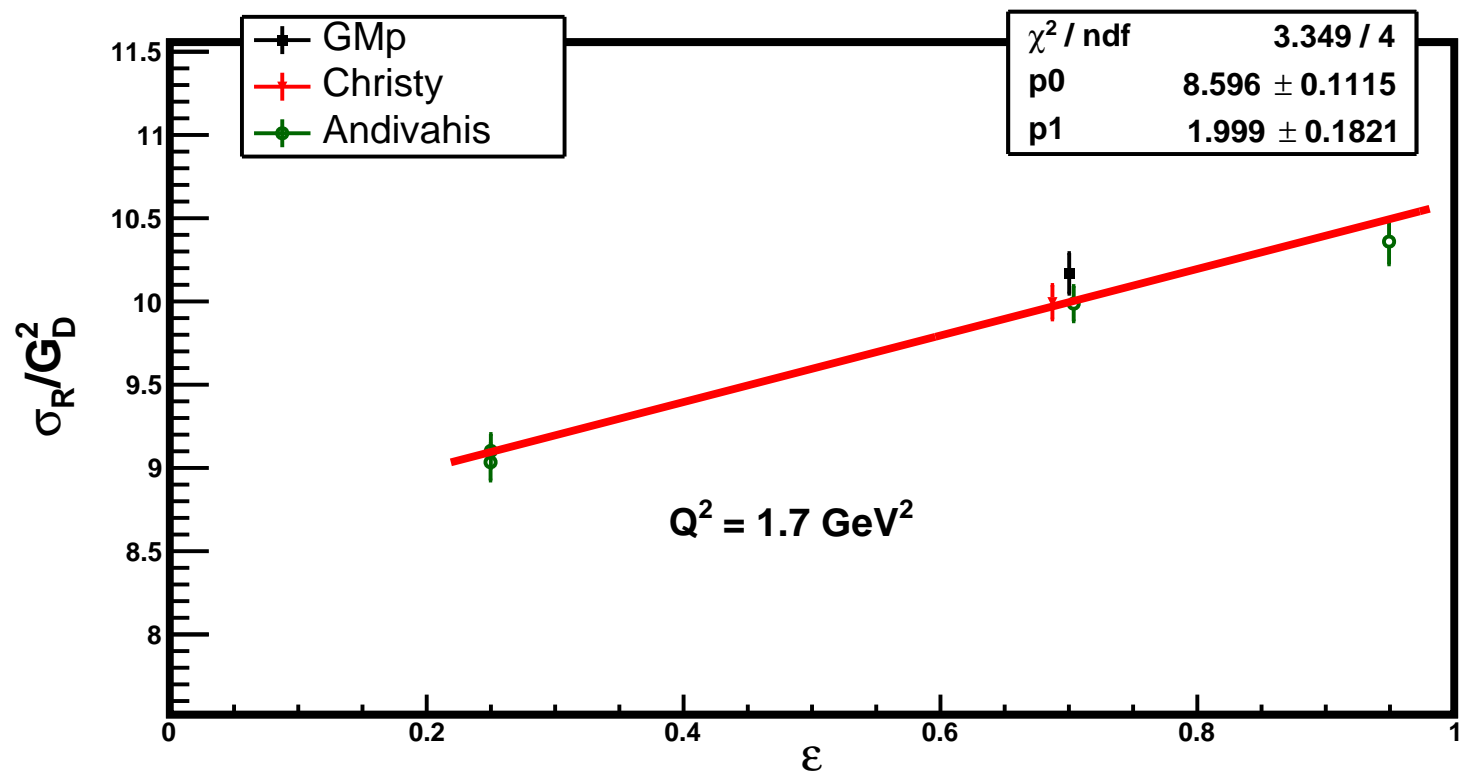

Figure 5.3. Reduced cross section normalized by $G_{D}^{2}$ versus $\varepsilon$ at $Q^{2}=1.7 \mathrm{GeV}^{2}$.

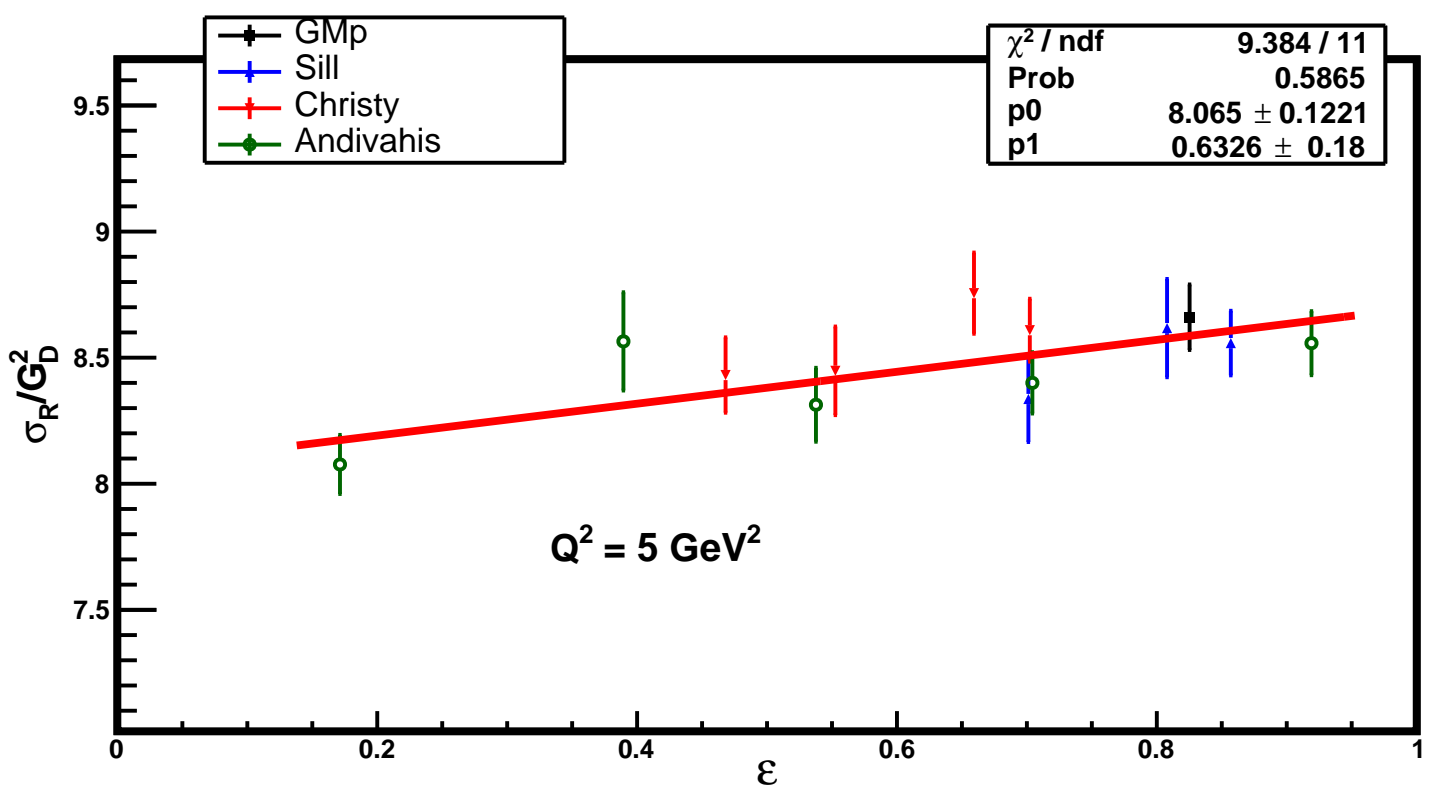

Figure 5.4. Reduced cross section normalized by $G_{D}^{2}$ versus $\varepsilon$ at $Q^{2}=5.0 \mathrm{GeV}^{2}$. 


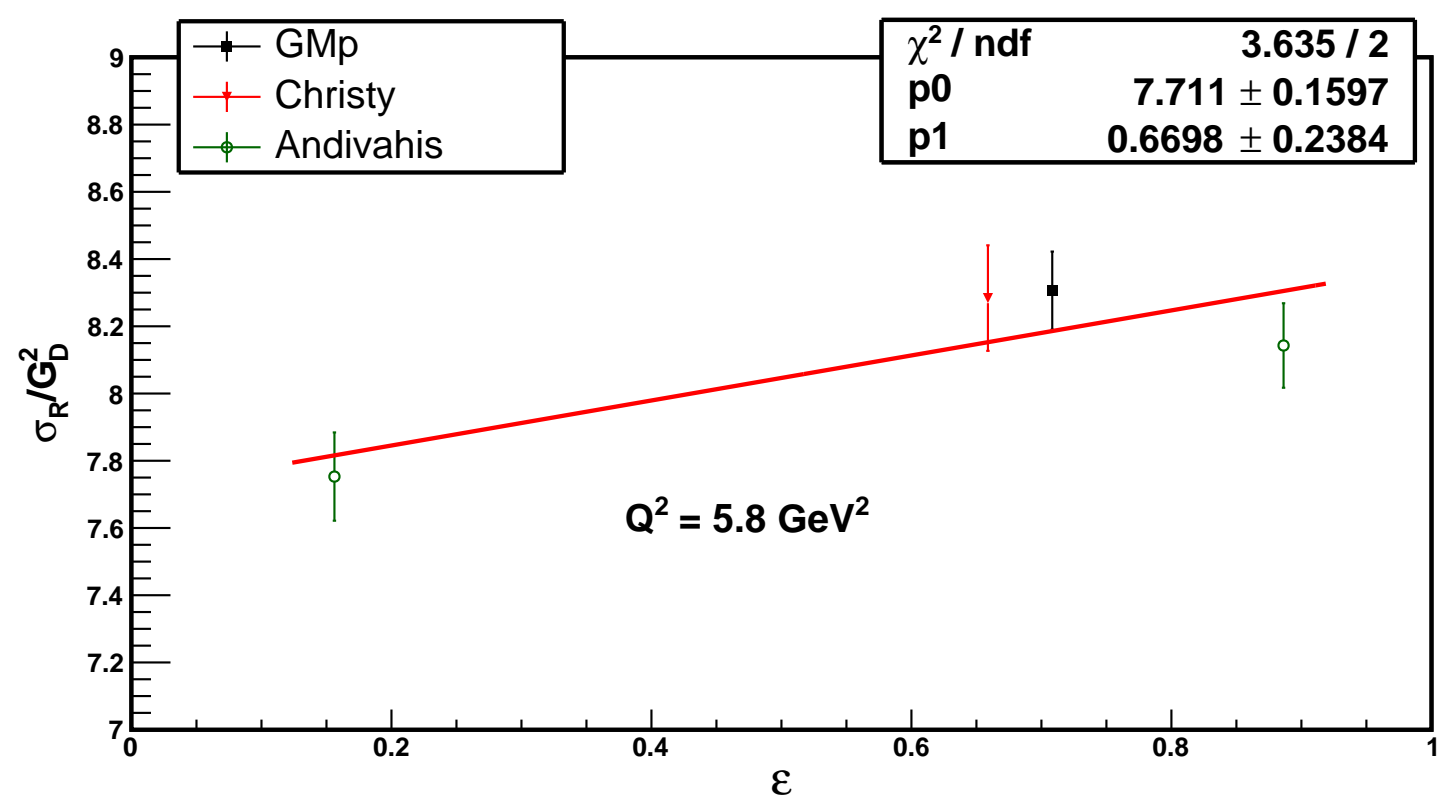

Figure 5.5. Reduced cross section normalized by $G_{D}^{2}$ versus $\varepsilon$ at $Q^{2}=5.8 \mathrm{GeV}^{2}$.

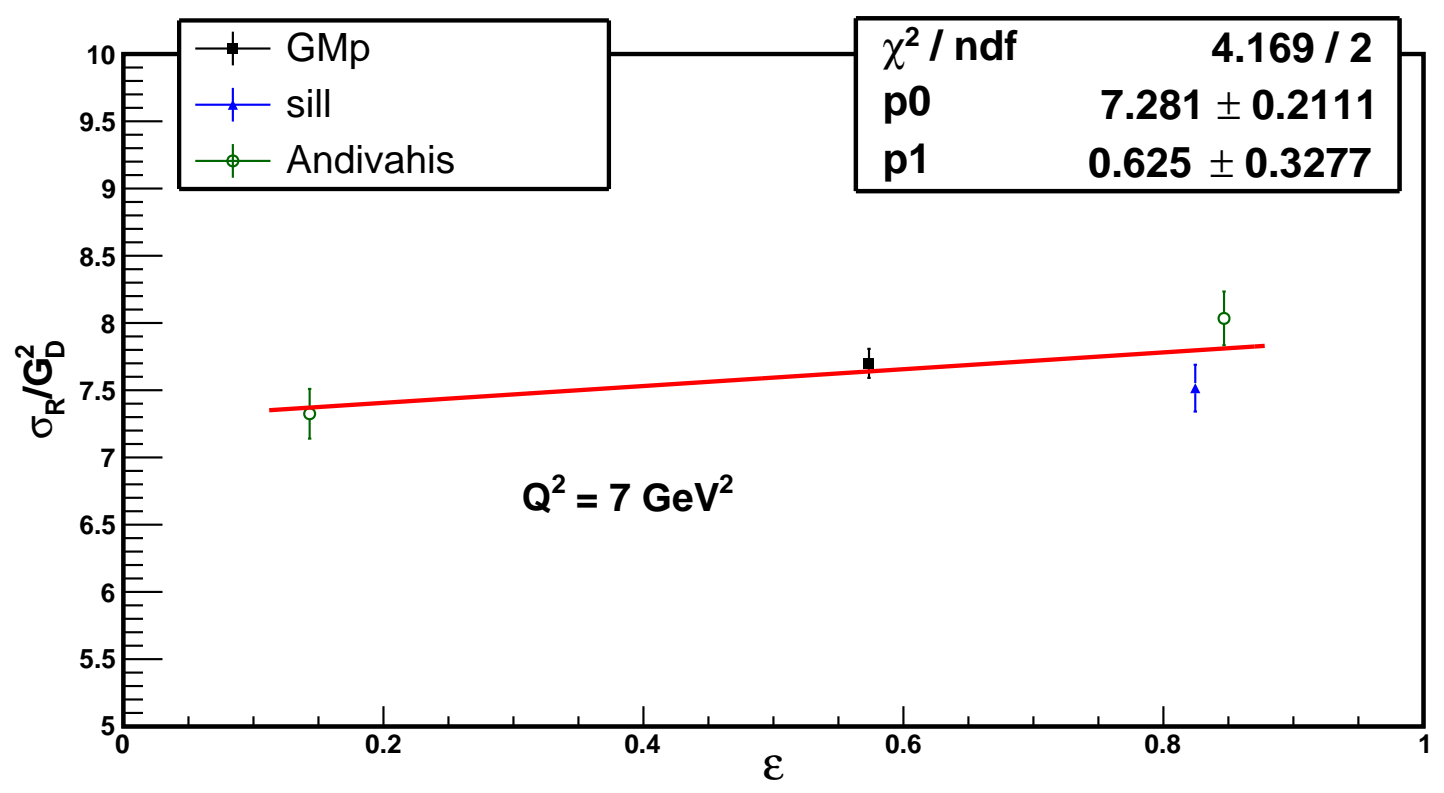

Figure 5.6. Reduced cross section normalized by $G_{D}^{2}$ versus $\varepsilon$ at $Q^{2}=7.0 \mathrm{GeV}^{2}$. 


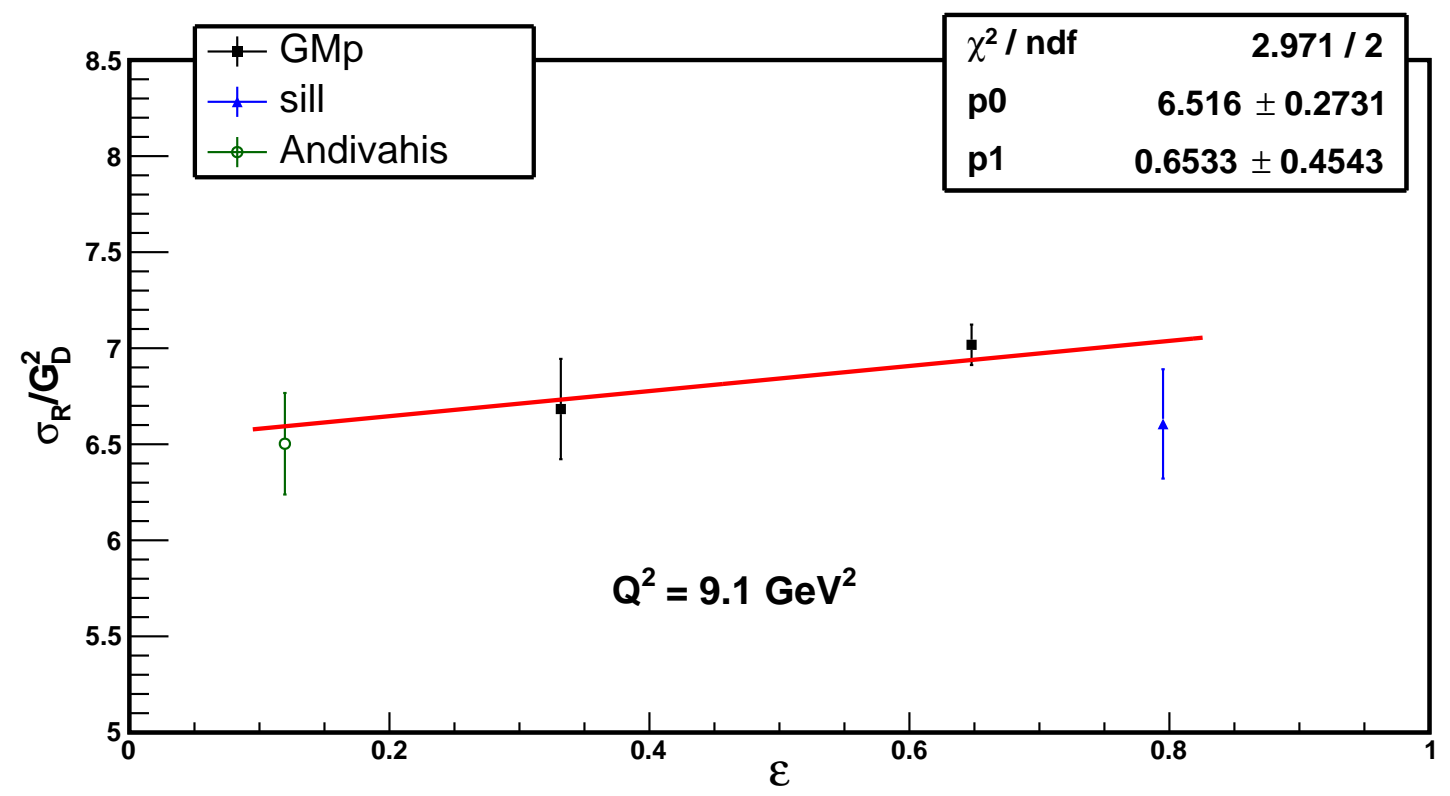

Figure 5.7. Reduced cross section normalized by $G_{D}^{2}$ versus $\varepsilon$ at $Q^{2}=9.1 \mathrm{GeV}^{2}$.

At $Q^{2}$ of $7 \mathrm{GeV}^{2}$ (Fig. 5.6) GMp contributes one out of four points with a factor of two smaller uncertainty than the other data, significantly reducing the uncertainty. At $Q^{2}$ of 9.1 $\mathrm{GeV}^{2}$, there are two GMp points out of four of which one of the data points has an uncertainty four times smaller than the existing data sets. The difference in uncertainties between the two GMp points at this $Q^{2}$ is because the $\varepsilon=0.332$ point was taken with the RHRS and is awaiting final optimization of the spectrometer optics, which will further reduce the uncertainties on the extracted form factors. Inclusion of the GMp data along with that of Sill and Andibhais results in a larger $G_{E} / G_{M}$ ratio with significantly reduced uncertainty, further increasing the tension with the polarization transfer results.

The results for the ratio $G_{E}^{p} / G_{M}^{p}$ from the Rosenbluth separations including the GMp data along with those from previous experiments are shown in Fig. 5.8, where it can be seen that this experiment improved the precision by the factor of two or more further highlighting the discrepancy between Rosenbluth separations and the polarization transfer results. For 


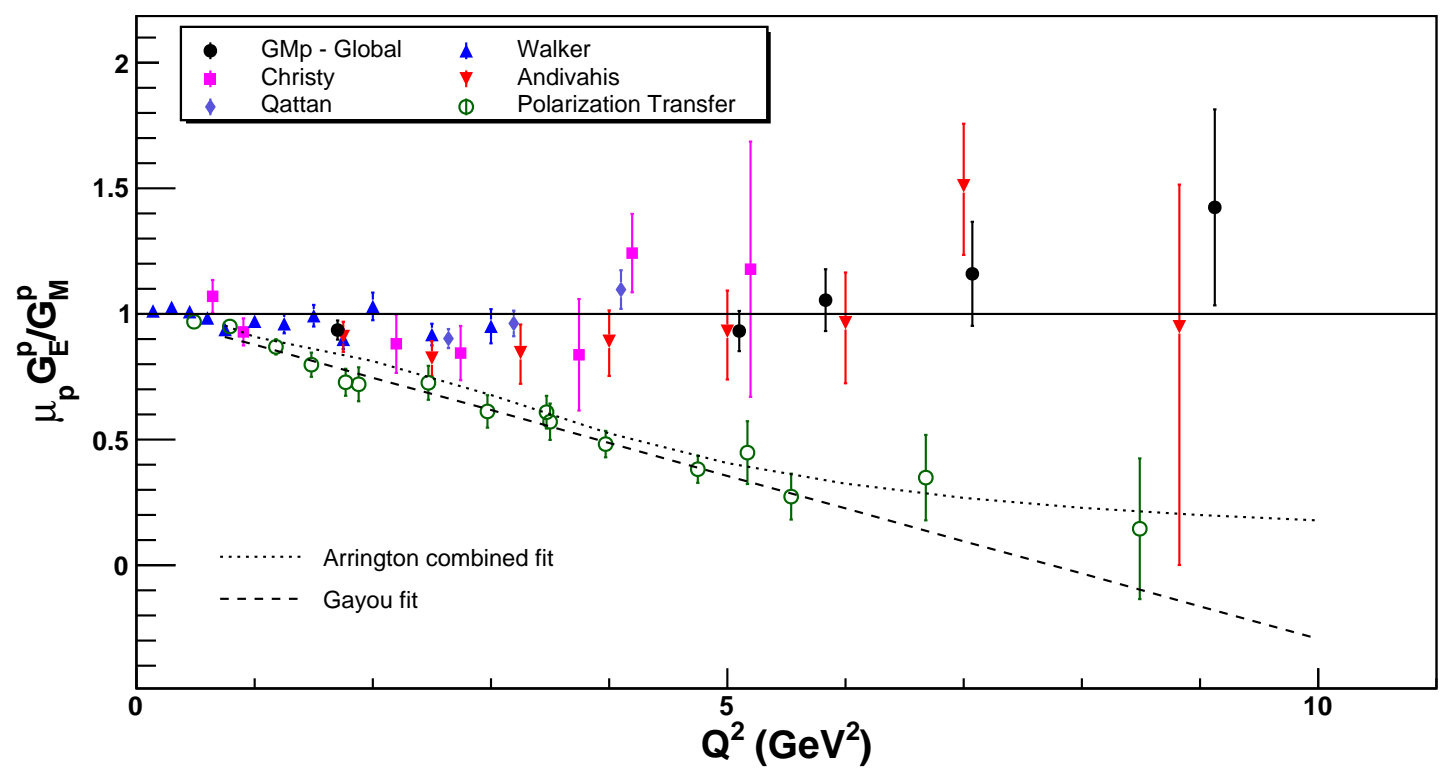

Figure 5.8. Extracted $\mu G_{E}^{p} / G_{M}^{p}$ values from the Rosenbluth separation method (solid symbols) and from the JLab measurements of recoil polarization (open symbols) plotted versus $Q^{2}$ including the Super-Rosenbluth data [16]. The polarization transfer data includes GEp (1) $[17,18], \operatorname{GEp}(2)[19,20]$ and GEp (3) [21] data. The GMp-Global at $Q^{2}$ of $5 \mathrm{GeV}^{2}$ has been offset by $0.1 \mathrm{GeV}^{2}$ for clarity. The fits in the figure are from Gayou [22] and Arrington [23].

instance at $Q^{2}$ of $9.1 \mathrm{GeV}^{2}$ the GMp-Global uncertainty is about 0.388 whereas the Andivahis data at $Q^{2}$ of $8.8 \mathrm{GeV}^{2}$ has uncertainty 0.757 . The reduction in uncertainty at $9.1 \mathrm{GeV}^{2}$ is mostly coming from GMp data, whereas at other $Q^{2}$ the reduction is due to the combination of uncertainties from other data. Note that at $9 \mathrm{GeV}^{2}$, the error bar went significantly down as GMp added two points with significantly better uncertainty overall than existing data. Going from a two point separation to a four point separation with reduced uncertainty on the new data significantly reduces the uncertainty on the form factors at that $Q^{2}$.

Fig. 5.9 shows the comparison of GMp extracted by using the model dependent method and GMp-Global data extracted by using the Rosenbluth separation method. This plot shows that the GMp extracted data using both methods are in good agreement. 


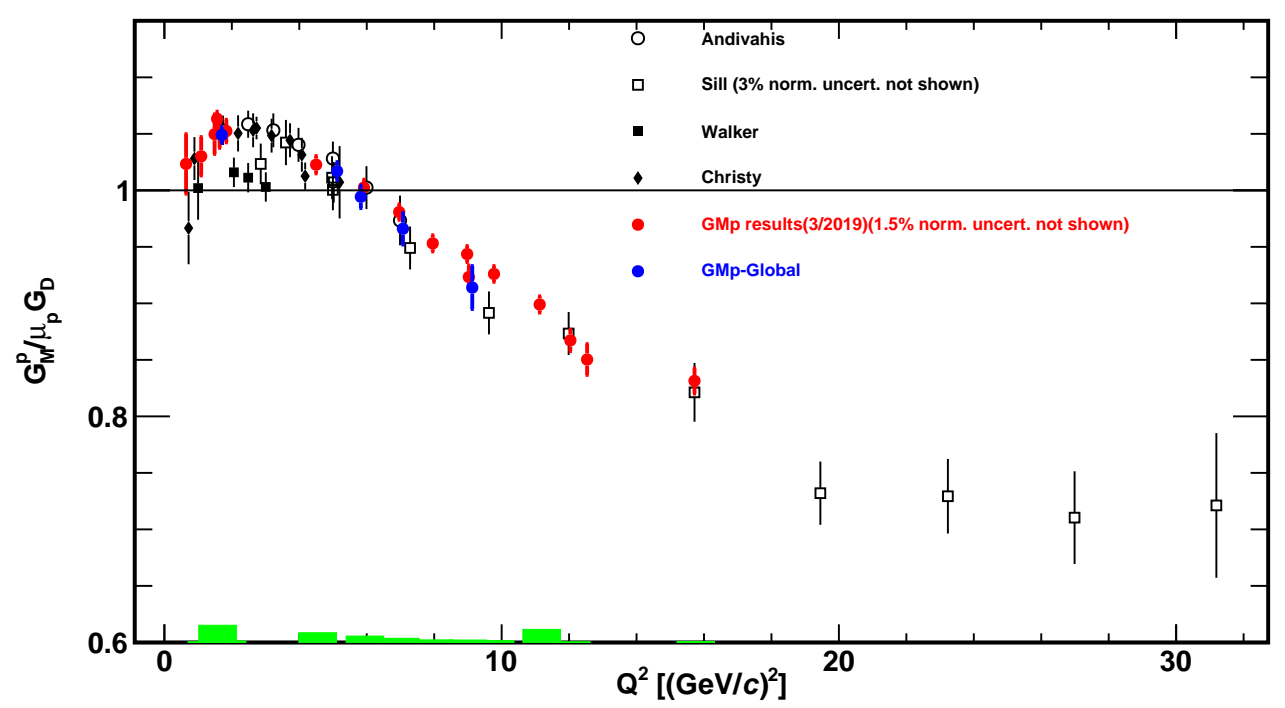

Figure 5.9. Experimental $G_{M}^{p} / \mu_{p} G_{D}$ values versus $Q^{2}$. The red solid circles are the values extracted from the model dependent parametrization of the existing data (see Section 1.6) in single photon approximation, whereas the blue points were extracted from Rosenbluth separation also in single photon approximation.

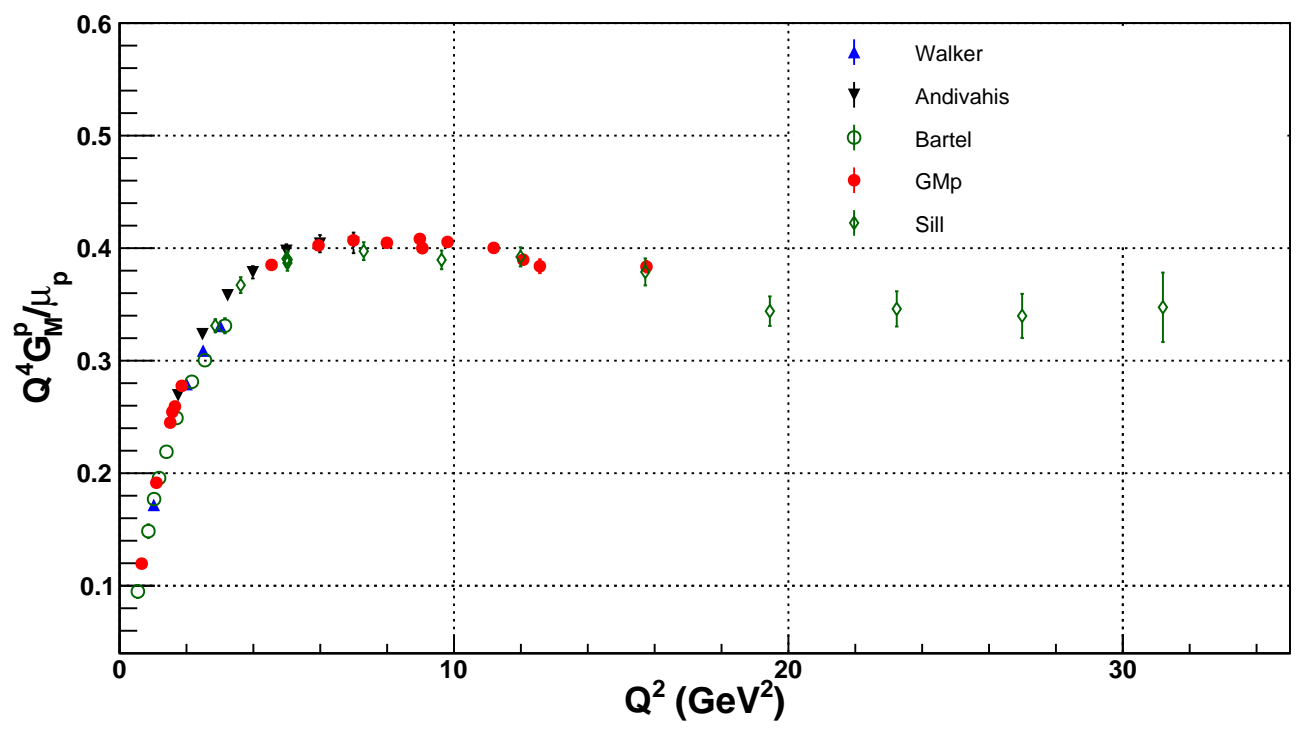

Figure 5.10. $Q^{4} G_{M}^{p} / \mu_{p}$ versus $Q^{2}$ showing asymptotic behavior of the proton magnetic form factor. The red points are the GMp experiment data extracted from the model dependent parametrization of the existing data whereas other data are the existing data. 
The Fig. 5.10 shows $Q^{4} G_{M}^{p} / \mu_{p}$ as a function of $Q^{2}$. The plot shows the proton magnetic form factor approximately satisfies the pQCD scaling prediction up to the highest $Q^{2}$ of the GMp data, but have slow variations at high $Q^{2}$. 


\section{CHAPTER 6 CONCLUSION}

The GMp experiment was one of the first two experiments to run in Jefferson Lab experimental Hall A after the $12 \mathrm{GeV}$ energy upgrade. This experiment performed a high precision measurement of the proton $e p$ elastic scattering cross section to determine the magnetic form factor over a wide range of $Q^{2}$ from below $1 \mathrm{GeV}^{2}$ to $16.5 \mathrm{GeV}^{2}$. This dissertation utilized all GMp kinematics from Fall 2016 and presented the results for the form factors extracted from two methods. In the Rosenbluth separation method, the extracted cross sections obtained from this experiment and previous measurements were combined to extract the both magnetic form factor $\left(G_{M}\right)$, and electric form factor $\left(G_{E}\right)$ at different $Q^{2}$ up to $Q^{2}=9 \mathrm{GeV}^{2}$. The second, model dependent method utilized the ratio $\mu G_{E} / G_{M}$ from a fit to the existing world data to extract $G_{M}$ from each cross section measurements with both $G_{M}$ results are in good agreement and with significantly reduced uncertainties to existing data. The results from the Spring 2016 kinematics are still being finalized.

The measurement of nucleon form factors has provided significant insight into the substructure of the nucleon. The existing form factor data at low $Q^{2}$ cannot be treated by pQCD. On the other hand in the high $Q^{2}$ regime pQCD is believed to give the correct asymptotic $Q^{2}$ dependence. For the moderate range of $Q^{2}$ of $1-20 \mathrm{GeV}^{2}$, neither pQCD nor a meson description are able to fully describe the data. This range of $Q^{2}$ is very important as the virtual photon becomes more sensitive to the internal quark structure of the nucleon. Also, the electric and magnetic form factors exhibit scaling when extracted using the Rosenbluth method. This sort of behavior was not observed in the polarization transfer technique, which is believed to be an indication that two-photon exchange plays a vital role in the Rosenbluth results. The results of this analysis showed that there is still an inconsistency between Rosenbluth and polarization transfer measurement at $Q^{2}$ around $9 \mathrm{GeV}^{2}$. This data 
can provide additional constraints on contributions from two-photon exchange. This will be the focus of future studies including a global fit with the new precision GMp data. 


\section{REFERENCES}

[1] B. Schmookler, B. Aljawrneh, and T. Gautam. Efficiency Studies for the GMp Experiment, E12-07-108 Technical Note (2018).

[2] J. Arrington, W. Melnitchouk, and J. A. Tjon. Phys. Rev. C 76, 035205 (2007).

[3] W. Bartel, F. W. Busser, and W. R. Dix et al. Nucl. Phys. B58, 429 (1973).

[4] C. Berger, G. Knop V. Burkert, B. Langenbeck, and K. Rith. Phys. Lett. B 35, 87 (1971).

[5] L. Andivahis et al. Phys. Rev. D50, 5491 (1994).

[6] P. N. Kirk and M. Breidenbach et al. Phys. Rev. D 8, 63 (1973).

[7] A. F. Sill, R. G. Arnold, and P. E. Bosted et al. Phys. Rev. D 48, 29 (1993).

[8] M. E. Christy et al. Phys. Rev. C 70, 015206 (2004).

[9] Hall A target configuration. https://hallaweb.jlab.org/12GeV/experiment/E12-07108/Documents/Target/Fall2016/HallATargetConfiguration10-11-16.pdf.

[10] J. Alcorn et al. Nucl. Instrum. Meth. A522, 294 (2004).

[11] P. Monaghan. Study of the $12 \mathrm{C}(\mathrm{e}, \mathrm{e}$ 'p) Reaction in a Correlations Dominant Regime with $\mathrm{Q}^{2}=2.0(\mathrm{GeV} / \mathrm{c})^{2}$ and $\mathrm{X}_{B}>1$, Ph.D. Thesis, Massachusetts Institute of Technology (2008).

[12] Y. Zhang. Measurement of the target single-spin asymmetry in quasi-elastic region from the reaction $3 \mathrm{He}\left(\mathrm{e}, \mathrm{e}^{\prime}\right), \mathrm{Ph}$. D. Thesis, Rutgers, The State University of New Jersey (2013).

[13] B. Schmookler. Target boiling Study. 
[14] Sieve pictures. https://hallaweb.jlab.org/12GeV/experiment/E12-07108/Publications/Technical/Technical_Drawings/sieve_pictures/LHRS_Fall14.jpg.

[15] C. Perdrisat, V. Punjabi, and M. Vanderhaeghen. Prog. Part. Nucl. Phys. 59, 694 (2007), ISSN 0146-6410.

[16] I. A. Qattan et al. Phys. Rev. Lett. 94142301 (2005).

[17] M. K. Jones and K. A. Aniol annd F. T. Baker et al. (The Jefferson Lab Hall A Collaboration). Phys. Rev. Lett. 84, 1398 (2000).

[18] V. Punjabi et. al. Phys. Rev. C 71,(2005) 055202.

[19] O. Gayou et al. hys. Rev. Lett. 88, 092301 (2002) .

[20] A.J.R Puckett et al. Phys. Rev. 85, 045203 (2012).

[21] A.J.R Puckett et al. Phys. Rev. Lett. 104, 242301 (2010).

[22] O. Gayou et al. Phys. Rev. C 64, 038202 (2001).

[23] J. Arrington. Phys. Rev. C 69, 022201(R) (2004).

[24] R. Hofstadter. Rev. Mod. Phys. 28, 214 (1956).

[25] R. G. Sachs. Phys. Rev. 126, 2256 (1962).

[26] H. Frauenfelder and E.M. Henley. Subatomic Physics, (Prentice-Hall Inc., New Jersey, 1974).

[27] P.G. Blunden, W. Melnitchouk, and J.A. Tjon. Phys. Rev. Phys. C 72, 034612 (2005).

[28] L. W. Mo and Y. S. Tsai. Rev. Mod. Phys. 41, 205 (1969).

[29] P. A. M. Guichon and M. Vanderhaeghen. Phys. Rev. Lett. 91, 142303 (2003).

[30] J. Sakurai. Currents and mesons (University of Chicago Press, Chicago, 1969). 
[31] F. Iachello, A.D. Jackson, and A. Lande. Phys. Lett. B 43, 191 (1973).

[32] M. Gari and W. Krumpelmann. Phys. Rev. D 45, 1817 (1992).

[33] S. Okubo. Phys. Lett. 5, 165 (1963).

[34] E. L. Lomon. Phys. Rev. C, 64, 035204 (2001).

[35] G. Hohler, E. Pietarinen, I. Sabba Stefanescu, F. Borkowski, G. G. Simon, V. H. Walther, and R. D. Wendling. Nucl. Phys. B114, 505 (1976).

[36] G. Peter Lepage and Stanley J. Brodsky. Phys. Rev. D 22, 2157 (1980).

[37] C. W. Leemann, D. R. Douglas, and G. A. Krafft. Ann. Rev. Nucl. Part. Sci. 51, 413 (2001).

[38] W. Barry et al. Beam Position Measurement in the CEBAF Recirculating Linacs by Use of Pseudorandom Pulse Sequences, JLAB-TN-90-246 (1990).

[39] W. Barry et al. Basic Noise Considerations for CEBAF Beam Position Monitors, JLABTN-91-087 (1991).

[40] Hall A Rasters. https://hallaweb.jlab.org/wiki/index.php/Raster.

[41] D. Meekins. Hall A Target Configuration, Jefferson Lab Target Group.

[42] N. Liyanage. Technical Report, E94-010 Collabration (unpublished 2001).

[43] CODA. https://coda.jlab.org/drupal/).

[44] R. W. Michaels. https://hallaweb.jlab.org/wiki/index.php/MLU_Programming.

[45] Hall A Analyzer. https://redmine.jlab.org/projects/podd/wiki.

[46] ROOT: Data Analysis Framework. https://root.cern.ch/.

[47] B. Schmookler. Hall A 12 GeV Raster Calibration. 
[48] N. Liyanage. Optics Calibration of the Hall A High Resolution Spectrometers Using the New Optimizer, JLAB-TN-02-012 (2002).

[49] F. James and M. Winkler. Minuit2 Manual, https://root.cern.ch/.

[50] SIMC Monte Carlo. https://hallcweb.jlab.org/wiki/index.php/SIMC_Monte_Carlo.

[51] M. Berz, H. Hoffmann, and H. Wollnik. Nucl. Instrum. Meth. A 258, 402 (1987).

[52] R. C. Walker et al. Phys. Rev. D 49, 5671 (1994).

[53] R. Ent et al. Phys. Rev. C 64, 054610 (2001).

[54] Y. S. Tsai. Phys. Rev. 122, 1898 (1961).

[55] J. Schwinger. Phys. Rev. 76, 790 (1949).

[56] N. C. R. Makins. Measurement of the Nuclear Dependence and Momentum Transfer Dependence of Quasielastic (e,e'p) Scattering at Large Momentum Transfer, Ph.D. thesis, Massachusetts Institute of Technology (1994).

[57] M. Tanabashi et al. Phys. Rev. D 98, 030001 (2018).

[58] L. Ou. Precision Measurements of Electron-Proton Elastic Scattering Cross Sections at Large $Q^{2}$, Ph.D. thesis (2019).

[59] L. Ou. Correction due to external bremsstrahlung in dummy subtraction, https://hallaweb.jlab.org/dvcslog/Gmp/171.

[60] T. Gautam. LHRS acceptance study to resolve the issues with K1-1 kinematic.

[61] Y. Wang. Measurement of the Elastic Ep Cross Section at $\mathrm{Q}^{2}=0.66,1.10,1.51$ and $1.65(\mathrm{GeV})^{2}(2008)$. 


\section{VITA}

\section{THIR NARAYAN SHARMA GAUTAM}

32 Goldsmith Pl

Newport News, VA 23606 U.S.A.

Phone: 347-735-1865

email: tns.gautam@gmail.com

\section{Education}

Ph.D. (2019), Physics, Hampton University

M.Sc. (2013), Physics, The University of Southern Mississippi

\section{Oral presentations}

1. Jefferson Lab Hall A Beamline Instrumentation and Calibration for GMp experiment, 2015 Fall Meeting of the APS Division of Nuclear Physics, Oct 28 Aug 31, Santa Fe New Mexico, United States

2. Preliminary Proton Elastic Cross-Section results at High $Q^{2}$ (on behalf of GMp experiment), Hall A/C Collaboration meeting, Newport News, VA, January 18, 2017

3. Precision Measurement of the Proton Elastic Cross-Section at High $Q^{2}$, APS April Meeting 2018 Columbus, Ohio April 14-17, 2018

4. Precision Measurement of the Proton Elastic Cross-Section results at High $Q^{2}$ (on behalf of GMp experiment), Hall A/C Collaboration meeting, Newport News, VA, 22 June 2018 